\title{
Protein interactions along the presequence import pathway
}

\author{
Dissertation \\ in partial fulfilment of the requirements \\ for the degree "Doctor rerum naturalium" \\ in the Molecular Biology Program \\ at the Georg August University Göttingen, \\ Faculty of Biology
}

Submitted by

Christian Schulz

born in

Leipzig, Germany

Göttingen, September 2013 


\section{Members of the Thesis Committee}

Prof. Dr. Peter Rehling

(Supervisor and first referee)

Prof. Dr. Kai Tittmann

(Second referee)

Prof. Dr. Dirk Görlich
Institute of Cellular Biochemistry

Georg-August-University

Göttingen, Germany

Department of Bioanalytics

Georg-August-University

Göttingen, Germany

Department of Cellular Logistics

Max Planck Institute for Biophysical Chemistry

Göttingen, Germany

\section{Additional Members of the Examination Board}

Prof. Dr. Marina Rodnina

Prof. Dr. Jörg Stülke

Prof. Dr. Stefan Jakobs
Department of Physical Biochemistry

Max Planck Institute for Biophysical Chemistry

Göttingen, Germany

Department General Microbiology

Georg-August-University

Göttingen, Germany

Department of NanoBiophotonics

Max Planck Institute for Biophysical Chemistry

Göttingen, Germany

Date of oral examination: 11.11.2013 


\section{Affidavit}

I hereby declare that my dissertation, entitled 'Protein interactions along the presequence import pathway', has been written independently and with no other aids or sources than quoted.

Christian Schulz

Göttingen, September 2013 
"Success is the ability to go from one failure to another with no loss of enthusiasm."

Winston Churchill 


\section{Contents}

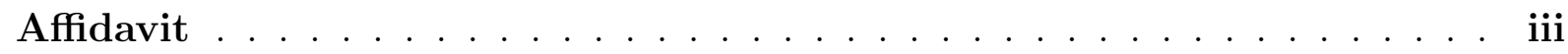

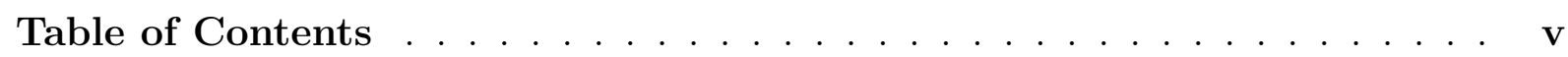

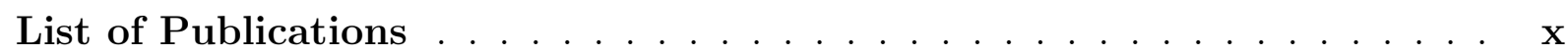

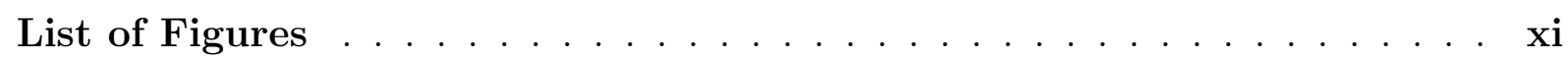

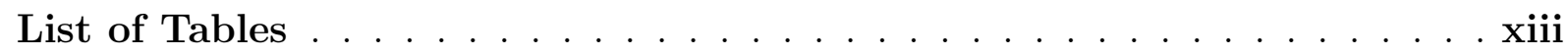

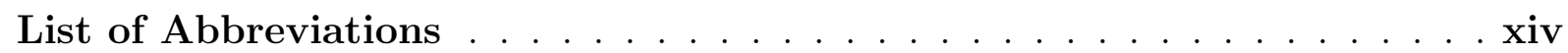

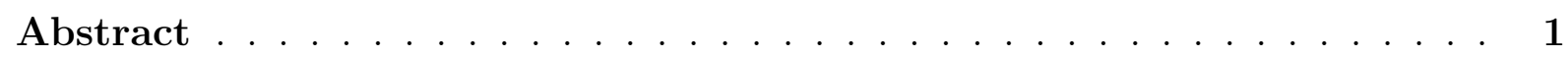

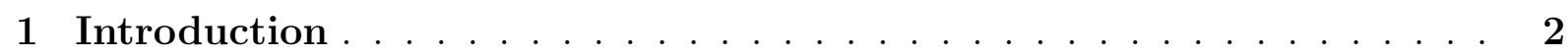

1.1 A hallmark of eukaryotic cells: organelles . . . . . . . . . . . . . 2

1.1.1 Mitochondria fulfill important roles in metabolism and signaling . . 3

1.1.2 Mitochondrial ultrastructure and dynamics . . . . . . . . . . . . 4

1.1.2.1 The endosymbiotic origin of mitochondria determines their ultrastructure . . . . . . . . . . . . . . 4 4

1.1.2.2 Mitochondria are dynamic organelles . . . . . . . . . . 5

1.2 Targeting signals and import routes into the different mitochondrial compartments .......................... 7

1.2.1 Protein transport into the outer membrane . . . . . . . . . . . 7

1.2.2 Transport of intermembrane space proteins - the MIA pathway . . . 10

1.2.3 Import of metabolite carriers - the TIM22 complex . . . . . . . . . 11

1.2.4 Protein export from the mitochondrial matrix into the inner membrane . . . . . . . . . . . . . . . . . . 12

1.2.5 Import of presequence-containing precursors - the TIM23 pathway . 13 1.2.5.1 The N-terminal mitochondrial signal peptide - the presequence . . . . . . . . . . . . . . . 13

1.2.5.2 The TOM complex mediates passage across the outer membrane ................... 14 
1.2.5.3 The TIM23 translocase drives transport across the inner membrane ................. 17

1.2.5.4 Full matrix translocation is powered by the PAM complex 23

1.3 Aim of this work . . . . . . . . . . . . . . . . 31

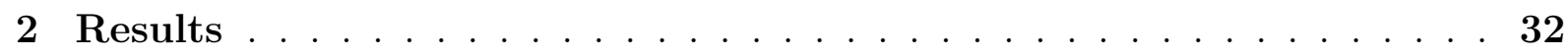

2.1 Identification of presequence binding proteins and characterization of the respective domains . . . . . . . . . . . . . . . . . . . . . . . . 32

2.1.1 Presequence photopeptides . . . . . . . . . . . . . . 32

2.1.1.1 Design of photopeptides . . . . . . . . . . . . 32

2.1.1.2 Photopeptides are specific for the presequence import path-

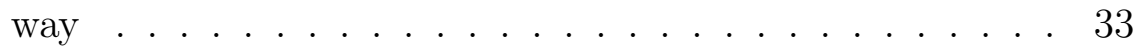

2.1.1.3 Identification of Tim50 as a novel presequence receptor . . 36

2.1.2 Identification of a presequence binding domain in Tim50 . . . . . . 38

2.1.2.1 Tim50 contains a C-terminal presequence binding domain 38

2.1.2.2 Attempts to obtain a high resolution structure of Tim50 ${ }^{\mathrm{PBD}} 42$

2.1.2.3 Tim50 contains a second presequence binding site . . . . . 42

2.1.3 Characterization of Tim50's presequence binding domain . . . . . . 46

2.1.3.1 The presequence binding domain of Tim50 is essential for cell viability . . . . . . . . . . . . . . . . 4 46

2.1.3.2 The interaction between Tim50 and Tim23 is independent of the PBD . . . . . . . . . . . . . . . . . 47

2.1.3.3 The interaction between Tim50 and presequence peptides is independent of $\operatorname{Tim} 23 \ldots$. . . . . . . . . . . . . . 49

2.1.3.4 Deletion of the PBD in Tim50 impairs presequence import across the inner membrane . . . . . . . . . . . 50

2.1.3.5 The presequence is bound to Tim50 in a complex between $\operatorname{Tim} 23$ and $\operatorname{Tim} 50$. . . . . . . . . . . . . . 54

2.2 Dynamics of TIM23 subunits during translocation of a substrate . . . . . . 56

2.2.1 Integration of subunits into the TIM23 and PAM complex . . . . . 56

2.2.2 Generation of a TOM-TIM23 supercomplex by arrest of $\mathrm{b}_{2}(167)_{\Delta^{-}}$

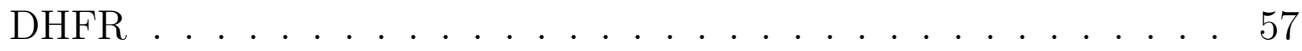

2.2.2.1 Arrest of chemical amounts of $b_{2}(167)_{\Delta}$-DHFR inhibit import ........................ 57

2.2.2.2 Isolation of the TOM-TIM23 supercomplex . . . . . . . . 59

2.2.2.3 Hsp70's ATPase activity is needed to stabilize the TOMTIM23 supercomplex . . . . . . . . . . . . . . 61

2.2.3 Integration of subunits into the active TOM-TIM23 supercomplex . 65

2.2.3.1 Tim44, Tim21, Pam18 and Tom20 integrate into the active TOM-TIM23 supercomplex . . . . . . . . . . 65

2.2.4 Different TIM23 or PAM mutants display contradicting phenotypes 69 
2.2.4.1 TIM23 complex composition in mutants . . . . . . . . 69

2.2.4.2 Import and inward driving activity in mutants . . . . . . . 70

2.2.4.3 Efficient assembly of Pam18, but not Tim44, depends on Mgr2 . . . . . . . . . . . . . . 72

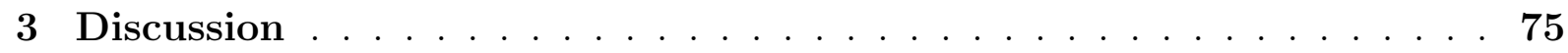

3.1 Presequence photopeptides as a tool to identify presequence receptors . . . 75

3.2 Tim50 - an essential presequence receptor of the TIM23 complex . . . . . 80

3.2.1 Tim50 contains an essential C-terminal presequence binding domain 80

3.2.2 Presequence recognition by Tim50s PBD is needed for transport across the inner membrane . . . . . . . . . . . . . . . 82

3.2.3 Handover is coordinated in a trimeric complex between the presequence, Tim23 and Tim50 . . . . . . . . . . . . . . . 84

3.2.4 Model of early transport steps by the TIM23 translocase . . . . . . 85

3.3 Subunit oscillation in the active translocase . . . . . . . . . . . 87

3.3.1 In vitro import and assembly of TIM23 and PAM subunits . . . . . 87

3.3.2 The TOM-TIM23 supercomplex depends on the import motor . . . 88

3.3.3 An experimental setup to assay subunit integration into the active translocase . . . . . . . . . . . . . . . . . . . . . 89

3.3.4 Tim21, Tim44, and Pam18 oscillate in the active PAM complex . . 91

3.3.5 The structure and function of TIM23 and PAM in mutant mitochondria . . . . . . . . . . . . . . . . . 92

3.3.6 Mgr2 is involved in the oscillation of Pam18 . . . . . . . . . . . . 94

3.3.7 Model of matrix translocation by the TIM23 complex . . . . . . . . 95

4 Summary and Conclusion . . . . . . . . . . . . . . 97

5 Materials and Methods . . . . . . . . . . . . . . . . . 99

5.1 Materials . . . . . . . . . . . . . . . . . . . . . . . . 99

5.1 .1 Kit systems and reagents . . . . . . . . . . . . . . . . 99

5.1 .2 Microorganisms . . . . . . . . . . . . . . . . . 102

5.1 .3 Plasmids . . . . . . . . . . . . . . . . . . . . . 107

5.1 .4 Peptides . . . . . . . . . . . . . . . . . . . . . . 113

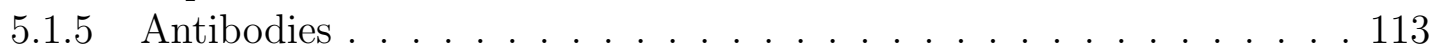

5.1 .6 Instruments and software . . . . . . . . . . . . . . . 114

5.2 Methods . . . . . . . . . . . . . . . . . . 115

5.2.1 Handling of biological material . . . . . . . . . . . . . . 115

5.2.1.1 E. coli growth . . . . . . . . . . . . . . . . 115

5.2.1.2 E. coli transformation . . . . . . . . . . . . . 115

5.2 .1 .3 Yeast growth . . . . . . . . . . . . . . . . . 116

5.2.1.4 Transformation of yeast . . . . . . . . . . . 116 
5.2.1.5 Sporulation and dissection of tetrads . . . . . . . . . 117

5.2.1.6 Whole cell lysate of yeast . . . . . . . . . . . . . 117

5.2.1.7 Preparation of S. cerevisiae mitochondria . . . . . . . . 118

5.2 .2 Molecular biology . . . . . . . . . . . . . . . . . . . . . . . . . . 119

5.2.2.1 Isolation of yeast genomic DNA . . . . . . . . . . . 119

5.2.2.2 Isolation of plasmid DNA from E. coli . . . . . . . . . . 119

5.2.2.3 PCR . . . . . . . . . . . . . . . . . . . . . 119

5.2.2.4 Cloning . . . . . . . . . . . . . . . . . 120

5.2 .2 .5 In vitro mutagenesis . . . . . . . . . . . . . . . . . 120

5.2.2.6 In vitro transcription . . . . . . . . . . . . . . . . . . . . 121

5.2.2.7 In vitro translation . . . . . . . . . . . . . . . . . . . . . . 121

5.2.3 Protein biochemistry . . . . . . . . . . . . . . . . . . 123

5.2 .3 .1 SDS-PAGE . . . . . . . . . . . . . . . . 123

5.2 .3 .2 BN-PAGE . . . . . . . . . . . . . . . . . 123

5.2.3.3 Ponceau S staining of PVDF membranes . . . . . . . . . . 124

5.2.3.4 Coomassie staining of gels and PVDF membranes . . . . . 124

5.2.3.5 Autoradiography . . . . . . . . . . . . . . . . . 125

5.2.3.6 Western blot . . . . . . . . . . . . . . . 125

5.2.3.7 Immunodetection . . . . . . . . . . . . . . . . . . 125

5.2.3.8 Protein purification . . . . . . . . . . . . . 126

5.2.3.9 Affinity purification of antisera . . . . . . . . . . . . 129

5.2.4 Specialized assays . . . . . . . . . . . . . . . . . 129

5.2.4.1 Protein import in mitochondria . . . . . . . . . . . . 129

5.2 .4 .2 Inward driving activity . . . . . . . . . . . . . . 130

5.2.4.3 Generation of transport intermediate arrested in the TOM complex . . . . . . . . . . . . . . 130

5.2.4.4 Generation of transport intermediate arrested in the TOMTIM23 supercomplex . . . . . . . . . . . . . . 131

5.2.4.5 Photo-Crosslinking . . . . . . . . . . . . . . 131

5.2.4.6 Chemical Crosslinking . . . . . . . . . . . . . . . 132

5.2.4.7 Surface plasmon resonance . . . . . . . . . . . . . . 133

5.2.4.8 Mass spectrometic analysis of photoadducts . . . . . . . 133

5.2.4.9 HA co-immunoprecipitations . . . . . . . . . . . . . . 134

5.2.4.10 Immobilization of antibodies on protein A sepharose . . . 135

5.2.4.11 $\alpha$-Tim23 co-immunoprecipitation . . . . . . . . . . 135

5.2.4.12 Induction of temperature-sensitive phenotype . . . . . . . 136

5.2.4.13 Preparation of IgG sepharose . . . . . . . . . . . . . . 136

$5.2 .4 .14 \operatorname{Tim}_{2} 3^{\mathrm{PA}}$ isolation . . . . . . . . . . . . . . . . 136

5.2.4.15 Isolation of TOM-TIM23 supercomplex . . . . . . . . . 137

5.2.4.16 Tim23 pulldown . . . . . . . . . . . . . . . . 137

5.2.4.17 Measurment of membrane potential . . . . . . . . . 138 


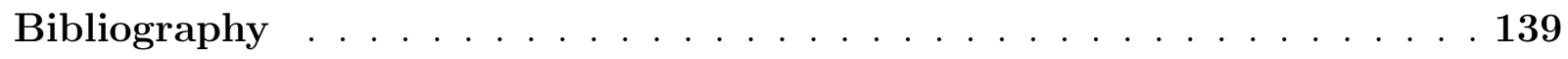

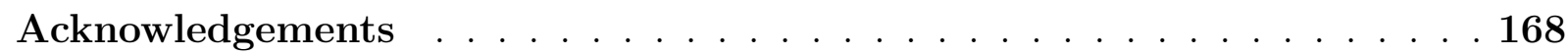




\section{List of Publications}

Parts of this thesis have been published in the following articles:

Schulz, C., Lytovchenko, O., Melin, J., Chacinska, A., Guiard, B., et al. (2011). Tim50's presequence receptor domain is essential for signal driven transport across the TIM23 complex. J Cell Biol, 195(4), 643-656.

Reinhold, R., Krüger, V., Meinecke, M., Schulz, C., Schmidt, B., et al. (2012). The Channel-Forming Sym1 Protein Is Transported by the TIM23 Complex in a Presequence-Independent Manner. Mol Cell Biol, 32(24), 5009-5021.*

Lytovchenko, O., Melin, J., Schulz, C., Kilisch, M., Hutu, D. P. and Rehling, P. (2013). Signal recognition initiates reorganization of the presequence translocase during protein import. EMBO J, 32, 886-898.

Parts of this thesis will be communicated in the following publication:

Schulz, C. and Rehling, P. A module swap mechanism drives precursor transport through the mitochondrial presequence translocase. Manuscript in preparation.

${ }^{*}$ results of this publication are not discussed here 


\section{List of Figures}

Fig. 1 Compartmentalization of a simplified eukaryotic cell. . . . . . . . . . . 3

Fig. 2 Mitochondrial ultrastructure . . . . . . . . . . . . . 6

Fig. 3 Mitochondrial protein import pathways . . . . . . . . . . . 8

Fig. 4 The TOM complex . . . . . . . . . . . . . . 16

Fig. 5 The TIM23 and PAM complex . . . . . . . . . . . . . . . 20

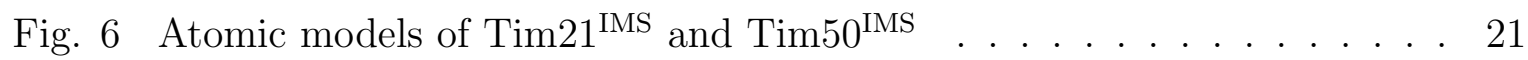

Fig. 7 Atomic model of the Pam16-Pam18 complex . . . . . . . . . . . . . 28

Fig. 8 The Hsp70 cycle during protein import . . . . . . . . . . . . . . . . 30

Fig. 9 Design of presequence photopeptides . . . . . . . . . . . . . . . . 33

Fig. 10 Photopeptides import into mitochondria . . . . . . . . . . . . . . . 34

Fig. 11 Photopeptides inhibit presequence import . . . . . . . . . . . . . . 35

Fig. 12 Import inhibition by presequence peptides is reversible . . . . . . . . . 36

Fig. 13 Photopeptides crosslink to presequence receptors in organello . . . . . . 37

Fig. 14 Isolation of photoadducts to presequence receptors . . . . . . . . . . . . 38

Fig. 15 Tim50 domains and identification of presequence binding domain . . . . 40

Fig. 16 Deletion of the presequence binding domain abolishes presequence crosslink-

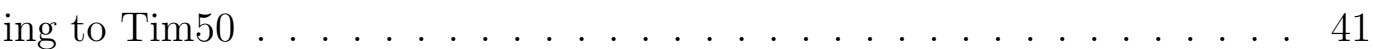

Fig. 17 Tim50 ${ }^{\mathrm{PBD}}$ photoadducts contain one peptide in different configurations . 43

Fig. 18 Purification of Tim50 ${ }^{\mathrm{PBD}}$ and crystallization trials . . . . . . . . . . 44

Fig. $19 \operatorname{Tim}^{\mathrm{C}}{ }^{\mathrm{C}}$ crosslinks more efficiently to presequences than Tim50 ${ }^{\triangle \mathrm{PBD}}$. . 45

Fig. 20 Tim50 $0^{\mathrm{PBD}}$ is essential for cell viability . . . . . . . . . . . . . . 48

Fig. 21 The Tim50 presequence binding domain and Tim23 binding domain are separate. . . . . . . . . . . . . . . 5 50

Fig. 22 Presequence binding to Tim50 is independent of Tim23 . . . . . . . . . 51

Fig. 23 Deletion of the Tim50 ${ }^{\mathrm{PBD}}$ affects presequence import. . . . . . . . . 53 
Fig. $24 \operatorname{Tim} 50 \downarrow+\operatorname{Tim} 50^{\mathrm{HA}}$ and Tim50 $\downarrow+\operatorname{Tim} 50^{\Delta \mathrm{PBD}-\mathrm{HA}}$ have a similar membrane potential . . . . . . . . . . . . . . . . . 5 54

Fig. 25 A trimeric complex between Tim23, Tim50 and the presequence . . . . 55

Fig. 26 Co-isolation of TIM23 and PAM subunits with Tim23 $3^{\mathrm{PA}} \ldots \ldots$. . . . 58

Fig. 27 Chemical amounts of $b_{2}(167)_{\Delta}$-DHFR block presequence import. . . . . 60

Fig. 28 Isolation and separation of the TOM-TIM23 supercomplex. . . . . . . . 61

Fig. 29 Generation of the TOM-TIM23 supercomplex depends on the import efficiency . . . . . . . . . . . . . . . . . 62

Fig. 30 Stability of the TOM-TIM23 supercomplex depends on the Hsp70 activity 63

Fig. 31 TIM23 can be specifically isolated by $\alpha$-Tim23 co-immunoprecipitations 64

Fig. 32 Temperature-sensitive mutants of PAM components reveal reduced association with the TIM23 complex . . . . . . . . . . . 66

Fig. 33 Regulatory subunits integrate into the active TIM23 translocase . . . . 68

Fig. 34 Deletion mutants of TIM23 and PAM components reveal a normal composition of the TIM23 complex . . . . . . . . . . . . . . . 70

Fig. 35 TIM23 and PAM mutants display differential import phenotypes . . . . 72

Fig. 36 TIM23 and PAM mutants display differential inward driving activity . . 73

Fig. 37 Integration of Pam18 into TIM23 is impaired in mgr2 $\Delta . . . . . .74$

Fig. 38 Distances between Tom20 and pALDH . . . . . . . . . . . . . 78

Fig. 39 Comparison between S.c. and H.s. Tim50 . . . . . . . . . . . . . . . . . 83

Fig. 40 Presequence recognition and precursor transport in the IMS . . . . . . . 85

Fig. 41 Model of subunit oscillation during import motor function . . . . . . . . 96 


\section{List of Tables}

Tab. 1 The TOM complex ....................... 15

Tab. 2 The TIM23 complex .................... 20

Tab. 3 The PAM complex ........................ 29

Tab. 4 Affinities of different Tim50 domains to presequence peptides . . . . . . 46

Tab. 5 Commercial kits . . . . . . . . . . . . . . . . . . . . 99

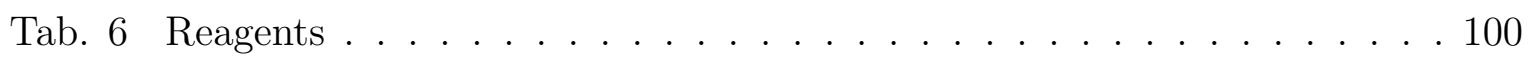

Tab. 7 Yeast strains . . . . . . . . . . . . . . . . . . . . . 103

Tab. 8 E. coli strains . . . . . . . . . . . . . . . . 107

Tab. 9 Plasmids . . . . . . . . . . . . . . . . . . . . . 108

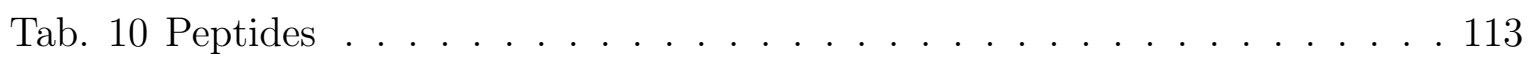

Tab. 11 Instruments . . . . . . . . . . . . . . . . . . . . 114

Tab. 12 Software . . . . . . . . . . . . . . . . . 115

Tab. 13 Conditions for radiolabeling of different precursors. . . . . . . . . . . . 122

Tab. 14 Protein expression and purification conditions . . . . . . . . . . 128 


\section{List of Abbreviations}

$\downarrow \quad$ down regulation

$\uparrow \quad$ up regulation

$\Delta \psi \quad$ Membrane potential across the inner membrane

AAC $\quad \mathrm{ADP} / \mathrm{ATP}$ carrier

AEBSF 4-(2-Aminoethyl) benzenesulfonyl fluoride hydrochloride

APS Ammonium persulfate

ATP Adenosine triphosphate

AVO Antimycin A, valinomycin, oligomycin mixture

BPA Para-benzoylphenylalanine

BSA Bovine serum albumin

CJ Cristae junction

CK Creatine kinase

co-IP Co-immunoprecipitation

CP Creatine phosphate

DEPC Diethylpyrocarbonate

DFDNB 1,5-difluoro-2,4-dinitrobenzene

$\mathrm{dH}_{2} \mathrm{O} \quad$ Deionized water

DHFR Mouse dihydrofolate reductase

DMP Dimethy pimelimidate dihydrochloride

DMSO Dimethyl sulfoxide

E Eluate

ECL Enhanced chemiluminescence

EDTA Ethylenediaminetetraacetic acid

ER Endoplasmic reticulum

HEPES 4-(2-hydroxyethyl)-1-piperazineethanesulfonic acid

HRP Horse radish peroxidase

IBM Inner boundary membrane 
IM Inner mitochondrial membrane

IMAC Immobilized-metal affinity chromatography

IMP Intermediate processing peptidase

IMS Intermembrane space

LB Lysogeny broth

MALDI Matrix-assisted laser desorption/ionization

MIA Mitochondrial IMS assembly machinery

MIM Mitochondrial import complex

MOPS 3-(N-morpholino)propanesulfonic acid

MPP Mitochondrial processing peptidase

MS Mass spectrometry

mtDNA Mitochondria DNA

MTX Methotrexate

NADH Nicotinamide adenine dinucleotide

NADPH Nicotinamide adenine dinucleotide phosphate

NAT Nourseothricin

OM Outer mitochondrial membrane

ORF Open reading frame

OXA Export and assembly machinery of the inner membrane

PA Photoadduct

PA Protein A

pALDH Aldehyde dehydrogenase presequence

PAM Presequence translocase-associated motor

PBD Presequence binding domain

PBS Phosphate buffered saline

pCox4 Presequence of subunit IV of cytochrome $c$ oxidase

PDB Protein database

PK Proteinase K

PMSF Phenylmethylsulfonyl fluoride

PVDF Polyvinylidene fluoride

SA-HRP Streptavidin - horse radish peroxidase 
SAM Sorting and assembly machinery

SPR Surface plasmon resonance

Tat Twin arginine translocation

TBS Tris buffered saline

TCA Trichloroacetic acid

TEMED N,N,N',N'-tetramethylethylenediamine

TFA Trifluoroacetic acid

TIM22 Carrier translocase of the inner membrane

TIM23 Presequence translocase of the inner membrane

TMD Transmembrane domain

TO Total

TOM Translocase of the outer membrane

ts temperature sensitive

U Unbound

UV Ultra violet

YPAD YPD with adenine

YPD Yeast extract, peptone, glucose

YPG Yeast extract, peptone, glycerol

YPL Yeast extract, peptone, lactate 


\section{Abstract}

In this thesis the mechanisms of protein transport by the translocase of the inner mitochondrial membrane (TIM23) was investigated. Purified presequence peptides containing a p-benzophenyalalanine were established as a tool to identify and proximate presequence binding sites. Receptors could be identified, revealing Tim50 as a so far unknown presequence receptor of TIM23. It contains two separate binding sites in its intermembrane space (IMS) domain. One is formed by the C-terminal presequence binding domain (PBD) and the second by a negatively charged groove located in the core domain.

The PBD is needed for efficient transport across the inner mitochondrial membrane, rendering it essential for cell viability in yeast. It is not involved in the establishment of transport intermediates at the level of the outer membrane translocase (TOM), recruitment of Tim50 to TIM23 and the regulation of Tim50s interaction with Tim21. The presequence hand-over in the IMS occurs from Tim $50^{\mathrm{IMS}}$ to Tim $23^{\mathrm{IMS}}$. In this process a trimeric complex is formed, with Tim50 binding the presequence as well as Tim23. Subsequently, Tim23 receives the presequence and dissociates from Tim50 due to overlapping binding sites.

Additionally, an assay to test integration of subunits into the active TIM23 complex was established. It made use of the two membrane spanning translocation intermediate generated by arresting $b_{2}(167)_{\Delta}$-DHFR. The formed supercomplex required the ATPase activity of Hsp70 in the import motor (PAM). Using this assay it was shown that the PAM subunits Tim44 and Pam18 as well as the TIM23 subunit Tim21 integrated into the active TIM23 complex.

The oscillation between free and translocase-bound Pam18 depends on Mgr2, but not Tim21. In contrast Tim44 oscillated Mgr2 independent. Hence, the regulatory subunits of the import motor seem to follow the cyclic recruitment of Hsp70 which is recruited to the translocase exit site by Tim44 in the ATP state in order to engage the precursor and diffuses into the matrix upon Pam18 stimulated ATP hydrolysis. Conformational changes within Tim44 and Pam18 during this activation process might lead to their loss from the translocase and therefore requires continuous recruitment to the active TIM23 complex. 


\section{Introduction}

\subsection{A hallmark of eukaryotic cells: organelles}

The most evident visual difference between prokaryotic and eukaryotic cells is the larger size and the compartmentalization of the eukaryotic cell (Palade, 1964) (Figure 1). All organelles are enclosed by at least one membrane, which can be traced back to the endocytosed plasma membrane that established these organelles (de Duve, 2007). Biological membranes provide a hydrophobic barrier that is used to separate molecules and generate gradients, e.g. the proton gradient (proton motive force) utilized for ATP synthesis. Additionally, membranes are the place of lipid synthesis and remodeling. Because of their importance for cellular processes the available surface of biological membranes is a limiting factor for the cell.

In the eukaryotic cell the membrane surface is increased by establishing intracellular organelles. This provides the additional advantage to separate reactions in order to increase their efficiency. Most of the energy production is for example located at the inner mitochondrial membrane, while energy consumption is spread throughout the entire cell. Furthermore, damaging agents can be enclosed in order to protect other cellular components (proteases in the lysosome/ vacuole). Organelles are also used for storage, e.g. $\mathrm{Ca}^{2+}$ in the ER.

Except for the nucleus, mitochondria and chloroplasts none of the other organelles contain genetic information. Most of the genetic information of the latter two was transferred to the nuclear genome during evolution (Daley et al., 2002). This imposed a new challenge for the eukaryotic cell: in order to use these organelles, proteins and metabolites need to be transported across the organellar membranes. This is achieved by dedicated protein translocases and metabolite carriers in the respective membranes. These principles evolved already in prokaryotes where the SecYEG is used to secret soluble or insert proteins into the plasma membrane (Park and Rapoport, 2012).

In eukaryotes, with their plethora of different organelles, several different mechanisms of protein targeting and transport evolved. In this study the mechanism of protein translo- 


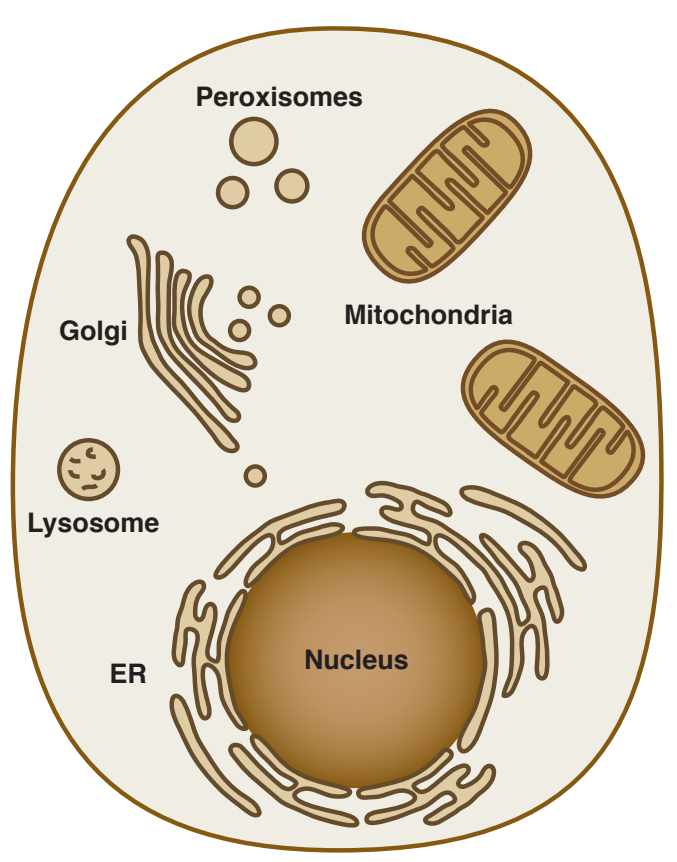

Fig. 1: Compartmentalization of a simple eukaryotic cell. The plasma membrane encloses the cell. Within the cytosol several membrane-enclosed compartments are present. The nucleus, the endoplasmic reticulum (ER), the Golgi apparatus, peroxisomes, lysosomes (vacuole) and mitochondria. Plant cells contain chloroplasts in addition (not displayed).

cation of presequence targeted precursor proteins into mitochondria will be discussed. Since most studies on mitochondrial protein transport were performed in S. cerevisiae, the following sections will focus on the process in this species (Maccecchini et al., 1979; Neupert and Schatz, 1981; Pfanner et al., 1996).

\subsubsection{Mitochondria fulfill important roles in metabolism and signaling}

Mitochondria are widely recognized by their central role in the energy metabolism and the ATP generation by the respiratory chain (Saraste, 1999). It is this separation of the energy production from the remainder of the cell that boosted genome complexity in eukaryotic cells (Lane and Martin, 2010).

Moreover, mitochondria are involved in a diverse set of other important cellular functions. Essential steps of the iron sulfur cluster biogenesis are carried out in mitochondria, 
making the organelle itself essential, even in organisms that do not depend on respiratory growth (van der Giezen and Tovar, 2005; Shiflett and Johnson, 2010; Lill et al., 2012). Several other metabolic pathways are, at least partially, located in mitochondria: for example the TCA cycle, urea cycle and amino acid metabolism.

Furthermore, these organelles are important in the regulation of cellular processes. Their role in apoptosis is well established (Green and Reed, 1998) and the contribution to calcium signaling and buffering was recently revealed (Clapham, 2007; Campello and Scorrano, 2010).

In lipid metabolism the close contact between the ER and mitochondria (via the ERMES complex) is important for lipid exchange (Nguyen et al., 2012; Rowland and Voeltz, 2012; Voss et al., 2012).

\subsubsection{Mitochondrial ultrastructure and dynamics}

\subsubsection{The endosymbiotic origin of mitochondria determines their ultra- structure}

Mitochondria developed by the endosymbiosis of an $\alpha$-proteobacterium by a so far unidentified host (Andersson et al., 1998; Szklarczyk and Huynen, 2010; Lithgow and Schneider, 2010). Consequently, the inner and outer mitochondrial membranes correspond to the plasma membranes of the endosymbiont and the host, respectively. The evolution of the protein translocase machineries was necessary to allow the transfer of genetic material to the nucleus. Whether the establishment of the translocases was imposed by the host or the endosymbiont or a mixture of both is debated (Alcock et al., 2010). In yeast about $99 \%$ of all mitochondrial proteins ( 1000) need to be imported into mitochondria after their translation by cytosolic ribosomes (Sickmann et al., 2003).

Despite the transfer of genetic material, a few proteins are still encoded by the mtDNA (eight in S. cerevisiae). The majority of these are the highly hydrophobic core proteins of the respiratory chain complexes (Wallace, 2007). A complete replication, transcription, translation and export machinery (making up as much as $\sim 25 \%$ of the mitochondrial proteome) is maintained in order to ensure proper expression of these proteins (Sickmann et al., 2003; Smits et al., 2010).

The two mitochondrial membranes enclose an aqueous compartment, the intermembrane space (Figure 2). The outer mitochondrial membrane (OM) contains the $\beta$-barrel 
protein porin and hence does not provide a significant diffusion barrier to ions and metabolites (Benz, 1994). In contrast, the inner mitochondrial membrane (IM) is tightly sealed in order to maintain the proton gradient that is generated by the respiratory chain and harvested by the ATP synthase.

The inner membrane is composed of different domains: the inner boundary membrane, which is opposed to the outer membrane, the crista junction where the inner boundary membrane turns inward to form the cristae, long invaginations into the mitochondrial matrix. The crista junctions are stabilized by Fcj1 (formation of crista junctions 1) and other MINOS complex subunits (mitochondrial inner membrane organizing system) (van der Laan et al., 2012). The tips of the cristae are formed and stabilized by dimers of the $\mathrm{F}_{0} \mathrm{~F}_{1}$ ATP synthase (Paumard et al., 2002).

Different protein complexes of the inner membrane distribute in these different domains according to their function: the respiratory chain complexes are mainly found in the cristae membrane, just like the insertase for the mitochondrially encoded proteins OXA (Vogel et al., 2006). Therefore, the intermembrane space enclosed in the cristae traps protons leading to a high local concentration used by the ATP synthase, which is located at the high curvature regions of the cristae (Strauss et al., 2008). Protein import components have a preference for the inner boundary membrane where they are in close contact to the translocase of the outer membrane (TOM complex) (Vogel et al., 2006). Disruption of the mitochondrial ultrastructure impairs function of these protein complexes (Neupert, 2012).

\subsubsection{Mitochondria are dynamic organelles}

In contrast to the common depiction as short rods, mitochondria exist as an interconnected network (Messerschmitt et al., 2003; Von Der Malsburg et al., 2011). The network undergoes constant fusion, fission and removal of damaged parts (mitophagy). Fusion and fission allow the cell to adapt the mitochondrial network to the changing environmental demands. Fission generates small fragmented mitochondria, while fusion increases the connectivity of the network (Westermann, 2010). The large network seems to be beneficial for the metabolism as it supports energy distribution. The balanced interplay of both processes is important for the maintenance of mitochondrial functions.

Apart from this, the mitochondrial network is constantly changed by the removal of damaged mitochondria, mitophagy (Youle and Narendra, 2011). Damaged organelles are 


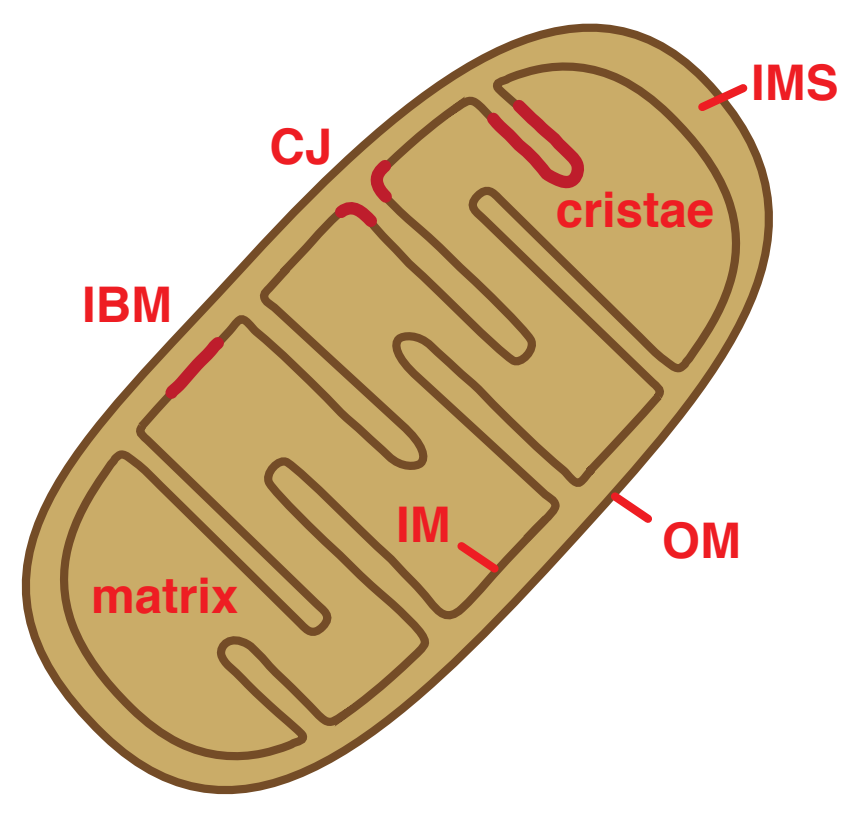

Fig. 2: Mitochondrial ultrastructure - The outer membrane (OM) encloses the inter membrane space (IMS) and the inner membrane (IM). The inner membrane contains several domains (red): the inner boundary membrane (IBM), the cristae junctions (CJ) and the cristae. It encloses the mitochondrial matrix.

selectively recognized, sequestered into the autophagosome and subsequently degraded in the lysosome. The mechanism of marking damaged mitochondria and the subsequent recognition of the mark is not very well understood yet and is a focus of current research. In addition to the quality control on the level of the complete organelle, single proteins are subject to control mechanisms, either inside the organelle by means of chaperones and proteases (Voos, 2013) or on its surface using the ubiquitin/proteasome system (Heo et al., 2010).

In summary, the constant dynamic changes that mitochondria undergo require continuous biogenesis, which largely relies on import of nuclear encoded proteins. 


\subsection{Targeting signals and import routes into the dif- ferent mitochondrial compartments}

The transfer of most mitochondrial genes to the nuclear genome imposed the problem that the proteins are now translated by cytosolic ribosomes and have to be imported in order to fulfill their functions in the organelle. Several decades ago the signal-hypothesis was formulated (Blobel and Dobberstein, 1975) and later shown to be true not only for the ER but also for mitochondrial protein import (Neupert and Schatz, 1981). The signalhypothesis stated that precursor proteins destined for an organelle compartment carry a signal peptide determining the transport destination. This signal is located at the Nterminus of the protein and is cleaved after import (Blobel and Dobberstein, 1975). The basic principle of a signal-directed transport holds still true for mitochondrial translocation, however the nature of the signals is now known to be much more diverse (Chacinska et al., 2009).

The initial delivery of the precursors to mitochondria seems to be post translational and partially assisted by chaperones (Neupert and Schatz, 1981; Komiya et al., 1996). In vitro, purified precursor proteins can be imported into the mitochondrial matrix without any additional cytosolic factors (Eilers and Schatz, 1986). The import process in vivo is enhanced for some precursor proteins by targeting of their mRNAs to the mitochondrial surface using a signal in the 3'-UTR (Marc et al., 2002; Sylvestre et al., 2003; Eliyahu et al., 2009; Weis et al., 2013). Furthermore, co-translational transport has been observed and might be necessary for some precursors (Verner, 1993).

The different targeting signals and transport routes into the different mitochondrial subcompartments will be introduced in the following sections (Figure 3).

\subsubsection{Protein transport into the outer membrane}

The hallmark protein type of the outer mitochondrial membrane are $\beta$-barrel proteins, e.g. Por1, Tom40 or Sam50 (Zahedi et al., 2006). Sorting of these proteins is achieved by the $\beta$-signal (Kutik et al., 2008). It is comprised of the most C-terminal $\beta$-strand with a polar-X-Gly-XX-hydrophobic-hydrophobic-X motif.

Transport of $\beta$-barrel proteins starts with the recognition of the precursor by receptors of the TOM complex (Figure 3). The nature of the signal as well as the receptor is still 


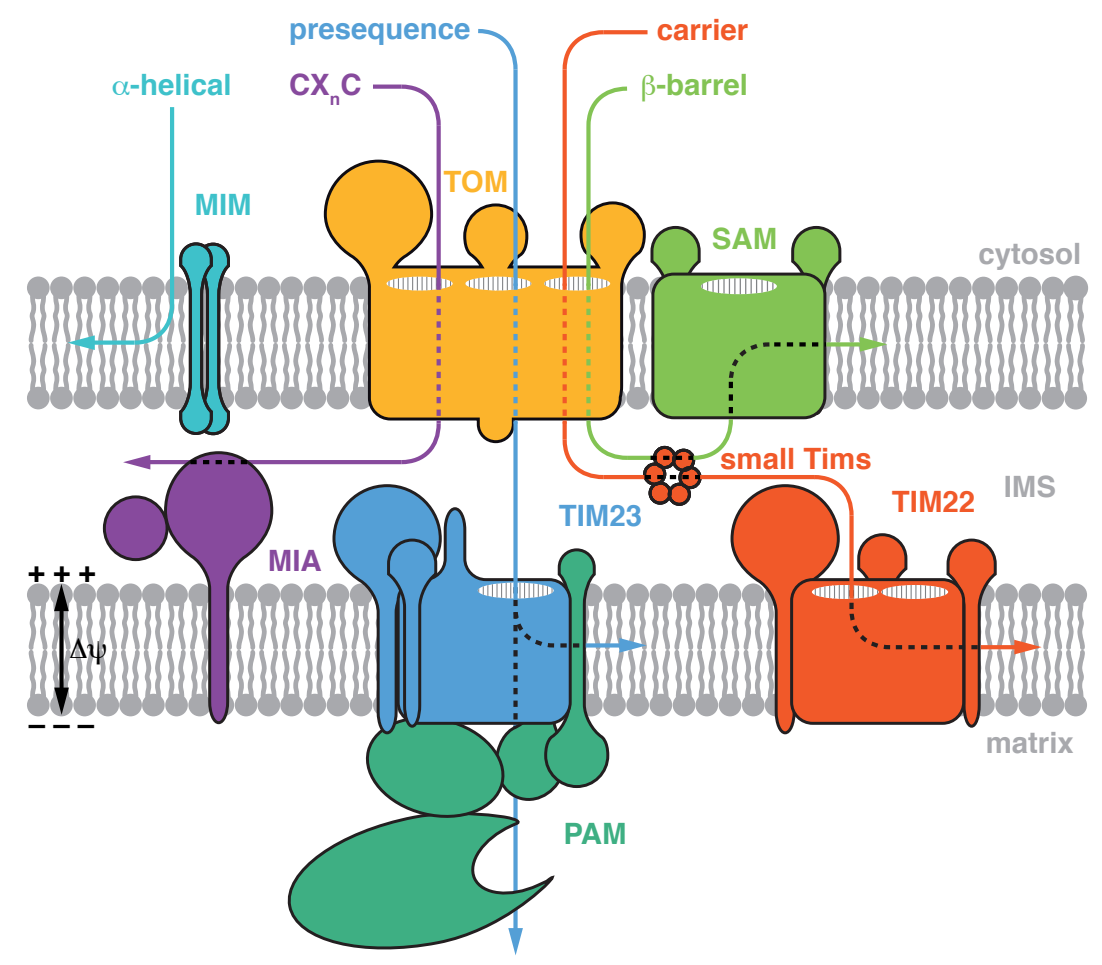

Fig. 3: Schematic representation of the mitochondrial protein import pathways The outer and inner membrane separate the cytosol, the intermembrane space (IMS) and the matrix. The membrane potential across the inner membrane $(\Delta \psi)$ is generated by the respiratory chain (not depicted). $\alpha$-helical proteins are transported in a TOM independent manner using the MIM complex. All other precursor classes use the TOM complex as a general entry gate. $\mathrm{CX}_{\mathrm{n}} \mathrm{C}$ motif containing precursors are translocated by the MIA complex into the IMS. The small Tim proteins as well as the SAM complex facilitate $\beta$-barrel protein insertion into the outer membrane. The SAM and TOM complex form a supercomplex. Additionally, the small Tim proteins participate in the import of carrier proteins by the TIM22 translocase. The TIM23 complex facilitates import into the inner membrane. Full matrix translocation via the TIM23 requires the import motor (PAM). 
unclear, however a TOM signal N-terminal to the $\beta$-signal was discussed (Chacinska et al., 2009; Dudek et al., 2013). Subsequently, the precursor is translocated through the channel formed by Tom40 (Hill et al., 1998; Krimmer et al., 2001). On the intermembrane space side the precursor is engaged by the small Tim proteins, namely the Tim9-Tim10 complex (Wiedemann et al., 2004). These ring-like chaperone complexes are formed by alternating three copies of each protein (hexamer) (Webb et al., 2006). Subsequently, the $\beta$-signal targets the precursor to the sorting and assembly machinery (SAM complex) (Kutik et al., 2008). In fact the TOM and SAM complexes engage to form a supercomplex to facilitate precursor insertion into the outer membrane. This interaction between both translocases is mediated by the central TOM receptor subunit Tom22 and the central SAM subunit Sam50 (Qiu et al., 2013).

Sam50 is the $\beta$-barrel channel of the SAM complex. Additional subunits include Sam35 and Sam37. although the precise mechanism is still unclear, it is speculated that the $\beta$ barrel precursor is fed into the aqueous space between several Sam50 subunits, where it folds in a protected environment (Walther and Rapaport, 2009). Lateral release from the inside of a single Sam50 $\beta$-barrel seems unlikely due to its rigid structure (Haltia and Freire, 1995).

The outer membrane also contains proteins with a classical $\alpha$-helical transmembrane segment. The targeting signal co-localizes with the transmembrane domain (TMD) and is referred to as signal anchor sequence in the case of a N-terminal TMD (e.g. Tom20 and Tom70), internal signal for internal TMD (e.g. Tom22 and Atg32) and tail-anchor for C-terminal TMDs (Tom5 and Tom6) (Chacinska et al., 2009). The signal anchor includes the moderately hydrophobic TMD as well as a net positively charged C-terminal flanking region, however there is no strict motif in the signal (Kanaji et al., 2000; Rapaport, 2003). Similarly, the tail-anchor is not defined as a strict motif, but rather as moderately hydrophobic rather short C-terminal TMD that is flanked by positively charges residues (Beilharz et al., 2003; Rapaport, 2003).

Import of signal-anchored outer membrane proteins does neither depend on the channel of the TOM complex, nor its receptors (Schneider et al., 1991; Meineke et al., 2008). Instead the MIM complex (mitochondrial import complex) facilitates membrane insertion of these substrates (Figure 3) (Becker et al., 2008; Dimmer et al., 2012). Furthermore, MIM assists polytopic outer membrane proteins during insertion into the lipid bilayer (e.g. Ugo1) (Becker et al., 2011; Papic et al., 2011; Dimmer et al., 2012). The MIM 
complex has a size of $\sim 200 \mathrm{kDa}$ as observed on BN-PAGE (Popov-Celeketić et al., 2008b) and contains at least Mim1 and Mim2 (Becker et al., 2008; Dimmer et al., 2012).

In contrast to all other import pathways described here, insertion of tail-anchored proteins into the outer membrane seems to work independently of a proteinaceous machinery, instead the levels of ergosterol seem to be an important determinant of the targeting specificity (Kemper et al., 2008; Krumpe et al., 2012).

\subsubsection{Transport of intermembrane space proteins - the MIA pathway}

Soluble proteins of the intermembrane space (IMS) can follow the mitochondrial intermembrane space import and assembly (MIA) pathway (Vögtle et al., 2012). The mitochondrial IMS-sorting signal (MISS) of this pathway includes an aromatic-XX-hydrophobichydrophobic-XXC signal that mediates binding to the oxidoreductase Mia40 (Figure 3) (Sideris et al., 2009). It serves to position mechanistically important cysteine residues for oxidation. These cysteines are arranged as a twin $\mathrm{CX}_{9} \mathrm{C}$ (e.g. Cox17 and Mia40) or twin $\mathrm{CX}_{3} \mathrm{C}$ (e.g. Tim9 and 10) motif (Herrmann and Riemer, 2012). After import these proteins usually form a helix-loop-helix that is stabilized by disulfide bonds of the $\mathrm{CX}_{n} \mathrm{C}$ motif.

Import of $\mathrm{CX}_{3} \mathrm{C}$ or $\mathrm{CX}_{9} \mathrm{C}$ precursors into the intermembrane space depends on a disulfide relay system. Initial recognition and transport through the TOM complex are not yet understood. After the precursor emerges from the TOM complex it is engaged by the oxidoreductase Mia40. This is facilitated by the close vicinity of Mia40 to the IMS side of the TOM complex which is mediated by Fcj1 (Von Der Malsburg et al., 2011). Mia40 engages the MISS signal of the precursor and forms intermolecular disulfide bonds (Sideris et al., 2009). Therefore, the precursor is trapped in the intermembrane space and folding is assisted as the disulfide bonds are rearranged to intramolecular bridges. In order to operate in a subsequent round of import Mia40 is re-oxidized by Erv1, which shuttles the electrons to the cytochrome $c$ oxidase (Bihlmaier et al., 2007; Stojanovski et al., 2008). 


\subsubsection{Import of metabolite carriers - the TIM22 complex}

Metabolite carrier proteins are six transmembrane domain containing inner membrane proteins (e.g. ADP-ATP carrier (AAC) and phosphate carrier (PiC)). Their targeting signals are not clearly defined, but spread throughout the precursor with a tendency to overlap with their six TMDs (Brix et al., 1999). Interestingly, the polytopic core proteins of the translocases of the inner membrane (Tim22, Tim17 and Tim23) are also substrates of the TIM22 complex, however they only contain four transmembrane domains (Figure 3) (Dekker et al., 1993; Maarse et al., 1994; Kübrich et al., 1994; Dudek et al., 2013).

Import along the carrier pathway can be dissected into several stages (Ryan et al., 1999). Initially the substrate is complexed by cytosolic chaperones to prevent aggregation of the hydrophobic transmembrane domains. This substrate-chaperone complex is then recognized by Tom70. One substrate might recruit six Tom70 receptors (three dimers) in order to facilitate complete takeover (Wiedemann et al., 2001). The release of the chaperones from Tom70 and the substrate requires ATP and leads to the transfer of the precursor to the Tom40 pore (Rapaport et al., 1997; Ryan et al., 1999).

Upon emergence of the precursor on the IMS side it is bound by the small Tim proteins, chaperoning the substrate through the aqueous environment (Sirrenberg et al., 1998). Carrier proteins are bound by the Tim9-Tim10 complex, while the Tim23 precursor is guided by the Tim8-Tim13 complex (Davis et al., 2007; Beverly et al., 2008). The Tim9Tim10-substrate complex is targeted to the TIM22 complex with the help of a fifth small Tim protein: Tim12. This complex docks to Tim54, a subunit of the TIM22 complex (Gebert et al., 2008). Tim54 is an inner membrane protein that contains a large IMS domain (Kerscher et al., 1997). Other subunits of the complex include Tim18 (which supports assembly of Tim54 into the complex), Sdh3 (which partners with Tim18) and Tim22 (the pore forming subunit) (Kerscher et al., 1997, 2000; Koehler et al., 2000; Kovermann et al., 2002; Gebert et al., 2011). After docking of the chaperone-substrate complex to Tim54 the precursor is treated in a two loop fashion into the twin-pore TIM22 translocase (Rehling et al., 2003). Opening of the pores requires the internal targeting signals of the carrier proteins as well as the membrane potential $\Delta \psi$. It is assumed that the translocation and insertion are driven by $\Delta \psi$, which acts on the positively charged residues in the matrix located loops. The mechanism of recognition, transport and release into the membrane remain to be elucidated in detail (Rehling et al., 2004). Subsequently, 
the imported single carrier subunits assemble into their mature complexes (Ryan et al., 1999).

Protein phosphorylation of translocase components has recently been discovered as a regulatory mechanism in mitochondrial import. The best studied example is reversible phosphorylation of Tom70 by protein kinase A. In fermentative metabolism using glucose, Tom70 is phosphorylated, which impairs recruitment of the chaperone-substrate complex and consequently import of carrier proteins (Schmidt et al., 2011; Rao et al., 2011).

\subsubsection{Protein export from the mitochondrial matrix into the inner membrane}

In addition to the import and sorting of nuclear encoded proteins, the core subunits of the respiratory chain (encoded by the mtDNA) need to be exported into the inner membrane. The central component of this machinery is the conserved Oxa1 (ortholog of the bacterial YidC) (Herrmann et al., 1997). All components of the translation machinery (ribosomes, mtDNA and translation activators) are associated with the inner membrane (Green-Willms et al., 2001). In contrast to the other proteins of the YidC family, Oxa1 contains a long C-terminal extension on the matrix side which binds ribosomes (Jia et al., 2003; Szyrach et al., 2003). Two additional proteins were linked to the recruitment of the ribosome to Oxa1: Mba1 and Mdm38 (Frazier et al., 2006; Ott et al., 2006). Hence it is believed that the insertion of the polytopic membrane proteins is co-translational, but the precise mechanism remains unclear (Hell et al., 1998; Stuart, 2002). An additional subunit, homolog to Oxa1, is involved in export: Cox18. It is implicated in the export of the C-terminus of Cox2, while Oxa1 is responsible for the export of the N-terminus (Saracco and Fox, 2002).

Another mechanism of protein export depends on the inner membrane AAA-ATPase Bcs1 (Nobrega et al., 1992). The ATPase domain faces the matrix side and allows translocation of a folded substrate (Rip1) across the inner membrane (Wagener et al., 2011; Wagener and Neupert, 2012). Rip1 is translated on cytosolic ribosomes and fully imported into the matrix via the presequence pathway. Subsequent to the acquisition of its $2 \mathrm{Fe}-2 \mathrm{~S}$ cluster and folding of the protein it is then translocated by the oligomeric Bcs1 across the inner membrane. ATP hydrolysis-dependent lateral release into the lipid phase occurs once the folded N-terminal domain has reached the intermembrane space. The Bcs1 
system can be seen as a Tat replacement in modern mitochondria (Wagener et al., 2011).

\subsubsection{Import of presequence-containing precursors - the TIM23 pathway}

\subsubsection{The N-terminal mitochondrial signal peptide - the presequence}

The presequence By far the largest class of precursor proteins $(70 \%)$ that are imported into mitochondria contain a presequence (Vögtle et al., 2009). The presequence is the classical N-terminal signal peptide for mitochondria and is not defined by a sequence motif but rather by its chemical properties (von Heijne, 1986). The presequence is 15 to 50 residues long and contains an amphipathic $\alpha$-helix (Allison and Schatz, 1986; Roise et al., 1986; Vögtle et al., 2009). One side of the helix is hydrophilic and the other hydrophobic, a feature that is then used for recognition by different receptors (described below). The presequence contains only few acidic residues and is net positively charged (von Heijne, 1986; Roise et al., 1986). The charge is essential for the initial transport across the inner membrane by the electrophoretic effect (Martin et al., 1991; Shariff et al., 2004).

Processing of presequence-containing precursors The presequence is typically cleaved in the matrix after import by the matrix processing peptidase (MPP) (Hawlitschek et al., 1988). Additional processing can occur by other peptidases after the MPP cleavage. The mitochondrial aminopeptidase (Icp55) as well as Oct1 remove destabilizing $\mathrm{N}$-terminal residues, thereby increasing the protein half-life according to the N-end rule (Vögtle et al., 2009, 2011). Some precursors contain a presequence that lacks the MPP cleavage site and are therefore not processed after import (e.g. Pam16).

The sorting signal A second route that releases soluble proteins into the intermembrane space uses the presequence pathway. A prerequisite for this is a sorting signal C-terminal of the presequence, which includes the transmembrane domain and the adjacent region (Botelho et al., 2011). The TMD is usually 16-18 residues long, longer segments tend to integrate into the outer membrane (Rojo et al., 1998). In the adjacent region negative charges or highly polar residues on the matrix side are disadvantageous due to the $\Delta \psi$, which is negative on the matrix side (Botelho et al., 2011). Conversely, 
positively charged residues on either side promote integration. After lateral release from the TIM23 complex into the lipid phase the precursor can be cleaved C-terminally of the TMD by the inner membrane peptidase (IMP) to release a solube IMS protein (Pratje and Guiard, 1986; Glick et al., 1992; Hahne et al., 1994).

Alternative signals Some proteins that are transported to the inner membrane by the TIM23 complex use unusual targeting signals. Bcs1 and Pam18 are for example targeted by a positively charged stretch located C-terminally of the TMD and are thought to be transported in a hairpin loop way through TIM23 enabling their $\mathrm{N}_{\text {out }} \mathrm{C}_{\text {in }}$ topology (Fölsch et al., 1996; Truscott et al., 2003). In contrast, the DNA helicase Hmi1 is imported in a C- to N-terminal direction through TIM23 with the C-terminal targeting signal being cleaved off afterwards. The signal has a propensity to form an $\alpha$-helix and is net positively charged (Lee, 1999). Recently, we showed that the polytopic inner membrane protein Sym1 is transported by TIM23 using internal targeting signals instead of a presequence (Reinhold et al., 2012).

\subsubsection{The TOM complex mediates passage across the outer membrane}

Assembly of the TOM complex The translocase of the outer membrane (TOM complex, see Figure 4.A and Table 1) is the general entry gate for protein import into mitochondria, almost all precursors pass through it. It is composed of the central pore forming subunit Tom40 (Hill et al., 1998), receptor subunits (Tom20, Tom22 and Tom70) (Hines et al., 1990; Schneider et al., 1991; Moczko et al., 1992; Hines and Schatz, 1993; Lithgow et al., 1994a), as well as small subunits important for assembly and stability (Tom5, Tom6 and Tom7) (Alconada et al., 1995; Hönlinger et al., 1996; Dietmeier et al., 1997; Claypool, 2009). The TOM complex is approx. $440 \mathrm{kDa}$ in size and forms two to three pores of $\sim 21 \AA$ as judged by electron microscopy (Künkele et al., 1998b,a; Hill et al., 1998; Ahting et al., 2001).

After the phosphatidylethanolamine-dependent translocation of the Tom40 precursor, the $\beta$-barrel is formed while it is engaged with the SAM complex (Becker et al., 2013; Qiu et al., 2013). Subsequently, the assembly of the TOM complex starts with the newly formed $\beta$-barrel of Tom40 at the SAM by association of Tom5 (Becker et al., 2010). The mitochondrial inner membrane organization system (MINOS) interacts with both the TOM and SAM complex and seems to be involved in early $\beta$-barrel biogenesis (Bohnert 
et al., 2012). Subsequently, the precursor of Tom6 associates with the Tom5-Tom40-SAM intermediate (Thornton et al., 2010). Association of Mdm10 with this intermediate leads to release of the TOM assembly (Yamano et al., 2010a). This interplay is complicated by the presence of Tom7, which recruits Mdm10 to the MMM1 complex (which tethers ER and mitochondria) and therefore prevents its assembly to the TOM-SAM intermediate. Additionally, Tom7 assembles to the TOM complex itself (Yamano et al., 2010b). Hence this provides a mechanism of regulated TOM release from the SAM complex. Afterwards, Tom40 and Tom 22 engage to form the core of the TOM complex, with Tom22 contacting two different Tom40 copies with either side of its transmembrane helix (Meisinger et al., 2001; Shiota et al., 2011). The cytosolic domain of Tom22 is needed for docking of Tom20 as well as Tom70 (van Wilpe et al., 1999; Yamano et al., 2008b). The biogenesis of the TOM complex is stimulated by phosphorylation of Tom22 by casein kinase 2 (Schmidt et al., 2011; Rao et al., 2011).

Tab. 1: Subunits of the translocase of the outer membrane (TOM complex)

\begin{tabular}{lll}
\hline \hline Subunit & Function $^{a}$ & Essential \\
\hline Tom40 & $\begin{array}{l}\text { protein channel, binds to presequence, keeps precursor } \\
\text { unfolded } \\
\text { Tom22 }\end{array}$ & yes \\
& $\begin{array}{l}\text { Tocognizes hydrophilic side of the presequence, platform for } \\
\text { Tom20 }\end{array}$ & no \\
recognizes the hydrophobic side of the presequence & no \\
Tom70 & $\begin{array}{l}\text { recognizes internal targeting signals, docks Hsp70, minor } \\
\text { role in presequence recognition }\end{array}$ & no \\
Tom5 & $\begin{array}{l}\text { presequence binding site on cis, transfer to the channel } \\
\text { Tom6 }\end{array}$ & no \\
Tom7 & $\begin{array}{l}\text { assembly and stability of TOM complex } \\
\text { presequence binding site on trans }\end{array}$ & no \\
& & no \\
\hline \hline
\end{tabular}

${ }^{a}$ detailed description as well as references can be found in the text

Transport of presequence substrates through TOM A recent study suggested that the cytosolic domains of both presequence receptors Tom20 and Tom22 are in close proximity to each other under resting conditions (Yamano et al., 2008b; Shiota et al., 2011). This is supported by findings in Saccharomyces castellii where the acidic N- 

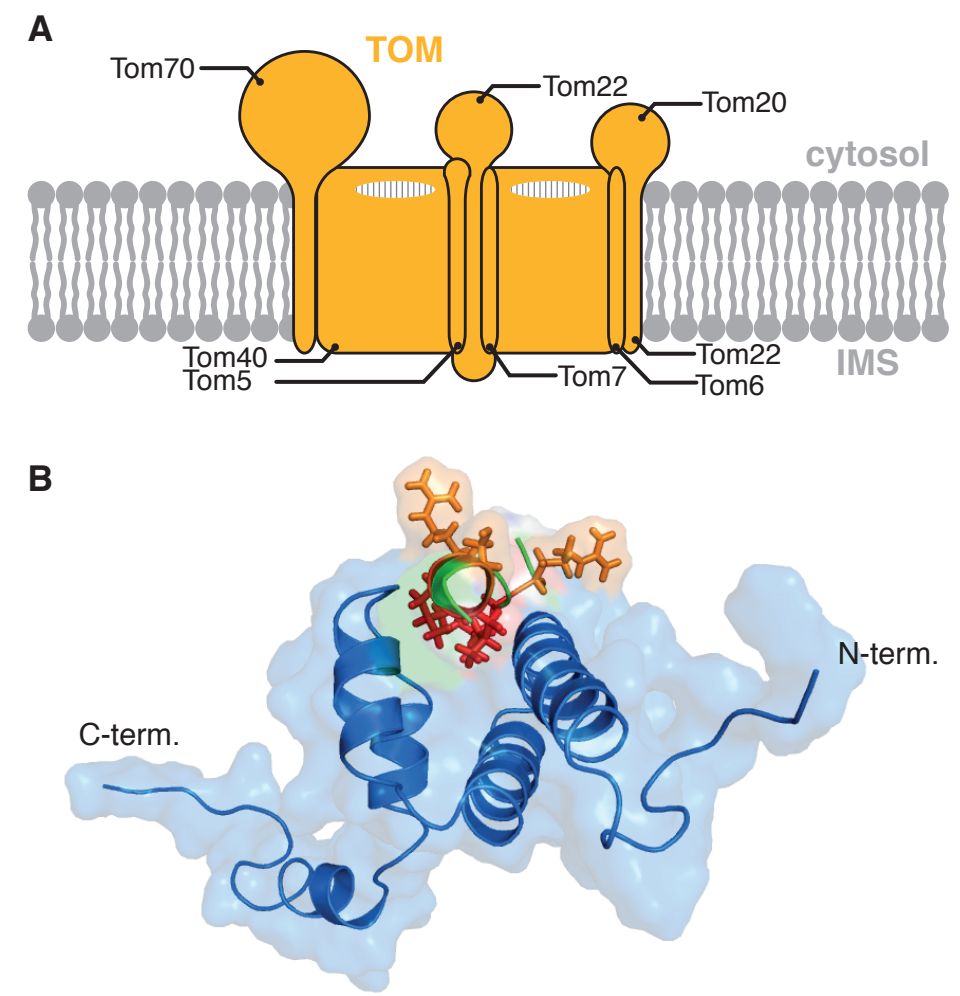

Fig. 4: The translocase of the outer mitochondrial membrane (TOM complex) A Schematic representation of the TOM complex and its subunits, only two of the three pores observed by electron microscopy are presented here. B Cartoon and surface representation of a NMR model of the interaction of rat Tom20 (blue) with rat aldehyde dehydrogenase presequence (green). Hydrophobic residues are colored in red while charged and polar residues are colored in orange (PDB ID: 1OM2, Abe et al. (2000).)

terminal domain of Tom22 is found at Tom20 C-terminus leading to the proposal of a composite receptor (Hulett et al., 2007). Recognition of the presequence by both receptors inserts the presequence into the Tom20-Tom22 clamp. Tom20 interacts with the hydrophobic face of the amphipathic presequence helix which has been studied extensively in atomic detail (Figure 4.B) (Abe et al., 2000; Muto et al., 2001; Obita et al., 2003; Komuro et al., 2013). In contrast, the cytosolic domain of Tom22 engages with the hydrophilic face of the presequence helix (Brix et al., 1997). All receptor subunits of the TOM complex possess a chaperoning activity (Wiedemann et al., 2001; Yano et al., 2004).

In addition to the classical presequence receptors, Tom70 has also been involved in the recognition of presequences as its interaction with presequence-containing substrates can be competeted with presequence peptides (Komiya et al., 1997). However this is debated, 
it seems as if Tom70 can engage with the mature part of some precursors to prevent their aggregation (Yamamoto et al., 2009).

From the Tom20-Tom 22 clamp the presequence is handed over to Tom5 on the cis side before it enters the channel formed by Tom40 (Dietmeier et al., 1997). In the Tom40 channel the precursor is kept in an unfolded state (Esaki et al., 2003). Interestingly, presequence import still functions after removal of the cytosolic domains of Tom20, Tom22 and Tom70 (Lithgow et al., 1994b; Dietmeier et al., 1997). It is this "bypass import" $(\sim 30 \%)$ that reflects the impact of the Tom5 on the import process.

During the transport of the presequence through the channel Tom40 binds to it (Rapaport et al., 1997). Once it emerges on the trans (IMS) side of the TOM complex it is bound by Tom40, Tom7 and potentially Tom22 ${ }^{\mathrm{IMS}}$ (Rapaport et al., 1997; Komiya et al., 1998; Kanamori et al., 1999; Esaki et al., 2004). The arrival of the presequence on the trans side is not necessarily coupled to unfolding of the substrate (Kanamori et al., 1999). Tom $22^{\mathrm{IMS}}$ is often referred to as a binding site for the presequence, however evidence for a direct interaction is still missing. It was only shown that presequence peptides prevent binding of the TOM complex to Tim $21^{\mathrm{IMS}}$, an interaction that occurs via Tom $22^{\mathrm{IMS}}$ (Chacinska et al., 2005; Albrecht et al., 2006).

It was proposed that the transport through the TOM complex is driven by a relay of interactions between the precursor and receptors. This involves hydrophobic as well as hydrophilic interactions with an increase in affinity towards the trans side (Komiya et al., 1998; Rehling et al., 2001).

\subsubsection{The TIM23 translocase drives transport across the inner membrane}

The TIM23 complex The translocase of the inner membrane (TIM23 complex, see Figure 5 and Table 2) is kept in close proximity to the TOM complex by interactions of Tim50 ${ }^{\mathrm{IMS}}$, and Tim21 $1^{\mathrm{IMS}}$ with Tom22 ${ }^{\mathrm{IMS}}$ (Figure 6) (Chacinska et al., 2005; Albrecht et al., 2006; Tamura et al., 2009; Shiota et al., 2011).

TIM23 exists in different forms (Figure 5). The core complex (TIM23 ${ }^{\mathrm{CORE}}$ ) is formed by the channel forming Tim23, its homolog Tim17, Tim50 and most likely Mgr2 (Chacinska et al., 2005, 2009, 2010; Wiedemann et al., 2007; Gebert et al., 2012). Tim23 forms the hydrophilic channel in the inner membrane and opens in the presence of a $\Delta \psi$ and presequence peptides (Lohret et al., 1997; Truscott et al., 2001; van der Laan et al., 2007; Meinecke et al., 2006; Alder et al., 2008a). During transport as well as at resting state the 
channel lumen is not accessible from the IMS, indicating that the pore is sealed (Alder et al., 2008a).

Tim23 contains a 100 amino acid long soluble IMS domain. The first half has been proposed to span the outer membrane (Donzeau et al., 2000; Popov-Celeketić et al., 2008a; Gevorkyan-Airapetov et al., 2009; Tamura et al., 2009), although the functional importance has been debated (Chacinska et al., 2003, 2005). The second half recognizes the presequence and interacts with the IMS domain of Tim50 (Bauer et al., 1996; Geissler et al., 2002; Yamamoto et al., 2002; Tamura et al., 2009; de la Cruz et al., 2010). The opening of Tim23 in the absence of presequence substrate and hence leakage of the $\Delta \psi$, is blocked by Tim50 ${ }^{\mathrm{IMS}}$ which induces channel closure (Meinecke et al., 2006). Tim50 ${ }^{\mathrm{IMS}}$ binds to Tim23 ${ }^{\text {IMS }}$ using a $\beta$-loop $\left(K_{\mathrm{D}} 60 \mu \mathrm{M}\right)$ (Alder et al., 2008b; Gevorkyan-Airapetov et al., 2009; Qian et al., 2011). Additionally, Tim50 is in close proximity to TMD1 of Tim23. This interaction is enhanced by a $\Delta \psi$ collapse which might lift TMD1 partially out of the bilayer. A possible result could be an enhanced channel closure to protect the remaining $\Delta \psi$ (Alder et al., 2008b).

Apart from Tim50 binding, the IMS domain of Tim23 also mediates a $\Delta \psi$ dependent contact to a second Tim23 molecule via its putative leucine zipper. This interaction is dissociated in the presence of a presequence (Bauer et al., 1996; Alder et al., 2008b). Upon depolarization the TIM23 channel opens up, exposing large parts of TMD2 to the intermembrane space (Malhotra et al., 2013). This change induces a tilt at a conserved tyrosine in the TMD2 with the C-terminal part of the helix losing its secondary structure (Malhotra et al., 2013).

Tim17 is homolog to Tim23, but it lacks the IMS domain (Kübrich et al., 1994; Maarse et al., 1994). It has been implicated in the gating of the translocase and sorting of precursors into the inner membrane (Chacinska et al., 2005; Meier et al., 2005a; MartinezCaballero et al., 2007; Chacinska et al., 2010). In the resting state (energized membrane) the TMD1 of Tim23 is in contact with TMD4 of one or two molecules of Tim17 (Alder et al., 2008b). The presence of a precursor or the depletion of $\Delta \psi$ interrupt this interaction.

Tim23 is referred to as the pore forming subunit, based on the findings that purified Tim23 from E. coli can be reconstituted to form a channel (Truscott et al., 2001). Additionally, in tim23-2 mutants the channel is still formed although it does not contain Tim17 anymore (Truscott et al., 2001). As both Tim23 and Tim17 contain four transmembrane domains, the protein-conducting channel is probably composed of two to three subunits 
of Tim23 and optionally Tim17. The impact of Tim17 on the gating and structure of the channel implies that the pore can exist in a Tim23-only or a mixed Tim23-Tim17 state (Martinez-Caballero et al., 2007).

Recently, the two transmembrane span containing inner membrane protein Mgr2 was identified as a new TIM23 subunit (Gebert et al., 2012). Just like Tim23 and Tim17, Mgr2 is in close proximity to a translocated precursor (Popov-Celeketić et al., 2011; Gebert et al., 2012). Taking the position of the predicted TMDs as well as the processing of the C-terminal 33 amino acids into account (R. Ieva and M. van der Laan, personal communication), Mgr2 is most likely a TIM23 ${ }^{\mathrm{CORE}}$ component lacking soluble domains at the $\mathrm{N}$ - and C-terminus.

It should be noted that the stoichiometry of the different subunits within the TIM23 is not known. There is currently no evidence for a long-lived TIM23 ${ }^{\mathrm{CORE}}$, the default state of the translocase rather seems to be the sorting form. TIM $23^{\mathrm{SORT}}$ is composed of the core complex and the additional Tim21 that is coupled by Mgr2 (Chacinska et al., 2005; van der Laan et al., 2006; Chacinska et al., 2010; Gebert et al., 2012). Furthermore, Tim21 ${ }^{\text {IMS }}$ binds to Tim $23^{\mathrm{IMS}}$ with a $K_{\mathrm{D}}$ of $1 \mu \mathrm{M}$ (Lytovchenko et al., 2013). This coupling enables the TIM $23^{\text {SORT }}$ to be associated with the respiratory chain by the interaction of Tim21 with Qcr6 of complex III, which is thought to be beneficial for initial transport steps across the inner membrane due to the large local $\Delta \psi$ (van der Laan et al., 2006).

Recently, it was shown that Tim50 ${ }^{\mathrm{IMS}}$ and Tim $21^{\mathrm{IMS}}$ interact with each other with high affinity $\left(K_{\mathrm{D}} 260 \mathrm{nM}\right)$ (Lytovchenko et al., 2013). During this interaction the cysteines of both domains are in close proximity (Figure 6). This interaction is facilitated by Tim23 and opens up in the presence of presequence peptides and Tim23 (Lytovchenko et al., 2013). The liberation of Tim21 leads to an exchange of Tim21 for Pam17, which triggers import motor assembly.

The transport pathway for matrix targeted precursors The current model of presequence import across the TIM23 complex starts with the transfer of the presequence bound to Tom $22^{\mathrm{IMS}}$ to the TIM23 complex. This is most likely mediated by either an indirect competition of Tim $21^{\mathrm{IMS}}$ with the presequence for the binding site on Tom $22^{\mathrm{IMS}}$ or a direct competition of Tim $50^{\mathrm{IMS}}$ and Tom $22^{\mathrm{IMS}}$ for the presequence (Chacinska et al., 2005; Mokranjac et al., 2005a; Shiota et al., 2011). Subsequently, the precursor is bound by Tim50 (Geissler et al., 2002; Yamamoto et al., 2002; Mokranjac et al., 2003a). It is 


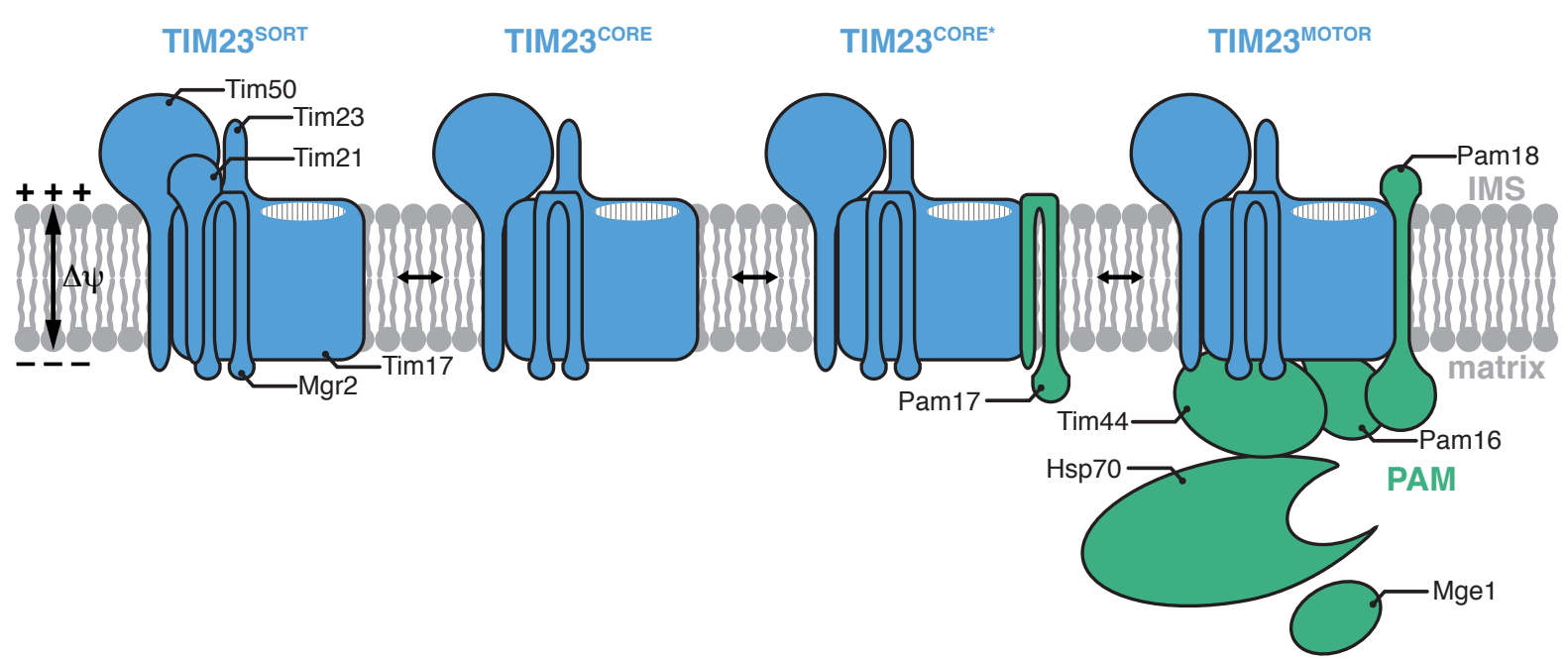

Fig. 5: The translocase of the inner mitochondrial membrane (TIM23 complex) and the associated import motor (PAM) - Schematic representation of the TIM23 (blue) and PAM complex (green) and their subunits. The Tim21 containing TIM23 $3^{\text {SORT }}$ can associate with complex III via Qcr6 which is not depicted here. Tim23 and Tim17 are depicted as one subunit.

Tab. 2: Subunits of the presequence translocase (TIM23 complex)

\begin{tabular}{lll}
\hline \hline Subunit & Function $^{a}$ & Essential \\
\hline Tim23 & $\Delta \psi$ and presequence dependent protein channel, recognizes & yes \\
& presequence, binds to Tim17, Tim21 and Tim50 & \\
Tim17 & involved in gating and sorting, tethers Pam18 & yes \\
Tim50 & binds to presequence precursor, transfer from TOM to & yes \\
& TIM23, induces channel closure under resting conditions & \\
Mgr2 & couples TIM23 & no \\
Tim21 & couples TIM23 & Tim21 \\
\hline \hline
\end{tabular}

${ }^{a}$ detailed description as well as references can be found in the text

then transferred to Tim $23^{\mathrm{IMS}}$, which binds to the presequence with its C-terminal half of the IMS domain $\left(K_{\mathrm{D}} 0.5 \mathrm{mM}\right)$, and guided into the channel of the TIM23 complex (de la Cruz et al., 2010; Lytovchenko et al., 2013). The transport across the inner membrane is driven by the $\Delta \psi$ acting on the net positively charged presequence (electrophoretic effect) (Martin et al., 1991; Shariff et al., 2004). Interestingly, the transfer of precursor proteins from TOM to TIM23 and the subsequent coupling of both translocases is efficient enough 
A
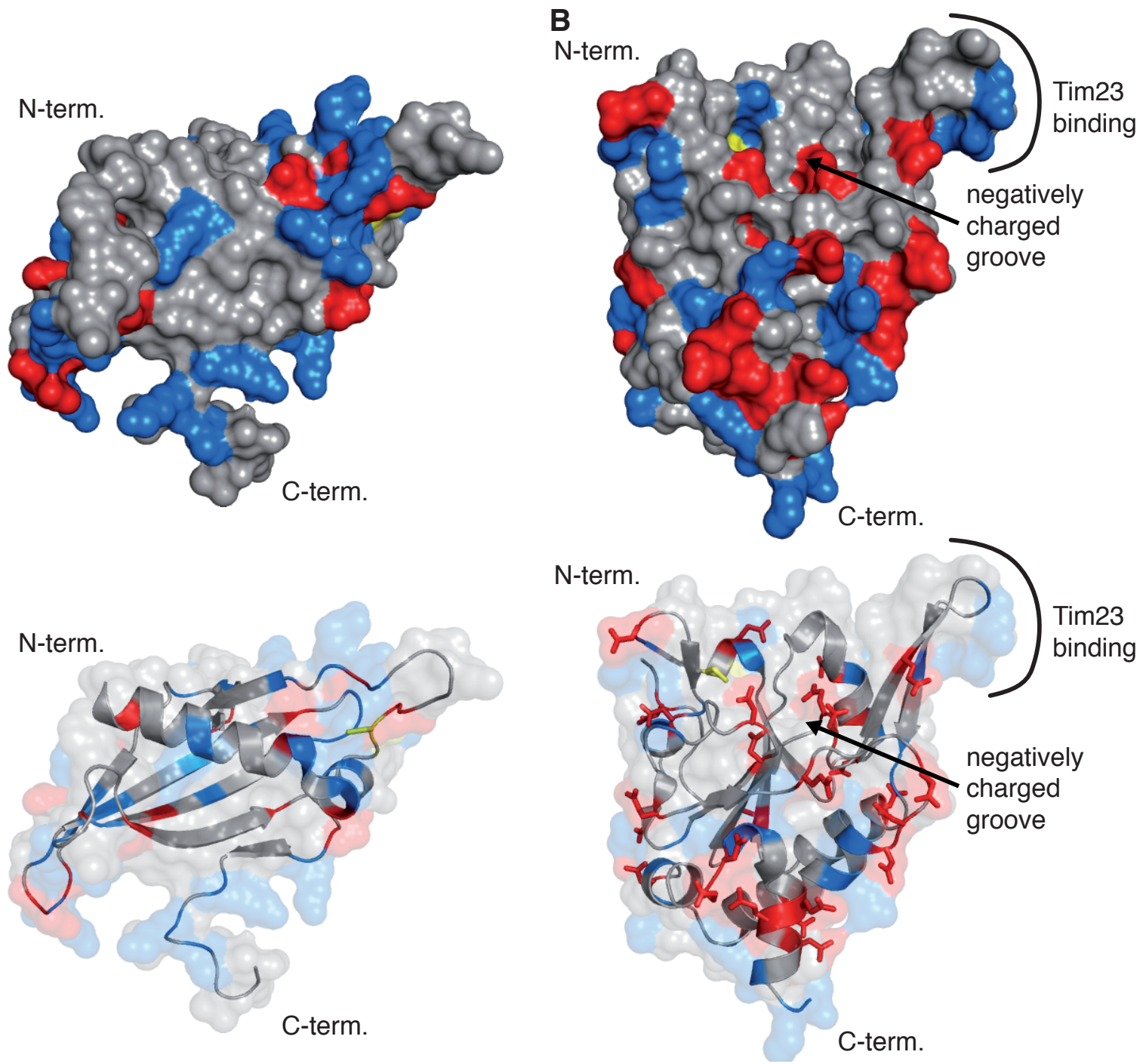

Fig. 6: Atomic models of Tim21 ${ }^{\mathrm{IMS}}$ and Tim50 $0^{\mathrm{IMS}}$ - A Surface (upper panel) and cartoon representation (lower panel) of Tim21 ${ }^{\mathrm{IMS}}$. Positive, negative and cysteine residues are colored in blue, red and yellow, respectively (PDB ID: 2CIU, Albrecht et al. (2006)). B Surface (upper panel) and cartoon representation (lower panel) of Tim50 ${ }^{\mathrm{IMS}}$. Coloring as in A. The $\beta$-hairpin responsible for Tim $23^{\mathrm{IMS}}$ binding as well as the negatively charged groove proposed to responsible for presequence binding are indicated (PDB ID: 3QLE, Qian et al. (2011)). 
to prevent readout of later targeting signals in the precursor (Maruyama et al., 2012).

Besides Tim23 no other presequence receptor in the TIM23 complex was identified so far, however Tim50 is believed to fulfill this function. Even though Tim50 ${ }^{\mathrm{IMS}}$ induces closure of the Tim23 channel, presequence peptides can open it again indicating a recognition event (Meinecke et al., 2006). It is unclear whether this is solely due to the recognition of the presequence by Tim $23^{\mathrm{IMS}}$. Recent atomic insight into the structure of the intermembrane space domain of Tim50 revealed a negatively charged groove, which was proposed to be responsible for presequence binding (Figure 6.B) (Qian et al., 2011).

It should be noted that apart from the modular model of the TIM23 complex described here, a single-entity model is also discussed. This argues that the import motor and Tim21 are always associated with the TIM23 translocase (Tamura et al., 2006; PopovCeleketić et al., 2008a, 2011). Recently, it was suggested that all results seen so far reproduced different states of the TIM23-PAM reaction cycle leading to the different models (Chacinska et al., 2010).

The sorting pathway After the presequence is transported across the inner membrane the translocase can engage a stop transfer signal in the precursor (see 1.2.5.1) (van Loon et al., 1986; Glaser et al., 1990; Glick et al., 1992). Sorting into the inner membrane was demonstrated with the TIM23 ${ }^{\text {SORT }}$ complex (van der Laan et al., 2007). Despite the channel forming Tim23, Tim17 was shown to be actively involved in the sorting process (Chacinska et al., 2005). The coupling of the translocase to the respiratory chain by Tim21 makes the sorting process more resistant to changes in the membrane potential (van der Laan et al., 2006). Interestingly, the sorting and matrix transport by TIM23 seem to be inversely regulated as overexpression of Tim21 reduces matrix import (Chacinska et al., 2005; Popov-Celeketić et al., 2008a). This is most likely due to a reduction of PAM subunits at TIM23 (Chacinska et al., 2010). Additionally, Tim17 mutants defective in motor association, or Pam18 mutants deficient in association with Tim17 lead to an increased sorting efficiency (Chacinska et al., 2005, 2010; Popov-Celeketić et al., 2011).

It remains elusive, whether the $\Delta \psi$ dependence of the sorting process is only due to the transport of the presequence across the membrane or also the lateral release itself. The hydrophobic (TMD) part of the sorting signal is arrested in the channel (Gruhler et al., 1997), and afterwards the substrate partitions into the lipid bilayer. Interestingly, the import motor components Pam16 and Pam18 were found in close vicinity to laterally 
sorted substrates and they affect the efficiency of insertion independent of the ATPase activity of the import motor (Popov-Celeketić et al., 2011).

The limiting step for complete import is the subsequent unfolding of the C-terminal part and its import through TOM, which could be driven by the subsequent refolding of the C-terminal domain in the intermembrane space (Gruhler et al., 1997; Rojo et al., 1998).

In addition to the stop transfer pathway, a conservative sorting mechanism is known (Hartl et al., 1987). Here the precursor is first completely imported into the mitochondrial matrix and subsequently exported into the inner membrane using OXA or Bcs1 (see section 1.2.4) . Differentiation between both pathways is achieved at the level of the sorting signal. Stop transfer signals are more hydrophobic and contain no proline residues (Meier et al., 2005b). Furthermore the cluster of charged amino acids in the adjacent region seems to contribute to a stop transfer (Rojo et al., 1998). The differentiation between both sorting pathways can depend of the state of the cell and its growth conditions (Park et al., 2013).

A particular case of inner membrane sorting is the biogenesis of some polytopic membrane proteins (e.g. Mdl1). The initial two transmembrane domains of Mdl1 are sorted by the TIM23 complex, while the third and fourth are imported into the matrix and exported by the OXA complex. Finally the last two TMD are again laterally sorted by the TIM23 complex (Bohnert et al., 2010). The properties of TMD three and four are more similar to the TMDs of conservatively sorted substrates, they are only moderately hydrophobic and contain proline residues.

\subsubsection{Full matrix translocation is powered by the PAM complex}

Full translocation into the mitochondrial matrix depends on the presequence translocase associated motor (PAM complex). This ATP driven import motor is one of the most complex Hsp70 based systems (Mayer, 2004).

Hsp70 systems While the main function of Hsp70 proteins is protein folding, they are also associated with protein translocation in different cellular compartments like the ER, chloroplasts or mitochondria (Matlack et al., 1999; Chacinska et al., 2009; Flores-Pérez and Jarvis, 2013). 
Hsp70 chaperones share a conserved domain structure: an N-terminal nucleotide binding domain (NBD) followed by an interdomain linker and the C-terminal peptide binding domain (Hartl and Hayer-Hartl, 2002). The mechanism of Hsp70 function in folding is well studied in the bacterial DnaK system (Liberek et al., 1991; Langer et al., 1992; Zhu et al., 1996; Russell et al., 1998). In the ATP bound state the affinity of the peptide binding domain for substrate peptides is low and the on-off rate is high, enabling the chaperone to bind rapidly to new substrates. However, in order to associate tightly with the substrate, ATP hydrolysis is needed. The intrinsic ATPase activity is usually low and requires external stimulation by DnaJ (Hsp40).

J-proteins posses a typical four helical fold with a conserved HPD motif in the loop between helix II to helix III. The interaction between Hsp40 with Hsp70 is mediated by parts of helix II, the HPD motif and helix III of Hsp40 with the interdomain linker and proximal residues of Hsp70 (Greene et al., 1998; Jiang et al., 2005, 2007). Binding of the J-protein to Hsp70 changes the interaction of the NBD with the peptide binding domain and alters the conformation of the interdomain linker. This is then transmitted to the active site in the NBD leading to an enhanced ATP hydrolysis (Jiang et al., 2007; Swain et al., 2007). In the ADP bound state the peptide binding domain has a high affinity for its substrate with a slow on-off rate. In this state the Hsp70 assists in protein folding or generates vectorial protein translocation. For repeated Hsp70 binding ADP needs to be released by a GrpE class protein followed by binding of a new ATP molecule.

All these co-chaperones needed for a Hsp70 system are found in the mitochondrial matrix. However there is a redundancy: besides the main Hsp70 (mtHsp70, Ssc1 in yeast) other Hsp70 proteins ( $\mathrm{Ssq} 1$ and $\mathrm{Ssc} 3$ ) can be found. The same is true for J-proteins: Pam18 functions in protein import while the soluble Mdj1 is involved in the protein folding (Rowley et al., 1994; D'Silva et al., 2003). The soluble Mge1 is the nucleotide exchange factor in the mitochondrial matrix (Laloraya et al., 1994; Schneider et al., 1996; Miao et al., 1997; Schmidt et al., 2001). It should be noted that a second J-protein can be found at the translocase: Mdj2, however it is not essential for cell growth and protein import and is not a not a stoichiometric component of the PAM complex, hence its role in mitochondrial import remains elusive (Westermann and Neupert, 1997; Mokranjac et al., 2005b).

The dynamics of Ssc1 conformation have been studied extensively (Mapa et al., 2010; Sikor et al., 2013). Similar to the bacterial system described above, ATP bound Ssc1 
can bind to its substrate upon Mdj1 stimulated ATP hydrolysis that induces closure of the lid of the substrate binding domain. The association of Mdj1 with this substrate bound ADP-Ssc1-complex is surprisingly stable, as it can remain to be bound while Mge1 already triggered nucleotide release and therefore ATP rebinding (Mapa et al., 2010). In the substrate free state, Ssc1 can fluctuate between an ATP state with an open substrate binding domain lid and an ADP or nucleotide free state with a flexible lid (Sikor et al., 2013).

Interestingly, the mitochondrial Hsp70 depends on itself as well as an additional chaperone during its biogenesis. After motor dependent import into the mitochondrial matrix, Hsp70 exists in an unfolded state. Folding of the peptide binding domain functions without any additional factors, however folding of the NBD has to be assisted by Hep1 (Tim15) (Burri et al., 2004; Sanjuán Szklarz et al., 2005; Sichting et al., 2005; Yamamoto et al., 2005). Hep1 is a zinc finger protein and requires the presence of $\mathrm{Zn}^{2+}$ for its own folding (Fraga et al., 2013). The intermediate Hsp70-Hep1 complex can then bind nucleotides to the NBD, stabilizing its fold and releasing Hep1. During its life cycle nucleotide-free Hsp70 can undergo transformation into an aggregation prone intermediate, which can be rescued again by Hep1 (Blamowska et al., 2010, 2012). This mechanism is also found in chloroplasts, and hence might serve to prevent folding and activation of the Hsp70 molecules in the cytosol, but only in their respective compartment where the appropriate Hep1-type chaperone is present (Blamowska et al., 2012).

The PAM complex The import motor promotes ATP dependent translocation of precursors into the matrix and is also needed for the import of sorted proteins when the sorting signal is not adjacent to the presequence (e.g. Cox5a) (Figure 5 and Table 3) (Gärtner et al., 1995). These precursors are initially imported in a motor dependent manner but are sorted as soon as the sorting signal is presented to the TIM23 complex. This implies that the mechanism of sorting of inner membrane proteins involves a dynamic conversion of TIM23 $3^{\text {MOTOR }}$ into TIM23 $3^{\text {SORT }}$ during the import process.

The initial steps of presequence transfer across the inner membrane, depend on Pam17 that is recruited to TIM23 by interactions with Tim17 (van der Laan et al., 2005; Schiller, 2009). Later, Pam17 initiates the assembly of the J-module at the translocase, and has been involved in the regulation of the conversion of the TIM23 translocase between the matrix transport and lateral insertion mode (van der Laan et al., 2005; Hutu et al., 2008; 
Popov-Celeketić et al., 2008a; Schiller et al., 2008).

As the presequence emerges on the matrix side it is bound by Tim44 ( $\left.K_{\mathrm{D}} 230 \mathrm{nM}\right)$ (Marom et al., 2011). Tim44 itself binds to the inner membrane, especially to cardiolipin, the specific phospholipid of mitochondria, using a large hydrophobic pocket (Weiss et al., 1999; Josyula et al., 2006; Handa et al., 2007; Marom et al., 2009; Schlame, 2013). The protein associates with TIM23 via interactions with Tim23 and Tim17 (Berthold et al., 1995). After this initial trapping of the precursor, which prevents backsliding, the precursor is than engaged by Hsp70. The chaperone is recruited to the exit site of the TIM23 complex by Tim44 which acts as a molecular scaffold (Blom et al., 1993; Kronidou et al., 1994; Schneider et al., 1994; Hutu et al., 2008). Tim44 and Pam17 seem to work in an antagonistic manner (Hutu et al., 2008; Popov-Celeketić et al., 2008a).

Continuous binding of Hsp70 to the substrate is stimulated by the activation of the ATPase activity by the J-protein Pam18 (D'Silva et al., 2003; Mokranjac et al., 2003b; Truscott et al., 2003). Pam18 is an inner membrane protein with its C-terminal J-domain being exposed on the matrix side. It is recruited to the TIM23 complex by three separate interactions: the TMD as well as interactions of its N-terminal IMS domain with Tim17 and a complex of its J-domain with the J-like domain of Pam16 (Figure 7) (Frazier et al., 2004; Kozany et al., 2004; Li et al., 2004; Chacinska et al., 2005; Iosefson et al., 2007; Mokranjac et al., 2007; D'Silva et al., 2008). Pam16 is not able to stimulate the ATPase activity of Hsp70, even when the HPD motif is introduced by mutagenesis (Frazier et al., 2004; Kozany et al., 2004; Li et al., 2004). Pam16 is tethered to the inner membrane by interactions of the hydrophobic N-terminus with the N-terminal region of Tim44 (Schilke et al., 2012).

Mechanistic impact of the interaction within the J-complex has been debated for some time. It was widely accepted that the inhibition of Pam18s stimulation of Hsp70 by Pam16 (by $\sim 50 \%$ ) could prevent futile cycles at the translocase exit site (Frazier et al., 2004; Li et al., 2004; Chacinska et al., 2005; Neupert and Herrmann, 2007; Mokranjac and Neupert, 2010; van der Laan et al., 2010; Endo et al., 2011). This was further supported by the atomic structure of the J-complex, which revealed that residues that would be important for the Pam18-Hsp70 interaction are occupied in the J-complex by Pam16 (Mokranjac et al., 2006).

However, this model is challenged by the view that the interaction of Pam16 and Pam18 has no regulatory role, but only serves to position Pam18 at the translocase 
exit site (Schilke and Craig, 2011). This was based on genetic analysis that shows that mutants with a reduced stimulatory capacity show no reduced growth. It should however be noted that growth phenotypes are usually a poor indicator of the mitochondrial import capacities, as even strong reductions by $80 \%$ would not be visible in a growth test (Tamura et al., 2009). Furthermore it was suggested that the regulatory role is rather carried out by the interaction of the N-termini of Tim44 and Pam16, which could modulate the relative positioning of Pam18 and Hsp70 to each other (Schilke and Craig, 2011; Schilke et al., 2012). This would imply a mechanism for Tim44 or Pam16 to sense the incoming polypeptide. Such a mechanism is highly speculative, but could be started by the recognition of the presequence by Tim44 and a signal transmission across the membrane might involve Tim17 and Pam17 (Marom et al., 2011; Schilke et al., 2012).

The J-module interacts with the respiratory chain, just like TIM23 ${ }^{\mathrm{SORT}}$, however independent of Tim21 (Wiedemann et al., 2007). The function might be the coordinated switch from TIM23 $3^{\text {SORT }}$ to TIM23 $3^{\text {MOTOR }}$. It should however be noted that the distribution of the different complexes in the inner mitochondrial membrane seems to be different. While the respiratory chain supercomplexes are largely found in the cristae, TIM23 should maintain contact sites with TOM at the inner boundary membrane (Vogel et al., 2006; Zick et al., 2009).

In conclusion the exact role of the Pam16-Pam18 complex (J-module) is not yet completely understood. It clearly serves in recruitment of Pam18 to close vicinity of the exit site of TIM23, which together with the Hsp70 recruitment is important for an efficient and spatially controlled activation of the ATPase activity.

Vectorial movement of the precursor Two major models for the generation of vectorial movement during mitochondrial import have been proposed: the "Brownian ratchet mechanism" (Simon et al., 1992; Schneider et al., 1994) and the "power stroke mechanism" (Pfanner and Meijer, 1995; Glick, 1995). The hallmark of the "power-stroke model" is that mtHsp70 undergoes a conformational change, which is powered by the ATP hydrolysis and uses Tim44 as a fulcrum to generate a force directed into the matrix. However, this hypothesis lacks direct experimental evidence.

The ratchet or trapping mechanism functions by several rounds of mtHsp70 binding to the precursor emerging from the translocation pore and preventing backsliding. The import is therefore driven by the Brownian thermal motion of the precursor in the 


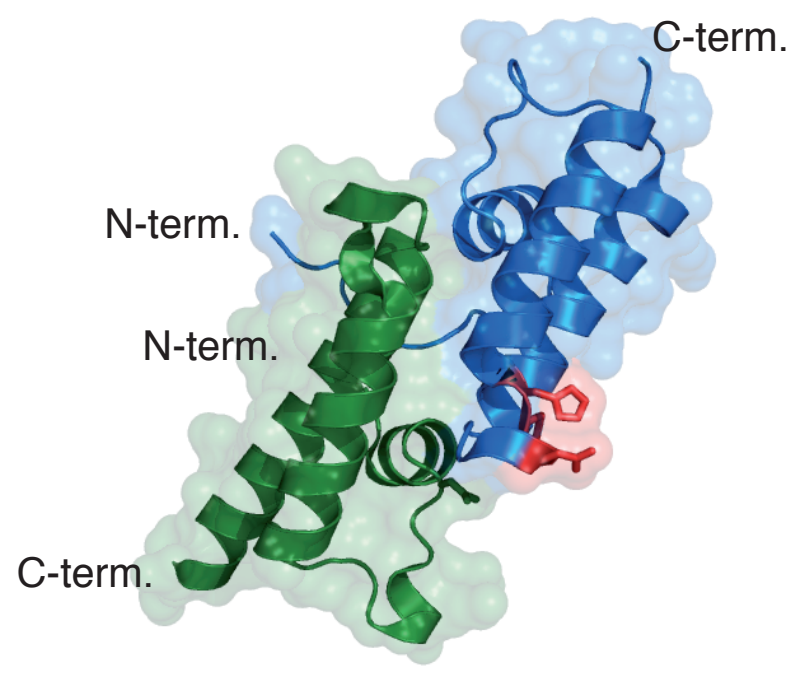

Fig. 7: Atomic model of the Pam16-Pam18 interaction (J-complex) - Cartoon representation of the J- and J-like domains of Pam18 (blue) and Pam16 (green) in complex with each other. The signature HPD motif of Pam18 is colored in red (PDB ID: 2GUZ, Mokranjac et al. (2006)).

translocation channel. As the bound Hsp70 prevents backsliding the precursor can only move into the mitochondrial matrix exposing another segment that can be engaged by a new Hsp70 molecule. Hence, the asymmetric distribution of mtHsp70 molecules with respect to the inner membrane and the ATP driven binding of mtHsp70 to the substrate contribute to the movement. The unfolding of tightly folded domains on the surface of mitochondria represents the limiting step in import and hence determines the import efficiency (Gaume et al., 1998). Evidences like the substrate binding induced release of mtHsp70 from Tim44 as well as a the precursor-dependent step-size argue in favor of this model as a minimal mechanism (Schneider et al., 1996; Okamoto et al., 2002; Liu et al., 2003; Yamano et al., 2008a).

This model was recently extended to include thermodynamic aspects of the translocation (entropic pulling) (Rios et al., 2006). Binding of the mtHsp70 molecules to the substrate decreases the available diffusion volume of the mtHsp70 as membrane proximal regions are excluded. Hence diffusion is directed towards the matrix providing a force to unfold protein domains on the mitochondrial surface.

Based on the ratchet and entropic pulling model, the following mechanism of vectorial movement is accepted (Figure 8) (Neupert and Herrmann, 2007; Chacinska et al., 2009; 
Tab. 3: Subunits of the presequence translocase associated import motor (PAM complex)

\begin{tabular}{lll}
\hline \hline Subunit & Function $^{a}$ & Essential \\
\hline Tim44 & binds to precursor, tethers Hsp70 to exit site & yes \\
Hsp70 & ATP dependent precursor binding, generates vectorial & yes \\
(Ssc1) & movement & \\
Pam16 & tethers Pam18 to the exit site of the translocase, binds with & yes \\
& N-terminus to Tim44 N-terminus & \\
Pam17 & assembly of Pam16-Pam18 complex, involved in initial & no \\
& transfer steps & \\
Pam18 & J-protein, activates ATPase activity of Hsp70, tethered by & yes \\
& Pam16 and interactions of its IMS domain with Tim17 & \\
Mge1 & nucleotide exchange factor of Hsp70 & yes \\
Hep1 & assists folding and stability of Hsp70 & yes \\
\hline \hline
\end{tabular}

${ }^{a}$ detailed description as well as references can be found in the text

Mokranjac and Neupert, 2010). The first critical step, is the recruitment of ATP-bound mtHsp70 to Tim44. This is stimulated by the nucleotide exchange factor Mge1 (Schneider et al., 1996; Liu et al., 2003). Once positioned correctly at the translocase, Hsp70ATP engages with the precursor. ATP hydrolysis in close vicinity to the exit site of the translocase is triggered by Pam18 (D'Silva et al., 2003; Mokranjac et al., 2003b; Truscott et al., 2003), it is not known whether a successful binding of the substrate by Hsp70 is stimulated by this mechanism or whether this is solely regulated by the positioning of the subunits in the first place. The dissociation of Hsp70 ADP from Tim44 is facilitated by Mge1 and release of inorganic phosphate (Schneider et al., 1996). At this step the vectorial movement takes place, as rebinding of the Hsp70 $\mathrm{ADP}$ to Tim44 is very inefficient, it diffuses away into the matrix (Schneider et al., 1996; Liu et al., 2003; Mokranjac et al., 2003b). The free Tim44 can bind another Hsp70 ${ }_{\text {ATP }}$ that can then efficiently trap the newly exposed segment of the precursor (Moro et al., 1999). The first Hsp70 ${ }_{\mathrm{ADP}}$ undergoes release of the ADP triggered by Mge1 (Laloraya et al., 1994; Schneider et al., 1996; Miao et al., 1997). Due to the high ATP concentration in the matrix, ATP is bound again to Hsp70 liberating the substrate. The step-size of the Hsp70 can vary between 20 to 60 residues, depending on the unfolding rate of the precursor protein (Yamano et al., 2008a). It is not known 
so far whether any of the other PAM subunits follow the cyclic recruitment of Hsp70, or whether the subunits are firmly engaged with the translocase during translocation of one precursor. Successive rounds of this cycle will eventually complete matrix translocation of the precursor. The import rates can reach 10-15 pmol imported protein/ min /mg mitochondria in vitro, meaning that one TIM23 takes 1 min to transport one precursor (Milisav et al., 2001; Ryan et al., 2001).

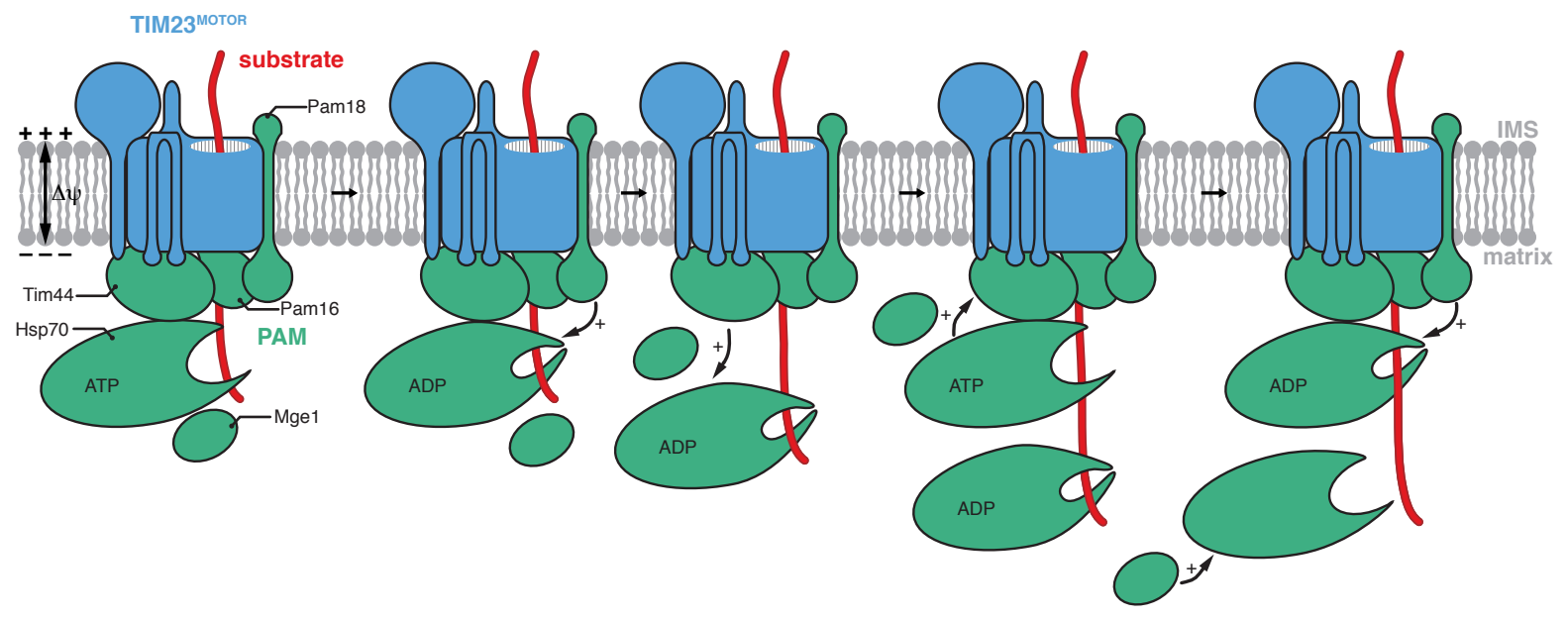

Fig. 8: The Hsp70 cycle during protein import - Schematic representation of the Hsp70 cycle during protein import into the mitochondrial matrix. Tight binding of Hsp70 to the substrate at the translocase is triggered by Pam18 mediated stimulation. Hsp70ADP dissociates from Tim44 (facilitated by Mge1) and vectorial movement of the precursor is achieved by Brownian motion. The free Tim44 is occupied by a new Hsp70 ATP (faciliated by Mge1). Release of the ADP from the first Hsp70 is mediated by the nucleotide exchange factor Mge1 leading to a dissociation of the Hsp70 from its substrate. A prerequisite for efficient import is that the ATP hydrolysis of the new Hsp70 at the translocase does not occur significantly later than the substrate release from the old Hsp70 to prevent backsliding. 


\subsection{Aim of this work}

The presequence translocase in the inner mitochondrial membrane (TIM23 complex) is an essential protein complex. It translocates and sorts a diverse set of substrate proteins into the matrix or inner membrane. Fruitful research in the recent decades discovered a wide set of subunits of the TIM23 complex as well as the associated import motor (PAM complex). Furthermore a functional separation of the sorting process, performed by the TIM23 $3^{\text {SORT }}$, as well as the matrix import, performed by TIM23 $3^{\text {MOTOR }}$, was reported.

However, the precise sequence of events leading to the transfer of the precursor from the TOM to the TIM23 complex is not very well understood, especially concerning the recognition of the presequence. This is in strong contrast to the translocase of the outer membrane (TOM complex) where recognition events with several subunits have been reported. Hence, the first aim of this study was to develop new tools to identify presequence binding proteins. Furthermore the molecular analysis of the precise binding site on a potential receptor would facilitate studies of its contribution to the import process. From this better mechanistic understanding of the ill-defined steps of presequence transfer from the TOM to the TIM23 might be possible.

The versatility of the TIM23 complex is reflected by the plethora of different subunits required for proper function of the translocase. This is especially true for the import motor, which consists of seven different subunits and exits in excess over the TIM23 complex in the inner membrane. The currently proposed mechanism involves cycling of the Hsp70 during the ratcheting of the precursor. The complex regulation of the Hsp70 activation is already anticipated, however it is unclear whether other subunits follow the recruitment cycles of Hsp70. Or, more generally put: whether the translocase is a stable entity during the translocation of one precursor, or whether subunits exchange within the active translocase during the transport process and how that affects the regulation of Hsp70 activity. 


\section{Results}

\subsection{Identification of presequence binding proteins and characterization of the respective domains}

\subsubsection{Presequence photopeptides}

\subsubsection{Design of photopeptides}

Structural information about the interactions of presequences with their receptors during translocation of a substrate into mitochondria is limited. The only example with atomic resolution is the interaction of Tom20 in complex with the presequence of the aldehyde dehydrogenase presequence (Figure 4.B), which revealed a hydrophobic interaction, with the polar residues of the amphipathic presequence helix facing away from Tom20 (Abe et al., 2000; Saitoh et al., 2007). Based on this structural information, photopeptides were designed in order to define presequence receptors and their respective presequence binding domains. To this end the rat pALDH was modified by introducing the photoactivatable crosslinker p-benzoyl-phenylalanine (BPA) on either side of the amphipathic helix, replacing either Leu19 or Ser16 in $\mathrm{pL}_{19} \mathrm{~B}$ and $\mathrm{pS}_{16} \mathrm{~B}$, respectively. The BPA can be activated by UV light $(320-360 \mathrm{~nm})$ generating a radical at the ketone carbon atom and subsequently, C-C bonds are formed from adjacent C-H bonds (Dormán and Prestwich, 2000). The distance of this reaction is in the range of 3.1-14 $\AA$ (Dormán and Prestwich, 2000; Wittelsberger et al., 2006). One major advantage of benzophenones compared to other crosslinking groups is that several excitation and relaxation cycles are possible when no crosslink partner is in close vicinity, increasing the yield of adducts. The C-terminus of the peptides was modified by introducing an Arg followed by an aminobiotinyl-lysine and a His6 tag, allowing affinity purification and detection as well as removal of the tag by trypsin digestion during sample preparation for mass spectrometry (Figure 9). These peptides were chemically synthesized and purified by RP-HPLC (see Table 10). 


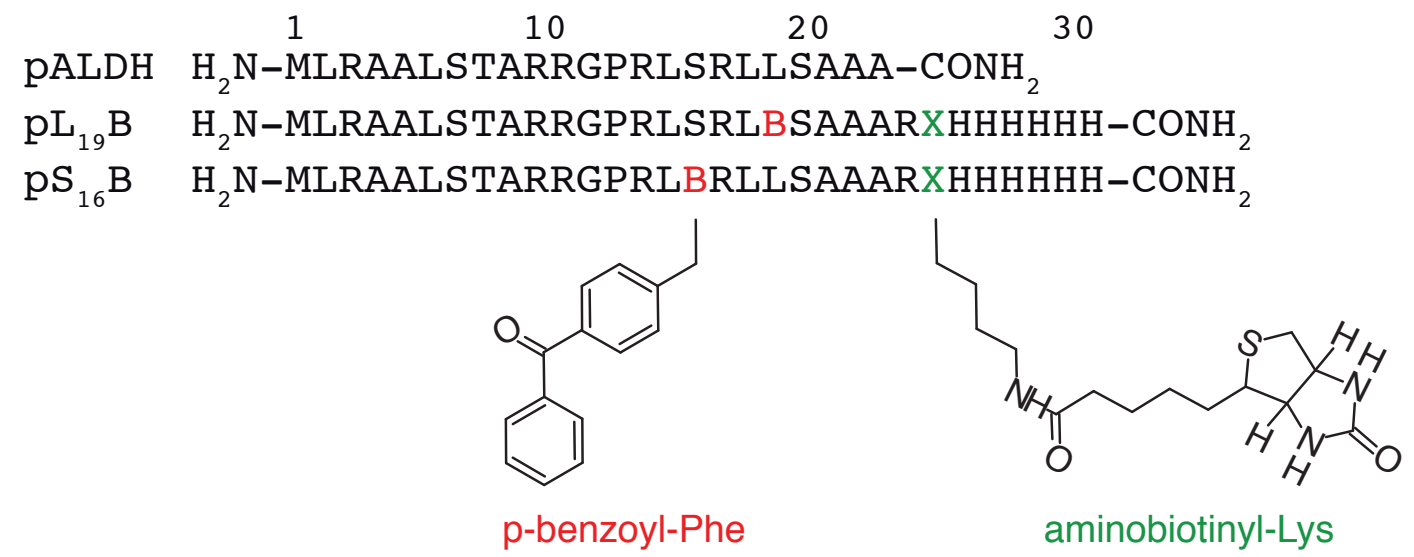

Fig. 9: Design of presequence photopeptides - The presequence photopeptides are based on the presequence of the rat aldehyde dehydrogenase (pALDH) with a parabenzoylpehnylalanine (B, BPA) moiety replacing Leu19 or Ser16 in $\mathrm{pL}_{19} \mathrm{~B}$ and $\mathrm{pS}_{16} \mathrm{~B}$, respectively. Furthermore a biotinyl lysine (X) and a hexa histidine tag were added to the $\mathrm{COOH}_{2}$ terminus. The chemical structures of the residues of BPA and biotinyl lysine are shown.

\subsubsection{Photopeptides are specific for the presequence import pathway}

The introduction of the BPA as well as the biotinyl-lysine added significant hydrophobic and bulky residues to the presequence peptides with the risk that this interferes with recognition and/ or translocation. Hence in vitro import of $\mathrm{pL}_{19} \mathrm{~B}$ and $\mathrm{pS}_{16} \mathrm{~B}$ into isolated yeast mitochondria was performed. Rapid, largely membrane potential dependent import of both peptides was observed already within $30 \mathrm{~s}$ (Figure 10.A), indicating transport into the matrix. In order to further determine the mitochondrial localization, the peptides were first imported and the mitochondria subsequently converted to mitoplasts (ruptured outer mitochondrial membrane) using a hypotonic buffer (Figure 10.B). Mitochondria were efficiently converted to mitoplasts with the inner membrane remaining intact, as judged by the accessibility of the IMS exposed inner membrane protein Tim50 to proteinase $\mathrm{K}$ and the PK protected matrix exposed inner membrane protein Pam17. A large fraction of the imported peptides remained protease protected under these conditions (lanes 4 and 5), while they were digested in solubilized mitochondria (lane 6 and 7). Hence, the photopeptides $\mathrm{pL}_{19} \mathrm{~B}$ and $\mathrm{pS}_{16} \mathrm{~B}$ are imported into the mitochondrial matrix as it was reported before for radiolabeled presequence peptides (Ono and Tuboi, 1988).

In order to validate that the presequence probes specifically follow the presequence pathway on their way to the mitochondrial matrix a competition experiment was per- 
A

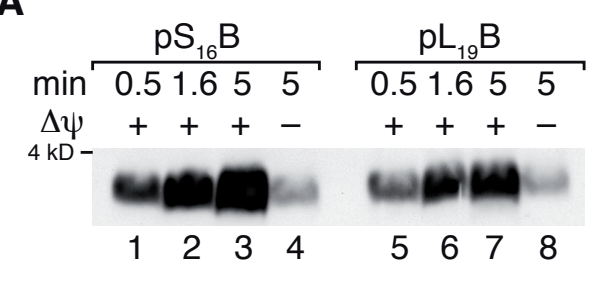

B

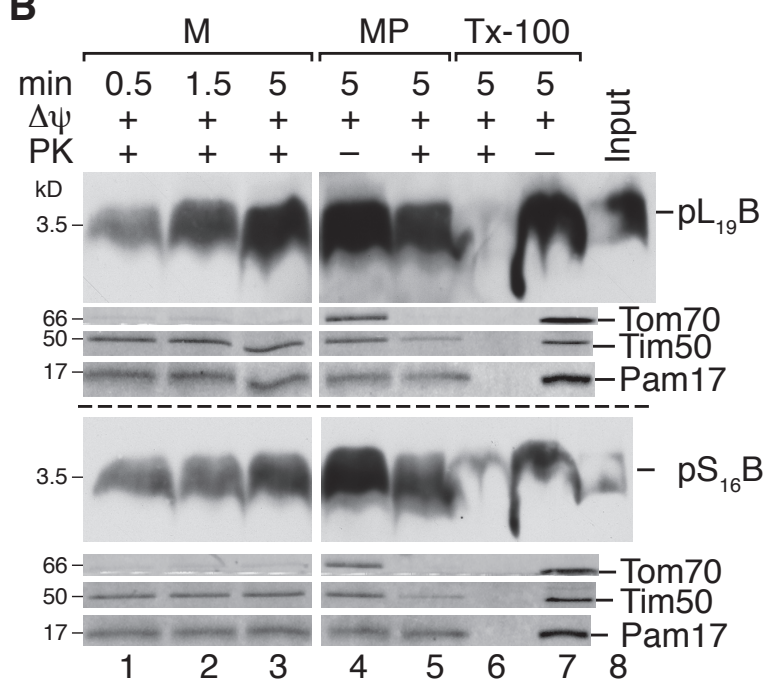

Fig. 10: Photopeptides import into mitochondria - A Photopeptides $\mathrm{pL}_{19} \mathrm{~B}$ and $\mathrm{pS}_{16} \mathrm{~B}$ were imported into isolated mitochondria at $25^{\circ} \mathrm{C}$ for the indicated time. The reaction was stopped by the addition of AVO mix and PK treatment. Samples were analyzed by SDS-PAGE and western blotting using SA-HRP. B After import of the photopeptides for the indicated times the reaction was stopped by the addition of AVO mix and mitochondria (M) were converted to mitoplasts (MP), or solubilized with Triton X100 (Tx-100) followed by PK treatment. Input shows $25 \%$ of the peptide used for each reaction. The samples were analyzed by western blot using the indicated antibodies or SA-HRP.

formed. The matrix targeted precursor $\mathrm{b}_{2}(167)_{\Delta}$-DHFR, a fusion protein consisting of the N-terminal part of cytochrome b (1-167, with a deletion of residues 47-65) fused to mouse dihydrofolate reductase (DHFR), was imported in the presence of different peptides (Figure 11). Successful import of the substrate was monitored by processing to the intermediate form (cleavage of the presequence by MPP) and PK protection. Reactions containing no peptide, or the respective amount of solvent resulted in similar import efficiencies. Competition with the authentic presequences of $\mathrm{Cox} 4$ (pCox4) and ALDH showed a significantly reduced import in the presence of $0.75 \mu \mathrm{M}$ peptide. In contrast the control peptides for both presequences, SynB2 (Allison and Schatz, 1986) and pALDH-s (Schulz et al., 2011), did not influence the import, even at six times higher concentrations. The photopeptides $\mathrm{pL}_{19} \mathrm{~B}$ and $\mathrm{pS}_{16} \mathrm{~B}$ inhibited $\mathrm{b}_{2}(167)_{\Delta}$-DHFR import at similar concentrations as the authentic presequence peptides.

Despite competition, the reduced precursor import in the presence of peptides could 


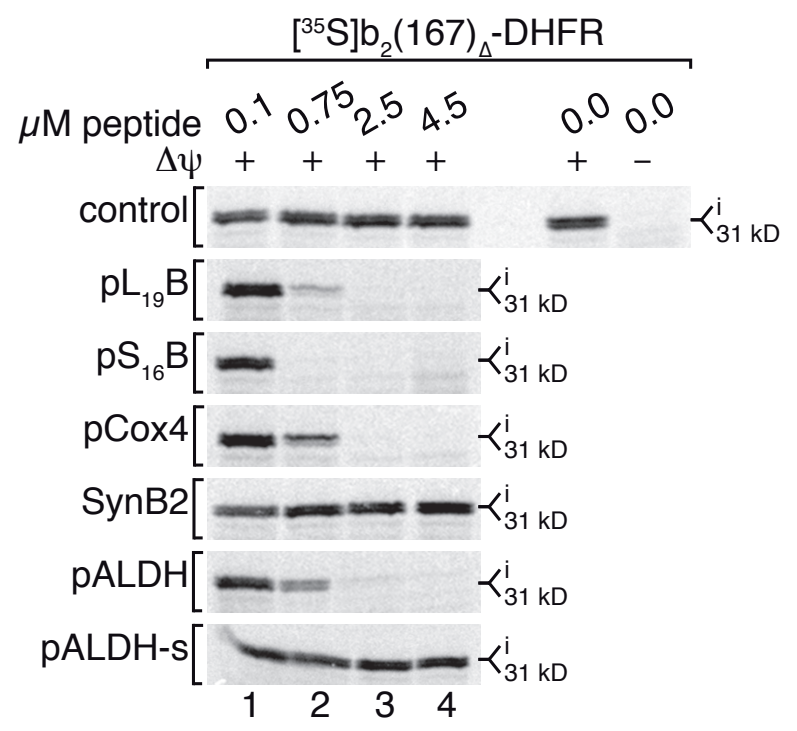

Fig. 11: Photopeptides inhibit presequence import - Import of $\left[{ }^{35} \mathrm{~S}\right] \mathrm{Met}$ labeled $\mathrm{b}_{2}(167)_{\Delta}$-DHFR in isolated mitochondria was performed for $15 \mathrm{~min}$ at $25^{\circ} \mathrm{C}$ in the presence of the indicated concentrations of the different peptides. Control samples contained equal volume of acetic acid. The reactions were stopped by the addition of AVO mix and PK treatment. Samples were analyzed by SDS-PAGE and autoradiography. i - intermediate.

also be explained by a damaging effect of the presequence peptides on the mitochondrial membranes. Large disruptions can be excluded as peptide import did not cause PK accessibility to the IMS and matrix (Figure 10.B lane 3 and 5). However, the complete integrity of the inner mitochondrial membrane is essential for maintenance of the membrane potential $(\Delta \psi)$. To exclude that the inner membrane was perforated, mitochondria were treated with pALDH, reisolated and then used for import of $b_{2}(167)_{\Delta}$-DHFR (Figure 12.A). The import efficiency of solvent and peptide treated mitochondria was similar (lane 4 and 6) indicating that the integrity of the mitochondrial membranes was unaffected. The incorporation of the BPA did not alter the effect of the peptide on the membrane, as also the removal of $\mathrm{pL}_{19} \mathrm{~B}$ restored import into the matrix (Figure 12.B). This was also supported by the observation that $2 \mu \mathrm{M}$ of presequence peptides and photopeptides did not inhibit import and assembly of the ADP-ATP-carrier (AAC), which depends on the membrane potential but is translocated by the TIM22 complex (Schulz et al., 2011). These results are in line with previous reports using similar concentrations of Cox 4 presequence peptides to inhibit matrix import (Glaser and Cumsky, 1990). 
A

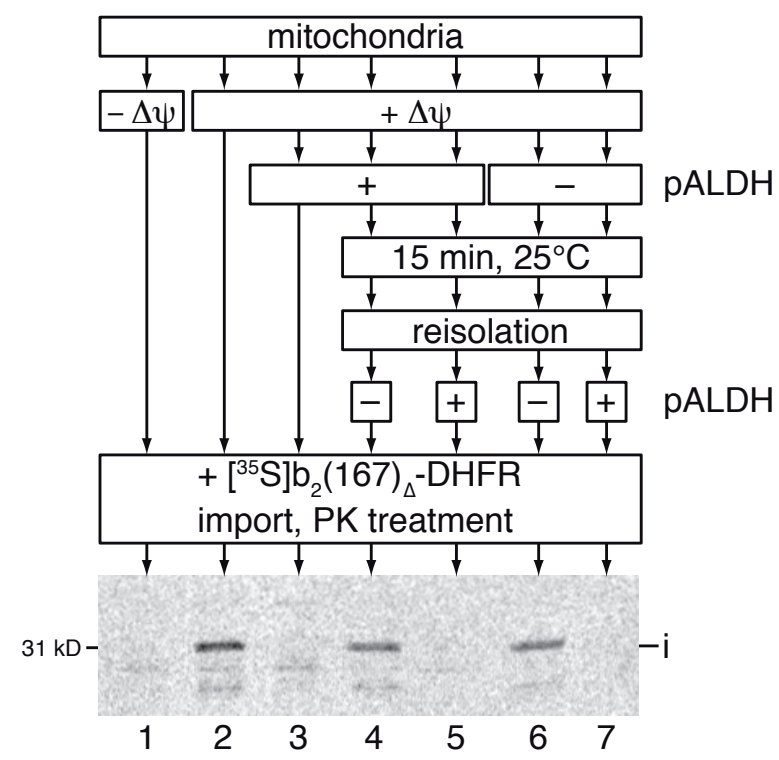

B

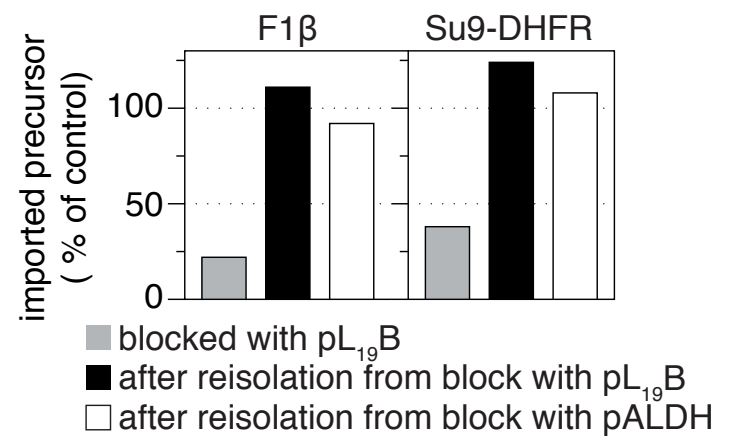

Fig. 12: Import inhibition by presequence peptides in reversible - A Radiolabeled $\mathrm{b}_{2}(167)_{\Delta}$-DHFR was imported into isolated mitochondria pretreated twice with or without $5 \mu \mathrm{M}$ pALDH for $15 \mathrm{~min}$ at $25^{\circ} \mathrm{C}$. The reaction was stopped by the addition of AVO mix and PK treatment. Samples were analyzed by SDS-PAGE and autoradiography. B Isolated mitochondria were incubated for $10 \mathrm{~min}$ at $25^{\circ} \mathrm{C}$ with $2 \mu \mathrm{M}$ of the indicated peptides. Following reisolation the import of F1 $\beta$ and Su9-DHFR for $15 \mathrm{~min}$ was assessed. The reactions were stopped by AVO mix and subsequent PK treatment. Samples were analyzed by SDS-PAGE and autoradiography. Imported precursor was quantified as percent of the respective precursor in mitochondria not treated with peptide $(100 \%)$. Grey bars represent the inhibition by $\mathrm{pL}_{19} \mathrm{~B}$, black and white bars the recovery after removal of $\mathrm{pL}_{19} \mathrm{~B}$ and $\mathrm{pALDH}$, respectively. $\mathrm{i}$ - intermediate.

\subsubsection{Identification of Tim50 as a novel presequence receptor}

As the newly developed photopeptides were efficiently transported along the authentic presequence pathway into the mitochondrial matrix, the photoactivatable crosslinker BPA can be used to trap interactions of the photopeptides with their cognate receptors in transit. To this end, photocrosslinking was performed in isolated mitochondria on ice. Tom20, whose presequence binding was the basis for the design of the peptides, showed a peptide- and UV light-dependent photoadduct, indicating successful crosslinking (Figure 13.A and C). This was also true for Tim50, a subunit of the presequence translocase in the inner mitochondrial membrane. For proteins not involved in presequence import 
like outer membrane Por1, the inner membrane protein Atp20 and the matrix proteins Mdj1 and Aco1 no adducts were detected. The same was true for some proteins of the import machinery like Pam17, Tim44, Tim17 and Hsp70 (Figure 13). Presequence photocrosslinks were also detected to the pore forming subunits Tom40 and Tim23, the internal targeting signal receptor Tom70 and to Tim21.

A

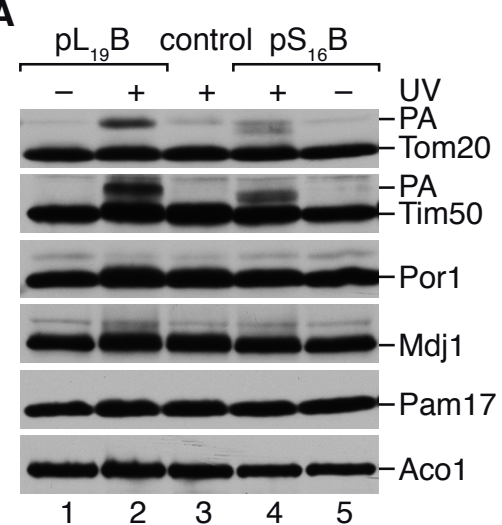

B

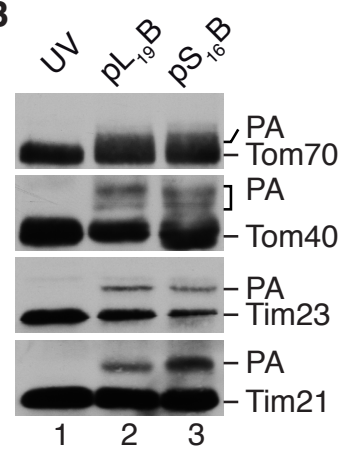

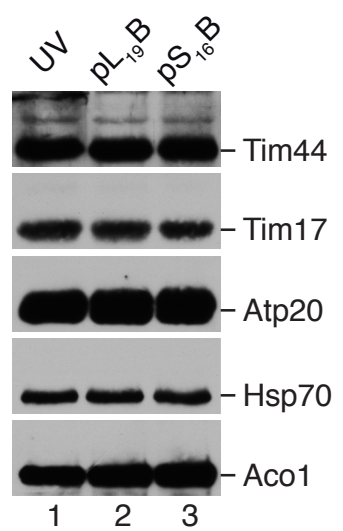

Fig. 13: Photopeptides crosslink to presequence receptors in organello - A Isolated mitochondria were incubated with $2 \mu \mathrm{M} \mathrm{pL}_{19} \mathrm{~B}, \mathrm{pS}_{16} \mathrm{~B}$ or the respective volume of acetic acid for $10 \mathrm{~min}$ on ice before subjecting the samples to UV irradiation for $30 \mathrm{~min}$ on ice. Samples were analyzed by SDS-PAGE and western blotting. PA - photoadduct. B Isolated mitochondria were photocrosslinked with $\mathrm{pL}_{19} \mathrm{~B}$ and $\mathrm{pS}_{16} \mathrm{~B}$ and analyzed as described in A. PA - photoadduct.

The biotin tag at the C-terminus of the photopeptides allowed isolation of photoadducts via denaturing streptavidin affinity chromatography (Figure 14.A). In addition to the adducts observed before for Tom20, Tom70 and Tim50, now also adducts to Tom22 were seen. Additionally, the slower migrating form of Tim50 was confirmed to be a photoadduct by immunoprecipitation of Tim50 and detection of the adduct using the biotin tag (Figure 14.B). 
A

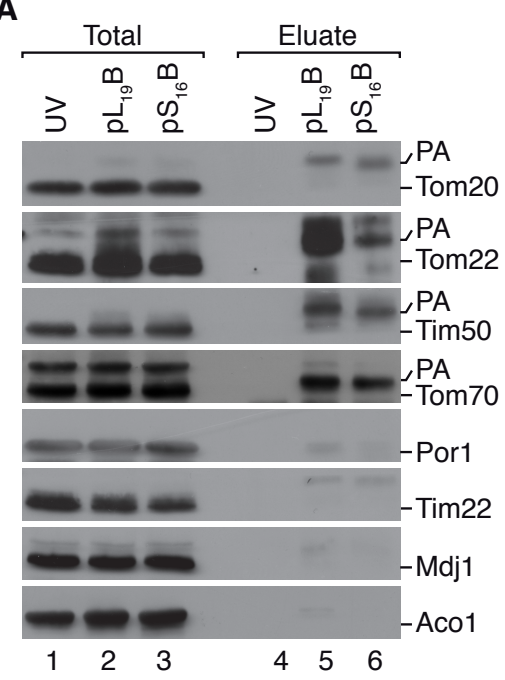

B

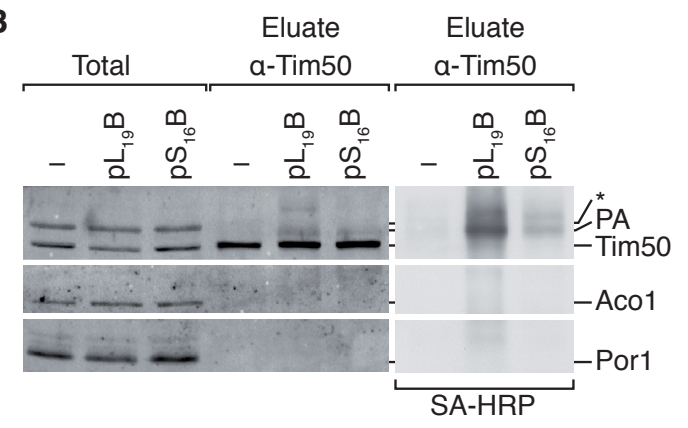

Fig. 14: Isolation of photoadducts to presequence receptors - A In organello photo cross-linking was carried out as in A. Subsequently, photoadducts were isolated using Streptavdin agarose and bound proteins were eluted with protein loading dye. Total represents $10 \%$ of the input. Samples were analyzed as in A. Total 5\%, eluate $100 \%$. B After in organello photo cross-linking as in A, digitonin solubilization and co-immunoprecipitation using $\alpha$-Tim50 antiserum were carried out. Samples were analyzed as in A. Total $5 \%$, eluate $100 \%$.

\subsubsection{Identification of a presequence binding domain in Tim50}

\subsubsection{Tim50 contains a C-terminal presequence binding domain}

Tim50 is an integral membrane protein and part of TIM23 ${ }^{\text {CORE }}$ (Geissler et al., 2002; Yamamoto et al., 2002; Mokranjac et al., 2003a). The protein is targeted to the inner membrane by a presequence and a sorting signal, hence making it a TIM23 substrate itself. A small domain without an assigned function is exposed to the matrix after cleavage of the presequence. In contrast the $45 \mathrm{kDa}$ IMS domain is known to interact with Tim23 ${ }^{\text {IMS }}$ (Yamamoto et al., 2002; Geissler et al., 2002; Tamura et al., 2009), contacts precursor proteins and transfers them from TOM to TIM23 (Yamamoto et al., 2002; Geissler et al., 2002; Mokranjac et al., 2003a) and is involved in the closure of the Tim23 channel (Meinecke et al., 2006; Alder et al., 2008b). The IMS domain contains a phosphatase like domain (NIF) as well as a C-terminal domain of so far undefined function (Figure 15.A).

The identification of photocrosslinks between the photopeptides and Tim50 indicated that it could be a presequence receptor of the TIM23 complex. In order to define the 
region of Tim50 interacting with the photopeptides in more detail, recombinant Tim50 ${ }^{\mathrm{IMS}}$ was purified and subjected to photocrosslinking (Figure 15.B). Crosslinking adducts were observed with both photopeptides (lane 2 and 3) and subsequent MS/MS analysis revealed that both peptides were crosslinked to Met412 in Tim50 (Figure 15.C and D). Based on secondary structure predictions the presequence binding domain (PBD) was defined as the C-terminal segment (residues 395-476) containing two predicted $\alpha$-helices connected by a linker (Figure 15.A and E).

The domain structure as well as the presequence binding domain are conserved in fungi (Figure 16.A). The domains, including the complete IMS domain of Tim50, one lacking the PBD (Tim50 $\triangle \mathrm{PBD}$ ) and the PBD alone, were expressed and purified from E. coli (Figure 16.B). Interestingly, a degradation product of similar size was observed in the Tim50 IMS purification that included the C-terminal domain as judged by mass spectrometry, suggesting that the PBD is stably folded and connected by a loose linker to the NIF domain.

All three domains were subjected to photocrosslinking using $\mathrm{pL}_{19} \mathrm{~B}$ and $\mathrm{pS}_{16} \mathrm{~B}$. Strong photoadducts were observed for the IMS domain as well as the PBD alone. However, deletion of the PBD abolished the formation of photoadducts (Figure 16.C). A similar result was obtained when chemically crosslinking SynB2 or pCox4 to Tim50 domains using DFDNB. Only the authentic presequence peptide pCox 4 formed adducts with Tim50 IMS and Tim50 PBD (Schulz et al., 2011). With both approaches a very weak adduct between Tim50 ${ }^{\triangle \mathrm{PBD}}$ and the presequence peptides was detected, indicating the possibility of a second binding site in Tim50. When Tim50 ${ }^{\mathrm{IMS}}$ was crosslinked to peptides in the presence of increasing salt concentrations the adduct formation to the authentic presequence peptide was not affected and no adduct was formed with the control peptides SynB2 (Figure 16.D).

When Tim50 ${ }^{\mathrm{PBD}}$ was photocrosslinked to the photopeptides several distinct photoadduct bands were observed, similar to the adducts with Tim50 ${ }^{\mathrm{IMS}}$ (Figure 15.B, 16.C and 17.A). The smaller size of the PBD allowed direct analysis by linear MALDI-TOF-MS in order to determine whether the adducts represent different crosslink configurations or a higher stoichiometry (Figure 17.B). The control sample clearly showed only one peak corresponding to the Tim50 $\mathrm{PBD}$. The samples photocrosslinked to $\mathrm{pL}_{19} \mathrm{~B}$ or $\mathrm{pS}_{16} \mathrm{~B}$ showed two additional peaks corresponding to Tim $50^{\mathrm{PBD}}+$ one or two peptides, indicating that in principle a 1:2 stoichiometry is possible, however the $1: 1$ ratio is 7 to 12.7 times more 

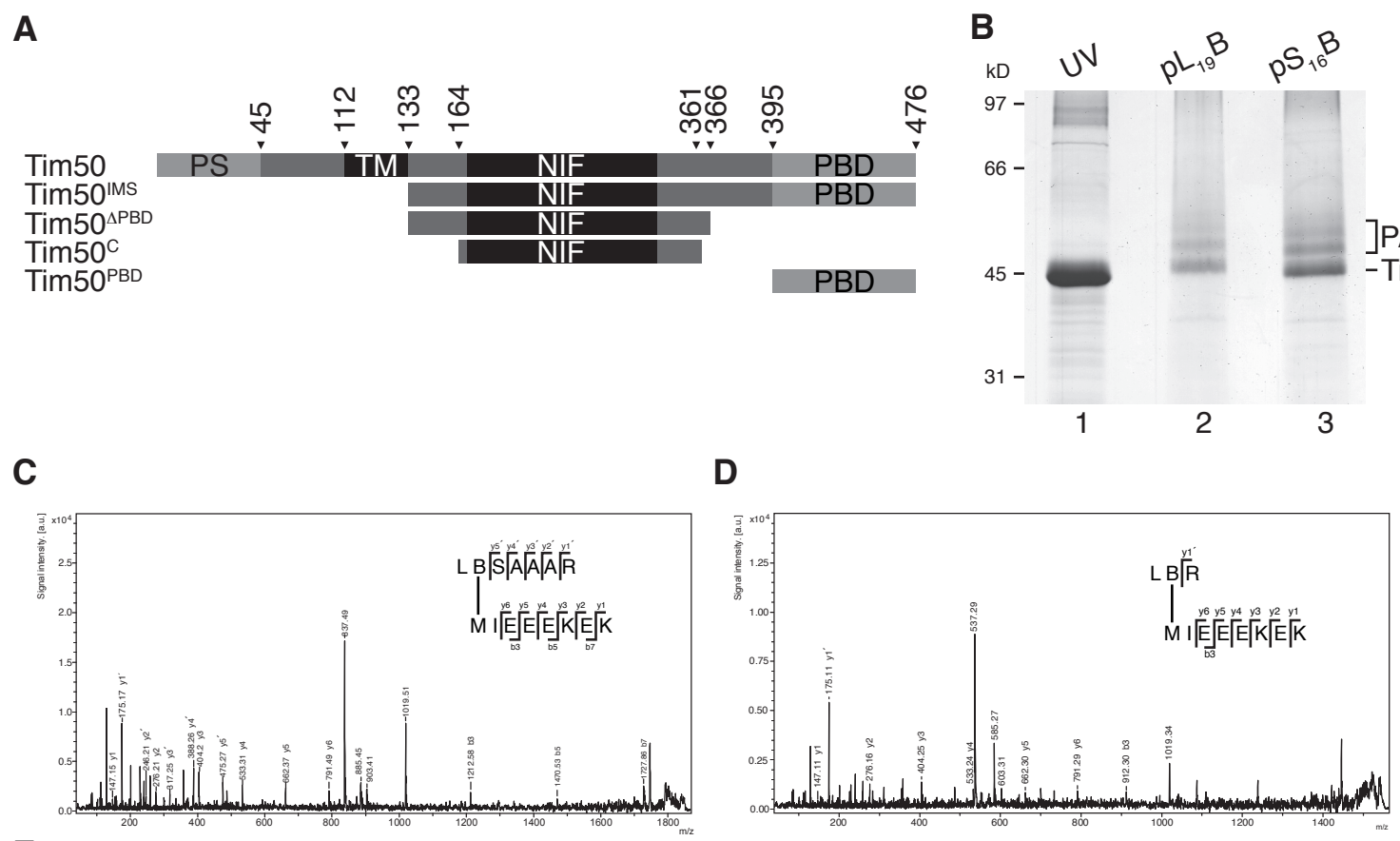

E

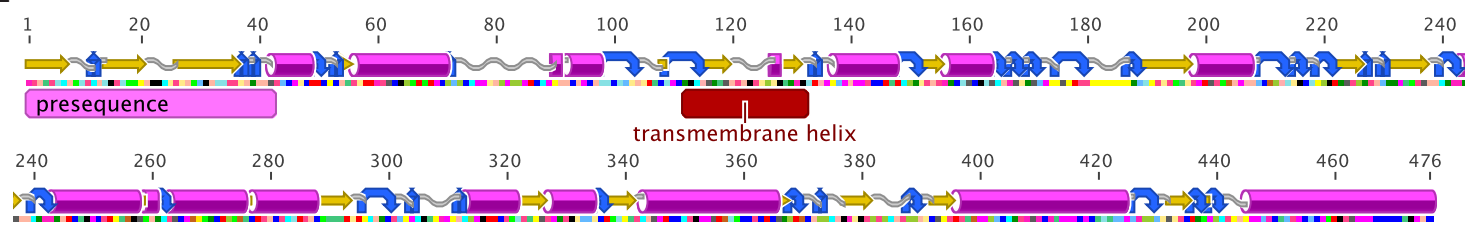

Fig. 15: Tim50 domains and identification of presequence binding domain - A Domains of Tim50 and the different constructs used in this study. PS - presequence, TM - transmembrane domain, NIF - NLI interacting factor-like phosphatase domain, PBD - presequence binding domain. B Purified Tim50 ${ }^{\mathrm{IMS}}$ was subjected to in vitro photocrosslinking for $30 \mathrm{~min}$. Samples were analyzed by SDS-PAGE and colloidal Coomassie staining. C Photoadduct of Tim $50^{\mathrm{IMS}}$ with $\mathrm{pL}_{19} \mathrm{~B}(\mathrm{~B}$, lane 2 ) was subjected to in-gel digestion with trypsin and subsequent LC-MALDI-MS/MS analysis. The $\mathrm{y}$ - and b-ion series of Tim50 ${ }^{412-419}$ crosslinked to $\mathrm{pL}_{19} \mathrm{~B}^{18-24}$ (parent ion mass: 1873.90) are indicated. D Photoadduct of Tim50 ${ }^{\mathrm{IMS}}$ with $\mathrm{pS}_{16} \mathrm{~B}(\mathrm{~B}$, lane 3 ) was analyzed as in C. The spectrum of Tim50 ${ }^{41-419}$ crosslinked to $\mathrm{pS}_{16} \mathrm{~B}^{15-17}$ is shown (parent ion mass: 1573.78). MALDI-MS-MS was performed by Dr. B. Schmidt (Institute of Cellular Biochemistry, Göttingen, Germany). E Secondary structure prediction of Tim50 using Geneious 5.3.6 with the EMBOSS tool garnier. Colors underneath the prediction indicate the amino acids with black $=\mathrm{A}$, yellow $=\mathrm{C}$, red $=\mathrm{D}, \operatorname{pink}=\mathrm{E}$, gray $=\mathrm{F}$, pale dark green $=\mathrm{G}$, brown $=\mathrm{H}$, pale light green $=\mathrm{I}$, blue $=\mathrm{K}$, pale pink $=\mathrm{L}$, magenta $=\mathrm{M}$, cyan $=\mathrm{N}$, bright yellow $=\mathrm{P}$, light blue $=\mathrm{Q}$, light cyan $=\mathrm{R}$, skin color $=\mathrm{S}$ and $\mathrm{W}$, pale yellow $=\mathrm{T}$, bright green $=\mathrm{V}$, dark green $=\mathrm{Y}$. 
A

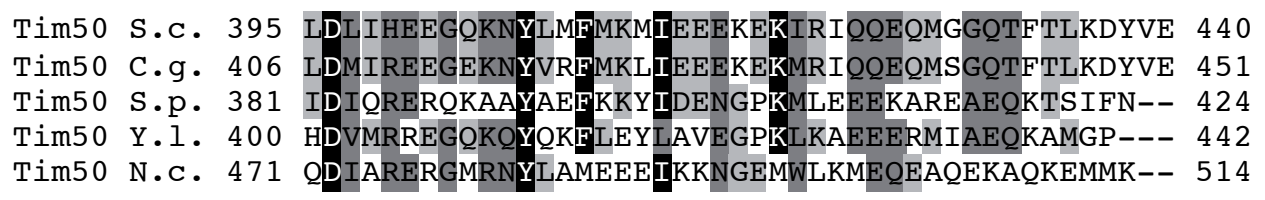

B

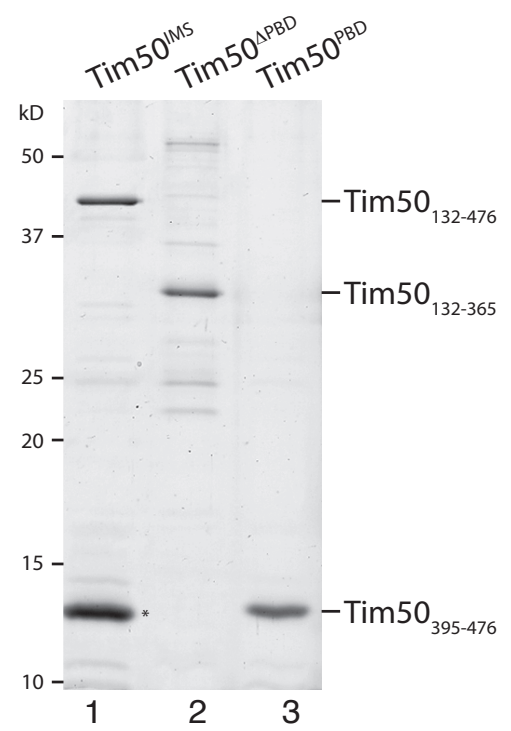

C

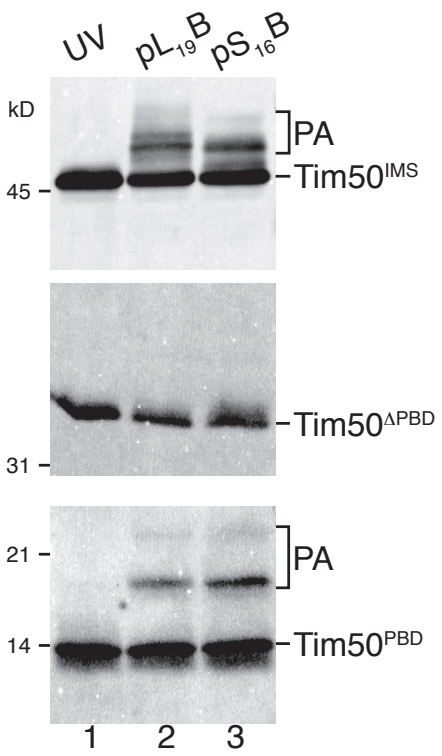

D

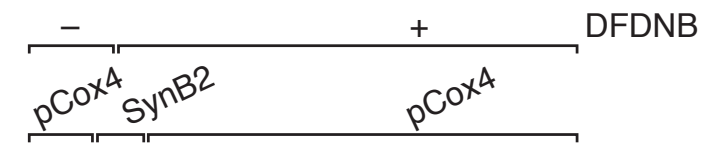

$\begin{array}{llllll}0 & 0 & 50 & 75 & 100,500_{2} 50_{5} 00 & \mathrm{mM} \mathrm{NaCl}\end{array}$

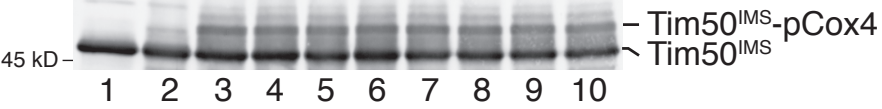

Fig. 16: Deletion of the presequence binding domain abolishes presequence crosslinking to Tim50 - A Alignment of the Tim50 ${ }^{\mathrm{PBD}}$ using ClustalW 2.0.11. Similarity rules according to Erdmann et al. (1991) $(\mathrm{G}=\mathrm{A}=\mathrm{S}, \mathrm{A}=\mathrm{V}, \mathrm{V}=\mathrm{I}=\mathrm{L}=\mathrm{M}$, $\mathrm{I}=\mathrm{L}=\mathrm{M}=\mathrm{F}=\mathrm{Y}=\mathrm{W}, \mathrm{K}=\mathrm{R}=\mathrm{H}, \mathrm{D}=\mathrm{E}=\mathrm{Q}=\mathrm{N}, \mathrm{S}=\mathrm{T}=\mathrm{Q}=\mathrm{N})$. Black: identical residues in at least four species, dark gray: similar residues in at least four species, light gray: similar residues in at least three species. S.c.: Saccharomyces cerevisiae, C.g.: Candida glabrata, S.p.: Schizosaccharomyces pombe, Y.l.: Yarrowia lipolytica, N.c.: Neurospora crassa. B Purified Tim50 domains as described in Figure 15.A. * - stable degradation product tht corresponds to the PBD. C Photocrosslinking of purified Tim50 domains with $\mathrm{pL}_{19} \mathrm{~B}$ and $\mathrm{pS}_{16} \mathrm{~B}$. Samples were analyzed by SDS-PAGE and western blotting. D $1 \mu \mathrm{M}$ Tim50 ${ }^{\mathrm{IMS}}$ was chemically crosslinking using $100 \mu \mathrm{M}$ DFDNB for $30 \mathrm{~min}$ on ice to $5 \mu \mathrm{M}$ pCox 4 or SynB2 in the presence of increasing salt concentrations. Samples were analyzed as in $\mathrm{C}$. 
abundant. Hence, the almost equal amounts of the two photoadduct bands seen on the western blot represents to a large extent different configurations of crosslinks and less than $10 \%$ of the adducts represent a 1:2 stoichiometry. Similar observations have been made before (Junge et al., 2004; Alder et al., 2008b). Additional crosslinks to Met410, 412, 427 and 449 were observed and might explain the different configurations (Schulz et al., 2011).

\subsubsection{Attempts to obtain a high resolution structure of Tim50 ${ }^{\mathrm{PBD}}$}

Structural insight into the presequence recognition would be possible with a structure that contains the complete IMS domain or the PBD alone. The attempt by Qian et al. (2011) to solve the IMS structure led to a structure of a protease resistant fragment that corresponds to the NIF domain, therefore lacking the PBD (Figure 6.B).

To overcome this, the PBD was purified from E. coli in mg scale (Figure 18). IPTG induced expression for $4 \mathrm{~h}$ at $37^{\circ} \mathrm{C}$ generated a soluble protein that was purified via IMAC (Figure 18.A). The major fractions containing the protein were cleaved with His-tagged TEV protease to remove the $6 \mathrm{x}$ His tag (Figure 18.B). After removal of the tag and protease the protein was relatively pure (lane 3 ). In order to validate that a soluble protein and no aggregates are used, it was further purified by gel filtration resulting in one major peak containing Tim50 $\mathrm{PBD}$ (Figure 18.C). The elution volume indicates that the PBD forms a trimer. Crystallization trails using different conditions were performed, however so far only spherulites or very small crystals were obtained (Figure 18.D). Hence, optimization of the crystallization conditions is still ongoing.

\subsubsection{Tim50 contains a second presequence binding site}

When the crosslinking of photopeptides and pCox 4 to Tim50 ${ }^{\triangle \mathrm{PBD}}$ was analyzed a faint adduct was observed (Figure 16.C lane 2). However, this construct showed low solubility and stability compared to the other Tim50 domains used. The crystallized fragment $\left(\operatorname{Tim} 50^{\mathrm{C}}\right)$ has a shorter N-terminus and a slightly shorter C-terminus compared to the previously used construct (residues 133 to 366 compared to 164 to 361) (Figure 15.A). Hence, the ability to generate photoadducts with the Tim $50^{\mathrm{C}}$ construct was analyzed (Figure 19.A). Tim50 ${ }^{\mathrm{IMS}}$ showed a clear photoadduct formation that was visible when detecting with both the $\alpha$-Tim50 antibody and the Streptavidin conjugate, while the 
A

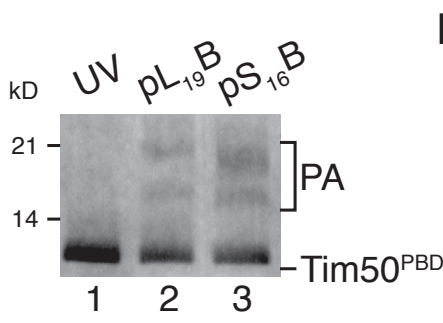

B

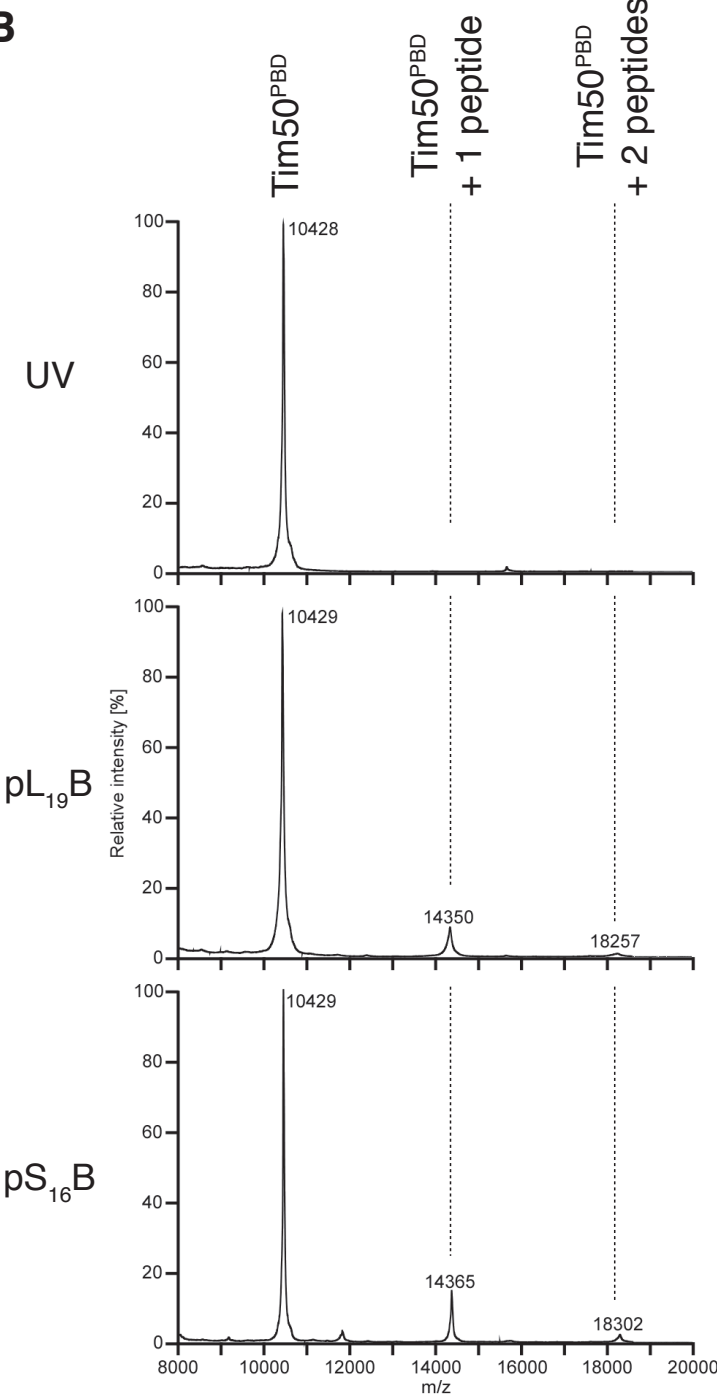

Fig. 17: Tim50 ${ }^{\mathrm{PBD}}$ photoadducts contain one peptide in different configurations A Purified Tim50 ${ }^{\mathrm{PBD}}$ was subjected to photocrosslinking. Samples were analyzed by SDS-PAGE and western blotting. B Linear MALDI-TOF-MS analysis of the samples shown in A. The theoretical monoisotopic masses are: Tim50 ${ }^{\mathrm{PBD}}$ : $10428 \mathrm{Da}$, $\operatorname{Tim} 50^{\mathrm{PBD}}+1 \mathrm{pL}_{19} \mathrm{~B}: 14338 \mathrm{Da}$, Tim50 ${ }^{\mathrm{PBD}}+2 \mathrm{pL}_{19} \mathrm{~B}: 18247 \mathrm{Da}, \operatorname{Tim} 50^{\mathrm{PBD}}+1 \mathrm{pS}_{16} \mathrm{~B}$ : $14364 \mathrm{Da}$, Tim50 ${ }^{\mathrm{PBD}}+2 \mathrm{pS}_{16} \mathrm{~B}: 18299 \mathrm{Da}$. Relative area under the curve for the indicated peaks are: $\mathrm{pL}_{19} \mathrm{~B}: 80.8,17.8$ and $1.4 \%, \mathrm{pS}_{16} \mathrm{~B}: 83.4,14.6$ and $2.0 \%$. Shifts to higher mass to charge ratios are most likely due to oxidation of Met. Linear MALDITOF-MS was performed by Dr. O. Jahn (MPI for experimental medicine, Göttingen, Germany). 
A
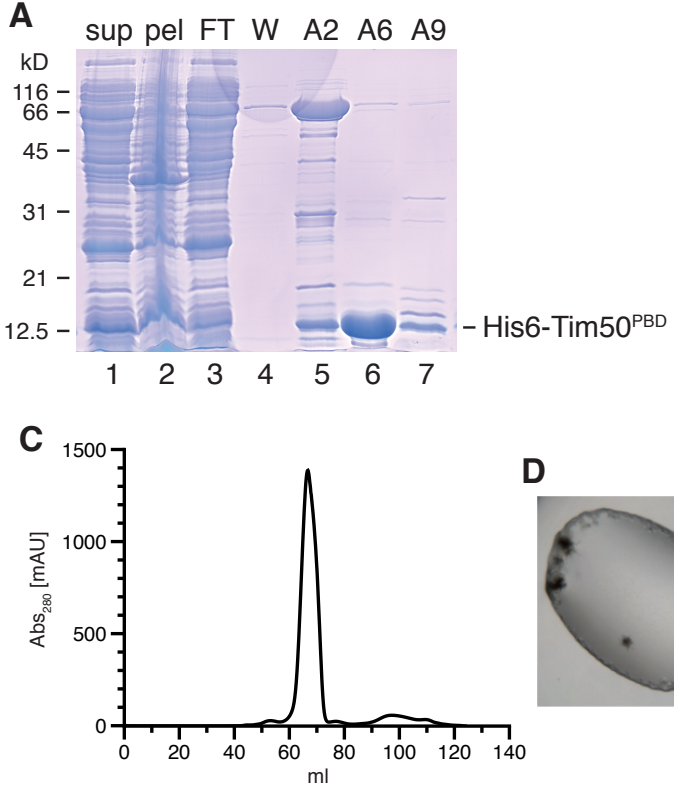

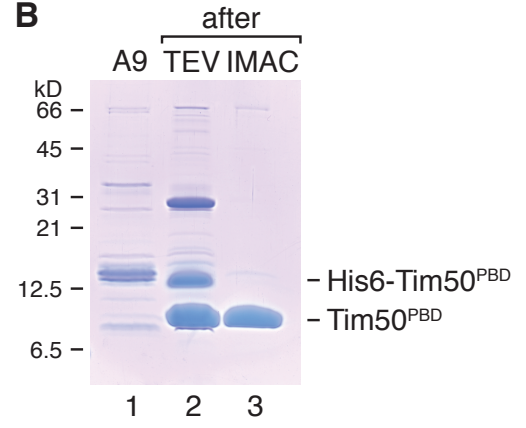

D
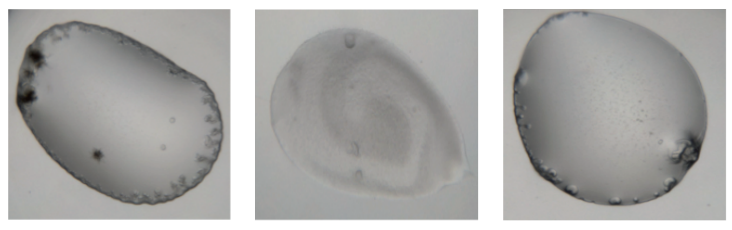

Fig. 18: Purification of Tim50 ${ }^{\mathrm{PBD}}$ and crystallization trials - A Purification His6Tim50 ${ }^{\mathrm{PBD}}$ by IMAC. Sup: supernatant, pel: pellet, FT: flow through, W: wash, A2-9: elution fraction. B Cleavage of the His6 tag using His-tagged TEV protease. Samples after cleavage for $2 \mathrm{~h}$ at $30^{\circ} \mathrm{C}$ using $0.06 \mathrm{mg} / \mathrm{mg}$ and subsequent removal of the protease and tag by IMAC are shown together with fraction A9 after the initial IMAC. C The cleaved Tim50 ${ }^{\mathrm{PBD}}$ (B, lane 3) was concentrated and loaded on a Superdex75 16/60 HiLoad column. The chromatogram shows the absorption at $280 \mathrm{~nm}$. The $10.5 \mathrm{kDa}$ PBD elutes at $68 \mathrm{~mL}$, corresponding to approximately $33 \mathrm{kDa}$. D Fractions of the major peak of the gel filtration were pooled, concentrated and subjected to crystallization trails. Preliminary results are shown, from left to right: $0.2 \mathrm{M} \mathrm{MgCl}_{2}$, $10 \%$ (w/v) polyethylene glycol 8000, 0.1 M Tris pH 7.0; $0.02 \mathrm{M} \mathrm{MgCl}, 22 \%$ (w/v) polyacrylic acid 5100 sodium salt, $0.1 \mathrm{M}$ Hepes pH 7.5; $0.05 \mathrm{M} \mathrm{MgCl}_{2}, 0.2 \mathrm{M} \mathrm{KCl}, 10 \%$ $(\mathrm{w} / \mathrm{v})$ polyethylene glycol 4000, $0.05 \mathrm{M}$ Tris- $\mathrm{HCl} \mathrm{pH}$ 7.5. Crystallization trails were carried out by Dr. A. Dickmanns, Dept. for Molecular Structural Biology, Göttingen, Germany. 
Tim50 ${ }^{\triangle \mathrm{PBD}}$ generated a very weak photoadduct which was only detected with SA-HRP. This adduct was more clearly defined with the Tim $50^{\mathrm{C}}$ construct and also detectable with the $\alpha$-Tim50 antibody. Hence, increased solubility and stability of the crystallized construct are beneficial for an increased photoadduct yield, however compared to the IMS domain the efficiency of adduct formation is very low.

In order to identify the region within the crystallized construct responsible for presequence binding Tim50 ${ }^{\mathrm{C}}$ purified protein was mixed 1:1 with $\mathrm{pL}_{19} \mathrm{~B}$ and $\mathrm{pS}_{16} \mathrm{~B}$ and photocrosslinking was performed (Figure 19.B). Western blot analysis using $\alpha$-Tim50 antibodies as well as streptavidin-HRP revealed photoadducts between Tim50 ${ }^{\mathrm{C}}$ and both peptides. Colloidal coomassie stained photoadducts were analyzed by MALDI-MS-MS and crosslinks in vicinity to the negatively charged groove were found (Figure 6.B).

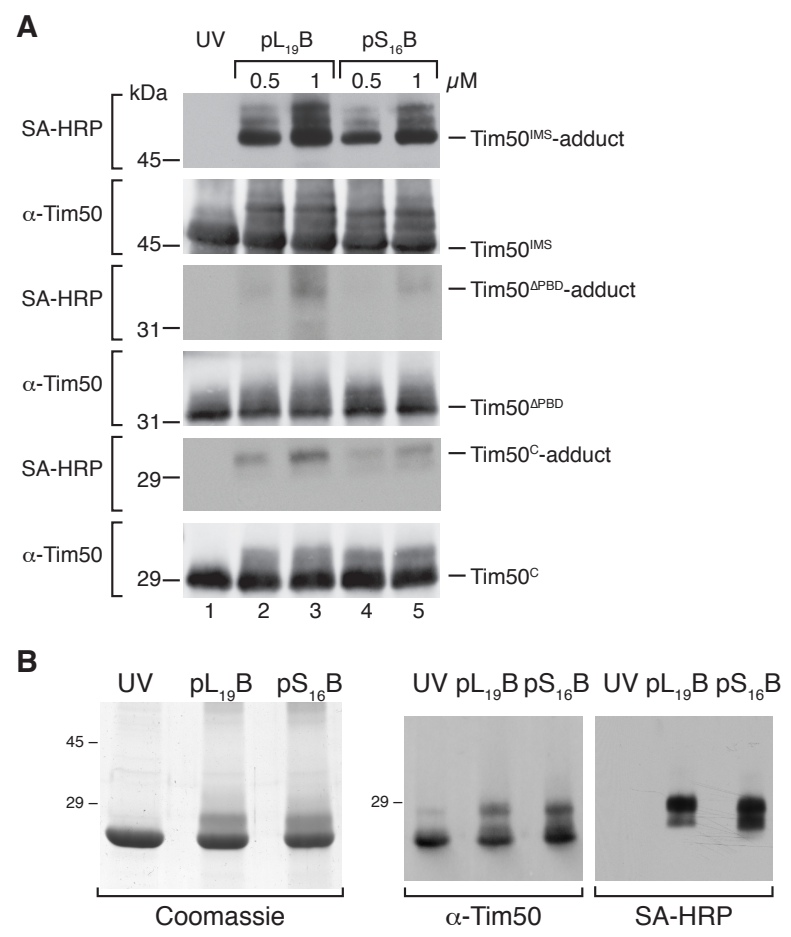

Fig. 19: $\operatorname{Tim}^{\mathrm{C}} \mathrm{C}^{\mathrm{C}}$ crosslinks more efficiently to presequences than Tim50 ${ }^{\Delta \mathrm{PBD}}$ - A $1 \mu \mathrm{M}$ purified Tim50 domains (IMS; $\triangle \mathrm{PBD}$ and the crystallized fragment $(\mathrm{C})$ were photocrosslinked with 0.5 and $1 \mu \mathrm{M} \mathrm{pL}_{19} \mathrm{~B}$ and $\mathrm{pS}_{16} \mathrm{~B}$. Samples were analyzed by SDSPAGE and western blotting using either Streptavidin-HRP (SA-HRP) or $\alpha$-Tim50 antibodies. B Purified TEV-cleaved Tim50 ${ }^{\mathrm{C}}$ was photocrosslinked with $\mathrm{pL}_{19} \mathrm{~B}$ and $\mathrm{pS}_{16} \mathrm{~B}$. Samples were analyzed by colloidal Coomassie staining or western blotting using the indicated antibodies/ conjugates. 
Crosslinking efficiencies are not necessarily representative for affinities due to their dependence on functional groups. Hence, SPR with immobilized Tim50 domains was performed. The affinities were calculated from the kinetic data obtained during the association and dissociation phases (Table 4). The presequence binding domain alone showed approximately two-fold lower affinity compared to the IMS domain. Surprisingly, the crystallized construct, lacking the PBD, showed almost no difference to the full IMS domain. This is in strong contrast to the crosslinking results (Figure 16.B and 19).

Hence in vitro the presequence binding domain located in the C-terminal domain of Tim50 ${ }^{\mathrm{IMS}}$ is sufficient but not necessary for presequence binding. A second binding site can be found in proximity to the negatively charged groove.

Tab. 4: Affinities of different Tim50 domains to presequence peptides - $K_{D}$ of immobilized Tim50 domains to pALDH as determined by kinetic SPR parameters. Mean \pm SEM are shown, $\mathrm{n}=4$. Carried out with the help of Dr. O. Lytovchenko (Institute of Cellular Biochemistry, Göttingen, Germany) and Markus Kilisch (Institute of Molecular Biology, Göttingen, Germany).

\begin{tabular}{lll}
\hline \hline Ligand & - analyte & $\mathrm{K}_{\mathrm{D}}[\mu \mathrm{M}]$ \\
\hline Tim50 & $-\mathrm{pALD}$ & $3.79 \pm 0.22$ \\
Tim50 & $-\mathrm{pALDH}$ & $6.94 \pm 1.18$ \\
Tim50 & $-\mathrm{pALDH}$ & $3.94 \pm 0.47$ \\
\hline \hline
\end{tabular}

\subsubsection{Characterization of Tim50's presequence binding domain}

\subsubsection{The presequence binding domain of Tim50 is essential for cell viability}

The identification of the presequence binding domain in Tim50 raised the question of its relevance in vivo, especially concerning the results showing that the crystallized construct, which lacks the PBD showed no decreased affinity towards presequences. To this end the diploid BY4743 yeast strain was transformed with a deletion module deleting

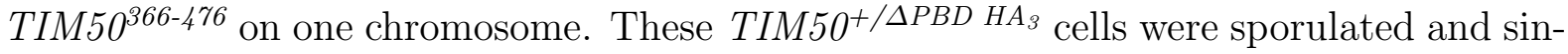
gle tetrads were separated (Figure 20.A). Following growth on full medium a 1:1 ratio was observed, with only two viable spores of each tetrad, indicating a lethal phenotype of the deletion. In a previous analysis it was found that a gene deletion, which was considered lethal by sporulation analysis, could be obtained when plasmid loss was used as an 
alternative strategy (Kovermann et al., 2002). Therefore, strains containing TIM50 on a URA3 plasmid complementing a chromosomal TIM50 deletion were transformed with plasmids encoding Tim50, Tim50 ${ }^{\triangle \mathrm{PBD}}$ or the HA tagged variants. When these cells were plated on 5-FOA containing medium they are selected for the loss of URA3 plasmid, exposing the phenotype of the proteins of interest. Both Tim50 and Tim50 ${ }^{\mathrm{HA}}$ complemented the deletion, while the empty plasmid, Tim50 $\triangle \mathrm{PBD}$ and Tim50 4 PBD HA did not complement (Figure 20.B and C), neither did the shorter Tim50 ${ }^{\mathrm{C}}$ construct (Figure 20.D). This indicated that the presequence binding domain is essential for cell viability irrespective of the putative presequence binding groove in the NIF domain. All further studies in yeast were therefore carried out with the $\triangle \mathrm{PBD}$ construct (amino acids 1-365). In mitochondria isolated from the diploid $T I M 50^{+/ \triangle P B D H A_{3}} \operatorname{Tim} 50^{\triangle \mathrm{PBD}}$ was stable at steady state as it was readily detectable (Figure 20.E). In organello photocrosslinking in these mitochondria revealed that the wild-type Tim50 generated photoadducts with $\mathrm{pL}_{19} \mathrm{~B}$ and $\mathrm{pS}_{16} \mathrm{~B}$ just like the mitochondria isolated from the diploid TIM50. In contrast, crosslinking efficiency of Tim $50^{\triangle \mathrm{PBD}}$ with the photopeptides was strongly decreased, similar to the results obtained in vitro.

Hence, despite the second presequence binding site in the NIF domain of Tim50, recognition by the $\mathrm{C}$-terminal $\mathrm{PBD}$ is essential for cell viability.

\subsubsection{The interaction between Tim50 and Tim23 is independent of the PBD}

The interaction of Tim50 and Tim23 is crucial for channel closure (Meinecke et al., 2006). Recently Qian et al. (2011) identified a $\beta$-loop in Tim50 ${ }^{\mathrm{IMS}}$ responsible for binding to Tim $23^{\text {IMS }}$. Therefore the ability of the truncated Tim50 construct to bind to Tim23 was analyzed using a pulldown assay with immobilized Tim $23^{\mathrm{IMS}}$. Tim50 ${ }^{\triangle \mathrm{PBD}}$ retained approximately $30 \%$ binding to Tim $23^{\text {IMS }}$ in solution compared to wild-type Tim50 (Figure 21.A).

In addition to the interaction of both IMS domains (Geissler et al., 2002; GevorkyanAirapetov et al., 2009), in mitochondria Tim50 also interacts with Tim23 by contacts of the transmembrane domains (Alder et al., 2008b). In order to compare the presence of either allele in the TIM23 complex under native conditions the yeast strain containing TIM50 under control of the GAL1 promoter (Geissler et al., 2002) was transformed with plasmids encoding for Tim $50^{\mathrm{HA}}$ or Tim $50^{\triangle \mathrm{PBD}-\mathrm{HA}}$. Mitochondria were isolated from these strains under conditions when the GAL1 promoter was repressed by glucose, hence 
A

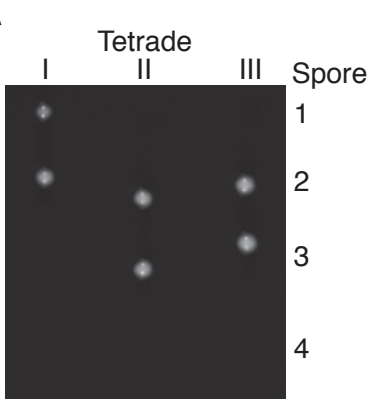

B
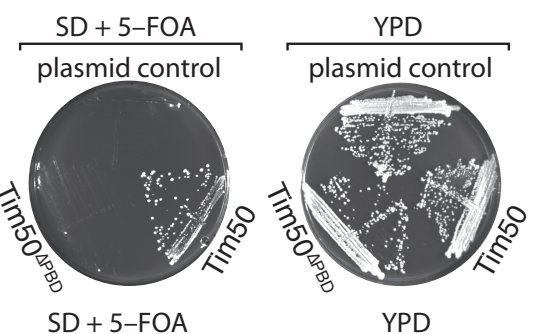

C
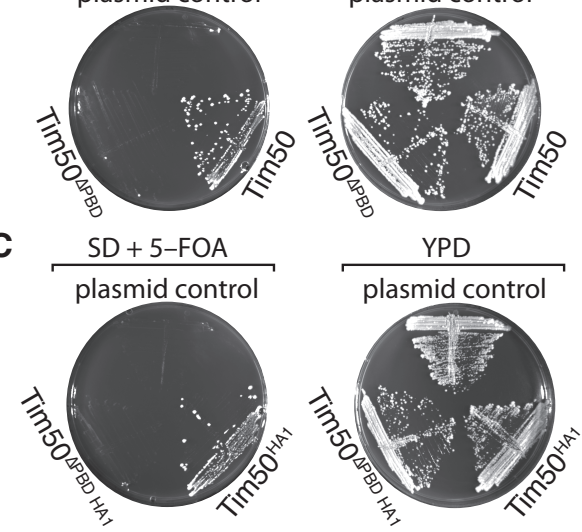

D
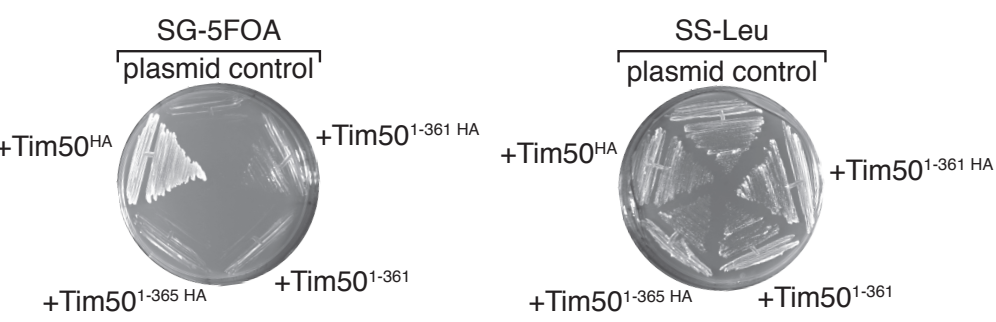

E

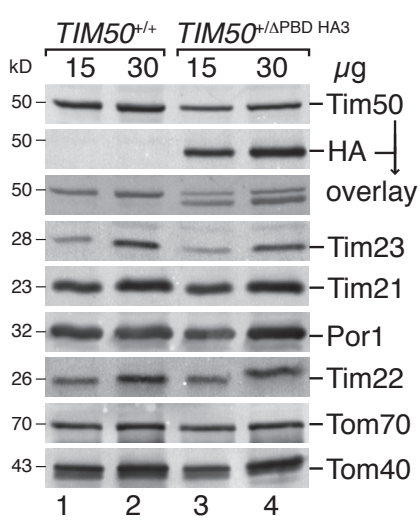

F

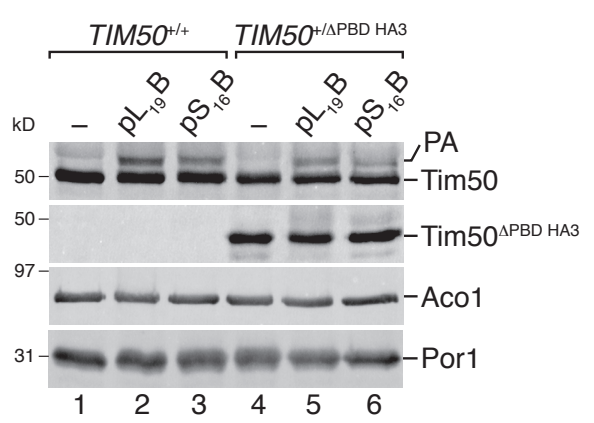

Fig. 20: Tim50 ${ }^{\text {PBD }}$ is essential for cell viability - A Diploid BY4743 yeast cells carrying a single chromosomal deletion of TIM50 $366-476$ were sporulated and tetrads were separated. Single spores were grown on YPD plates. B YPH499 yeast cells carrying a chromosomal deletion of TIM50 were complemented with a TIM50-containing URA3 plasmid and transformed with plasmids encoding Tim50 or Tim50 ${ }^{\Delta \mathrm{PBD}}$. The cells were subjected to plasmid loss on 5-FOA containing selective medium or streaked out on full medium. C As in B, except that cells were transformed with plasmids encoding $\operatorname{Tim} 50^{\mathrm{HA}}$ or Tim50 $\mathrm{PBBD}-\mathrm{HA}$. (Continued on next page.) 
Fig. 20: (Continued from previous page.) - D Cells as in B were transformed with plasmids encoding Tim50 ${ }^{\mathrm{HA}}$, Tim50 1-365 HA , Tim50 $0^{1-365}$ or Tim50 $0^{1-365 ~ H A}$ and subsequently subjected to plasmid loss on 5-FOA containing selective medium or streaked out on selective medium. E Protein levels in mitochondria isolated from the diploid TIM50 ${ }^{+/+}$ and $T I M 50^{+/ \triangle P B D ~} \mathrm{HA}_{3}$ analyzed by SDS-PAGE and western blotting with the indicated antibodies. F In organello photocrosslinking in the mitochondria described in E. Samples analyzed as in E.

generating a situation were the phenotype of Tim50 $\triangle \mathrm{PBD}-\mathrm{HA}$ can be investigated without interference of the genomically encoded wild-type copy (Figure 21.B). The truncated protein was expressed at slightly higher levels compared to the Tim50 ${ }^{\mathrm{HA}}$ variant and the shutdown of the chromosomal Tim50 reduced its levels drastically. Subsequently, co-immunoprecipitations were performed from these mitochondria using the C-terminal HA-tag and digitonin solubilization. With both Tim50 variants similar amounts of Tim23 and Tim17 co-precipitated (Figure 21.D) indicating the presence of both variants in the TIM23 complex. Hence, the Tim23 and presequence binding domain are separate in Tim50.

\subsubsection{The interaction between Tim50 and presequence peptides is indepen- dent of Tim23}

It was previously shown by Mokranjac et al. (2009) that the proximity of a substrate to Tim50 was dependent on Tim23. However, as Tim50 interacted with presequence peptides in vitro independent of Tim23 and its interaction with Tim23 was not altered by the deletion of the PBD in mitochondria, the dependence of the Tim50-presequence interaction on Tim23 in mitochondria was analyzed. To this end mitochondria overexpressing Tim50 were subjected to photocrosslinking with $\mathrm{pL}_{19} \mathrm{~B}$ and $\mathrm{pS}_{16} \mathrm{~B}$ (Figure 22.A). In the Tim50个 mitochondria the intensity of the presequence photoadduct increased in the same way the Tim50 signal increased due to the overexpression, indicating that Tim50 and no other subunit was the limiting factor. In line with the increased photoadduct, the import of the matrix targeted precursor F1 $\beta$ was more efficient in the Tim50^ mitochondria compared to the wild-type (Figure 22.B). To exclude that this is because of an increased association of Tim50 with Tim23, mitochondria with strongly reduced levels of Tim23 (Tim23 $\downarrow$ ) were prepared (Figure 22.C). A consequence of the dramatically reduced Tim23 levels was the reduction of TIM23 substrates like Aco1 and Pam17. F1ß import 

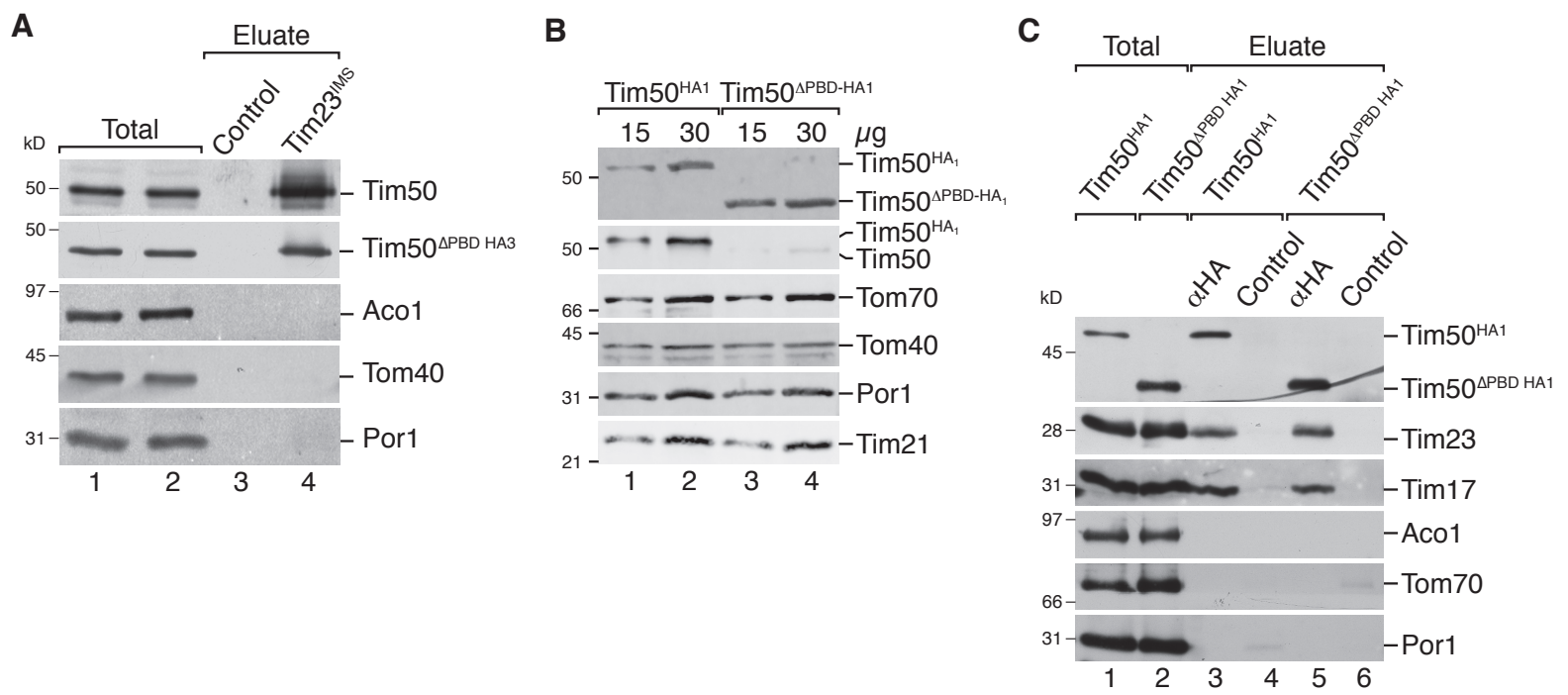

Fig. 21: The Tim50 presequence binding domain and Tim23 binding domain are separate. - A Purified His-tagged Tim $23^{\mathrm{IMS}}$ was immobilized on Ni-NTA agarose and incubated with mitochondrial detergent extract. Bound proteins were eluted and analyzed by SDS-PAGE and western blotting. Empty Ni-NTA agarose was used as a control. Total $5 \%$, eluate $100 \%$. B Mitochondria isolated from yeast strains expressing Tim50 ${ }^{\mathrm{HA} 1}$ or Tim50 ${ }^{\triangle \mathrm{PBD} H A 1}$ as well as reduced Tim50 levels. Samples were analyzed by SDS-PAGE and western blotting. C Co-immunoprecipitation of digitonin extracts from the mitochondria described in B using $\alpha-\mathrm{HA}$ or $\alpha-\mathrm{His}_{6}$ as a control. Samples were analyzed as in B. Total $5 \%$, eluate $100 \%$.

was strongly impaired in these mitochondria due to the lack of the translocation channel across the inner membrane (Figure 22.D). Photocrosslinking and chemical crosslinking in these mitochondria revealed that Tim50 could still interact with presequence peptides in the absence of Tim23 (Figure 22.E and F).

\subsubsection{Deletion of the PBD in Tim50 impairs presequence import across the inner membrane}

To investigate the effect of the selective deletion of the presequence binding domain of Tim50, Tim50 $\downarrow+\operatorname{Tim} 50^{\triangle \mathrm{PBD}-H A}$ mitochondria were used (Figure 21.B). Import of the matrix targeted precursors F1 3 and Su9-DHFR was reduced to approximately $25 \%$ of the wild-type (Figure 23.A and B). Despite its function in the TIM23 complex, Tim50 was also suggested to be involved in the precursor transfer from the TOM to the TIM23 complex acting before the IMS domain of Tom22 (Chacinska et al., 2005; Mokranjac 
A

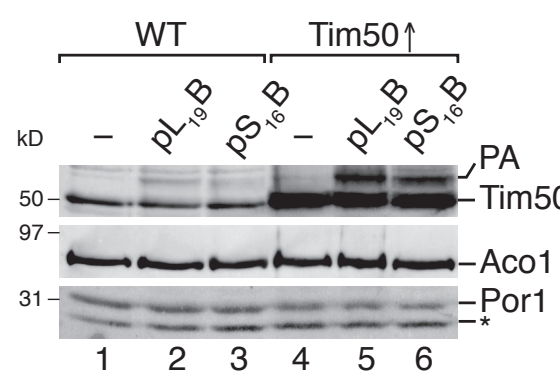

C

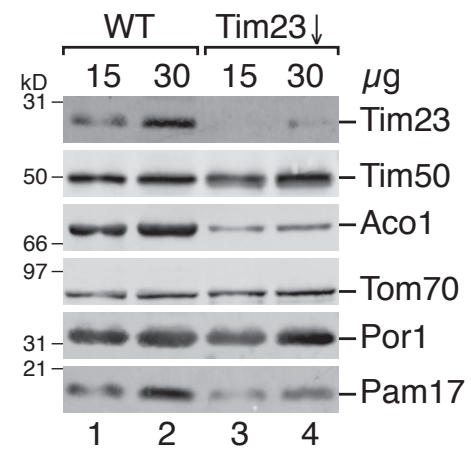

E

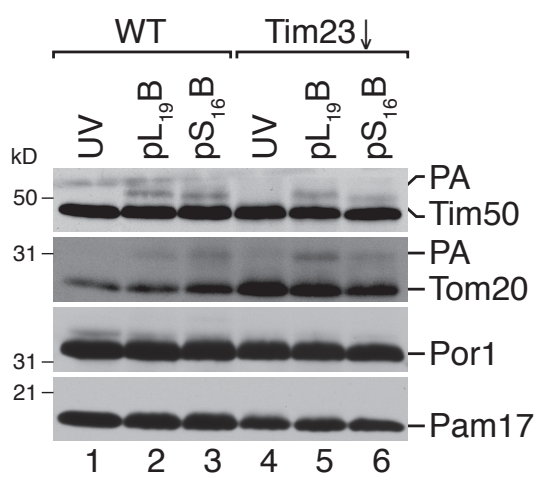

B

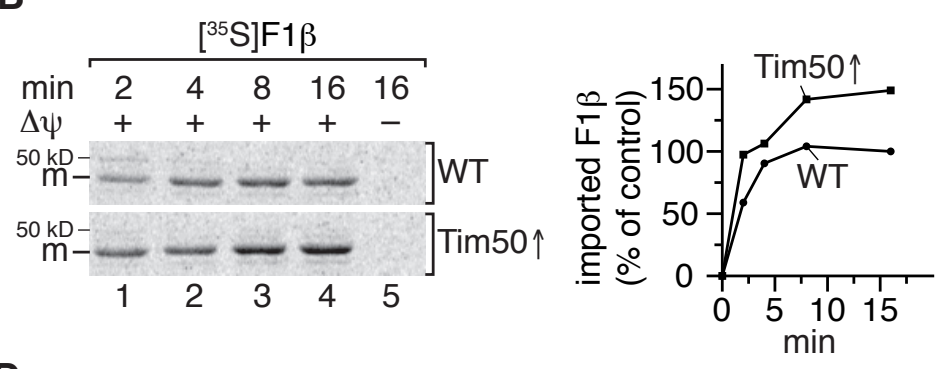

D

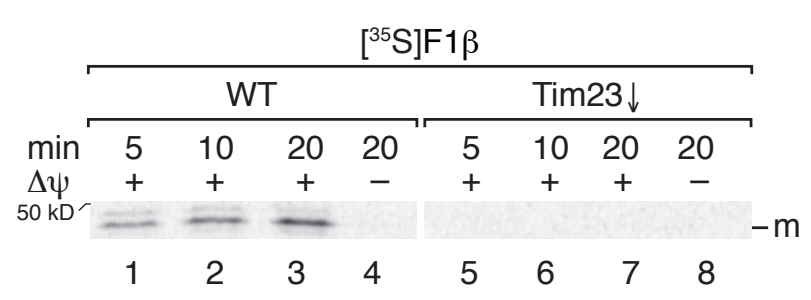

$\mathbf{F}$

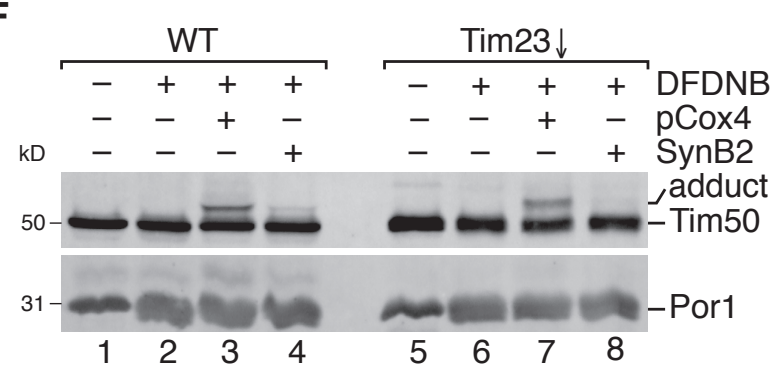

Fig. 22: Presequence binding to Tim50 is independent of Tim23 - A In organello photocrosslinking using $\mathrm{pL}_{19} \mathrm{~B}$ and $\mathrm{pS}_{16} \mathrm{~B}$ in isolated WT and Tim50个 mitochondria. Samples were analyzed by SDS-PAGE and western blotting. B $\left.{ }^{35} \mathrm{~S}\right]$ Met labeled F1 $\beta$ was imported into WT and Tim50个 mitochondria for the indicated time. Samples were PK treated and analyzed by SDS-PAGE and autoradiography. Quantification of the autoradiogram. The $16 \mathrm{~min}$ time point of the WT was set to $100 \%$. $\mathrm{m}$ mature (processed) form. C Steady state protein levels of isolated WT and Tim23 $\downarrow$ mitochondria. Samples were analyzed as in A. D F1 $\beta$ import as in B. Samples were analyzed by SDS-PAGE and autoradiography. m - mature (processed) form. E In organello photocrosslinking in isolated WT and Tim23 $\downarrow$ mitochondria. Samples were analyzed as in A. F Chemical crosslinking of $20 \mu \mathrm{M}$ peptides with $1 \mathrm{mM}$ DFDNB for 30 min. Samples were analyzed as in A. 
et al., 2009). Hence, the efficiency of precursor binding and transport to and through the TOM complex was assessed. To this end radiolabeled Su9-DHFR (consisting of a fusion between the presequence of subunit 9 of the $N$. crassa $\mathrm{F}_{1} \mathrm{~F}_{0}$-ATP synthase) was imported in the absence of $\Delta \psi$ hence preventing transport across the inner membrane. Additionally, methotrexate was supplemented to stabilize the DHFR fold, preventing it from passing the outer membrane. Under these conditions the precursor is stably arrested in the TOM complex (Kanamori et al., 1999). The precursor was arrested with the same efficiency in the Tim50 $\downarrow+\operatorname{Tim} 50^{\mathrm{HA}}$ and $\operatorname{Tim} 50 \downarrow+\operatorname{Tim} 50^{\triangle \mathrm{PBD}-\mathrm{HA}}$ mitochondria (Figure 23.C lanes 1 and 2). Both intermediates were of similar stability as equal amounts of a PK protected DHFR fragment were released into the supernatant upon PK treatment (lanes 3 and 4). Hence the absence of the presequence binding domain impairs import into mitochondria but does not affect the stability of TOM translocation intermediates indicating a role of the PBD in the translocation across the inner membrane.

To test this, an antibody against Tim50 $0^{\mathrm{PBD}}$ was affinity purified and used to inhibit import into mitoplasts (Figure 23.D). Import was significantly inhibited by the $\alpha$-Tim50 ${ }^{\mathrm{PBD}}$ antibody, while it was largely unaffected by control IgGs. As an alternative approach Tim50 $\downarrow+\operatorname{Tim} 50^{\mathrm{HA}}$ and $\operatorname{Tim} 50 \downarrow+\operatorname{Tim} 50^{\Delta \mathrm{PBD}-\mathrm{HA}}$ mitochondria were converted to mitoplasts by swelling in a hypotonic buffer and subsequently matrix targeted precursor proteins were imported (Figure 23.E and F). Similar to the results obtained for the import into these mitochondria, the import into mitoplasts was reduced to $25 \%$ in the mutant. Swelling efficiencies were similar for both strains (Figure 23.G). The $25 \%$ residual import can probably be attributed to the residual levels of the chromosomally encoded Tim50 (Figure 21.B lanes 3 and 4).

In order to exclude that the import defects seen are due to a reduced membrane potential, $\Delta \psi$ measurements using $\mathrm{DISC}_{3}(5)$ were performed. No significant difference between both types of mitochondria were observed (Figure 24), indicating that deletion of the presequence binding domain affects the import of matrix targeted presequence substrates across the inner membrane due the disturbed presequence recognition by Tim50, not a reduced $\Delta \psi$. Along this line, the import and assembly of the TIM22 substrate AAC $\left(\Delta \psi\right.$ dependent) was even slightly enhanced in the Tim50 $\downarrow+\operatorname{Tim} 50^{\Delta \mathrm{PBD}-\mathrm{HA}}$ mitochondria (Schulz et al., 2011), an unexplained effect seen when the TIM23 or PAM complex is compromised (Geissler et al., 2002; Yamamoto et al., 2002; Frazier et al., 2004). 
A

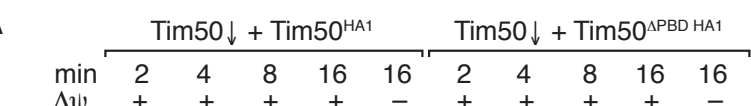

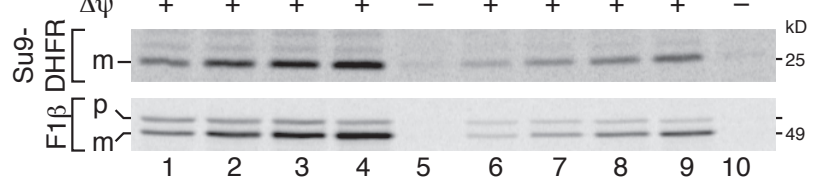

B

C Tim50 $\downarrow$ mitochondria, $-\Delta \psi$
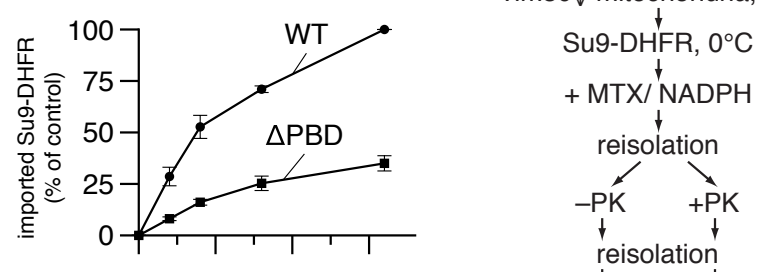

D
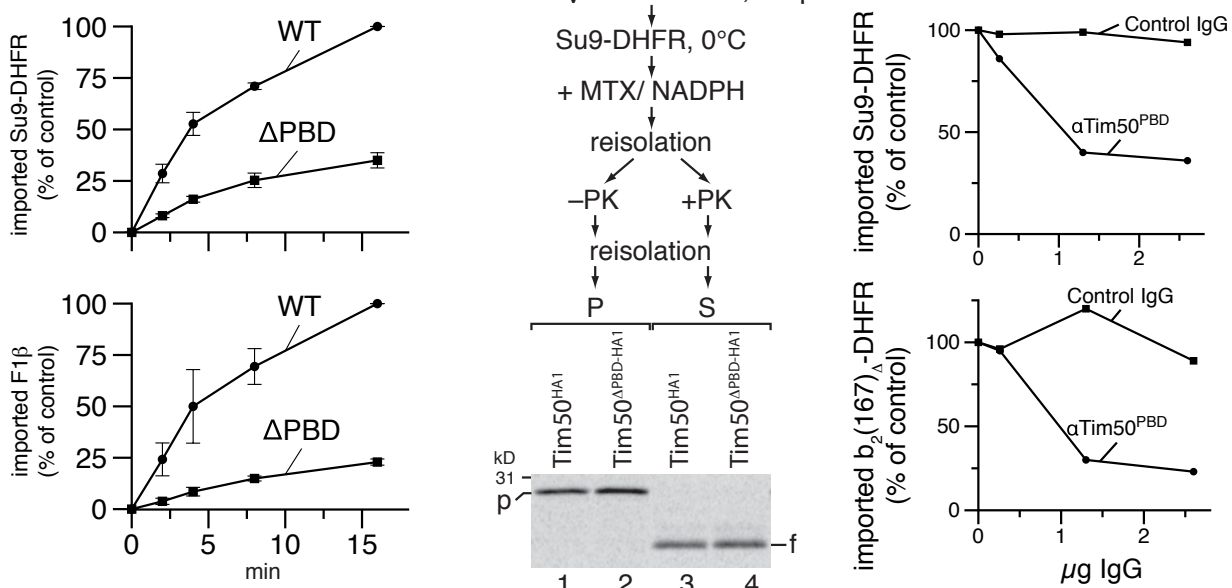

$E$
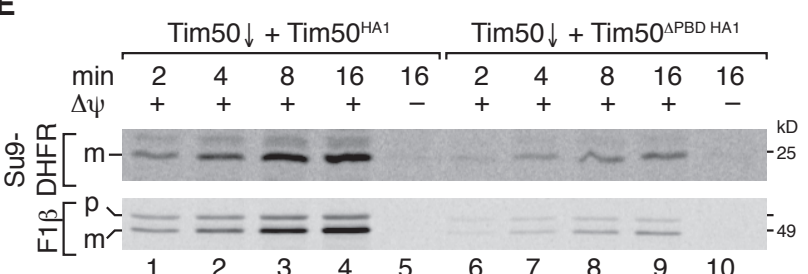

G
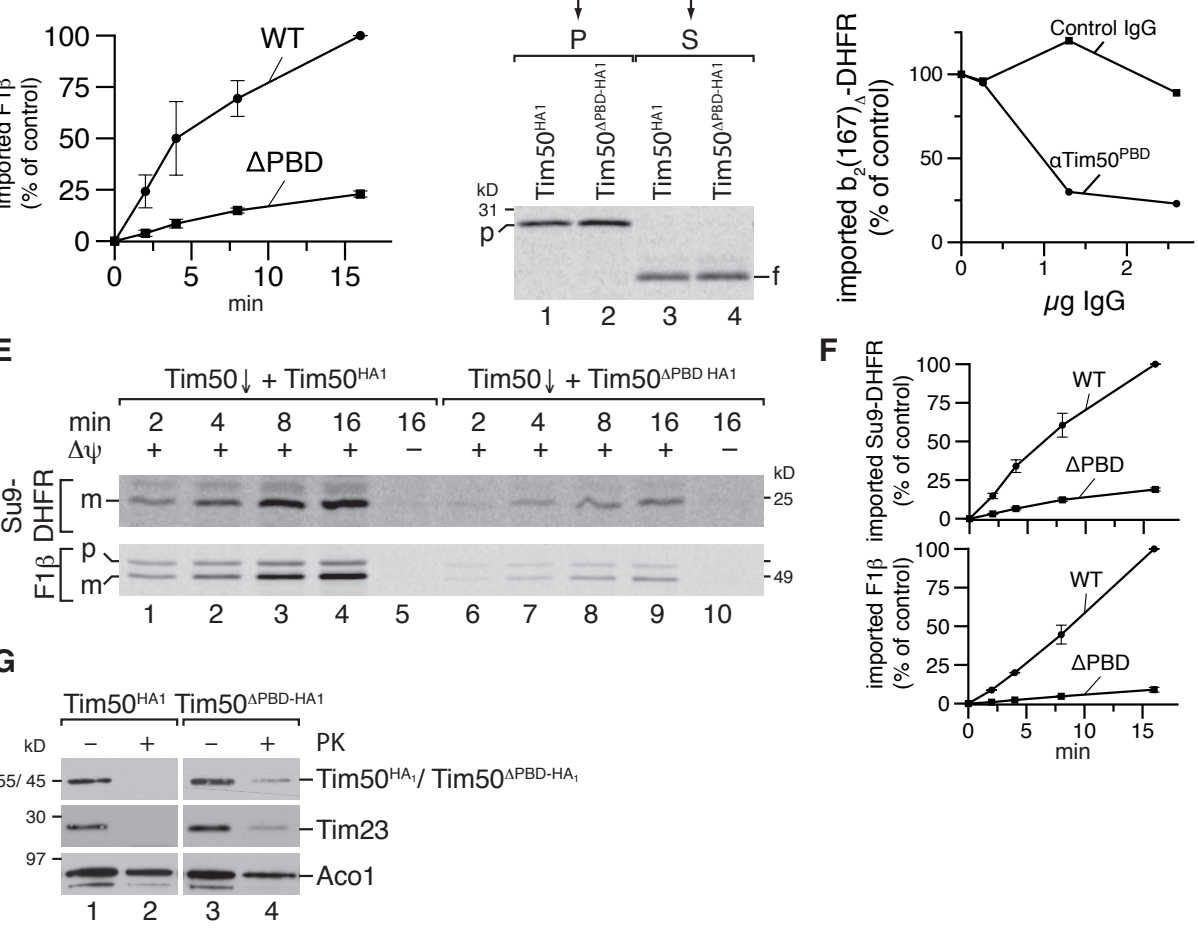

Fig. 23: Deletion of the Tim50 ${ }^{\mathrm{PBD}}$ affects presequence import. - A $\left.{ }^{35} \mathrm{~S}\right] \mathrm{Met}$ labeled F1 $\beta$ and Su9-DHFR were imported into isolated Tim50 $\downarrow+$ Tim50 ${ }^{\mathrm{HA}}$ and Tim50 $\downarrow+$ Tim $50^{\triangle \mathrm{PBD}-H A}$ mitochondria. Samples were PK treated and analyzed by SDS-PAGE and autoradiography. p - precursor, $\mathrm{m}$ - mature form. B Imports as described in A were quantified. Import into the WT at 16 min was set to $100 \%$ $(\mathrm{n}=3, \mathrm{SEM})$. C Radiolabeled Su9-DHFR was imported into isolated mitochondria in the absence of a membrane potential and subsequently diluted into a NADPH and methotrexate containing buffer. After reisolation, mitochondria were PK treated. Samples analyzed as in A. P - pellet, S - supernatant, p - precursor, f - PK stable DHFR fragment. (Continued on next page.) 
Fig. 23: (Continued from previous page.) - D Radiolabeled Su9-DHFR and $\mathrm{b}_{2}(167)_{\Delta^{-}}$ DHFR were imported into mitochondria or mitoplasts for $15 \mathrm{~min}$ in the presence of the indicated amounts of affinity purified $\alpha$-Tim $50^{\mathrm{PBD}}$ antibodies. Samples were PK treated and analyzed as in A. $100 \%$ import in the absence of antibodies. The import was calculated as the percentage of import into mitoplasts of the corresponding import into mitochondria. E Radiolabeled F1 $\beta$ and Su9-DHFR were imported into $\operatorname{Tim} 50 \downarrow+\operatorname{Tim} 50^{\mathrm{HA}}$ or Tim50 $\downarrow+\operatorname{Tim} 50^{\Delta \mathrm{PBD}-\mathrm{HA}}$ mitoplasts. Samples were PK treated and analyzed as in A. p - precursor, $\mathrm{m}$ - mature form. F Imports as described in E were quantified. Import into the WT at 16 min was set to $100 \%(\mathrm{n}=3$, SEM). G $\operatorname{Tim} 50 \downarrow+\operatorname{Tim} 50^{\mathrm{HA}}$ or Tim50 $\downarrow+\operatorname{Tim} 50^{\Delta \mathrm{PBD}-\mathrm{HA}}$ mitochondria were converted to mitoplasts and PK treated. Samples were analyzed by SDS-PAGE and western blotting.

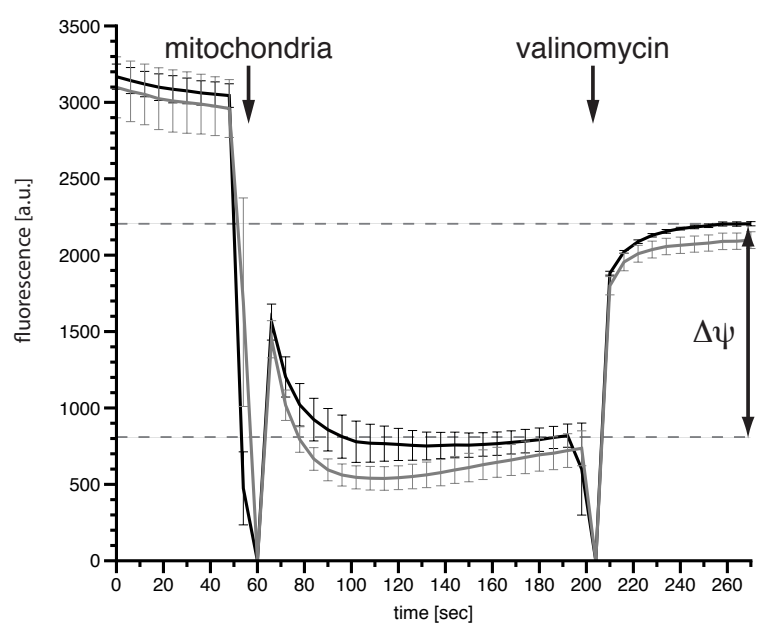

Fig. 24: Tim50 $\downarrow+\operatorname{Tim} 50^{\mathrm{HA}}$ and Tim50 $\downarrow+\operatorname{Tim}^{\mathrm{S}} 0^{\Delta \mathrm{PBD}-\mathrm{HA}}$ have a similar membrane potential - Isolated Tim50 $\downarrow+\operatorname{Tim} 50^{\mathrm{HA}}$ (black curve) and Tim50 $\downarrow+\operatorname{Tim} 50^{\Delta \mathrm{PBD}-\mathrm{HA}}$ mitochondria (gray curve) were subjected to membrane potential measurements using $\operatorname{DiSC}_{3}(5)$. Mitochondria were added after $50 \mathrm{~s}$, the membrane potential was dissipated using valinomycin after $200 \mathrm{~s}(\mathrm{n}=3, \mathrm{SEM})$. The membrane potential is reflected by the difference in fluorescence before and after valinomycin addition.

\subsubsection{The presequence is bound to Tim50 in a complex between Tim23 and Tim50}

Apart from the inter membrane space domain of Tim50, the second half of the IMS domain of Tim23 (residues 71-84) is also able to bind to presequences (Bauer et al., 1996; de la Cruz et al., 2010). This is also the region where both IMS domains interact with each other, a process responsible for closure of the Tim23 channel under resting conditions (Yamamoto et al., 2002; Geissler et al., 2002; Meinecke et al., 2006; Alder et al., 2008b; 
Tamura et al., 2009). To clarify whether Tim23 could bind to Tim50 and the presequence at the same time, Tim $50^{\mathrm{IMS}}$ was titrated into a reaction of Tim $23^{\mathrm{IMS}}$, presequence peptide and chemical crosslinking was performed. While the Tim23-Tim50 adduct increased with increasing Tim50 concentrations, the Tim23-presequence adduct decreased indicating that Tim23 binds the presequence and Tim50 mutually exclusive. Interestingly, this effect was also seen with Tim50 $\triangle \mathrm{PBD}$ (Schulz et al., 2011).

A handover of the presequence between both proteins would require a trimeric complex during the process. In order to identify this complex, equimolar amounts of Tim $23^{\text {IMS }}$ and Tim50 ${ }^{\mathrm{IMS}}$ were mixed with a biotin labeled ALDH presequence peptide and subjected to chemical crosslinking. A prominent Tim50-presequence as well as a weak Tim23presequence crosslinking adduct were detected (Figure 25). The weak crosslinking efficiency between Tim23 and the presequence compared to Tim50 and the presequence could indicate that the affinity of both receptors for the signal is significantly different. This was confirmed by other studies showing a ten fold difference in affinity (de la Cruz et al., 2010; Marom et al., 2011; Lytovchenko et al., 2013). Additionally, a complex containing Tim50, Tim23 as well as presequence peptide was identified.

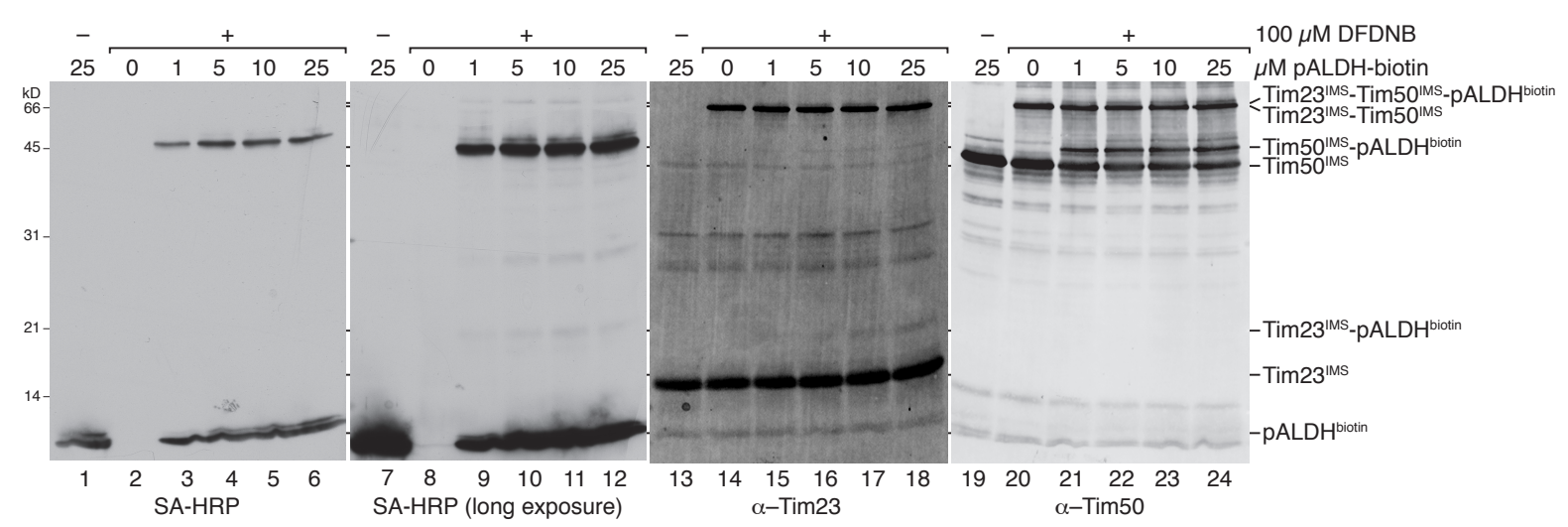

Fig. 25: A trimeric complex between Tim23, Tim50 and the presequence - $1 \mu \mathrm{M}$ of the purified IMS domains of Tim23 and Tim50 were mixed with the indicated concentrations of pALDH-biotin. After chemical crosslinking using $100 \mu \mathrm{M}$ DFDNB for $30 \mathrm{~min}$ on ice the samples were analyzed by SDS-PAGE and western blotting as indicated. 


\subsection{Dynamics of TIM23 subunits during transloca- tion of a substrate}

The TIM23 complex consists of several subunits, core components like Tim23, Tim17, Tim50 and Mgr2, as well as the additional subunit Tim21. The initial transport step across the inner membrane is driven by the membrane potential that acts on the presequence. Transport of inner membrane proteins containing a folded domain before the stop transfer signal as well as matrix targeted proteins requires the action of the ATP driven import motor (PAM, presequence translocase associated motor). This import motor is based on the mtHsp70 and its cochaperone Pam18 and the soluble nucleotide exchange factor Mge1. Additional subunits include the tethering factor Tim44, and the regulatory proteins Pam16 and Pam17. Hence, in total eleven different proteins are involved in the process of protein translocation into the mitochondrial matrix by the TIM23 complex.

Within the TIM23 several dynamic interactions are know. For example, the interactions between Tim21 and Tim50 as well as Tom22 and Tim50 show a decreased crosslinking efficiency in the presence of a presequence peptide (Shiota et al., 2011; Lytovchenko et al., 2013). Furthermore the association of the Hsp70 ATP with Tim44 is known to be regulated by Mge1 (Schneider et al., 1996; Liu et al., 2003).

It is unknown, how stable the association of these different subunits with the translocase is during the transport process. To answer this question an assay was designed that allowed to monitor the integration of subunits into the active translocase, indicative of exchange of translocase subunits during transport.

\subsubsection{Integration of subunits into the TIM23 and PAM complex}

The TIM23 complex can be isolated using a protein A (PA) tag fused to the N-terminus of Tim23 (Geissler et al., 2002). To analyze the integration of subunits into the resting translocase all subunits were radiolabeled in vitro and imported into mitochondria containing Tim $23^{\mathrm{PA}}$. Subsequently, the complex was isolated under mild conditions using digitonin. Except Hsp70 all tested subunits (Tim17, Tim21, Tim23, Tim44, Tim50, Pam16, Pam17, Pam18, Mgr2) integrated into the translocase (Figure 26.A). The isolation was specific, as substrates like F1 $\beta$ or Su9-DHFR as well as control proteins like Por1, Atp20 or Atp21 did not co-purify with the TIM23 complex. Quantification of the 
autoradiogram (normalized to Tim17) revealed high co-isolation efficiencies for Tim17 and Pam17 and a slightly lower efficiency for Tim21 indicating efficient recruitment of the imported proteins with the translocases in the inner membrane (Figure 26.B). Tim23, Tim50 and Mgr2 as well as the PAM subunits Tim44, Pam16 and Pam18 co-purified with a lower efficiency indicating a slower recruitment to TIM23 in comparison to Tim17. As a control, western blotting was performed and indicated the successful co-isolation of TIM23 and PAM subunits as well as the specificity of the isolation.

Hence, under these experimental conditions the integration of most TIM23 and PAM subunits into their respective complex could be analyzed.

\subsubsection{Generation of a TOM-TIM23 supercomplex by arrest of $\mathrm{b}_{2}(167)_{\Delta}$-DHFR}

\subsubsection{Arrest of chemical amounts of $b_{2}(167)_{\Delta}$-DHFR inhibit import}

In order to assay the incorporation of subunits into the active translocase, this active state needs to be stable enough to withstand biochemical purification procedures. The fusion protein $b_{2}(167)_{\Delta}$-DHFR consists of the N-terminal part of the cytochrome $b_{2}$ lacking the residues of the transmembrane segment $(\Delta 47-65)$ fused to the mouse dihydrofolate reductase. Due to the lack of the sorting signal, this precursor is transported in an import motor-dependent manner into the mitochondrial matrix. The DHFR can be stabilized by methotrexate (MTX) into a fold that can not be unfolded and hence imported by the inward driving force of the import motor, arresting the precursor during import in the TIM23 and TOM complexes (also referred to as supercomplex).

In order to generate quantitative amounts of active translocases, chemical amounts of the precursor were required. To this end the substrate was expressed in E. coli by IPTG induction and purified using cation exchange chromatography (Figure 27.A). The import-competence of the precursor was assayed by importing various amounts of it in the presence or absence of MTX and $\Delta \psi$ into isolated mitochondria (Figure 27.B). With both 0.2 and $0.6 \mathrm{nmol} / \mathrm{mg}$ of mitochondria, efficient import and generation of the MPP processed i form was observed when MTX was absent. In addition a smaller species was detected in a membrane potential dependent manner $\left(i^{*}\right)$ similar to previous reports (Stuart et al., 1994; Dekker et al., 1997; Ryan et al., 2001), which could be a product of degradation or another processing event, e.g. by Icp55 (Vögtle et al., 2009). The 
A

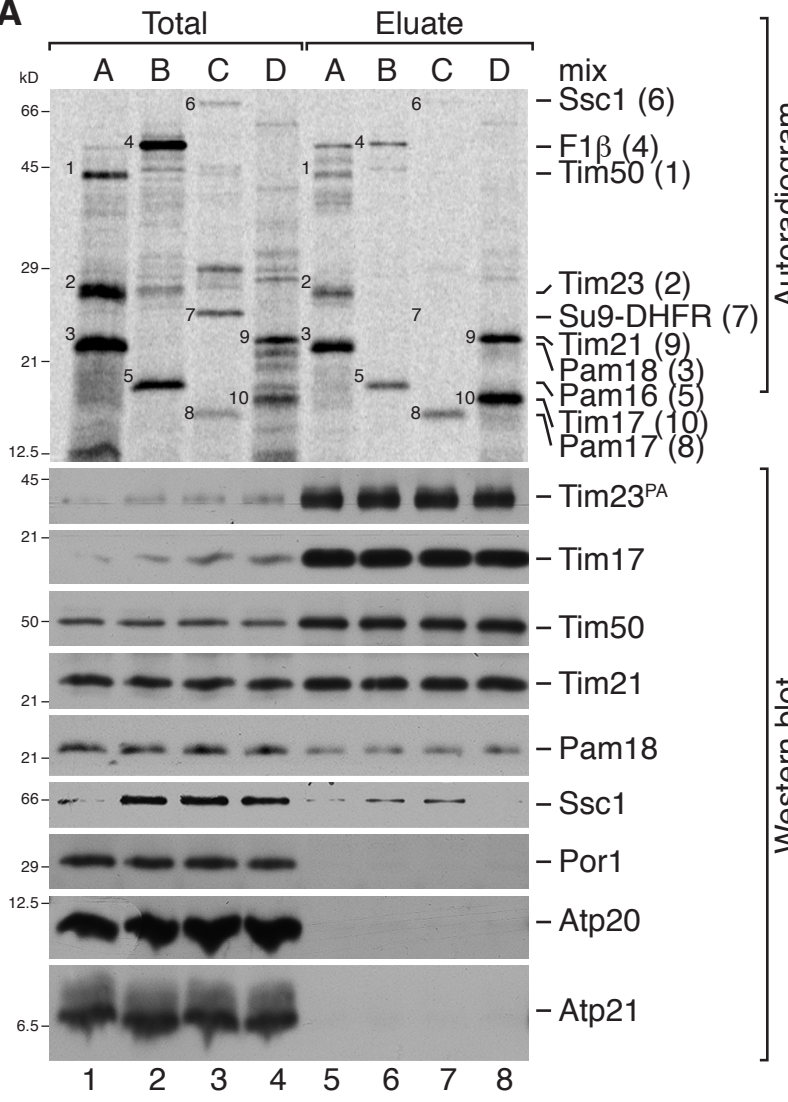

B

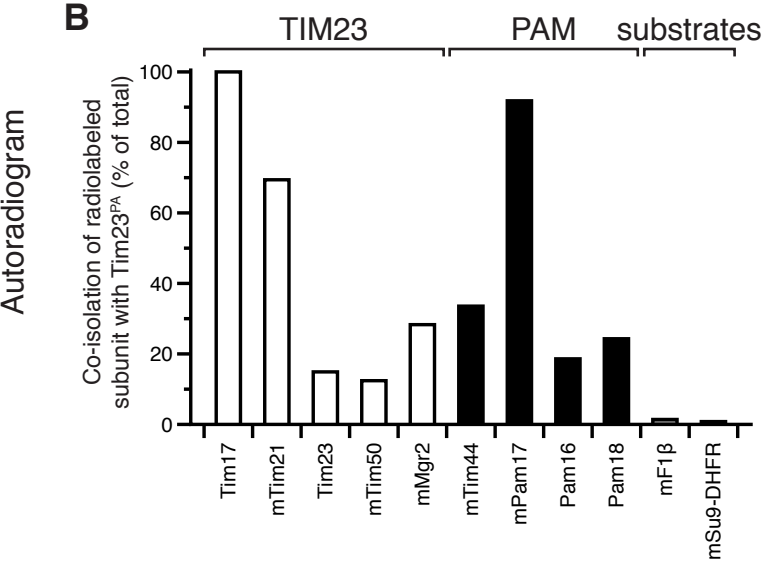

$\frac{0}{0}$
$\frac{5}{0}$
$\frac{10}{00}$
3

Fig. 26: Co-isolation of TIM23 and PAM subunits with Tim23 ${ }^{\text {PA }}$ - A TIM23 and PAM subunits as well as presequence-containing substrates were translated in vitro in the presence of $\left[{ }^{35} \mathrm{~S}\right]$ Met. The radiolabeled proteins were imported into Tim $23^{\mathrm{PA}}$ mitochondria for $45 \mathrm{~min}$. The reaction was stopped by the addition of AVO mix and PK treatment. Subsequently, the complex was isolated as described in A. Samples were analyzed by SDS-PAGE, autoradiography and western blotting. Total $5 \%$, eluate $100 \%$. B Quantification of the autoradiogram shown in B. 
processing by MPP generates a YG N-terminus that could be stabilized by cleavage of Icp55 generating a N-terminal Gly.

In order to estimate the amounts needed for a complete block of all TIM23 import sites different amounts of $\mathrm{b}_{2}(167)_{\Delta}$-DHFR were arrested in the presence of MTX for 15 min. After reisolation radiolabeled substrates were imported (Figure 27.C). Using $0.5 \mathrm{nmol}$ per mg of mitochondria resulted in $50 \%$ inhibition of the import of F1 13 and Pam18 $\left(50 \% \mathrm{~b}_{2}\right.$ saturation). Saturation was reached when using $1.5 \mathrm{nmol} / \mathrm{mg}$ mitochondria $\left(100 \% \mathrm{~b}_{2}\right)$. This was approximately twice as much as what Ryan et al. (2001) reported to be used for saturation and could be due to a lower import competence of the preparation.

In conclusion, $\mathrm{b}_{2}(167)_{\Delta}$-DHFR can be isolated from E. coli in an import competent state that can be used to generate a TOM-TIM23 supercomplex and saturate the TIM23 import sites.

\subsubsection{Isolation of the TOM-TIM23 supercomplex}

Upon successful formation of the supercomplex the precursor's C-terminal DHFR domain is tightly pulled against the TOM complex, it spans through the TOM and TIM23 complex and is held in the N-terminal region by TIM $23^{\text {MOTOR }}$.

In order to visualize the supercomplex, $b_{2}(167)_{\Delta}$-DHFR was imported in the absence or presence of MTX and the samples were solubilized using digitonin and subjected to BN-PAGE. The TIM23 complexes detected in the absence of precursor and MTX were quantitatively shifted to higher molecular weight upon import in the presence of MTX (Figure 28.A). The shifted complexes co-migrated with an upshifted fraction of the TOM complex (right side) indicating a successfully generated supercomplex. Hence, as previously determined by Dekker et al. (1997), there are more copies $(\sim 4 \mathrm{x})$ of the TOM complex compared to the TIM23 complex.

In addition to the separation by BN-PAGE the complex can be isolated in order to analyze the subunit composition. To this end, $\mathrm{b}_{2}(167)_{\Delta}$-DHFR was arrested in mitochondria containing a His10 tagged Tom22 in the absence or presence of MTX and the supercomplex was isolated by IMAC (Figure 28.B). Under both conditions the central subunit of the TOM complex, Tom40, co-isolated with Tom $22^{\text {His10 }}$. However, TIM23 and PAM subunits were only co-isolated upon arrest with MTX. The amount of TIM23 subunits co-isolated with Tom $22^{\mathrm{His} 10}$ correlated with the amount of $\mathrm{b}_{2}(167)_{\Delta}$-DHFR arrested (Figure 28.C). 

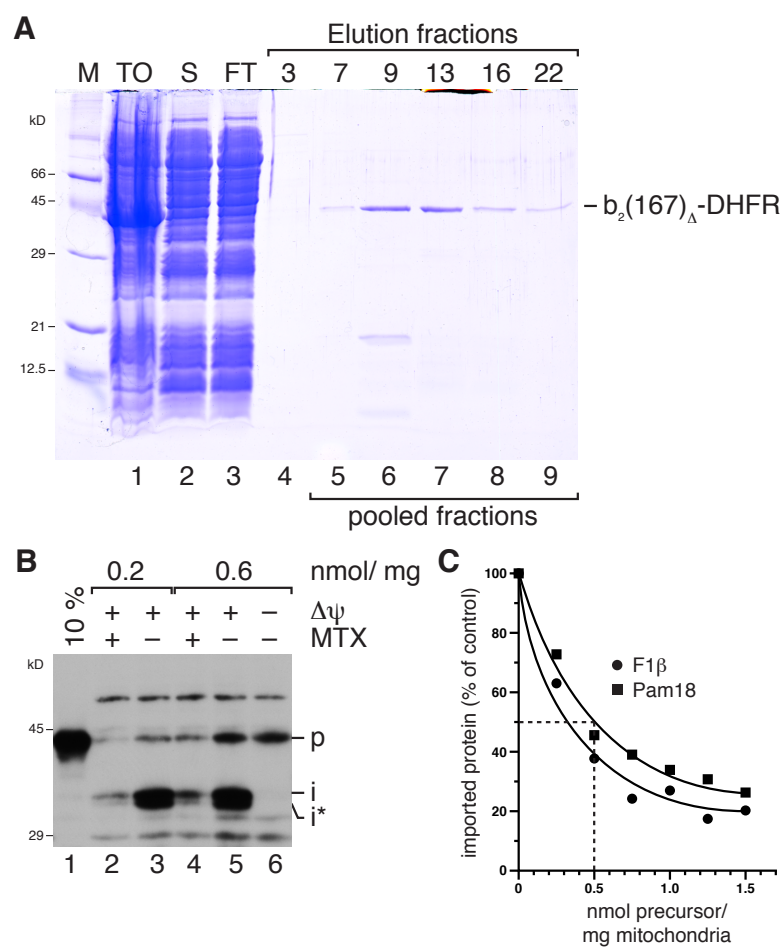

Fig. 27: Chemical amounts of $b_{2}(167)_{\Delta}$-DHFR block presequence import. - A $\mathrm{b}_{2}(167)_{\Delta}$-DHFR was expressed in E. coli and purified using cation exchange chromatography. TO - total, S - supernatant, FT - flow through. B The indicated amounts of purified $\mathrm{b}_{2}(167)_{\Delta}$-DHFR were imported into isolated mitochondria in the presence or absence of MTX and a membrane potential for $20 \mathrm{~min}$. Import was stopped by by addition of AVO and PK treatment. Samples were analyzed by SDS-PAGE and western blotting using $\alpha$-DHFR serum. $\mathrm{p}$ - precursor, i - intermediate, $i^{*}$ - degradation product of the intermediate. C Indicated amounts of $b_{2}(167)_{\Delta}$-DHFR were arrested in mitochondria for $15 \mathrm{~min}$. After reisolation radiolabeled F1 $\beta$ or Pam18 were imported for $30 \mathrm{~min}$. Subsequently, the membrane potential was dissipated by AVO and samples were PK treated and analyzed by SDS-PAGE and autoradiography. Quantification of the imported and processed (in the case of F1 $\beta$ ) protein is shown $(100 \%$ - imported in the absence of an arrested $\mathrm{b}_{2}(167)_{\Delta}$-DHFR). 
Hence, the TOM-TIM23 supercomplex can be stably generated and isolated, also at conditions when only $50 \%$ of the inner membrane import sites (TIM23) are occupied.
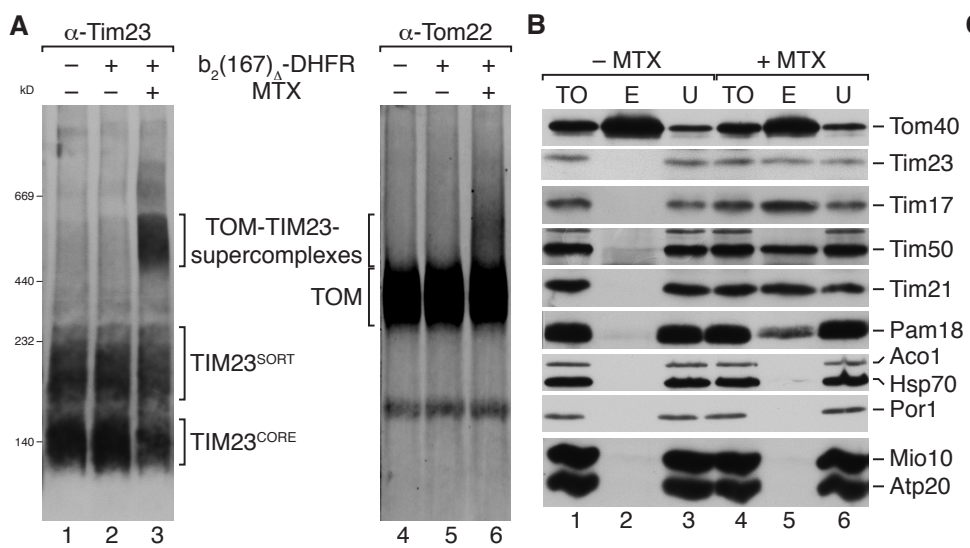

C

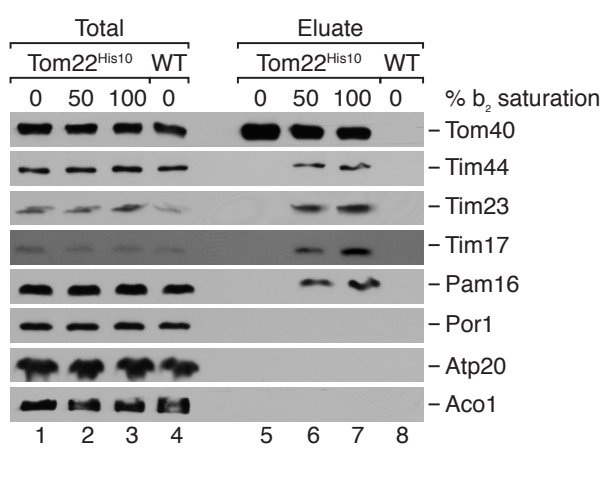

Fig. 28: Isolation and separation of the TOM-TIM23 supercomplex. - A $b_{2}(167)_{\Delta^{-}}$ DHFR was imported in the absence or presence of MTX into WT mitochondria. Subsequently, the samples were solubilized with digitonin and analyzed by BN-PAGE and western blotting. B Saturating amounts of $\mathrm{b}_{2}(167)_{\Delta}$-DHFR were imported into Tom $22^{\text {His10 }}$ mitochondria in the absence or presence of MTX. Subsequently, mitochondria were solubilized using digitonin and the TOM-TIM23 supercomplex was purified using Ni-NTA agarose. The sample was eluted with imidazole and analyzed by SDSPAGE and western blotting. To - total $5 \%$, E - eluate $100 \%$. C No, $0.5 \mathrm{nmol} / \mathrm{mg}(50 \%$ saturation) or $1.5 \mathrm{nmol} / \mathrm{mg}(100 \%) \mathrm{b}_{2}(167)_{\Delta}$-DHFR were imported into Tom $22^{\text {His10 }}$ mitochondria for $15 \mathrm{~min}$ at $25^{\circ} \mathrm{C}$ in the presence of MTX. Subsequently, the supercomplex was isolated and analyzed as in B. Total $5 \%$, eluate $100 \%$.

\subsubsection{Hsp70's ATPase activity is needed to stabilize the TOM-TIM23 su- percomplex}

It was previously shown that Hsp70 and Tim44 are not components of the BN-PAGE resolved TOM-TIM23 supercomplex (Dekker et al., 1997). This could however, solely be due to the dissociation of Hsp70 and Tim44 from the complex during the blue native electrophoresis, as Hsp70 is also easily lost during TIM23 complex isolations (Figure 26.A) (van der Laan et al., 2005). To address this, temperature sensitive alleles of Hsp70 (ssc1-2 and ssc1-3) were used (Figure 29.A) (Kang et al., 1990; Gambill et al., 1993). After induction of the phenotype by incubation for $15 \mathrm{~min}$ at $37^{\circ} \mathrm{C}$ no supercomplex was generated in both strains, in contrast to WT mitochondria. However, in these mutant 
mitochondria the import of a matrix targeted precursor was drastically reduced (Figure 29.B and C), explaining why no supercomplex was generated (Gambill et al., 1993).

A

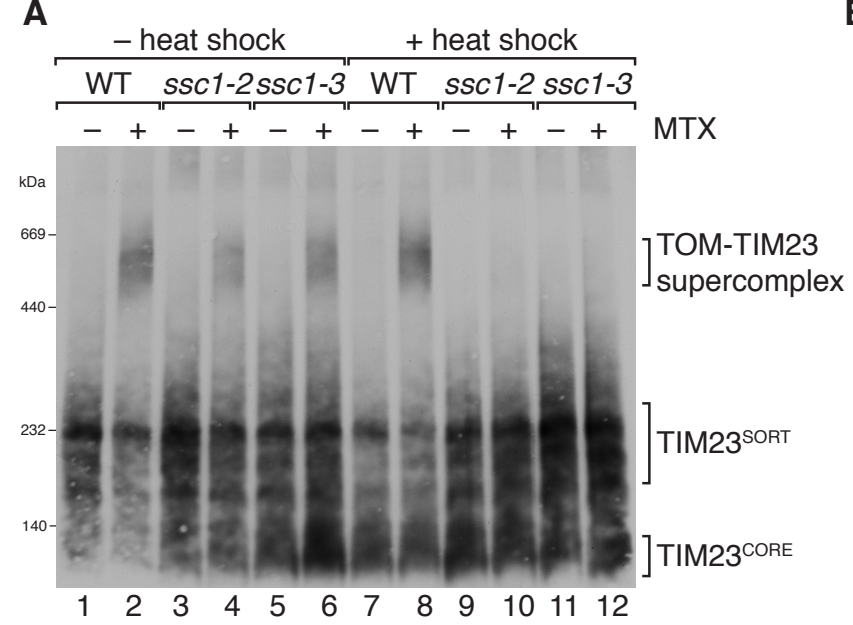

B

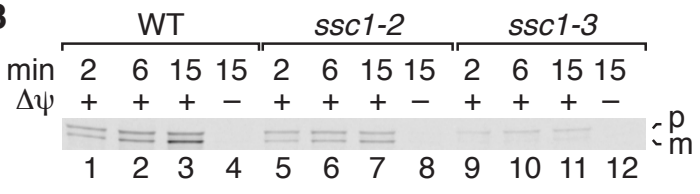

C

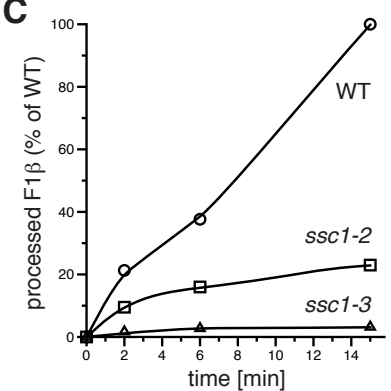

Fig. 29: Generation of the TOM-TIM23 supercomplex depends on the import efficiency - A WT, ssc1-2 and ssc1-3 mitochondria were incubated for $15 \mathrm{~min}$ at $37^{\circ} \mathrm{C}$ (+ heatshock) or on ice (- heatshock). Subsequently, $\mathrm{b}_{2}(167)_{\Delta}$-DHFR was imported for $15 \mathrm{~min}$ at $25^{\circ} \mathrm{C}$ in the presence or absence of MTX, mitochondria were solubilized with digitonin and analyzed by BN-PAGE and western blotting using $\alpha$-Tim23. B Import of radiolabeled F1 $\beta$ into WT, ssc1-2 and ssc1-3 after heatshock in the presence or absence of a membrane potential for the indicated times. The reactions were stopped by addition of AVO mix and PK treatment. Samples were anaylzed by SDS-PAGE. $\mathrm{p}$ - precursor, $\mathrm{m}$ - mature. C Quantification of the experiment in B. The import for 15 min in the BWT was set to $100 \%$.

To address the ability to generate and maintain the supercomplex in different mutants, $\mathrm{b}_{2}(167)_{\Delta^{-D H F R}}$ was arrested with MTX in WT, ssc1-3, tim44-804 and pam18-66 under normal conditions and after heatshock (Truscott et al., 2003; Frazier et al., 2004; Hutu et al., 2008). Additionally, the stability was monitored after a chase without membrane potential at restrictive temperature (Figure 30.A). As shown before, ssc1-3 did not generate a supercomplex after heatshock (Figure 30.B). Additionally, the amount of supercomplex generated under permissive conditions was reduced to $60 \%$ during a restrictive chase (Figure 30.C). Interestingly, tim44-801 showed a similar pattern. After heatshock only $30 \%$ of supercomplex were generated, and after a restrictive chase about $80 \%$ of the supercomplex were maintained (Figure 30.B and C). The pam18-66 mutant mitochondria generated about $70 \%$ and maintained only $50 \%$ of the supercomplex (Figure 30.B and C). 


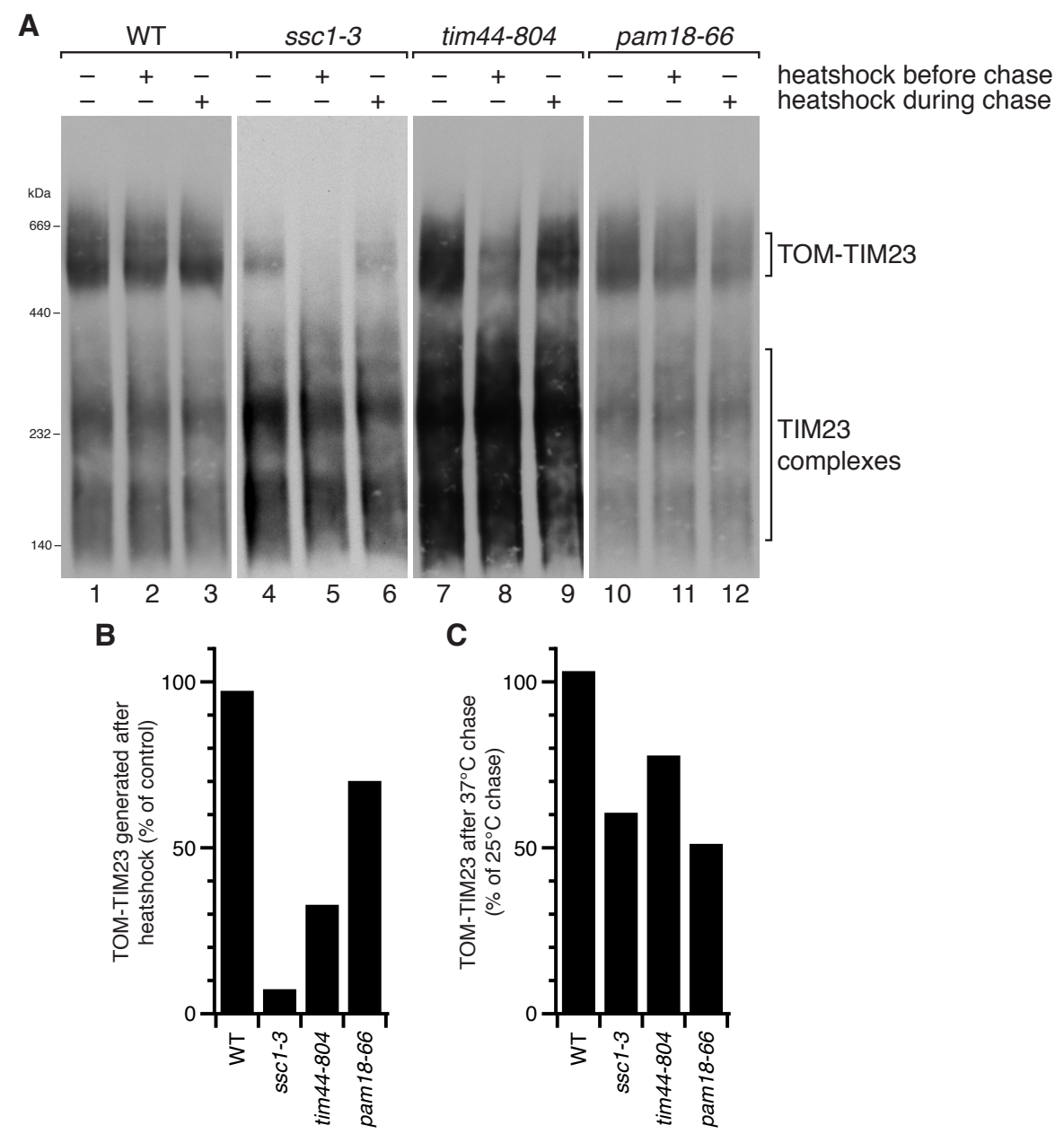

Fig. 30: Stability of the TOM-TIM23 supercomplex depends on the Hsp70 activity - A WT, ssc1-3, tim44-804 and pam18-66 mitochondria were incubated for $15 \mathrm{~min}$ at $37^{\circ} \mathrm{C}$ (+ heatshock) or on ice (- heatshock). Subsequently, $\mathrm{b}_{2}(167)_{\Delta}$-DHFR was imported in the presence or absence of MTX for $15 \mathrm{~min}$ at $25^{\circ} \mathrm{C}$ and the membrane potential was dissipated using $100 \mu \mathrm{M}$ valinomycin. The reactions were chased at $25^{\circ} \mathrm{C}$ (- heatshock) or $37^{\circ} \mathrm{C}$ (+ heatshock) for $30 \mathrm{~min}$. Afterwards, the mitochondria were solubilized with digitonin and analyzed by BN-PAGE and western blotting. B Quantification of the TOM-TIM23 supercomplex from A. The amount of supercomplex generated after heatshock is shown $(100 \%$ - supercomplex generated without heatshock). C Quantification of the TOM-TIM23 supercomplex from A. The amount of supercomplex retained after the heatshock chase is shown $(100 \%$ - supercomplex retained during a $25^{\circ} \mathrm{C}$ chase). 
In order to analyze the cause of this reduction in supercomplex, the TIM23 complex composition in these mutants was analyzed by $\alpha$-Tim23 co-immunoprecipitations. All TIM23 and PAM subunits were specifically precipitated under these conditions (Figure 31.A). Control serum did not precipitate these proteins and control proteins of the outer membrane (Por1), inner membrane (Atp20) and matrix (Aco1) were not co-isolated with Tim23. Except Hsp70 none of the other subunits showed a significant change in complex association in dependence of the ATP status (Figure 31.B). Hsp70 was lost from the translocase when ATP was present as shown before (Schneider et al., 1994; von Ahsen et al., 1995; Horst et al., 1996). Therefore, all following immunoprecipitations were performed without ATP and after incubation at restrictive temperature for the temperature sensitive mutants.

A

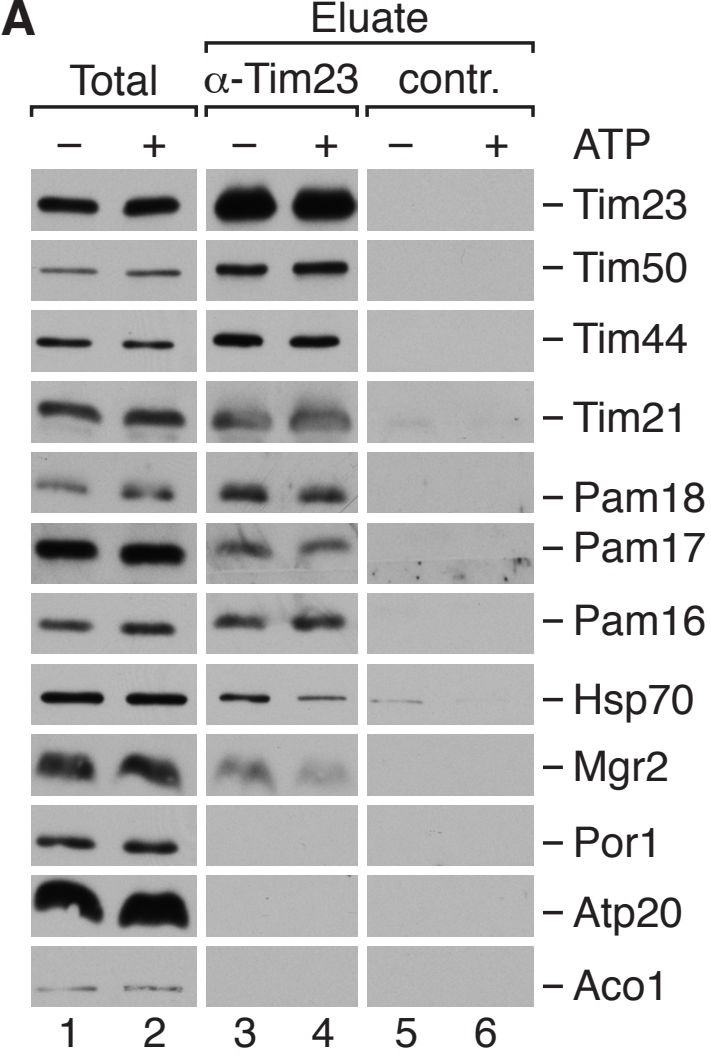

B

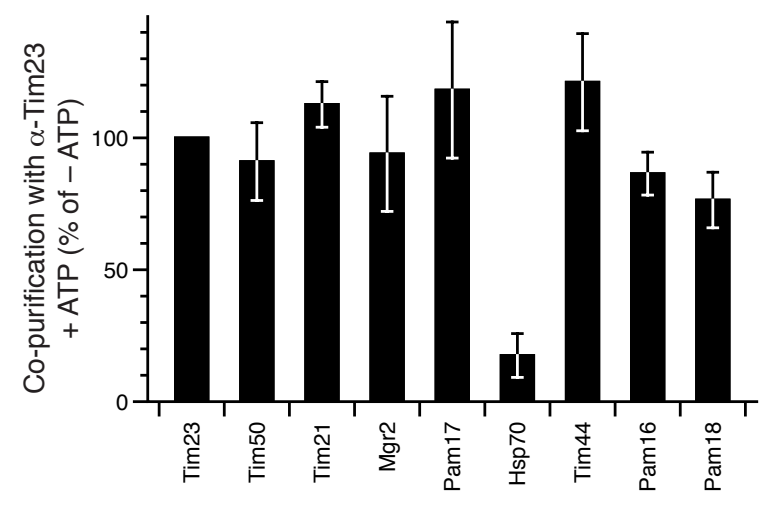

Fig. 31: TIM23 can be specifically isolated by $\alpha$-Tim23 co-immunoprecipitations A $\alpha$-Tim23 co-immunoprecipitation from digitonin solubilized mitochondria with or without ATP. An unrelated serum served as control. Samples were analyzed by SDSPAGE and western blotting. Total 5\%, eluate $100 \%$. B Quantification of A ( $\mathrm{n}=3$, SEM). 
Precipitation of Tim23 from the ssc1-3 mitochondria was slightly more efficient than in the corresponding WT (Figure 32.A). Taking this into account, similar amounts of the different subunits were isolated with the complex. Interestingly, the amount of Mgr2 observed in the ssc1-3 at steady state was significantly increased (Figure 32.A, lane 2). In the pam18-66 mutant mitochondria the immunoprecipitation revealed that both Pam16 and Pam18 were more abundant at steady state (Figure 32.B, lanes 1 and 2). However, only wild-type levels of Pam16 and reduced levels of Pam18 were precipitated with the complex (lanes 3 and 4). Interestingly, more Hsp70 but similar amounts of Pam17 were associated with the translocase.

The pam16-3 showed decreased Pam16 and Pam18 levels along with increased Tim50, Tim21, Pam17 and Mgr2 levels (Figure 32.C, lanes 1 and 2). These ratios were maintained in the precipitation (lanes 3 and 4). The decreased association of Pam18 with the translocase was a secondary effect of reduced Pam16 levels at the translocase, as in the pam18-66 mitochondria the Pam18 reduction had no effect on the Pam16 association.

Hence, in the pam18-66 and pam16-3 mutants the phenotype can be explained by the reduced abundance of regulatory subunits at the translocase, while in ssc1-3 the defect is due to a defective nucleotide binding domain in Hsp70 (Voos et al., 1996).

Interestingly, the matrix import into pam18-66 and pam16-3 was not significantly reduced, in contrast to defects reported (Figure 35.A) (Truscott et al., 2003; Frazier et al., 2004). However, the ssc1-3 displayed the drastic import defect expected (Figure 35.A) (Gambill et al., 1993).

In conclusion the Hsp70 ATPase activity, at least relying on Hsp70, Tim44 and Pam18, appears to be required for the stable maintenance of the TOM-TIM23 supercomplex.

\subsubsection{Integration of subunits into the active TOM-TIM23 su- percomplex}

\subsubsection{Tim44, Tim21, Pam18 and Tom20 integrate into the active TOM- TIM23 supercomplex}

In order to estimate the capability of different subunits to integrate into the active TIM23 translocase the following assay was designed. $\mathrm{b}_{2}(167)_{\Delta}$-DHFR was arrested with MTX at $50 \%$ saturation in order to maintain free TIM23 translocases. Subsequently, radiolabeled subunits were imported into these mitochondria and chased for $5 \mathrm{~min}$ after dissipation 

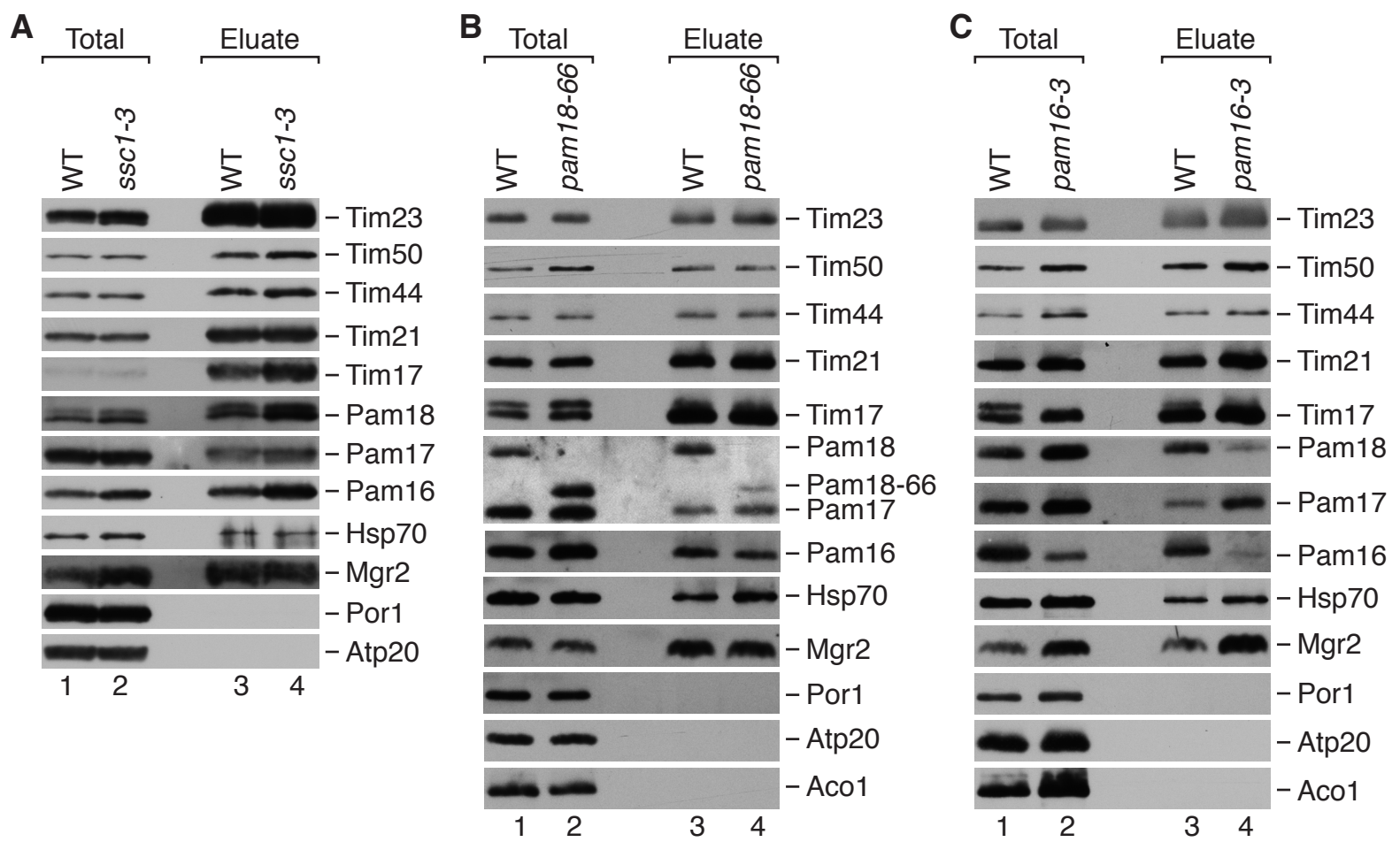

Fig. 32: Temperature-sensitive mutants of PAM components reveal reduced association with the TIM23 complex - After incubation for $15 \mathrm{~min}$ at $37^{\circ} \mathrm{C}$ digitonin solubilized mitochondria were subjected to $\alpha$-Tim23 co-immunoprecipitations. Samples were analyzed by SDS-PAGE and western blotting. Total $5 \%$, eluate $100 \%$. A WT and ssc1-3. B WT and pam18-66. C WT and pam16-3.

of the membrane potential. The supercomplex was isolated using Tom $22^{\text {His10 }}$ in the case of TIM23 and PAM subunits or Tim23 $3^{\mathrm{PA}}$ for TOM subunits. The detection of the radiolabeled protein in the eluate would only be expected if the unlabeled protein is also co-isolated (Figure 33.A).

Tim17 and Tim21 co-isolated with Tim23 ${ }^{\mathrm{PA}}$ independent of the arrested $\mathrm{b}_{2}(167)_{\Delta^{-}}$ DHFR, while Tom 20 and Tom40 were only isolated upon generation of the supercomplex (Figure 33.B, C). Radiolabeled, imported Tom40 was not able to integrate into the TOM complex while Tom20 did (Figure 33.G). The isolation efficiencies of the unlabeled and radiolabeled Tom20 were similar (Figure 33.G and $\mathrm{H}$ ).

Just like the pore forming subunit of the TOM complex Tom40, radiolabeled Tim23 was not co-isolated with Tom $22^{\text {His10 }}$ when the supercomplex was generated (Figure 33.D). The same was true for a TIM23 substrate, F1 $\beta$ (Figure 33.D and I). However, radiolabeled 
and imported Tim44, Pam18 and Tim21 specifically co-isolated with Tom22 $2^{\text {His10 }}$ under these conditions (Figure 33.E and F). Additionally, a slight co-isolation of Pam16 was observed (Figure 33.F). The respective unlabeled forms of these proteins were efficiently co-isolated as detected by western blot (Figure 33.D-F, I and J). As expected, TOM subunits like Tom40 co-isolated with Tom $22^{\text {His10 }}$ independent of the $b_{2}(167)_{\Delta}$-DHFR arrest (Figure 33.D-F and J).

Hence, regulatory subunits of the TIM23 and PAM complex, namely Tim44, Pam18 and Tim21 integrate into the TOM-TIM23 supercomplex, as does the peripheral TOM subunit Tom 20 . 

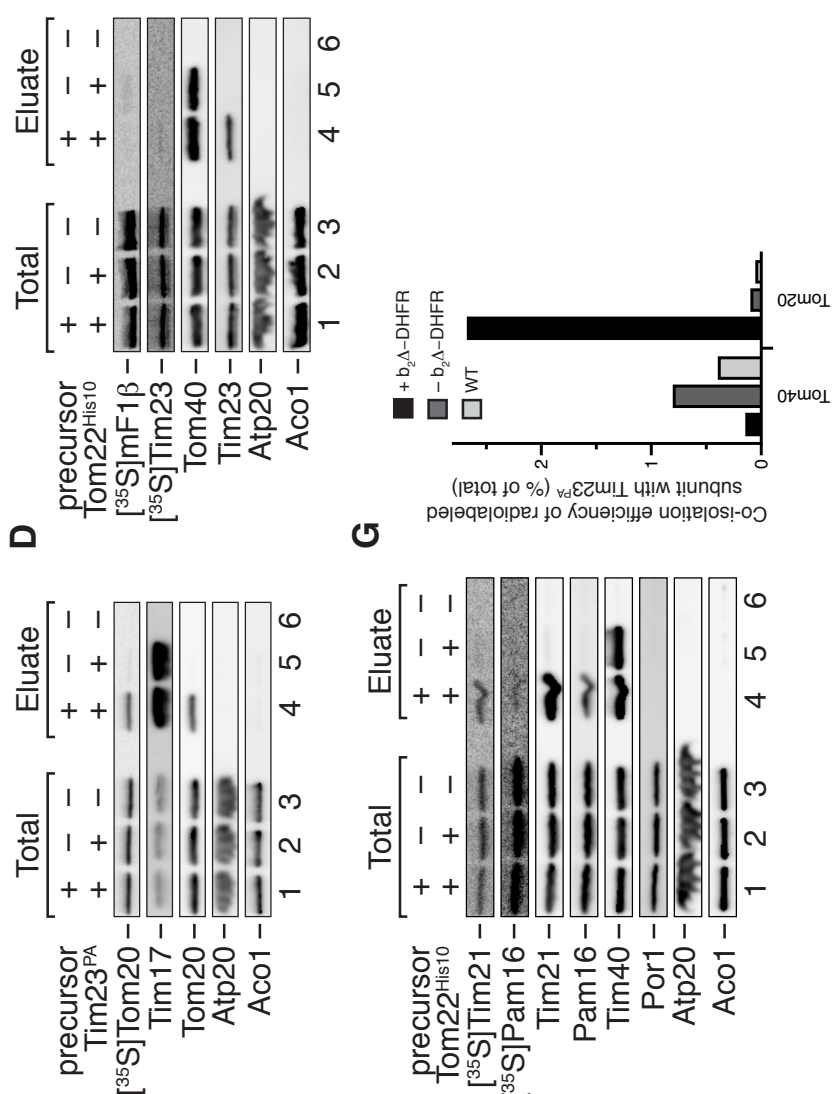

0

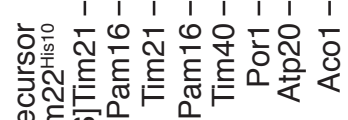

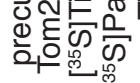

ᄂ
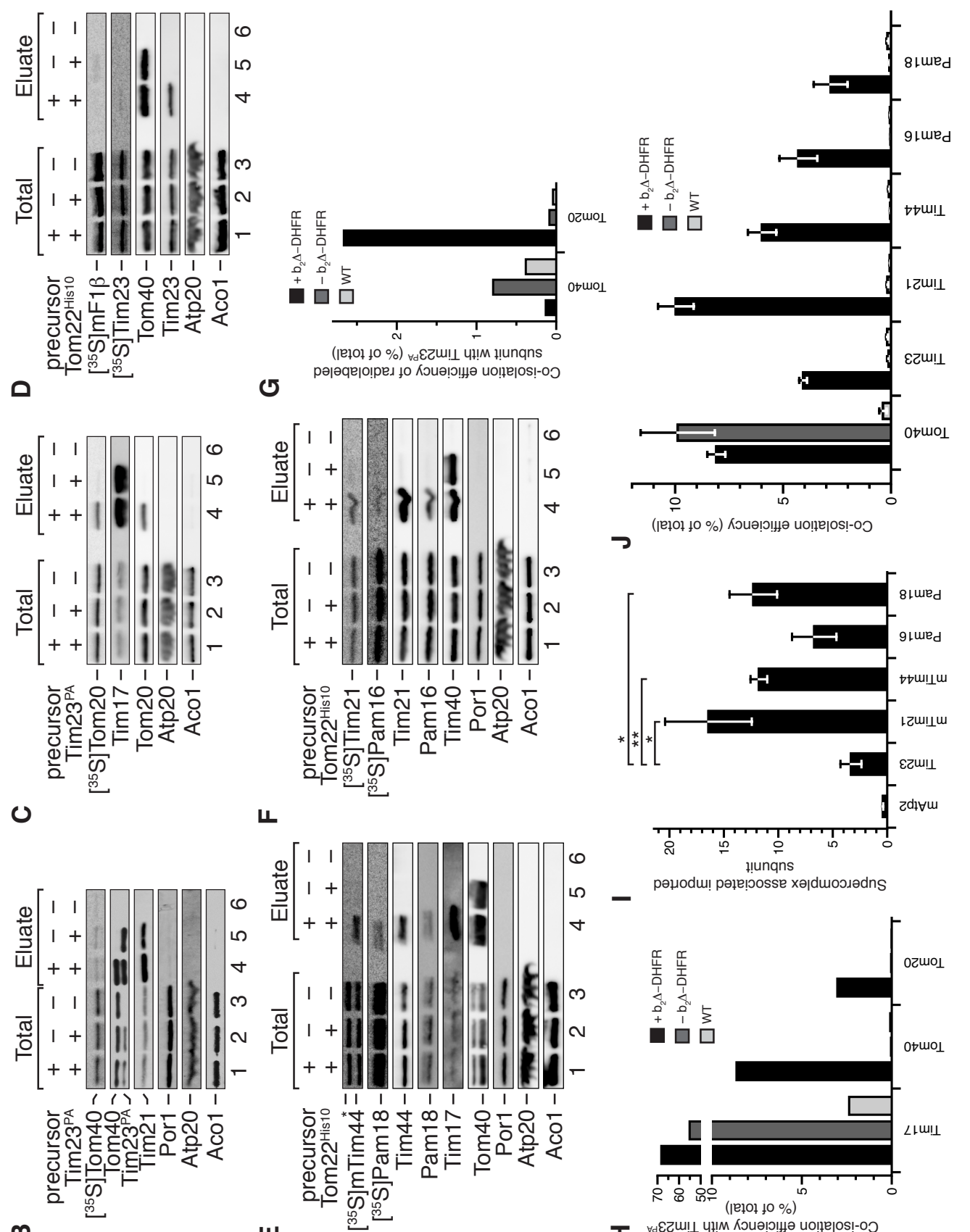

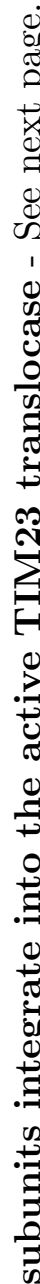

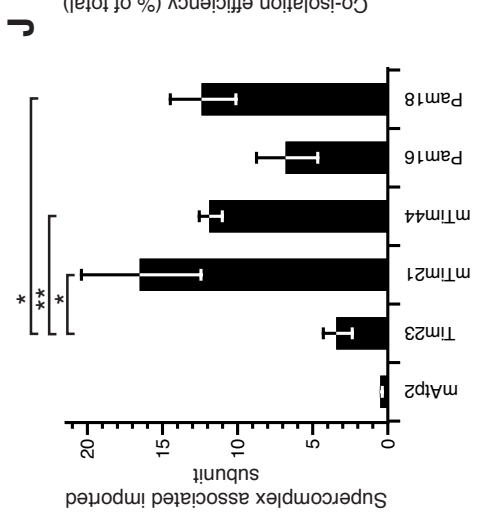

焉

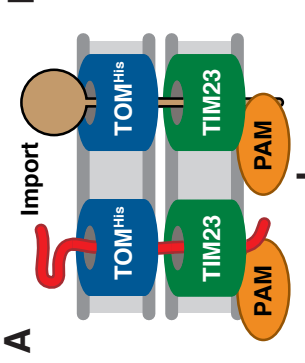

Ш

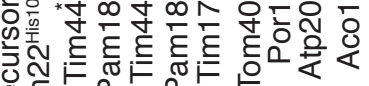

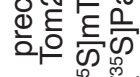

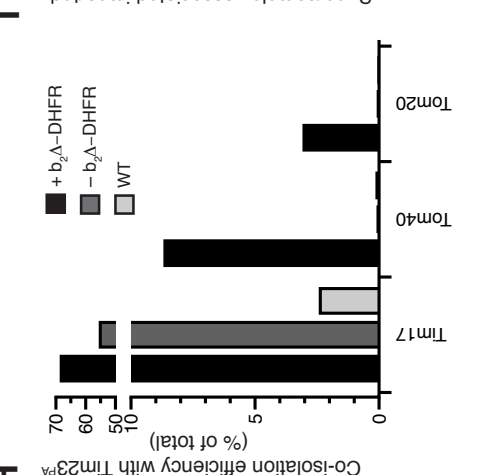

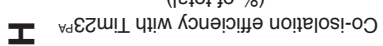

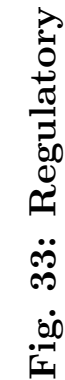


Fig. 33: (Continued from previous page.) - A Cartoon of the experimental setup. $50 \%$ saturating amounts of $\mathrm{b}_{2}(167)_{\Delta}$-DHFR were arrested in mitochondria. The remaining free translocases are used to import radiolabeled subunits of the TIM23 or PAM complex and their integration into the TOM-TIM23 supercomplex is tested by isolation of Tom $22^{\text {His } 10}$. B Integration of radiolabeled Tom 40 into the supercomplex, isolated by Tim $23^{\mathrm{PA}}$. Samples were analyzed by SDS-PAGE, autoradiography and western blot. Total $5 \%$, eluate $100 \%$. C Integration of radiolabeled Tom22 into the supercomplex, isolation and analysis as in B. Total $5 \%$, eluate $100 \%$. D Integration of radiolabeled Tim 23 and F1 $\beta$ into the supercomplex, isolated by Tom $22^{\text {His } 10}$. Samples were analyzed as in B. Total $5 \%$, eluate $100 \%$. E Integration of radiolabeled Tim44 and Pam18 into the supercomplex, isolation and analysis as in D. Total $5 \%$, eluate $100 \%$. F Integration of radiolabeled Tim21 and Pam16 into the supercomplex, isolation and analysis as in D. Total 5\%, eluate $100 \%$. G Quantification of experiments described in $\mathrm{B}$ and $\mathrm{C}$. The co-isolation of the radiolabeled subunits as percentage of the total are displayed for samples containing an arrested $\mathrm{b}_{2}(167)_{\Delta}$-DHFR $\left(+\mathrm{b}_{2}\right.$, black) or no precursor $\left(-b_{2}\right.$, dark gray) in Tim $23^{\mathrm{PA}}$ as well as no precursor in WT mitochondria (WT, light gray). H Quantification of experiments described in B and C. The coisolation of the non-labeled subunits as the percentage of the total are displayed as in G. I Quantification of experiments described in D, E and F. The supercomplex associated imported radiolabeled TIM23 and PAM subunits is shown (ratio of the co-isolation efficiency, as percent of the imported protein, of the Tom $22^{\mathrm{His} 10}$ isolation containing arrested $\mathrm{b}_{2}(167)_{\Delta}$-DHFR or lacking it, $\mathrm{n}=3$, SEM). Significance tested with one-tailed T-test for unequal variances: **, very significant $(\alpha=0.01) ;{ }^{*}$, significant $(\alpha=0.05)$. J Quantification of experiments described in D, E and F. The co-isolation of the non-labeled subunits with Tom $22^{\text {His10 }}$ is shown. $\mathrm{n}=3$, SEM. Black, dark gray and light gray bars represent the samples containing the arrested $b_{2}(167)_{\Delta}$-DHFR in Tom $22^{\text {His10 }}$, not containing $\mathrm{b}_{2}(167)_{\Delta}$-DHFR in Tom $22^{\text {His10 }}$, and not containing $\mathrm{b}_{2}(167)_{\Delta}$-DHFR in WT, respectively.

\subsubsection{Different TIM23 or PAM mutants display contradicting phenotypes}

\subsubsection{TIM23 complex composition in mutants}

In order to test whether the exchange of TIM23 and PAM subunits into the active translocase is of mechanistic relevance, different mutants were analyzed. In addition to the temperature sensitive mutants described above, deletion mutants for PAM17, TIM21 and MGR2 were analyzed (Chacinska et al., 2005; Mokranjac et al., 2005a; van der Laan et al., 2005; Gebert et al., 2012).

Again $\alpha$-Tim23 co-immunoprecipitations, as described above, were used to assess the 
subunit composition in the different mutants. In pam17 $\Delta$ mutant mitochondria no alterations in the complex composition were observed (Figure 34.A). In contrast in tim21 $\Delta$ the steady state levels and consequently the amount of precipitated Mgr2 was decreased (Figure 34.B). Surprisingly, in the mgr2 $\Delta$ a residual Tim21 association with the translocase was found (Figure 34.C), even though Mgr2 was described as the protein required to coupled Tim21 to the TIM23 complex (Gebert et al., 2012).
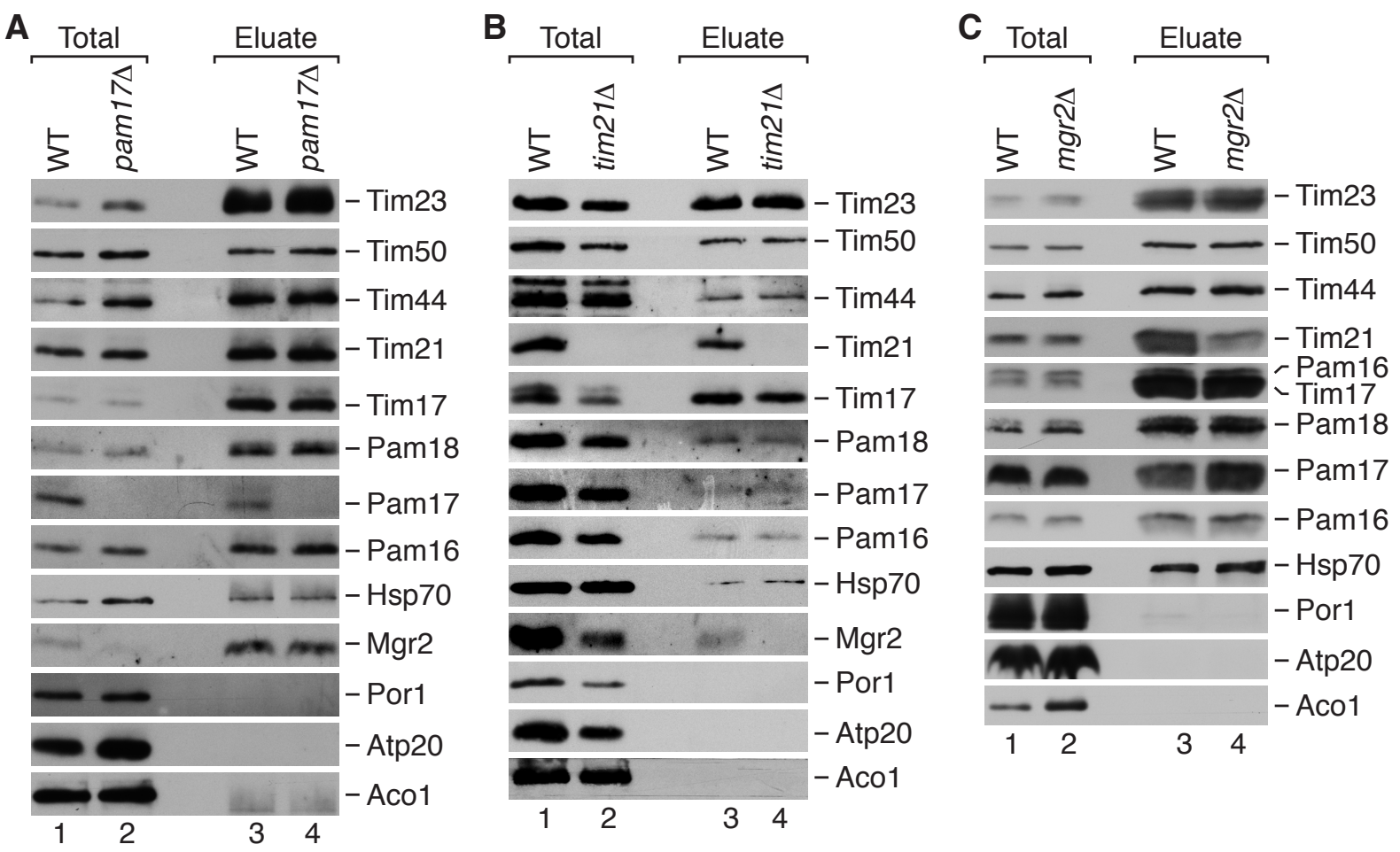

Fig. 34: Deletion mutants of TIM23 and PAM components reveal a normal composition of the TIM23 complex - Digitonin solubilized mitochondria were subjected to $\alpha$-Tim23 co-immunoprecipitations. Samples were analyzed by SDS-PAGE and western blotting. Total $5 \%$, eluate $100 \%$. A WT and pam17 . B WT and tim21 $\Delta$. C $\mathrm{WT}$ and $m g r 2 \Delta$.

\subsubsection{Import and inward driving activity in mutants}

In order to test the effect of the alterations in the subunit composition on the import efficiency the matrix targeted F1 $\beta$ was imported in vitro. Import into temperature sensitive mitochondria was performed after incubation for $15 \mathrm{~min}$ at $37^{\circ} \mathrm{C}$ to induce the phenotype (Figure 35.A). 
As a reference tim54-11 mitochondria were used, which are unaffected in presequence transport as Tim54 is important for import of carrier proteins (Wagner et al., 2008). Accordingly, import of F1 $\beta$ was at $100 \%$ of the respective WT. In ssc1-3 mitochondria import was abolished, as previously shown (Gambill et al., 1993). Both pam17s and mgr2 $\Delta$ showed a $70 \%$ decrease in matrix import compared to WT. As mentioned above, pam16-3 and pam18-66 mutant mitochondria imported F1 $\beta$ substrates only slightly less efficient compared to the respective WT, and similar to the tim54-11. Previously, van der Laan et al. (2005) showed that sorting of motor-independent precursor proteins is unaffected in pam17 $\Delta$. Similarly, the import of $b_{2}(167)$-DHFR as well as DLD-DHFR was not defective in mgro $\Delta$ (Figure 35.B).

In summary, the ssc1-3 allele showed the strongest matrix import defect, followed by pam17 $\Delta$ and mgr2 $\Delta$.

To test the inward driving activity of the import motor in the different mutants the following assay was performed. After incubation of the ts strains at restrictive temperature, the sorted precursor $b_{2}(220)$-DHFR was imported in the presence of MTX for 15 min. In contrast to $\mathrm{b}_{2}(167)$-DHFR, $\mathrm{b}_{2}(220)$-DHFR is motor dependent, due to the heme binding domain (residues 81-181) (Rassow et al., 1989; Geissler et al., 2001). Subsequently, the membrane potential was dissipated using valinomycin and the reactions were chased. After different times a part of the reaction was removed and optionally PK treated, to remove all precursors except the ones that are inserted in the TIM23 translocase and tightly opposed to the outer membrane (Figure 36.A) (van der Laan et al., 2005).

Similar to the matrix import defect in $m g r \Delta$ mutant mitochondria, the intermediate form of the precursor is not PK protected (Figure 36.B). In order to compare the different mutants with mgr2 $\Delta$ the same assay was performed with a $\Delta t$ of 5 min (Figure 36.C). In the tim54-11 mutant mitochondria no difference compared to the respective WT was observed, while all other mutants tested showed a reduction. pam17 $\Delta$, pam16-3 and pam18-66 showed a reduction to approx. 40-50\%. ssc1-3 mitochondria were not used for this assay due to the complete lack of import in these mitochondria (Figure 35.A and B).

In conclusion the mgr $\Delta$ mutant mitochondria are not defective in lateral release of precursor proteins into the inner membrane. However they display a defect in matrix import as well as the inward driving activity of a motor dependent sorted precursor. 

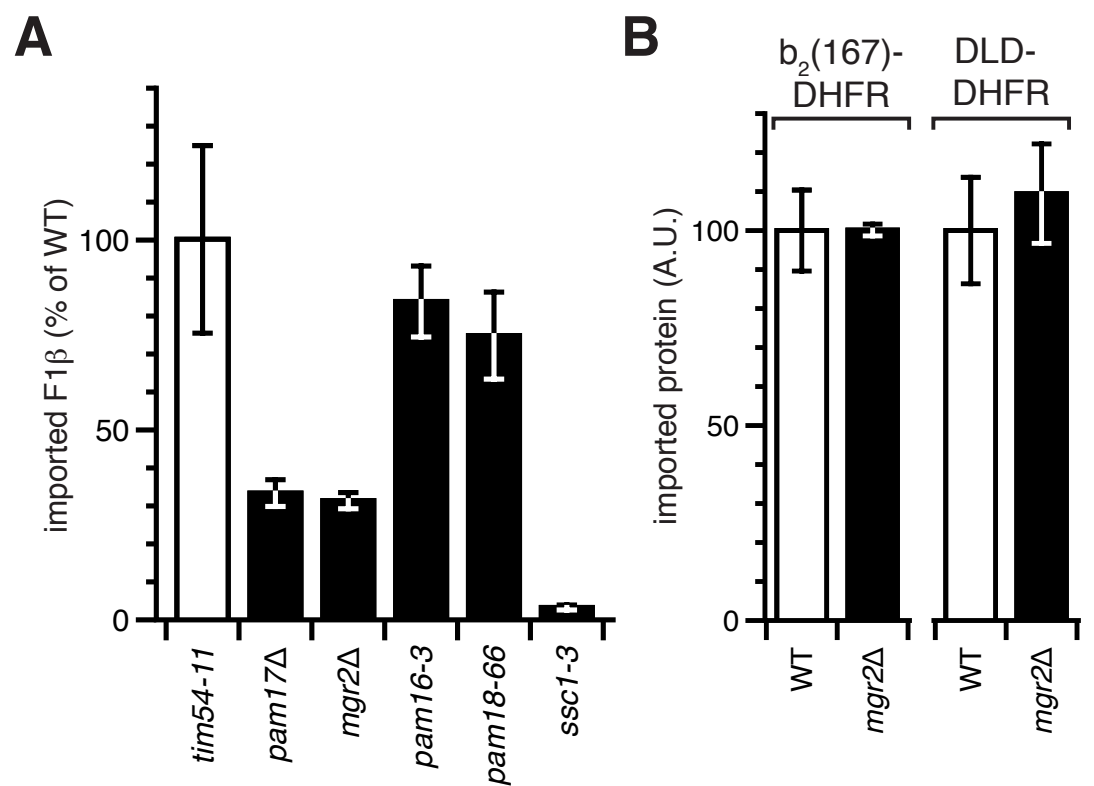

Fig. 35: TIM23 and PAM mutants display differential import phenotypes - A Import of radiolabeled $\mathrm{F} 1 \beta$ for $15 \mathrm{~min}$ into WT and the indicated mutant mitochondria. Temperature sensitive mutants were incubated for $15 \mathrm{~min}$ at $37^{\circ} \mathrm{C}$ before import. The reaction was stopped by the addition of AVO and PK treatment. Samples were analyzed by SDS-PAGE and autoradiography. Quantification of the imported mature protein was performed $(100 \%$ - import of respective WT, $\mathrm{n}=3$, SEM). B Import of the import motor independent, sorted $\mathrm{b}_{2}(167)$-DHFR and DLD-DHFR as described in A $(100 \%$ - import of respective WT, $n=3$, SEM).

\subsubsection{Efficient assembly of Pam18, but not Tim44, depends on Mgr2}

Even though all subunits are present at the translocase in pam17 $\Delta$ and mgr2 $\Delta$ mitochondria the import and inward driving activity was affected in both strains, even more severely than in the pam16 or pam18 temperature sensitive strains. This could be due to a reduced dynamics of subunits at the translocase. In order to test this, radiolabeled Pam18 was imported into WT and mgr2 $\Delta$ and integration into TIM23 was assayed by $\alpha$-Tim23 co-immunoprecipitations (Figure 37.A). A significant reduction by $\sim 40 \%$ of the assembly efficiency compared to WT was seen in the mgr2 $\Delta$ mutant mitochondria, while the integration of Tim44 was unaffected (Figure 37.B). Because the levels of Tim21 at the TIM23 complex are reduced in mgr2 $\Delta$ the assembly of Pam18 in tim21 $\Delta$ was also tested. Interestingly, the lack of Tim21 increased assembly efficiency of Pam18 to the TIM23 complex (Figure 37.B). 


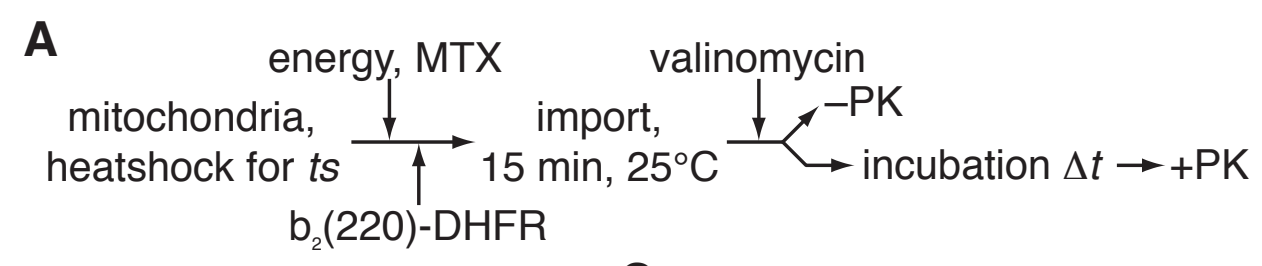

B
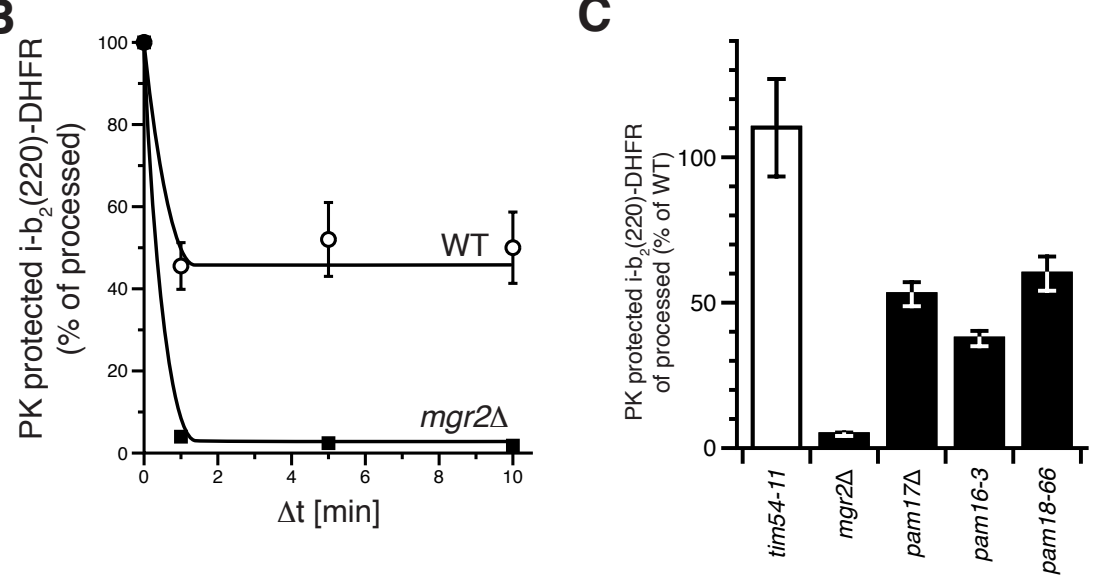

Fig. 36: TIM23 and PAM mutants display differential inward driving activity - A Scheme of the experimental setup assaying the inward driving activity. B Inward driving activity of $\mathrm{b}_{2}(220)_{\Delta}$-DHFR assayed in WT (circle) and $m g r \Delta$ (square) mitochondria with $\Delta \mathrm{t}=1,5,10$ min. Quantification as in $\mathrm{B}(\mathrm{n}=3$, SEM). C Inward driving activity assayed as in $\mathrm{B}(\Delta \mathrm{t}=5 \mathrm{~min}$ in different mutant mitochondria and their respective WT. Quantification as described in B (100\% - import of respective WT, $\mathrm{n}=3$, SEM).

This indicates that the dynamics of the subunit exchange in mgr2 $\Delta$ are slowed down, which is not visible at the level of subunit composition of the TIM23, but becomes evident during transport. Furthermore this supports the idea that the exchange of Pam18 is needed for the activity of the import motor. 
A

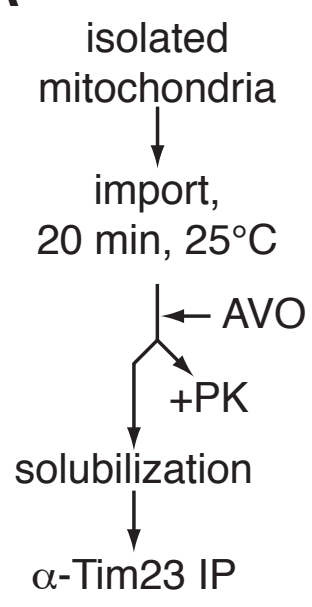

B

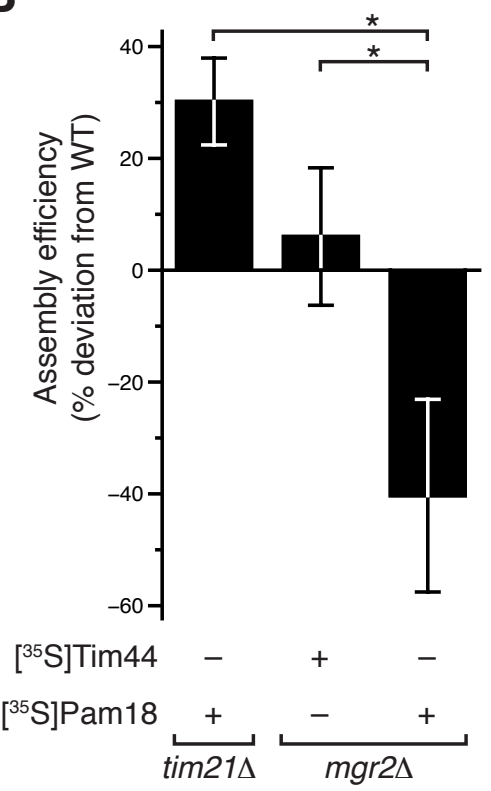

Fig. 37: Integration of Pam18 into TIM23 is impaired in mgr2 $\Delta$ - A Scheme of the experimental setup assaying the integration of radiolabeled Pam18 or Tim44 into the TIM23 complex in WT and mutant mitochondria using $\alpha$-Tim23 coimmunoprecipitations. B As described in A, radiolabeled Pam18 or Tim44 were imported into WT and tim21 $\Delta$ or mgr2 $\Delta$ mitochondria. Subsequently, the samples were subjected to $\alpha$-Tim23 co-immunoprecipitations. Samples were analyzed by SDS-PAGE, autoradiography and western blotting. Quantification of the assembly efficiency of the imported protein compared to WT is shown (as deviation from the respective WT, samples were normalized to the Tim23 and Pam18/ Tim44 IP efficiency, $\mathrm{n}=3$, SEM). 


\section{Discussion}

Protein transport into mitochondria and the sorting into the respective subcompartment is an essential process for eukaryotic cells. In the last decades the field of mitochondrial protein import advanced rapidly from knowing a single transport pathway (presequence pathway) (Schatz and Butow, 1983), to the current model of at least five different pathways (Dudek et al., 2013). The identification of new import pathways was paralleled by the identification of new protein subunits involved in protein translocation. Now we know e.g. five subunits of the TIM23 complex and seven subunits of the import motor (PAM). Some of these subunits like the Mgr2 were only recently identified (Gebert et al., 2012), highlighting the dynamics of the field. Although recent proteomic studies employed sophisticated quantitative mass spectrometric approaches, we can not be completely certain that all subunits of the translocase complexes are identified.

In contrast to the increasing information about the different translocase subunits, our knowledge about the recognition of the targeting signals remained static. The best and only information is the interaction between the presequence and the receptor Tom20. Abe et al. (2000) provided an atomic model of this interaction, clearly showing the recognition of the hydrophobic side of the presequence helix (Figure 4.B). In the first part of this study I utilized presequence photopeptides to identify new presequence receptors. In the second part, moving with the precursor past the recognition stage and across the inner membrane, the regulation of the import motor was studied.

\subsection{Presequence photopeptides as a tool to identify presequence receptors}

Since the early days of mitochondrial protein translocation research, synthetic presequence peptides have been used to define the properties of a functional presequence (Gillespie et al., 1985; Allison and Schatz, 1986; von Heijne, 1986). In addition to the use of peptides, different crosslinking approaches have been widely used. These range from using unspecific crosslinkers (Popov-Celeketić et al., 2011), cysteine mutants in combination with 
thiol-specific crosslinkers (Alder et al., 2008b; Lytovchenko et al., 2013), the coupling of functional probes to specific sites in a protein (Alder et al., 2008a), to the incorporation of benzophenylalanine into in vitro synthesized precursor proteins (Kanamori et al., 1997; Shiota et al., 2011). All of these approaches helped to define interactions between the substrate and the translocase as well as between translocase components. One major drawback of most of these methods is that they do not allow the identification of the amino acid that has been crosslinked on the target protein.

A solution to this problem is the use of chemically synthesized peptides with incorporated crosslinking groups that allow dissection of the photoadduct by mass spectrometry. One chemical group that has been successfully used for this is benzophenylalanine (BPA) (Prestwich et al., 1997; Jahn et al., 2004; Dimova et al., 2006). In this study we used a similar approach: BPA was incorporated into peptides based on the aldehydedehydrogenase presequence from rat. Versatility was further increased by an additional biotinyllysine as well as a His6 tag.

These modifications of the photopeptides $\mathrm{pS}_{16} \mathrm{~B}$ and $\mathrm{pL}_{19} \mathrm{~B}$ did not impair their potency as a targeting signal, as they imported efficiently into the mitochondrial matrix in a membrane potential-dependent manner following the presequence pathway. The resilience of the import machinery to bulky or branched substrates has been reported previously (Vestweber and Schatz, 1988). While some studies showed deleterious effects of the amphipathic presequence peptides (Gillespie et al., 1985; Roise et al., 1988), the presequence photopeptides did not have a negative effect on the integrity of the mitochondria membranes, as the membrane potential and import of TIM22 substrates were not affected (Figure 24) (Schulz et al., 2011). Furthermore, the competition of presequence import could simply be reversed by washing the mitochondria to remove the peptides, indicating structural integrity of the inner mitochondrial membrane.

I took advantage of the fact that the photopeptides are rapidly transported into the matrix along the presequence pathway, to crosslink them in transit to their receptors. Subsequent purification increased the sensitivity and allowed for the identification of more crosslinking partners. Although in this study a candidate approach was used, in the future an unbiased approach could also be envisioned. This would use photopeptides based on an authentic presequence as well as a control sequence (e.g. pCox4 and SynB2 or pALDH and pALDH-s). Photocrosslinking and purification of the crosslink adducts could be combined with a stable isotope labeling approach (SILAC) and quantitative mass spectrometry to 
identify novel presequence receptors.

Among the known candidates tested were the TOM receptors Tom20 and Tom22. Some previously published studies failed to generate in organello crosslinks between the presequence and the receptors of the TOM complex on the cis side (Esaki et al., 2004; Yamamoto et al., 2011) while others succeeded. Gillespie (1987) generated an in organello presequence peptide crosslink to Tom20 or Tom22 and Rapaport et al. (1997) showed a crosslink with Tom22 in outer membrane vesicles. Similarly, I identified in organello photocrosslinks to the presequence receptors Tom20 and Tom22. As Tom20 only contains a TMD and the cytosolic domain, the photoadduct reflects the known recognition of the presequence by this domain (Abe et al., 2000; Schulz et al., 2011). The crosslinking efficiency was better with $\mathrm{pL}_{19} \mathrm{~B}$, which contains the BPA on the hydrophobic side of the amphipathic presequence helix, in line with the known hydrophobic interaction between Tom20 and the presequence (Figure 13.A). However, Tom22 showed a similar trend (Figure 14.A), although it is established that in vitro Tom22 recognizes the hydrophilic side of the presequence helix (Brix et al., 1997). This could be explained by (a) the fact that Tom20 and Tom22 form a composite receptor in organello (Shiota et al., 2011) or (b) by the variable reaction radius of the BPA ranging from 3.1-14 $\AA$ (Dormán and Prestwich, 2000; Wittelsberger et al., 2006). This range might allow to crosslink the BPA to a single receptor that only recognizes one side of the helix even though the BPA is facing outwards. Based on the Tom20 structure this would be possible (Figure 38), at the same time explaining why photoadducts between Tom20 and $\mathrm{pS}_{16} \mathrm{~B}$ were observed. The photoadducts to Tom22 could also be located at the intermembrane space domain. This could be tested in the future by comparing the crosslinking pattern in wild-type and tom22-2 mitochondria lacking the IMS domain (Moczko et al., 1997).

When we identified the residues crosslinked to the cytosolic domain of Tom20 and mapped them on the model structure of yeast Tom20, based on the Tom20-pALDH structure, we found that the crosslinked residues were within the $14 \AA$ limit (Schulz et al., 2011). Hence, it is feasible to determine presequence binding sites with this approach.

Apart from the crosslinks to the classical presequence receptors of the TOM complex, adducts to the channel formed by Tom40 were found. This result is in line with previous work (Gaikwad and Cumsky, 1994; Rapaport et al., 1997). The stage of crosslink generation with Tom40 is still unknown: it could occur on the cis site, upon passage through or exit from the channel at the trans site. As presequence peptides have been shown to 


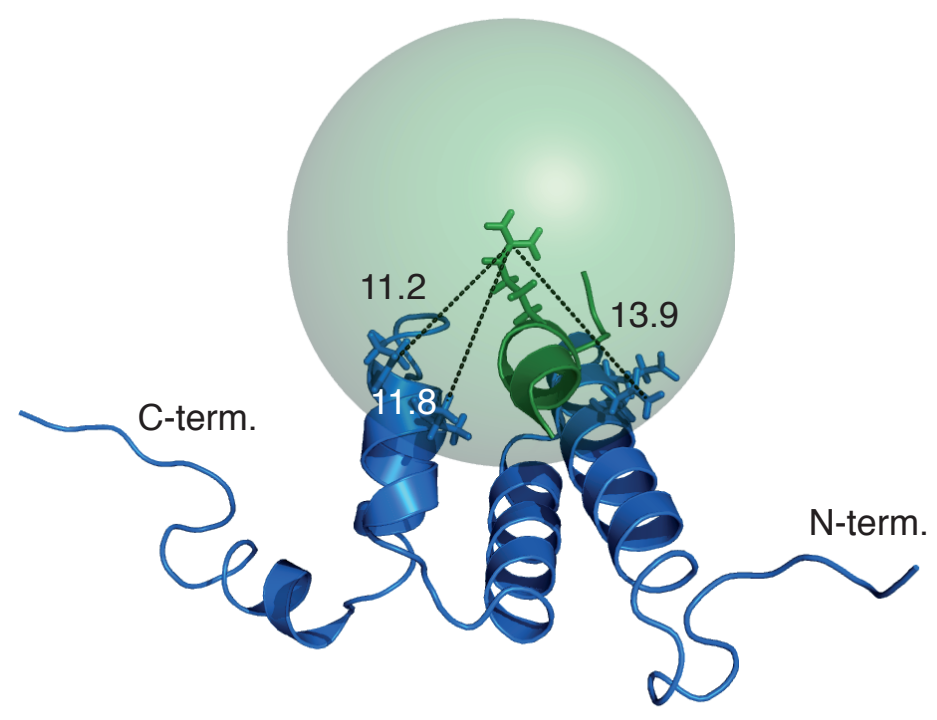

Fig. 38: Distances between Tom20 and pALDH - Cartoon representation of the NMR structure of Tom20 (blue) and pALDH (green) (PDB ID: 1OM2, Abe et al. (2000)). Several distances (in $\AA$ ) between the outward facing arginine of pALDH to residues of Tom20 are displayed. The green sphere corresponds to a radius of $14 \AA$.

influence gating of the Tom40 channel (Hill et al., 1998), the photoadducts might reflect an interaction on the cis side required for this regulation.

Interestingly, photoadducts were also generated with the internal targeting signal receptor Tom70. Previous studied indicated that Tom70 binds presequences (Komiya et al., 1997). However, this was debated (Brix et al., 1997). The current hypothesis is that Tom70 recognizes the hydrophobic mature part of some presequence containing precursor proteins (Yamamoto et al., 2009). Our results shed a new light on this controversy as Tom70 might indeed be involved in the recognition of the presequence. Clearly, the presequence interaction of both Tom40 and Tom70 require more detailed investigations using e.g. mutants in order to provide insight into the physiological importance.

In addition to the crosslinks at the TOM complex, the photopeptides also engaged in adducts with TIM23 subunits. A crosslink to Tim21 indicated that it might play a role as a presequence receptor in the presequence translocase. However, we could recently show that Tim21 interacts with control peptides as well, hence displaying an unspecific peptide binding capacity (Lytovchenko et al., 2013). The photoadduct with Tim23 could represent the validated presequence recognition by the intrinsically disordered IMS domain of Tim23 (Komiya et al., 1998; Gevorkyan-Airapetov et al., 2009). De la Cruz et al. (2010) 
determined the affinity for the interaction between Tim $23^{\mathrm{IMS}}$ and pADLH to be $K_{\mathrm{D}}=$ $0.47 \mathrm{mM}$.

No photoadduct was detectable in organello for Tim44, although Marom et al. (2011) recently showed a high affinity of Tim44 for presequence peptides in vitro $\left(K_{\mathrm{D}}=230 \mathrm{nM}\right)$. This could be due to a rapid clearance of the presequence from the exit site of the TIM23 translocase by an unknown mechanism. Rapid degradation in the matrix by e.g. PreP seems unlikely as the peptides were observed in the matrix after import (Teixeira and Glaser, 2013). However, a more likely explanation is that the in organello crosslinking was performed on ice without the supply of ATP or NADH to establish and maintain the $\Delta \psi$. Hence, although isolated mitochondria usually retain a small $\Delta \psi$, this might not be enough to support translocation of enough photopeptides across the inner membrane at $4{ }^{\circ} \mathrm{C}$ in order to generate detectable amounts of a Tim44 photoadduct. Further experiments should take this into account in order to determine whether a presequence adduct to Tim44 can be generated in organello. Additionally if crosslinking sites could be mapped using recombinant Tim44, the available structure can be used to aid the generation of mutants.

In addition to these crosslinks I found a photoadduct between $\mathrm{pL}_{19} \mathrm{~B} / \mathrm{pS}_{16} \mathrm{~B}$ and Tim50 (Schulz et al., 2011). At the same time Yamamoto et al. (2011) found a crosslink between Tim50 and the N-terminal part of an arrested precursor and Marom et al. (2011) measured the affinity of purified Tim $50^{\mathrm{IMS}}$ to presequence peptides to be $K_{\mathrm{D}}=45 \mu \mathrm{M}$. We measured a ten fold lower affinity, however using a different targeting signal (Lytovchenko et al., 2013). In conlusion, three different groups confirmed presequence binding to Tim50, however only now with the photocrosslink a unique opportunity to identify the binding site on Tim50 became available. 


\subsection{Tim50 - an essential presequence receptor of the TIM23 complex}

\subsubsection{Tim50 contains an essential C-terminal presequence bind- ing domain}

A study by Mokranjac et al. (2009) showed that in organello the function of Tim50 as a precursor receptor depends on its association with the TIM23 complex, especially Tim23. However, here I could show that in vitro Tim50 $0^{\mathrm{IMS}}$ can interact with presequence peptides independently. This was later supported by the same group (Marom et al., 2011). Additionally, I could show efficient presequence recognition by Tim50 in organello in mitochondria depleted of Tim23 (Figure 22.C, E and F). Hence, presequence recognition by Tim50 functions independent of Tim23, while precursor binding at a later stage requires $\operatorname{Tim} 23$.

The advantage of the Tim50 5 IMS photoadduct generated in vitro was that a crosslink in preparative scale allowed us to identify the C-terminal presequence binding domain (residues 395-476) (Figure 15.A and C). In vitro the PBD can bind to presequence peptides independent of the rest of the IMS domain. The interaction of the PBD with presequence peptides appears to be a hydrophobic interaction (Figure 16.D). However, as shown for $\operatorname{Tim} 50^{\mathrm{C}}$, crosslinking efficiencies and affinities do not necessarily correlate with each other. Therefore the type of interaction should be verified using an independent approach, e.g. SPR under different conditions (Lytovchenko et al., 2013).

The PBD forms a separate fold and is connected to the remaining IMS domain by a flexible and partly hydrophobic linker (Figure 15.C and 16.B). According to secondary structure predictions the PBD is formed by two $\alpha$-helices that are connected by loops, which might form a similar fold like Tom20 (Figure 4.B). However, although substantial amounts of PBD can be produced, structural studies have so far not yielded any atomic insight into the organization of the domain in order to test this hypothesis. If the generation of Tim50 $\mathrm{PBD}$ crystals can not be accomplished, the structure could also be determined by NMR as the domain is only $10 \mathrm{kDa}$.

Surprisingly, quantitative assessment of the affinities of the different Tim50 constructs revealed that $\operatorname{Tim}_{50} \mathrm{C}$, a more stable version of Tim50 ${ }^{\triangle \mathrm{PBD}}$, showed a specific affinity 
to presequence peptides which was comparable to Tim50 ${ }^{\mathrm{IMS}}$ (Table 4). Additionally, chemical crosslinking of Tim50 $\triangle \mathrm{PBD}$ to presequence peptides revealed small amount of adduct (Schulz et al., 2011). We identified the second binding site in Tim50 ${ }^{\mathrm{C}}$ in proximity to the negatively charged groove. This groove was proposed as a presequence binding site by Qian et al. (2011). In the future mutants will be required to determine the mechanistic contribution of this second binding site to the import process in organello as it does not support cell viability in the absence of the PBD.

Even though Tim50 ${ }^{\mathrm{IMS}}$ seems to contain two presequence binding sites, the SPR data of the Tim $50^{\text {IMS }}$ presequence interaction indicates a single binding site (Lytovchenko et al., 2013). Independent binding to both sites or a sequential binding mechanism therefore appear to be unlikely. It rather seems that, although both the PBD and NIF domain form stable independent folds, they constitute a composite binding site similar to that formed by Tom20 and Tom22 (Shiota et al., 2011). It could be expected that both domains recognize opposite surfaces of the presequence helix, e.g. the negatively charged groove binds to the hydrophilic side, while the PBD binds to the hydrophobic site (Figure 16.D) (Qian et al., 2011). From this model one would anticipate a drop in affinity when a part of the binding site is missing. This was actually observed for the PBD, which showed an approximately two fold lower affinity compared to Tim50 ${ }^{\mathrm{IMS}}$ and $\mathrm{Tim}^{\mathrm{C}} \mathrm{C}^{\mathrm{C}}$ (Table 4).

Alternatively, only one binding site might be accessible during the SPR measurements, comparing the affinities this could be the one in proximity to the negatively charged groove. Although the PBD seems to be independent of the remaining IMS domain, it can't be excluded that this part sterically interferes with presequence recognition by the PBD. Due to the importance of the PBD for cell viability and protein import one can speculate that this effect might only occur in vitro and is prevented in vivo due to the interplay with another subunit, for example Tim21 or Tim23.

Further studies will be required to investigate the detailed spatial and functional relationship between both binding sites. These studies could for example be based on fluorescence resonance energy transfer (FRET) with the PBD and the core domain labeled in order to monitor the relative changes of both domains upon presequence addition. A similiar approach was used by Mapa et al. (2010) to investigate the conformational changes of $\operatorname{mtHsp} 70$.

Although the PBD of Tim50 is conserved in fungi (Figure 16.A), in higher eukaryotes there are some major differences (Figure 39.A). First, the PBD is not conserved between 
the human and yeast Tim50. Only a few residues corresponding to the yeast residues 420-450 are identical or similar. Second, the linker between the putative PBD and the core of the IMS domain is very short. Third, the protein is in general smaller due to deletions of several segments. Fourth, the negatively charged groove found in the yeast structure appears to be uncharged in the human structure (Figure 39.B). Hence, presequence recognition by H.s. Tim50 might employ a different mechanism. One possibility could be a domain swap with one of the other TIM23 components, but none of the known human TIM23 proteins contains a significant extension in the IMS (data not shown). Alternatively, presequence recognition might involve hydrophobic instead of hydrophilic interactions. However, no significant hydrophobic cluster can be found on the surface of the H.s. Tim50 model. Additionally, Zhang et al. (2012a) reported binding of divalent ions to H.s. Tim50 IMS and an influence of these ions on the presequence recognition. This recognition was shown to be largely mediated by electrostatic interactions (Zhang et al., 2012b). Clearly the investigation of the presequence recognition by H.s. TIM23 will be interesting and might reveal a different mechanism compared to the one observed in yeast.

\subsubsection{Presequence recognition by Tim50s $\mathrm{PBD}$ is needed for transport across the inner membrane}

The presequence binding domain is essential for cell viability in yeast. This is not due to a lack of Tim50 in TIM23, as the association of Tim50 ${ }^{\triangle \mathrm{PBD}}$ with the TIM23 complex was comparable to WT. Together with the in vitro pulldown using Tim23 ${ }^{\mathrm{IMS}}$ this shows that Tim50 binding to Tim23 is largely mediated by the TMD (Alder et al., 2008b) and the core domain with only minor contributions of the PBD.

Deletion of the domain impairs protein transport by the TIM23 complex across the inner mitochondrial membrane. Tim50 has been recently shown to be in close proximity to precursors arrested in the TOM complex (Yamamoto et al., 2011). The removal of the PBD does not affect the establishment of this TOM intermediate (Figure 23.C). In mitoplasts (mitochondria with an osmotically opened outer membrane), when presequence recognition by the $\mathrm{PBD}$ is perturbed by antibodies or deletion of the domain, protein import is impaired, indicating that the presence of the presequence binding groove in $\operatorname{Tim} 50^{\triangle \mathrm{PBD}}$ is not sufficient to mediate import across the inner membrane.

In addition to the receptor function, Tim50 is also involved in closing the Tim23 

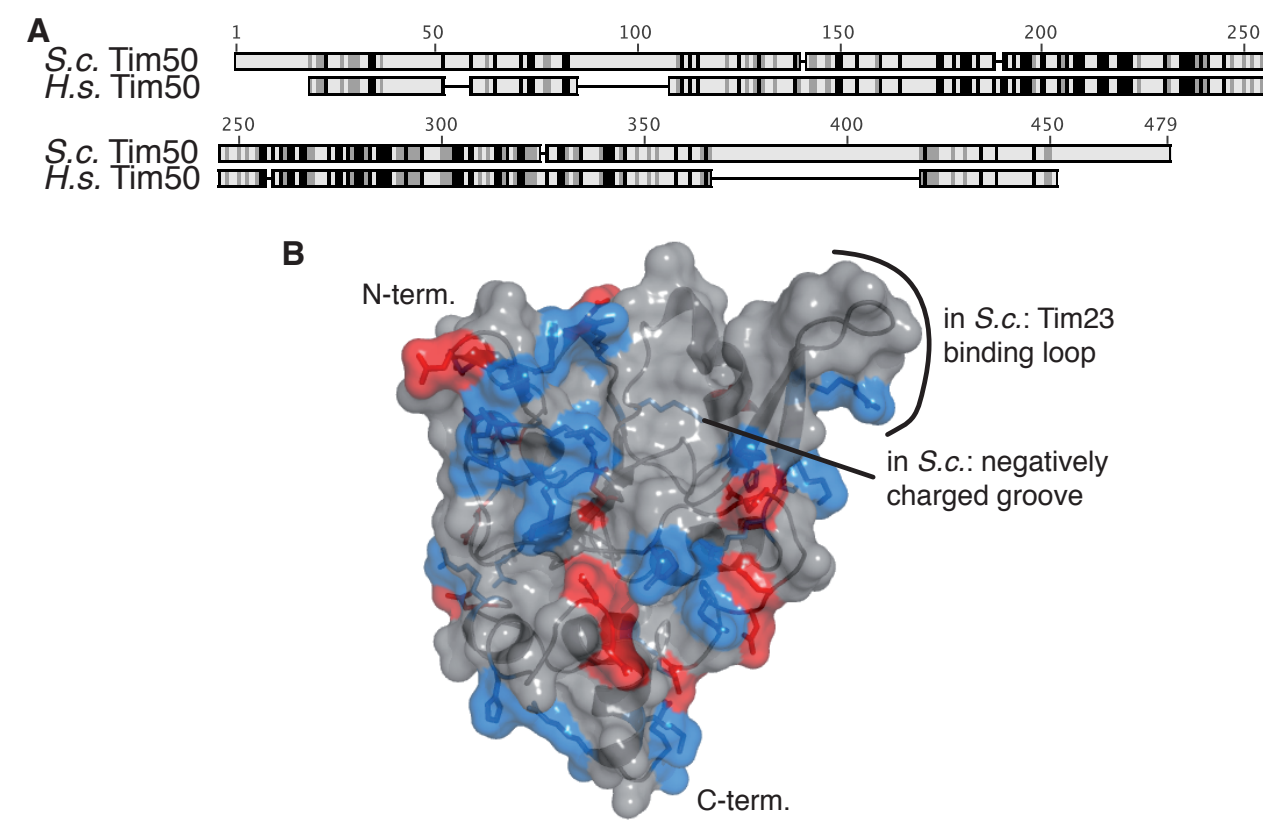

Fig. 39: Comparison between Saccharomyces cerevisiae and Homo sapiens Tim50

- A ClustalW alignment of S.c. and H.s. Tim50. Identical, similar and not similar residues are colored in black, dark gray and light gray, respectively. B Swissmodel workspace model of H.s. Tim50 based on the yeast structure (PDB ID: 3QLE) (Arnold et al., 2006; Qian et al., 2011). The position of the Tim23 binding loop as well as the negatively charged presequence binding groove found in S.c. are indicated. Positively and negatively charged residues are colored in blue and red, respectively.

channel (Meinecke et al., 2006; Alder et al., 2008b). It seems that the PBD is not involved in this process, as the mitochondrial membrane potential in the Tim50 $\downarrow+\operatorname{Tim} 50^{\triangle \mathrm{PBD}-\mathrm{HA}}$ mitochondria is similar to the respective WT (Figure 24). Additionally, $\Delta \psi$ dependent import along the TIM22 pathway was increased in the mutant, an observation that has also been made by others when the TIM23 or PAM complexes were compromised (Geissler et al., 2002; Yamamoto et al., 2002; Frazier et al., 2004; Schulz et al., 2011). However, it can not be excluded that the small amounts of Tim50 ${ }^{\mathrm{WT}}$ retained after shut down are sufficient to mediate the closure of Tim23 and therefore prevent $\Delta \psi$ leakage. This remaining fraction of Tim50 most likely also accounts for the residual $25 \%$ of import.

These results suggest that Tim50 is the primary presequence receptor of the TIM23 complex for the following reasons: Presequence binding to the IMS domain of Tim23 is not sufficient for transport across the inner membrane, most likely due to the low affinity of the interaction. Additionally, the presequence recognition by Tim $21^{\text {IMS }}$ is unspecific 
and Tim21 itself is not essential. Of the other known TIM23/ PAM subunits only Pam18 extends a domain into the IMS. However, this N-terminal part is mainly involved in recruitment of Pam18 to Tim17, and its deletion produces no import or growth phenotype (Mokranjac et al., 2007; D'Silva et al., 2008; Schilke and Craig, 2011). Furthermore, the motor free sorting form of TIM23 is thought to be the default state of the translocase that engages the emerging precursor due to the coupling of the TOM and TIM23 complexes by Tim50, Tim21 and Tom22 (Chacinska et al., 2005; Albrecht et al., 2006; Tamura et al., 2009; Dudek et al., 2013; Lytovchenko et al., 2013).

The IMS domain of Tom22 is widely accepted to be another presequence binding site in the intermembrane space based on a competition of precursor binding to Tom $22^{\text {IMS }}$ by presequence peptides (Komiya et al., 1998; Chacinska et al., 2005). Deletion of the domain causes a slight growth defect at $37^{\circ} \mathrm{C}$ as well as slight import defects of presequence containing precursor proteins (Moczko et al., 1997). Interestingly, the domain is especially important for a two-step import where the $\Delta \psi$ is generated only in the second step, pointing to a potential back-up mechanism that enables new import attempts when the presequence slid back from the TIM23 complex in the first place.

Hence, Tim50 with its PBD is the primary presequence receptor of the TIM23 translocase and is essential for efficient precursor transport across the inner membrane.

\subsubsection{Handover is coordinated in a trimeric complex between the presequence, Tim23 and Tim50}

As both Tim50 and Tim23 interact with the presequence in the intermembrane space the functional relationship between both binding sites was especially interesting (de la Cruz et al., 2010; Schulz et al., 2011; Marom et al., 2011; Lytovchenko et al., 2013). While late stages of precursor binding by Tim50 are Tim23-dependent (Mokranjac et al., 2009), I could show that presequence binding is independent of Tim23. In line with that, overexpression of Tim50 increased the generation of the photopeptide-Tim50 adduct and matrix import. In vitro titrations allowed us to deduce that Tim50 and presequence binding to Tim23 are mutually exclusive (Schulz et al., 2011). Furthermore I could generate a trimeric complex containing the presequence, Tim50 and Tim23 (Figure 25). A similar complex was also observed by Marom et al. (2011) using a Hsp60 presequence peptide. This complex most likely contains a Tim23 bound to Tim50s Tim23-binding $\beta$-loop and 
the presequence bound to the presequence binding domain or groove of Tim50 (Schulz et al., 2011; Qian et al., 2011). As for the dynamics within Tim50, it will be interesting to investigate details of the presequence transfer from Tim50 to Tim23 using more detailed methods.

\subsubsection{Model of early transport steps by the TIM23 translocase}

The model of precursor recognition and transport through the intermembrane space can be updated in the following way (Figure 40). Tim50 is the primary presequence receptor of the TIM23 in the IMS (A). Presequence recognition by Tim50 (B, using the PBD or a composite binding site containing the PBD and the negatively charged groove) triggers release of Tim21 from Tim50 in a Tim23 dependent manner (C-D) (Lytovchenko et al., 2013). The Tim $50^{\mathrm{IMS}}$-presequence complex binds to Tim $23^{\mathrm{IMS}}$. After transfer of the presequence to Tim $23^{\mathrm{IMS}}$, contacts between Tim $23^{\mathrm{IMS}}$ and Tim $50^{\mathrm{IMS}}$ are lost (E). Due to the removal of Tim21, Pam17 is recruited, which initiates assembly of the import motor (E). The presequence is then passed to the TIM23 pore $(\mathrm{F})$. The binding of the targeting signal by Tom $22^{\mathrm{IMS}}$ might be a back-up mechanism to prevent backsliding $(\mathrm{G})$.
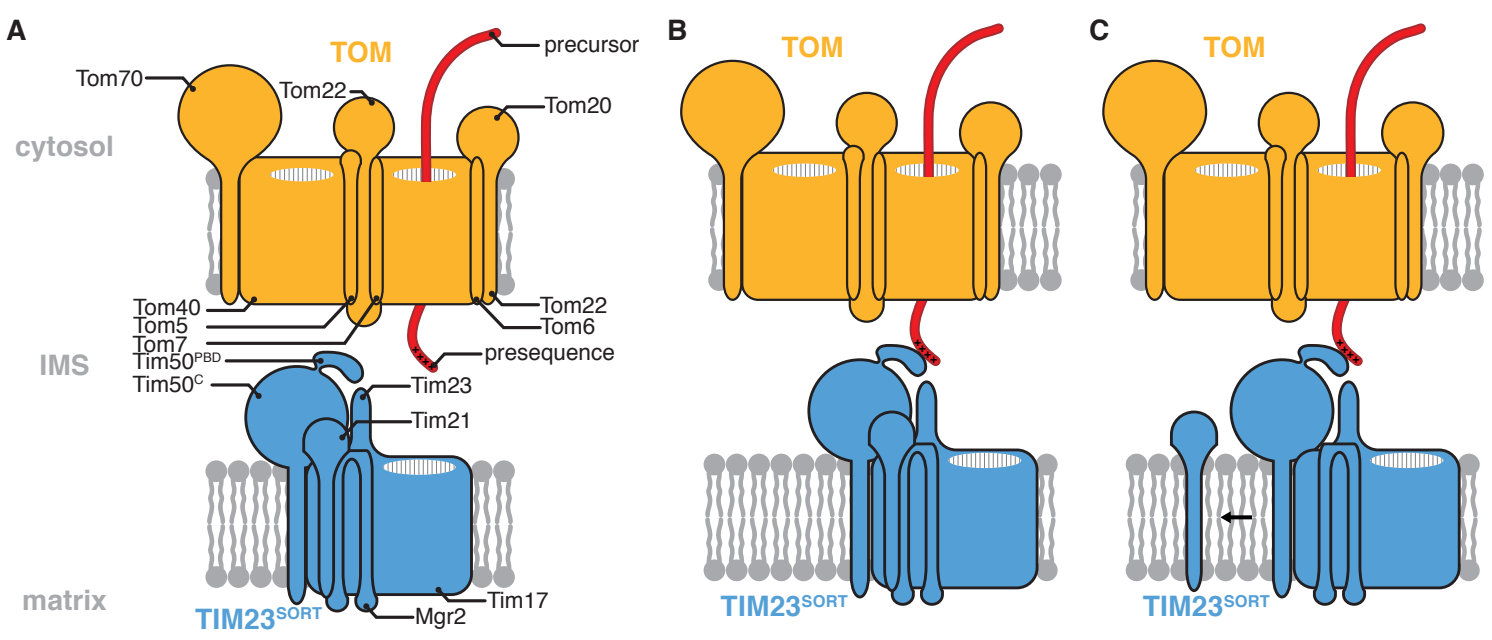

Fig. 40: Presequence recognition and precursor transport in the IMS - A After emergence from the TOM channel the presequence reaches the intermembrane space. The default state of the TIM23 is the Tim 21 containing sorting form. B The presequence binding domain of Tim50 binds the presequence. C Tim21 is released from Tim50, enabling it to exit the TIM23 complex. (Continued on next page.) 


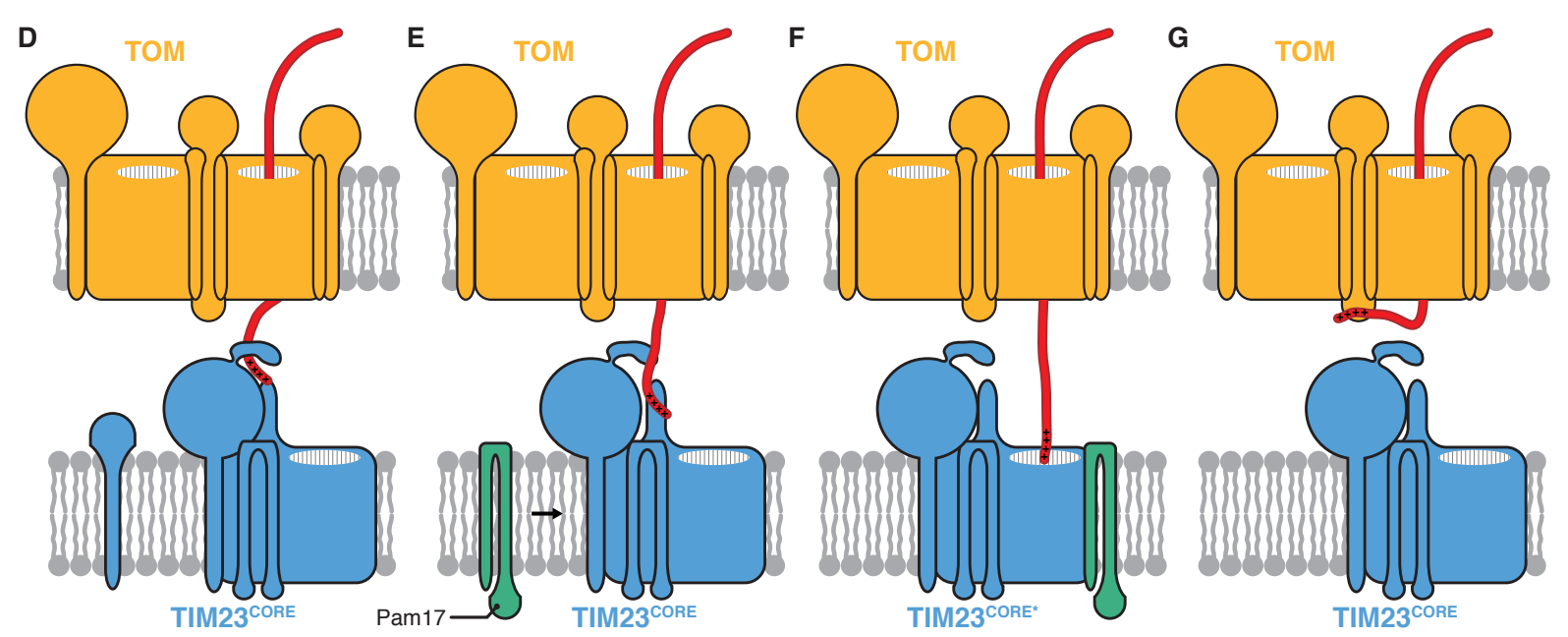

Fig. 40: (Continued from previous page.) - D Presequence recognition by Tim50 might involve a composite binding site using the PBD as well as the negatively charged groove in the core domain. The Tim $50^{\mathrm{IMS}}$-presequence complex binds to Tim $23^{\mathrm{IMS}}$. E Transfer of the presequence to Tim $23^{\text {IMS }}$, the contact between $\operatorname{Tim} 23^{\text {IMS }}$ and Tim $50^{\text {IMS }}$ is lost. Triggered by the release of Tim21, Pam17 is recruited to TIM23 enabling subsequent assembly of PAM. F From Tim $23^{\mathrm{IMS}}$ the presequence continues to the channel of the TIM23 complex. G A back-up mechanism to prevent backsliding of the precursor might involve presequence binding to Tom $22^{\mathrm{IMS}}$. 


\subsection{Subunit oscillation in the active translocase}

The import motor with its central subunit Hsp70 is a multi subunit chaperone system. The spatial and temporal regulation of Hsp70 activity is needed in order to generate vectorial movement of the precursor into the matrix. According to the molecular ratchet and entropic pulling model, Hsp70 undergoes multiple rounds of recruitment to the translocase. Binding of the substrate and detachment from the translocase, lead to a cycling of Hsp70 molecules during import. In order to analyze whether the regulation of Hsp70 is linked to this cycle I addressed the exchange of PAM and TIM23 subunits into the active translocase.

\subsubsection{In vitro import and assembly of TIM23 and PAM sub- units}

A prerequisite was to isolate all relevant subunits with the translocase after they were imported as radiolabeled precursors. As shown before all translocase associated TIM23 (Tim50, Tim23, Tim17, Tim21, Mgr2) and PAM (Tim44, Pam18, Pam17, Pam16) subunits were successfully co-isolated using Tim $23^{\mathrm{PA}}$ (Figure 26.A). Previous studies reported the isolation of the unlabeled proteins under similar conditions (Geissler et al., 2002; Truscott et al., 2003; Frazier et al., 2004; Chacinska et al., 2005; van der Laan et al., 2005; Gebert et al., 2012). The low association of Hsp70 (Ssc1) is in agreement with previous reports (van der Laan et al., 2005). For Tim23 the low co-isolation efficiency can be explained by the Tim $23^{\mathrm{PA}}$ overexpression. If the stoichiometry of Tim23 in TIM23 was one per complex, only complexes containing the protein A tagged version would be isolated. Most likely more than one copy is present in TIM23 and the stoichiometry might also be dynamic, especially taking the interplay with Tim17 into account. The other proteins are split into two groups, one of low co-isolation efficiency (Tim50, Tim44, Pam16, Pam18, Mgr2) and one of high (Tim21, Tim17, Pam17). A high efficiency indicates that the folding of the subunit after its import as well as the assembly from the free pool to the translocase-bound pool is fast. For all subunits except Tim23 and Tim17 this could be facilitated by the spatial proximity of the newly imported subunit the the TIM23 complex. For the subunits with a low efficiency one or both of these steps are slower. Alternatively the free pool might also be larger, hence making it less likely for an imported subunit to 
be bound to the translocase.

Except for Tim23 and Tim17, assembly of the other TIM23 and PAM subunits into the translocase has not been reported so far (Dekker et al., 1997; van der Laan et al., 2007). Hence, this system will provide a valuable tool to study the assembly of the TIM23 and PAM complex in the future as it can also be used in a tag-free system using $\alpha$-Tim23 co-immunoprecipitations (Figure 37.B).

The analysis of the integration of subunits into the active translocase relied on purified $\mathrm{b}_{2}(167)_{\Delta}$-DHFR as a matrix targeted import motor dependent substrate. Import in the presence of methotrexate induced a stable fold in the C-terminal DHFR domain, preventing passage through the TOM complex. The two membrane spanning intermediate generated in this way withstands biochemical procedures like affinity isolations (Figure 28.B (Rassow et al., 1989; Dekker et al., 1997; Popov-Celeketić et al., 2008a; Chacinska et al., 2010; Popov-Celeketić et al., 2011). The arrest of the precursor can be performed with amounts that lead to a $50 \%$ reduction of import of a reporter, which still allowed efficient co-purification of TIM23 (Figure 27.C and 28.C).

\subsubsection{The TOM-TIM23 supercomplex depends on the import motor}

Even though early studies by Dekker et al. (1997) showed that Hsp70 and Tim44 are not components of the TOM-TIM23 supercomplex on BN-PAGE, this was later corrected by several groups using mild affinity isolations (Popov-Celeketić et al., 2008a; Chacinska et al., 2010). The way the $\mathrm{b}_{2}(167)_{\Delta}$-DHFR is arrested across both membranes can be envisioned in two ways: The TIM $23^{\text {MOTOR }}$ complex could bind the precursor tightly and hence prevents backsliding. This might be supported by interactions of the TOM complex with the precursor. The generation of saturating amounts of the intermediate require the precursor not to slide back. If the arrested precursor would slide back, it can not be imported again, as the presequence is already cleaved off. This would diminish the yield of the translocation intermediate. The second possibility would be, that the import motor continuously tries to import the protein and thereby keeps the DHFR domain closely opposed to the outer membrane.

Using the temperature sensitive mutants ssc1-2 and ssc1-3 I could show that the initial generation of the intermediate requires Hsp70 function in order to generate a vectorial 
movement during import (Figure 29). Gambill et al. (1993) showed that ssc1-2 is still able to bind to nucleotides as well as precursor proteins during the import but lacks the ability to bind to Tim44. Hence the inefficient recruitment to the translocase exit site explains the inefficient matrix import (Kang et al., 1990; von Ahsen et al., 1995). ssc1-3 also shows a low matrix import efficiency (Figure 35.A), this is however due to an inability to bind to nucleotides rendering it non-functional (Gambill et al., 1993; Voos et al., 1996). The mutation of mtHsp70 in ssc1-3 does not affect the association of other TIM23 and PAM subunits with the translocase (Figure 32.A).

Furthermore, using ssc1-3, tim44-804 and pam18-66 I could show, that the ATPase activity of Hsp70 is needed to maintain a stable supercomplex. In tim44-804 matrix import is reduced, however lateral sorting is not affected. The mutant version of Tim44 does not recruit the Pam16/ Pam18 complex but retains higher levels of Pam17 in close proximity to Tim23 (Hutu et al., 2008). Similarly Truscott et al. (2003) showed that pam18-66 leads to a reduced matrix import due to a more labile association of Hsp70 with Tim44 and reduced levels of Pam18 at the translocase (Figure 32.B).

Hence, although other interactions of the precursor with the translocase machineries are also required, the import motor (Hsp70) activity is required for the generation and stabilization of the supercomplex. Similar results have been obtained with different precursor proteins (Ungermann et al., 1994). Therefore, it appears that the import motor is active when the $\mathrm{b}_{2}(167)_{\Delta}$-DHFR is arrested.

\subsubsection{An experimental setup to assay subunit integration into the active translocase}

In order to address the integration of subunits into the active translocase, an assay similar to the one used by Dekker et al. (1997) was established. The arrest of $b_{2}(167)_{\Delta}$-DHFR in only half of the import sites allowed subsequent import TIM23 substrates, not just TIM22 substrates. Additionally, isolation using a tagged TOM subunit allowed selective co-isolation of the active TIM23 complexes (i.e. those that were connected to TOM by the arrested $b_{2}(167)_{\Delta}$-DHFR). In contrast to the separation by BN-PAGE, this allowed analysis of subunits that are usually lost during the gel run (e.g. Tim50, Tim44, Pam16, Pam18). The specificity of the approach was demonstrated by the inability of Tom40, Tim23 and F1 $\beta$ to integrate into the supercomplex as well as the specific co-isolation of 
TOM, TIM23 and PAM subunits.

The channel-forming subunits are not exchanged because they are occupied by the precursor. This makes an exchange unfavorable, as the substrate would be exposed to the lipid bilayer without shielding. As the TOM complex forms two to three pores (Hill et al., 1998; Künkele et al., 1998b; Ahting et al., 2001), one can imagine a scenarios with only one pore (one Tom40 channel) being occupied. Therefore, exchange of the other Tom40 molecules should possible. This was not observed, which could be addressed to the stability of the Tom40-Tom22 core complex (Meisinger et al., 2001). Alternatively, the precursor might not be threaded through the $\beta$-barrel of Tom40, but through the space in the middle of several $\beta$-barrels (Harner et al., 2011).

The transient supercomplex that is generated during the import of a radiolabeled substrate is not sufficiently stable to co-isolate the TIM23 complex with Tom $22^{\text {His } 10}$. Hence specific recovery of a radiolabeled subunit with the TOM-TIM23 supercomplex is an indication that this subunit integrates into the active translocase.

It should be noted, that some TIM23 and PAM components were not accessible to this assay. The presence of Pam17 in the supercomplex is currently debated (van der Laan et al., 2005; Popov-Celeketić et al., 2008a). I could not co-isolate this subunit with the supercomplex and could therefore not detect integration into the active TIM23. Similarly, Mgr2 was only marginally detected in supercomplex isolations and hence not included. This was unexpected as Mgr2 is required for the generation of the TOM-TIM23 translocation intermediate (Gebert et al., 2012). Tim50 was excluded due to unspecific co-purification of the radiolabeled protein with Tom $22^{\mathrm{His} 10}$ independent of the $\mathrm{b}_{2}(167)_{\Delta^{-}}$ DHFR arrest (data not shown).

In contrast, $\left.{ }^{35} \mathrm{~S}\right]$ Tom 20 was found to integrate into the active translocase, when the isolations were performed using Tim23 ${ }^{\mathrm{PA}}$. While Tom22 was shown to be part of a highly stable subcomplex of TOM (Meisinger et al., 2001), Tom20 is know to be only peripherally associated (Ahting et al., 1999; van Wilpe et al., 1999). As the precursor is not actively engaged by the TOM complex when it is arrested I assume that only the $\beta$-barrel formed by Tom40 is required at this point. Hence, other TOM subunits might as well exchange with their free pool, however no physiological importance can be currently envisioned for this process. Of the tested TIM23 and PAM subunits Tim44, Pam18 and Tim21 were significantly enriched in the supercomplex. 


\subsubsection{Tim21, Tim44, and Pam18 oscillate in the active PAM complex}

Chacinska et al. (2010) showed that the TIM23 translocase does not exist as a single entity (containing TIM23 $3^{\mathrm{CORE}}$, Tim21 and PAM) nor as separate forms of TIM $23^{\mathrm{SORT}}$ and TIM $23^{\text {MOTOR }}$ but that it is in a dynamic equilibrium between the latter two.

Here I showed the interconversion of the sorting and the motor form by showing dynamic exchange of two motor subunits (Tim44 and Pam18) as well as Tim21 into the active translocase. A third motor subunit, Pam16, also showed weak exchange into the supercomplex, which might reflect the distance of the different subunits to the core of the translocase. Pam16 is bound to Tim44 (Kozany et al., 2004; Hutu et al., 2008; Schilke et al., 2012) and Pam18 to Tim17 and Pam16 (D'Silva et al., 2003; Truscott et al., 2003; Chacinska et al., 2005; D'Silva et al., 2008; Tamura et al., 2009). Hence Pam18, due to its more peripheral location, might be exchanged more readily. The same applies to Tim21 which is peripherally associated with TIM23 (van der Laan et al., 2006; Gebert et al., 2012). Even though the exchange of Pam16 was not further addressed in this work, it could be equally important for the transport process. The exchange of the regulatory motor subunits is strikingly similar to the cyclic recruitment of Hsp70 to TIM23. The implications of this are discussed below (see 3.3.6).

Most studies from different groups found that the Tim21 containing TIM23 ${ }^{\text {SORT }}$ does not contain stoichiometric amounts of PAM components (Chacinska et al., 2005; Mokranjac et al., 2005a; Popov-Celeketić et al., 2008a; Chacinska et al., 2010). Only one study could find small amounts of Pam16 and Pam18 in the same complex as Tim21 (Tamura et al., 2006). Additionally it was shown that supercomplexes arrested with $\mathrm{b}_{2}(167)_{\Delta^{-}}$ DHFR contains PAM subunits as well as Tim21, however not together in the same TIM23 (Mokranjac et al., 2005a; Chacinska et al., 2010). Therefore the data presented here can be interpreted in the following way: The TIM23 translocase with the arrested precursor can exist in the sorting or motor form. The Tim21-containing sorting form will not be able to efficiently retain the arrested precursor as that requires import motor activity (see above). Hence, the integration of Tim21 can not selectively occur only into the TIM23 $3^{\text {SORT }}$ form. Assuming that the subunits also leave the translocase at a similar rate as they are recruited, it can be envisioned that recruitment occurs to any TIM23 form. Hence the recruitment of motor subunits to the formerly Tim21-containing translocase 
would convert TIM23 $23^{\text {SORT }}$ into TIM $23^{\text {MOTOR }}$ and visa versa. The TIM $23^{\mathrm{CORE}}$ might be an intermediate in this process.

Several precursor proteins like Cox5a or cytochrome $\mathrm{b}_{2}$ are laterally sorted, but contain a long or folded domain after the presequence and before the TMD and are therefore motor dependent (Glick et al., 1993; Gärtner et al., 1995; Truscott et al., 2003). The sorting of precursor proteins was so far only demonstrated for the Tim21 containing TIM23 $3^{\text {SORT }}$ (van der Laan et al., 2007). However, as Tim21 is not essential and its deletion only results in an increased $\Delta \psi$ sensitivity during import of sorted precursor proteins, either TIM23 $3^{\mathrm{CORE}}$ or TIM23 $3^{\mathrm{MOTOR}}$ perform the lateral release in tim21 $\Delta$. In case the import motor occupies a putative lateral gate, one can speculate that the import motor has natural off-rate from the translocase enabling lateral release by TIM $23^{\mathrm{CORE}}$. The cycling of Tim21 described above might therefore facilitate the displacement of the import motor, probing of the precursor or lateral release when a sorting signal is detected by the translocase. Future studies are required to rigorously test this hypothesis.

\subsubsection{The structure and function of TIM23 and PAM in mutant mitochondria}

Previous studies frequently employed an overexpressed protein A tagged episomal copy of Tim23 to analyze the TIM23 composition (Frazier et al., 2004; Li et al., 2004; van der Laan et al., 2005). However, some of the results obtained from protein A tagged translocase subunits were debated (Popov-Celeketić et al., 2008a). Hence I used $\alpha$-Tim23 coimmunoprecipitations to analyze the presence of all known subunits at the TIM23 complex in different mutants. These immunoprecipitations specifically isolated all relevant subunits of the TIM23 and PAM complexes and only Hsp70 showed an ATP dependent dissociation from the translocase (Figure 31.A and B) (Schneider et al., 1994; Krimmer et al., 2000; Truscott et al., 2003; Frazier et al., 2004).

In the ssc1-3 mutant mitochondria no major changes in the subunit composition were detected (Figure 32.A), hence the drastic import defect after induction of the ts phenotype, is due to the defective nucleotide binding (Gambill et al., 1993; Voos et al., 1996).

In contrast in the temperature-sensitive mutant pam18-66 the amount of Pam18 at the translocase was reduced (Figure 32.B). These reduced Pam18 levels, would decrease the capacity to to stimulate Hsp70's ATPase activity at the exit site of the translocase, 
explaining the defects in import and inward driving activity. Similar results were obtained by Truscott et al. (2003) and Mokranjac et al. (2003b). They showed a normal TIM23 composition with respect to Tim50, Tim44, Tim23 and Tim17 in pam18-66 or mitochondria with down regulated Pam18.

In the pam16-3 mutant mitochondria a lack of Pam16 as well as Pam18 was observed (Figure 32.C). This is in line with previous studies using the same mutant or mitochondria with down regulated Pam16 (Frazier et al., 2004; Kozany et al., 2004). Additionally, Chacinska et al. (2005) showed increased levels of Tim21 using Tim23 ${ }^{\text {PA }}$ isolation. In the $\alpha$-Tim23 immunoprecipitations shown here, this effect was not seen. However, the Pam17 levels at the translocase were drastically increased, which could be interpreted as a compensatory effect as Pam17 was shown to be required for the assembly of the J-complex and its recruitment to TIM23 (van der Laan et al., 2005). Similar observations have also been made for other PAM16 mutants (Hutu et al., 2008; Schilke et al., 2012).

Matrix import efficiency of the temperature sensitive mutants pam16-3 and pam18-66 was surprisingly close to the respective WT or the control tim54-11 (Figure 35.A). This is in contrast to the lack of the subunits from the translocase, which would argue towards an import defect, as it has been reported (Truscott et al., 2003; Frazier et al., 2004). When mitochondria were challenged in the inward driving activity assay, the defects of the temperature sensitive alleles became more evident (Figure 36.C). Throughout this study temperature sensitive mutants showed milder defects compared to previous reports, this could be due to differences in handling (although the same parameters for incubation under restrictive conditions have been used).

In summary, as Pam16 recruits Pam18 to the translocase, mutations that affect Pam16 association with TIM23 also affect Pam18. Additionally mutations that interfere with the J-complex formation selectively reduce the level of Pam18 at the translocase.

Surprisingly, in pam17 $\Delta$ mitochondria the subunit composition of the translocase, as judged by the $\alpha$-Tim23 immunoprecipitations, was unaffected (Figure 34.A). Van der Laan et al. (2005) showed reduced Pam16 and Pam18 levels at the TIM23 complex, when Tim23 $3^{\mathrm{PA}}$ was isolated. Never the less, the absence of Pam17 led to a strong import phenotype in line with the previously reported results.

Apart from the reduced steady state level of Mgr2 and the consequently lower abundance at the translocase, TIM23 was unaffected in tim21 $\Delta$ mitochondria (Figure 34.B). This is in line with the peripheral association of Tim21 with the translocase, coupling it 
to the respiratory chain but not being responsible for TIM23 or PAM subunit recruitment or stabilization (Chacinska et al., 2005; van der Laan et al., 2006).

As expected, in mgr2 $\Delta$ mitochondria Tim21 levels at the TIM23 were found to be drastically reduced (Figure 34.C) (Gebert et al., 2012). Interestingly, the residual amounts of Tim21 recovered in the precipitation indicate that a fraction of Tim21 can still assemble to the translocase in the absence of Mgr2, something that can also be anticipated in Gebert et al. (2012) (Figure 2.B, lane 6 and Figure 2.C, lane 6). One explaination for this, could be the affinity of Tim $21^{\mathrm{IMS}}$ to Tim $50^{\mathrm{IMS}}$ and Tim $23^{\mathrm{IMS}}$, leading to an association of the subunits in the absence of Mgr2 (Lytovchenko et al., 2013). Due to a decreased inward driving activity (Figure 36.B and $\mathrm{C}$ ) the matrix import in mgr2 $\Delta$ is drastically reduced (Figure 35.A). While this is in line with previously reported data, the WT-like motorindependent lateral sorting mgr2 $\Delta$ was unexpected (Figure 35.B) (Gebert et al., 2012).

In conclusion, both pam17 $\Delta$ and mgr2 $\Delta$ are largely unaltered in their composition of the TIM23 complex, but display drastic import defects. This puzzling observation could be explained by the coupling defects between the TOM and TIM23 complex in mgr2 $\Delta$ (Gebert et al., 2012), or by an altered association kinetics or positioning of PAM subunits at the translocase exit site.

\subsubsection{Mgr2 is involved in the oscillation of Pam18}

As Tim44 and Pam18 efficiently integrated into the active translocase, they were good candidates to test whether their recruitment to TIM23 is altered in mgr2 $\Delta$ mitochondria. Pam18 assembled less efficiently, while Tim44 was not affected. Even though the translocase associated levels of Tim21 are reduced in mgr2 $\Delta$ this is not the reason for the reduced Pam18 assembly, as it assembled even better in tim21 $\Delta$. Hence Mgr2 modulates the kinetics of Pam18 assembly to the TIM23 complex. The reduced assembly still supports sufficient recruitment of Pam18 to the translocase at steady state. However, the reduced association kinetics render the import motor less efficient.

Just like Tim17, Mgr2 was shown to be in proximity of a translocating substrate (Gebert et al., 2012), hence one can not exclude a role of Mgr2 in the process of lateral sorting. Furthermore, it might be involved in the transition from TIM $23^{\text {SORT }}$ to TIM23 $3^{\text {MOTOR }}$ by regulating the Tim21 and motor assembly or it influences the correct positioning of Pam18 at the translocase. Detailed crosslinking assays investigating the 
positioning of the different PAM and TIM23 subunits could be used to investigate this hypothesis in the future (Hutu et al., 2008; Popov-Celeketić et al., 2011).

\subsubsection{Model of matrix translocation by the TIM23 complex}

Based on these results an updated model of protein translocation across the TIM23 complex can be proposed. While the precursor is inserted into the channel, Hsp70 undergoes multiple rounds of binding and release to drive translocation into the matrix by a ratchet mechanism. The regulatory and scaffolding PAM subunits Pam18 and Tim44 leave and integrate into the translocase during this process. It remains to be elucidated whether both subunits have the same oscillation frequency at the translocase as Hsp70.

One can imagine a mechanism where the Pam16-Pam18 complex is positioned at the translocase exit site and a conformational change of the J-complex is required to stimulate the ATPase activity of Hsp70 (Mokranjac et al., 2006). After activation of Hsp70, the interaction of Pam16-Pam18 is destabilized, resulting in a loss of Pam18. Tim21 could preferentially assemble to this complex, displacing the remaining PAM subunits and forming TIM23 $3^{\text {SORT }}$. Pam16 and Tim44 might also be lost from TIM23 directly after Pam18 after the Hsp70 stimulation. Tim21 would subsequently be recruited to TIM23 ${ }^{\text {CORE }}$. This process is most likely regulated by Mgr2 as it is the coupling partner for Tim21 and affects Pam18 assembly. The process could serve precursor probing in order to prevent complete matrix translocation of an inner membrane protein. The displacement of the import motor could be a prerequisite for lateral sorting in case the positioning of the PAM subunits at TIM23 blocks a potential lateral gate.

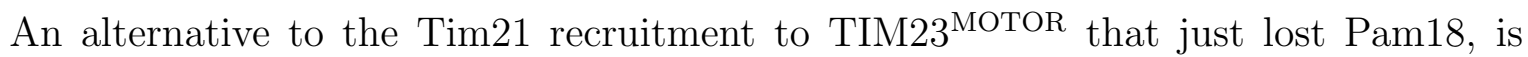
the assembly of a new Pam18 subunit. This would result in a "recharged" TIM23 2 MOTOR that can stimulate a new round of Hsp70 activity (Figure 41). The same would hold true for Tim44, except that its recruitment seems to be Mgr2 independent. As pam17 $\Delta$ mitochondria showed phenotypes similar to mgr2 $\Delta$ (all subunits at TIM23, strong import defect), similar investigations in this mutant could shed additional light on the regulation of the import motor.

Clearly, many of the steps of this proposed mechanism await to be tested by rigorous experiments. These would strongly benefit from a structure of the membrane embedded part of the presequence translocase. 


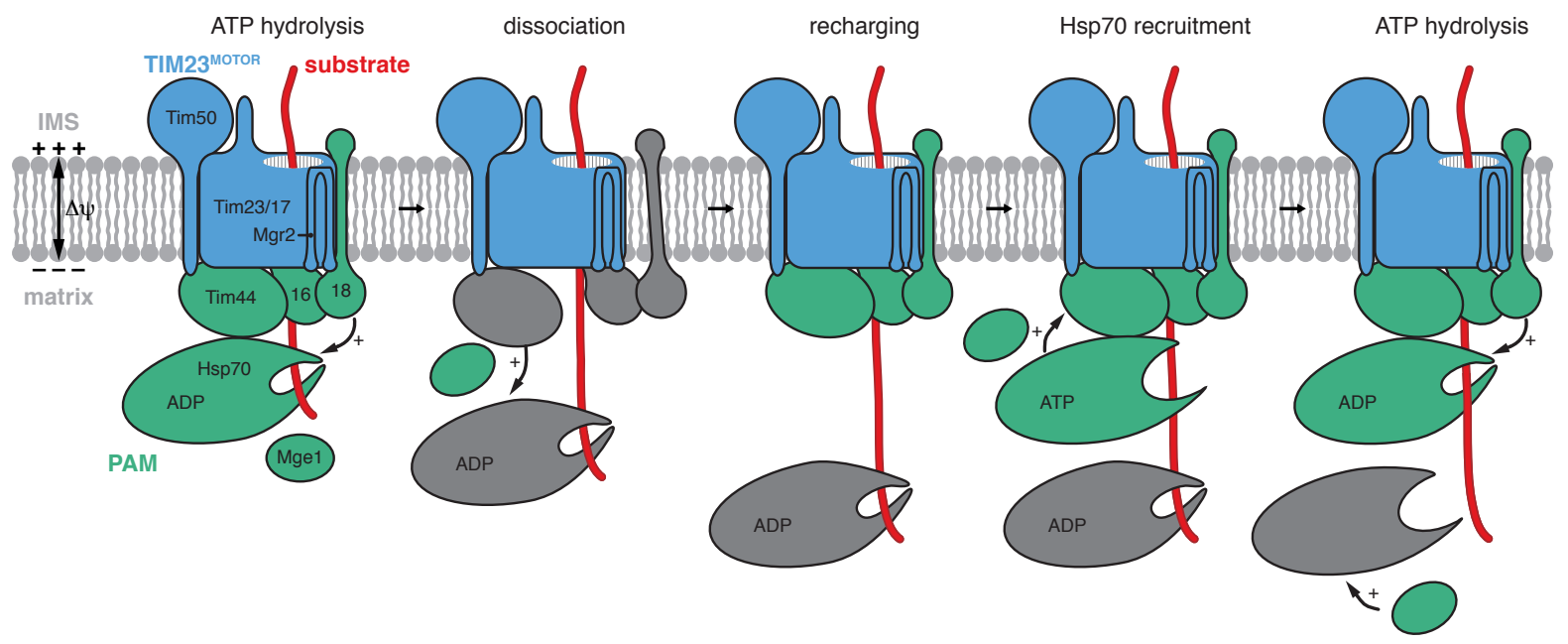

Fig. 41: Model of subunit oscillation during import motor function - During translocation of a polypeptide chain into the mitochondrial matrix ATP hydrolysis by Hsp70 is stimulated by Pam18. This leads to a destabilization of the regulatory PAM subunits (Pam16, Pam18 and Tim44) at the translocase and their subsequent dissociation from the exit site. For a new round of Hsp70 recruitment the translocase has to be recharged with these PAM subunits in order to support continuous Hsp70 stimulation and hence vectorial movement of the substrate. 


\section{Summary and Conclusion}

Within the eukaryotic cell mitochondria play an important role in metabolism and regulation of apoptosis. Due to their endosymbiotic origin and the transfer of most of its genetic information to the nucleus, the organelle largely relies on protein import for its biogenesis. Proteins carrying an N-terminal targeting signal (presequence) constitute the largest fraction among the imported proteins. Recognition of the presequence by the receptors Tom20 and Tom22 of the translocase of the outer membrane (TOM) initiates the transport process that will guide the proteins into the mitochondrial matrix or allow them to be laterally sorted into the inner membrane by the presequence translocase (TIM23).

In this thesis the mechanism of precursor recognition by the TIM23 complex as well as the regulation of the presequence translocase-associated import motor (PAM) are described. Using purified p-benzophenylalanine (BPA)-containing presequence peptides we identified Tim50 as the primary presequence receptor of the TIM23 complex. In its intermembrane space domain it contains a C-terminal presequence binding domain (PBD) as well as a second binding site in close proximity to a negatively charged groove. While both sites are sufficient to bind the targeting signal in vitro, the PBD is essential for cell viability due to its role in precursor recognition during protein transport across the inner membrane. We found that after initial presequence binding by Tim50, the targeting signal is then handed over to the channel-forming Tim23 via a trimeric intermediate. The role of the second binding site in Tim50 as well as the interplay of both binding sites with the receptor domain of Tim23 will be an attractive topic for future studies.

Once the precursor is transfered to the Tim23 channel, the membrane potential across the inner membrane drives transport of the positively charged presequence. However, full translocation into the mitochondrial matrix relies on the ATP driven import motor which consists of the mtHsp70, a tethering factor (Tim44), a J-protein (Pam18) as well as a regulatory J-like protein (Pam16). The activity of this motor is required to stabilize translocation intermediates that span the TOM and TIM23 complexes. Based on this, we developed an assay which revealed that Pam18 and Tim44 are able to integrate into 
the active TIM23 translocase. This integration is required for efficient protein import, as a TIM23 mutant (mgr2 $\Delta)$, defective in the recharging of the translocase with Pam18, shows drastic reductions in matrix import.

Due to the tight interaction of Pam18 with Pam16, conformational changes in Pam18 have long been speculated to be required for the stimulation of mtHsp70's ATPase activity. Does this lead to a subsequent destabilization of the interaction of Pam18 with the translocase? If so, one would expect that the exchange of regulatory subunits is coupled to the ATPase and recruitment cycle of mtHsp70. In the future it will be interesting to study potential conformational changes of the Pam18-Pam16 complex as well as possible synchronization of the regulatory PAM subunits with mtHsp70.

What would be the benefit of continuous recharging of the regulatory PAM subunits during import? This question might be answered by the present finding that the TIM23 subunit Tim21 also integrates into the active translocase. It has been previously shown that Tim21 association with TIM23 transforms the complex into a form that functions in lateral sorting of inner membrane proteins. Interestingly, Mgr2 which is required for efficient Pam18 assembly, is also important for Tim21 association. Hence, the labile association of regulatory PAM subunits and Tim21 could allow an Mgr2 mediated interconversion between the motor-associated and the sorting form of the TIM23 complex. Such a mechanism would be required to support biogenesis of proteins that contain a long segment between the presequence and the sorting signal and are therefore dependent on the import motor before they are released into the inner membrane. 


\section{Materials and Methods}

\section{$5.1 \quad$ Materials}

\subsubsection{Kit systems and reagents}

Standard chemicals obtained from AppliChem (Darmstadt, Germany), Merck (Darmstadt, Germany), Serva (Heidelberg, Germany), Sigma Aldrich (Taufkirchen, Germany), or Roth (Karlsruhe, Germany) were used in analytical grade. DNA primer were synthesized by Metabion (Martinsried, Germany). Commercial kit systems were used according to the manufacturers instructions and are listed in Table 5. Special chemicals and enzymes are listed in Table 6.

Tab. 5: Commercial kits used in this study and their supplier.

\begin{tabular}{ll}
\hline \hline Kit & Supplier \\
\hline Alkaline phosphatase, shrimp & Roche Applied Science \\
FastDigest restriction enzymes & Fermentas/ Thermo Scientific \\
GeneRuler DNA ladder 1 kb & Fermentas/ Thermo Scientific \\
High Pure PCR Template Preparation & Roche Applied Science (Mannheim, \\
Kit & Germany) \\
HMW calibration Kit & GE Healthcare \\
KOD Hot Start DNA Polymerase & Novagen/ Merck \\
mMessage Machine SP6 transcription & Ambion/ Life Technologies \\
Kit & \\
QuikChange Lightning Site-Directed & Agilent Technologies (Santa Clara CA, \\
Mutagenesis Kit & USA) \\
Rapid Ligation Kit & Thermo Scientific \\
TNT Flexi Translation & Promega (Mannheim, Germany) \\
TNT Quick Coupled Transcription/ & Promega \\
Translation SP6 & \\
\hline
\end{tabular}

Continued on next page 
Tab. 5 : Continued from previous page

\begin{tabular}{ll}
\hline Kit & Supplier \\
\hline Unstained SDS PAGE Protein marker & Serva (Heidelberg, Germany) \\
$6.5-200 \mathrm{kDa}$ & \\
Vivaspin (5000-MW cut-off) centricon & Sartorius stedim (Göttingen, \\
& Germany) \\
Wizard SV Gel and PCR Clean-Up & Promega \\
Wizard SV Mini-Prep & Promega \\
\hline \hline
\end{tabular}

Tab. 6: Reagents and their supplier used in this study.

\begin{tabular}{|c|c|}
\hline Reagent & Supplier \\
\hline$\left[{ }^{35} \mathrm{~S}\right]-\mathrm{L}-$ Methionine & $\begin{array}{l}\text { Hartmann Analytic (Braunschweig, } \\
\text { Germany) }\end{array}$ \\
\hline$\alpha-\mathrm{HA}$ & Sigma Aldrich \\
\hline$\alpha$-His6 & $\begin{array}{l}\text { Takara Clonetech (Sait-Germain-en-Laye, } \\
\text { France) }\end{array}$ \\
\hline Acrylamide, $4 \mathrm{x}$ crysatllized & Roth \\
\hline Agarose NEEO ultra quality & Roth \\
\hline Antimycin A & Sigma Aldrich \\
\hline ATP & Roche Applied Science \\
\hline BMOE & Thermo Scientific \\
\hline CNBr activated speharose $4 \mathrm{~B}$ & GE Healthcare \\
\hline Creatine phosphate & Roche Applied Science \\
\hline Creatine kinase & Roche Applied Science \\
\hline $\mathrm{CuSO}_{4} \cdot 5 \mathrm{H}_{2} \mathrm{O}$ & Merck \\
\hline DEPC & Roth \\
\hline DFDNB & Thermo Scientific \\
\hline Digitonin & Calbiochem/ Merck \\
\hline $\operatorname{DiSC}_{3}(5)$ & Invitrogen/ Life Technologies \\
\hline DMP & Sigma Aldrich \\
\hline
\end{tabular}

Continued on next page 
Tab. 6 : Continued from previous page

\begin{tabular}{|c|c|}
\hline Reagent & Supplier \\
\hline Dynabeads Protein G & Dynal/ Life Technologies \\
\hline Ethidiumbromide $0.07 \%$ & AppliChem \\
\hline Goat $\alpha$ Rabbit HRP & Dianova (Hamburg, Germany) \\
\hline Goat $\alpha$ Mouse HRP & Dianova \\
\hline Goat $\alpha$ Rabbit IR680 & LI-COR (Lincoln, NE, USA) \\
\hline Goat $\alpha$ Mouse IR680 & LI-COR \\
\hline Goat $\alpha$ Rabbit DyLight 488 & Dianova \\
\hline Goat $\alpha$ Mouse DyLight 488 & Dianova \\
\hline Hering Sperm DNA & Promega \\
\hline HisTrap HP $1 \mathrm{ml}, 5 \mathrm{ml}$ & GE Healthcare \\
\hline IgG from human serum & Sigma Aldrich \\
\hline IgG protein standard & BioRad (München, Germany) \\
\hline Imidazole & Merck \\
\hline $\mathrm{NADH}$ & Roche Applied Science \\
\hline NADPH & Roche Applied Science \\
\hline $\mathrm{Ni}^{2+}-\mathrm{NTA}$ agarose & Quiagen \\
\hline $\begin{array}{l}\text { N,N'-Methylene-bisacrylamide, } \\
2 \text { x crystallized }\end{array}$ & Serva \\
\hline Nourseothricin (ClonNAT) & $\begin{array}{l}\text { Werner BioAgents (Jena-Cospeda, } \\
\text { Germany) }\end{array}$ \\
\hline Oligomycin & Sigma Aldrich \\
\hline Poly-Prep column & BioRad \\
\hline Polypropylene coumn & Thermo Scientific \\
\hline Protein-A sepharose & GE Healthcare \\
\hline $\begin{array}{l}\text { Proteinase inhibitor cocktail } \\
\text { (EDTA-free) }\end{array}$ & Roche Applied Science \\
\hline Immobilon-P PVDF membrane & Millipore/ Merck \\
\hline Rabbit IgG & Sigma Aldrich \\
\hline Resource S 1ml, $6 \mathrm{ml}$ & GE Healthcare \\
\hline Rotiphorese ${ }^{\circledR}$ Gel 30 (37.5:1) & Roth \\
\hline
\end{tabular}

Continued on next page 
Tab. 6 : Continued from previous page

\begin{tabular}{ll}
\hline Reagent & Supplier \\
\hline RotiQuant & Roth \\
SnakeSkin dialysis membrane & Thermo Scientific \\
(7000-MW cut-off) & \\
Streptavidin agarose & Thermo Scientific \\
Streptavidin HRP & Dianova \\
Superdex 75 16/ 60 HiLoad & GE Healthcare \\
Valinomycin & Calbiochem/ Merck \\
X-ray films & Foma Bohemia (Hradec Králové, Czech \\
& Republic) \\
Zymolyase-20T & Seikagaku Biobusiness Corporation (Tokyo, \\
& Japan) \\
\hline \hline
\end{tabular}

\subsubsection{Microorganisms}

Saccharomyces cerevisiae YPH499 was used as the major wild-type strain, derivatives and other yeast strains are listed in Table 7. E. coli strains for cloning and protein expression are listed in Table 8. 


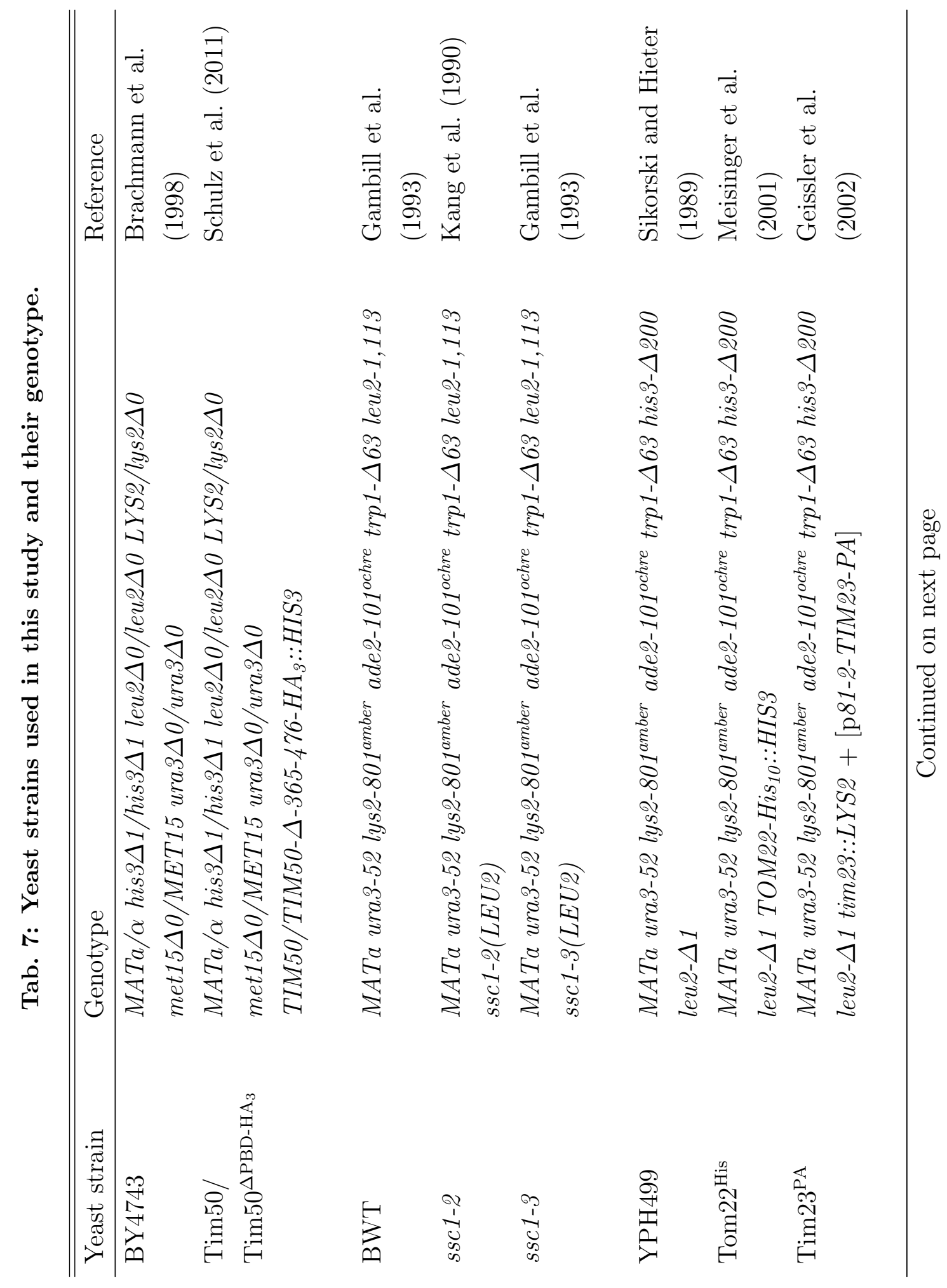




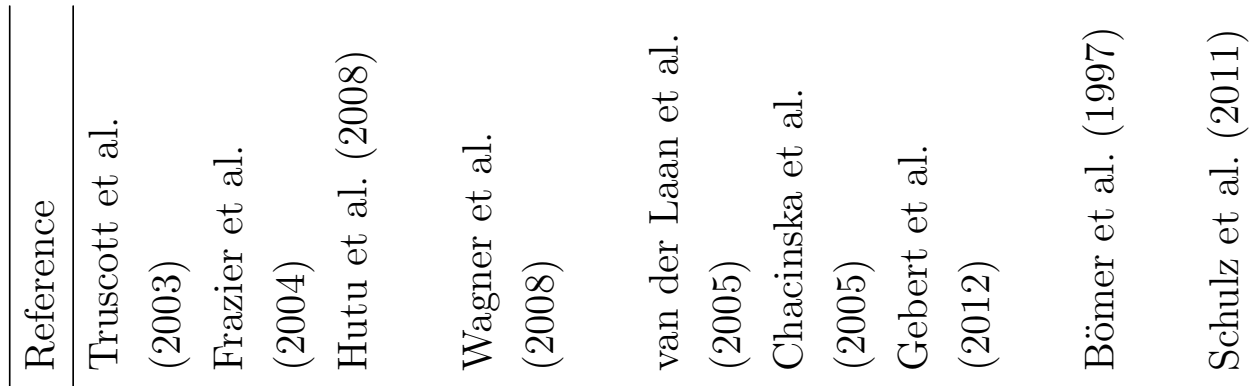

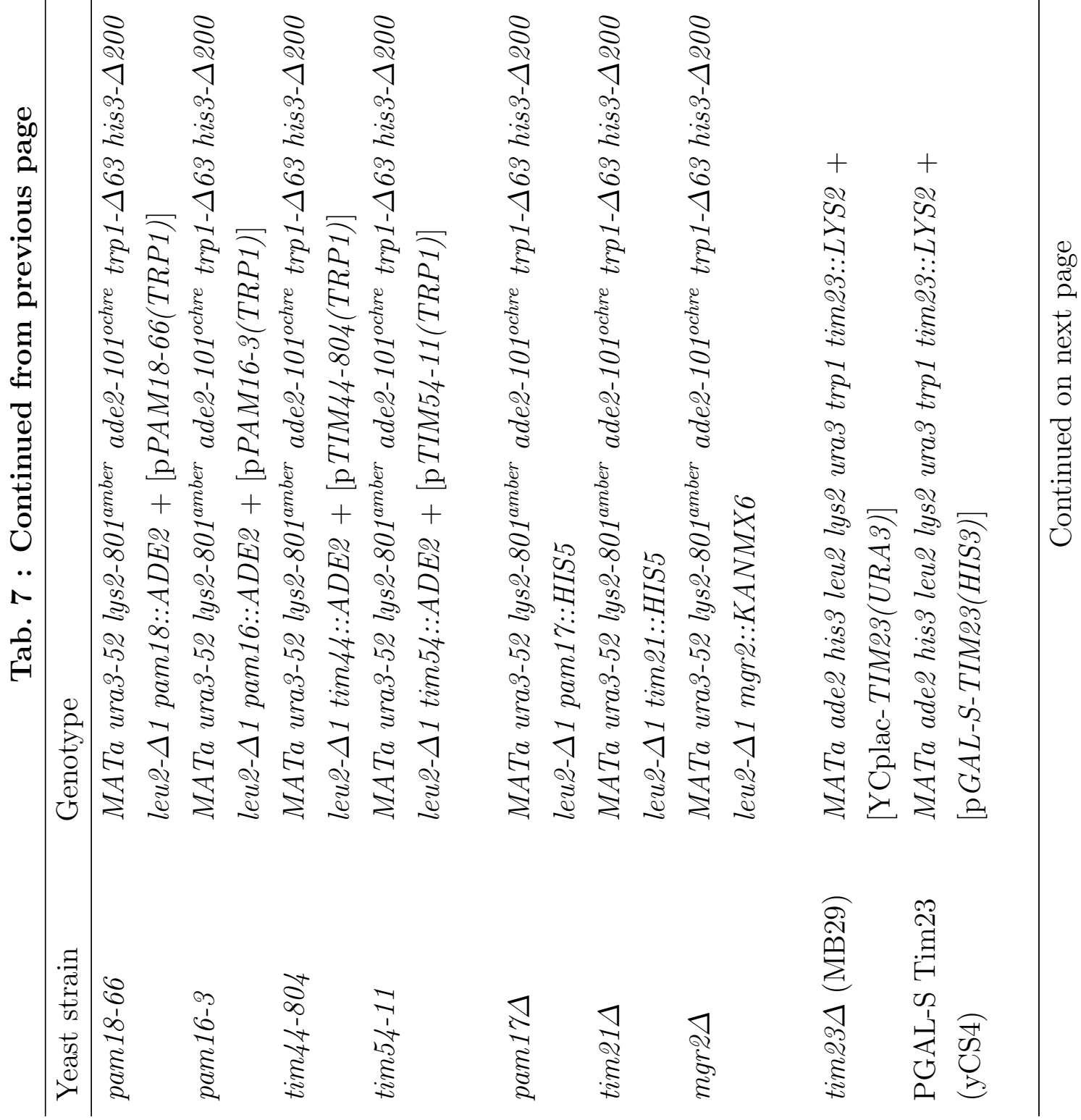




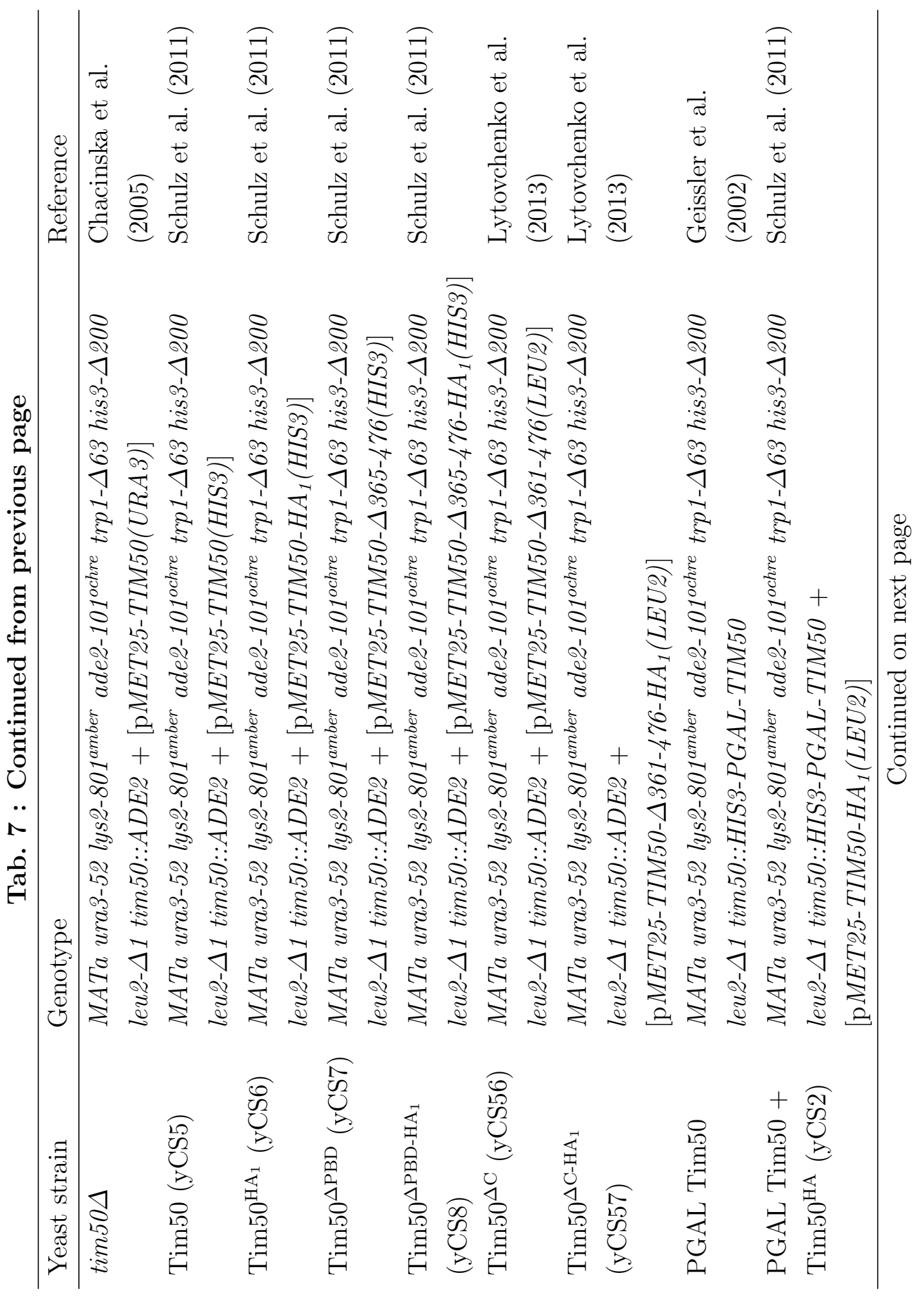




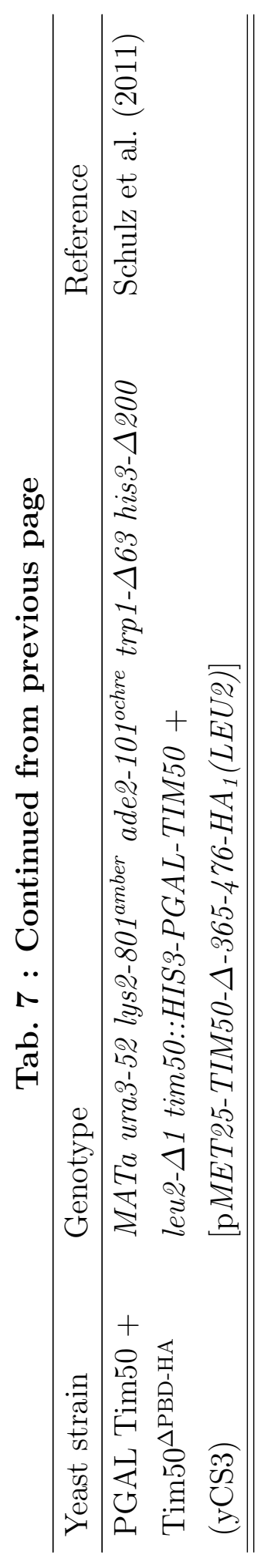


Tab. 8: $E$. coli strains used in this study and their genotype.

\begin{tabular}{|c|c|c|}
\hline E. coli strain & Genotype & Reference \\
\hline BMH7-18 & $\begin{array}{l}\Delta(\text { lac-pro } A B), \text { thi-1, supE, }\left[\mathrm{F}^{\prime} \text { traD36, }\right. \\
\left.\text { pro } A B^{+}, \text {lacI }^{q}, \text { lacZ } \Delta \mathrm{M} 15\right]\end{array}$ & $\begin{array}{l}\text { Dekker et al. } \\
(1997)\end{array}$ \\
\hline BL21 (DE3) & $\begin{array}{l}\text { fhuA2 [lon] ompT gal dcm hsdS } \lambda(\mathrm{DE} 3)\left(\mathrm{r}_{\mathrm{B}}^{-}\right. \\
\left.\mathrm{m}_{\mathrm{B}}^{-}\right) \mathrm{F}^{-}\end{array}$ & Stratagene \\
\hline XL1 Blue & $\begin{array}{l}\text { recA1 endA1 gyrA96 thi-1 hsdR17 supE44 } \\
\text { relA1 lac }\left[\mathrm{F}^{\prime} \text { proAB } \text { lacl }^{q} Z \Delta M 15 \operatorname{Tn} 10\right. \\
\left.\left(\text { Tet }^{\mathrm{r}}\right)\right]\end{array}$ & Stratagene \\
\hline
\end{tabular}

\subsubsection{Plasmids}

All plasmids used are listed in Table 9 and were propagated in E. coli XL1 Blue (Table 8). Cloning was performed as described in section 5.2.1.2 and 5.2.2.4. 


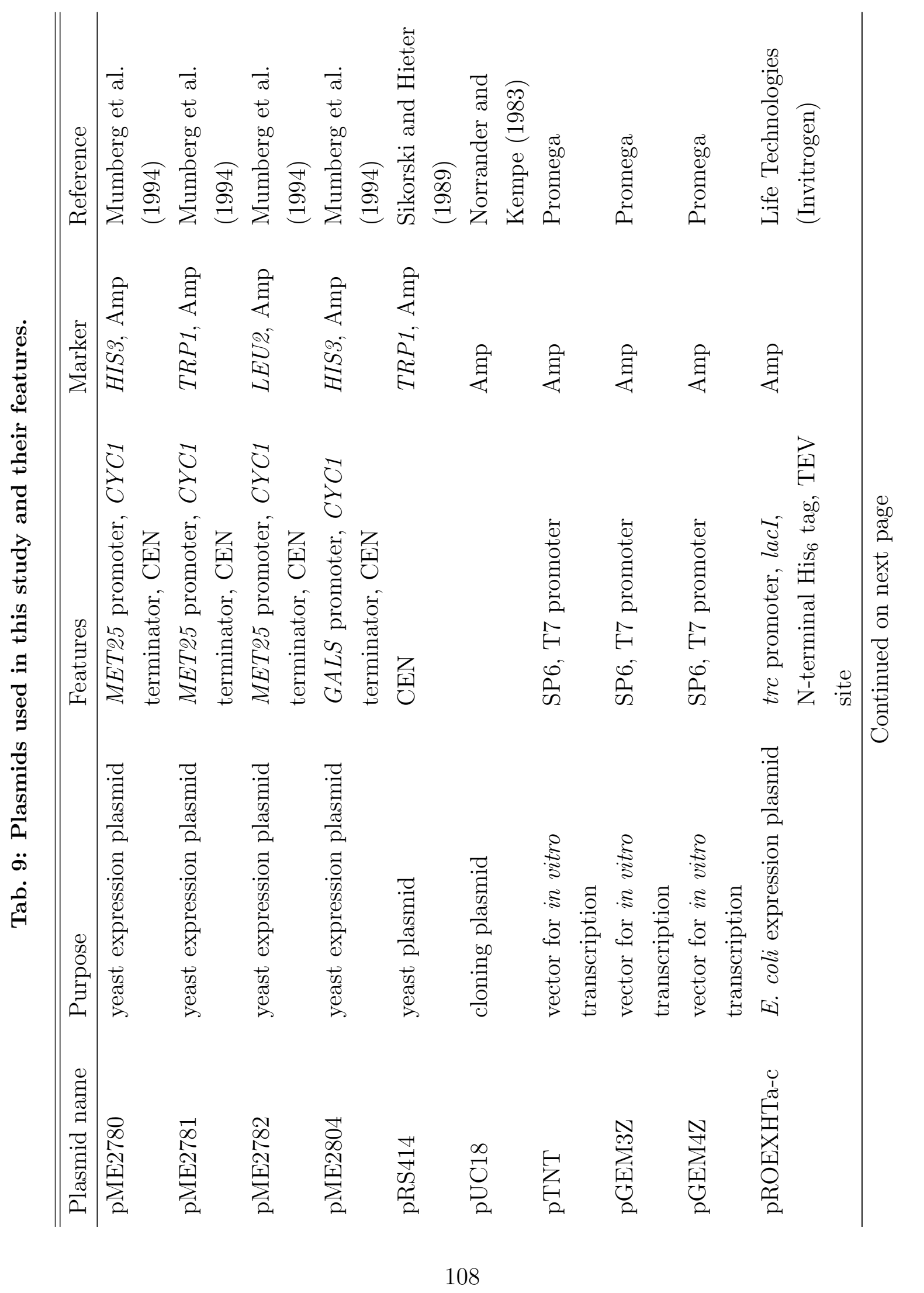




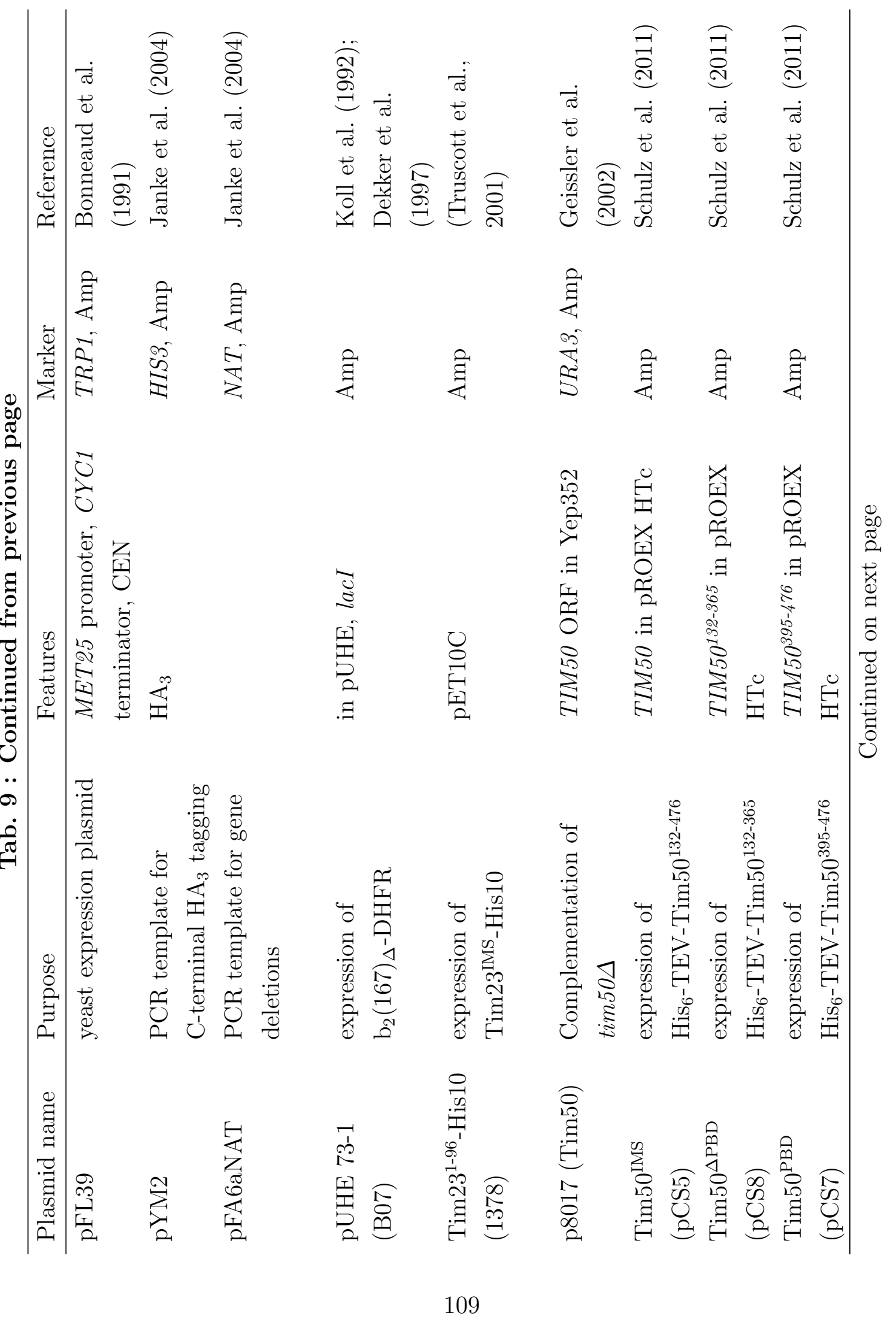




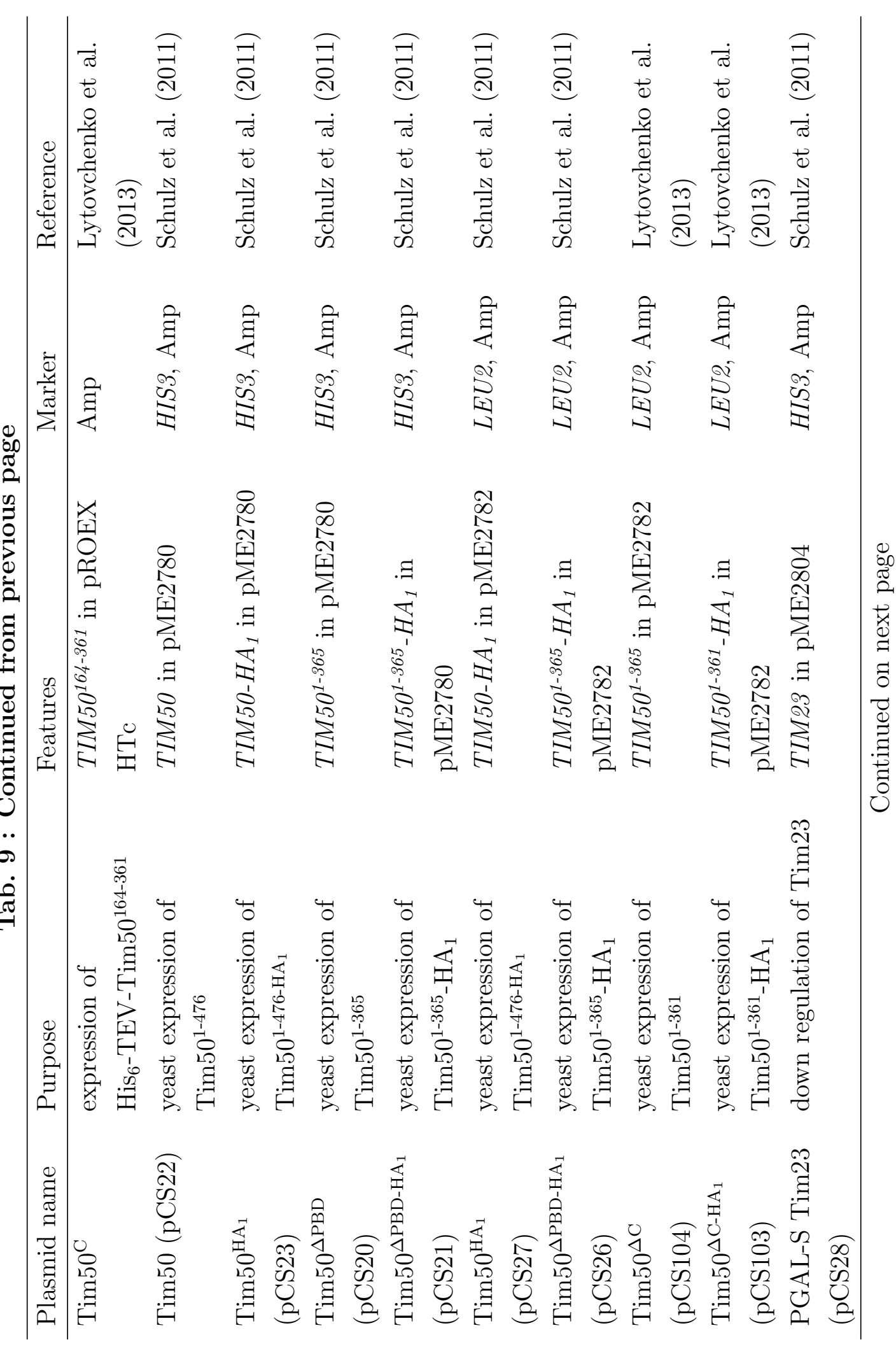




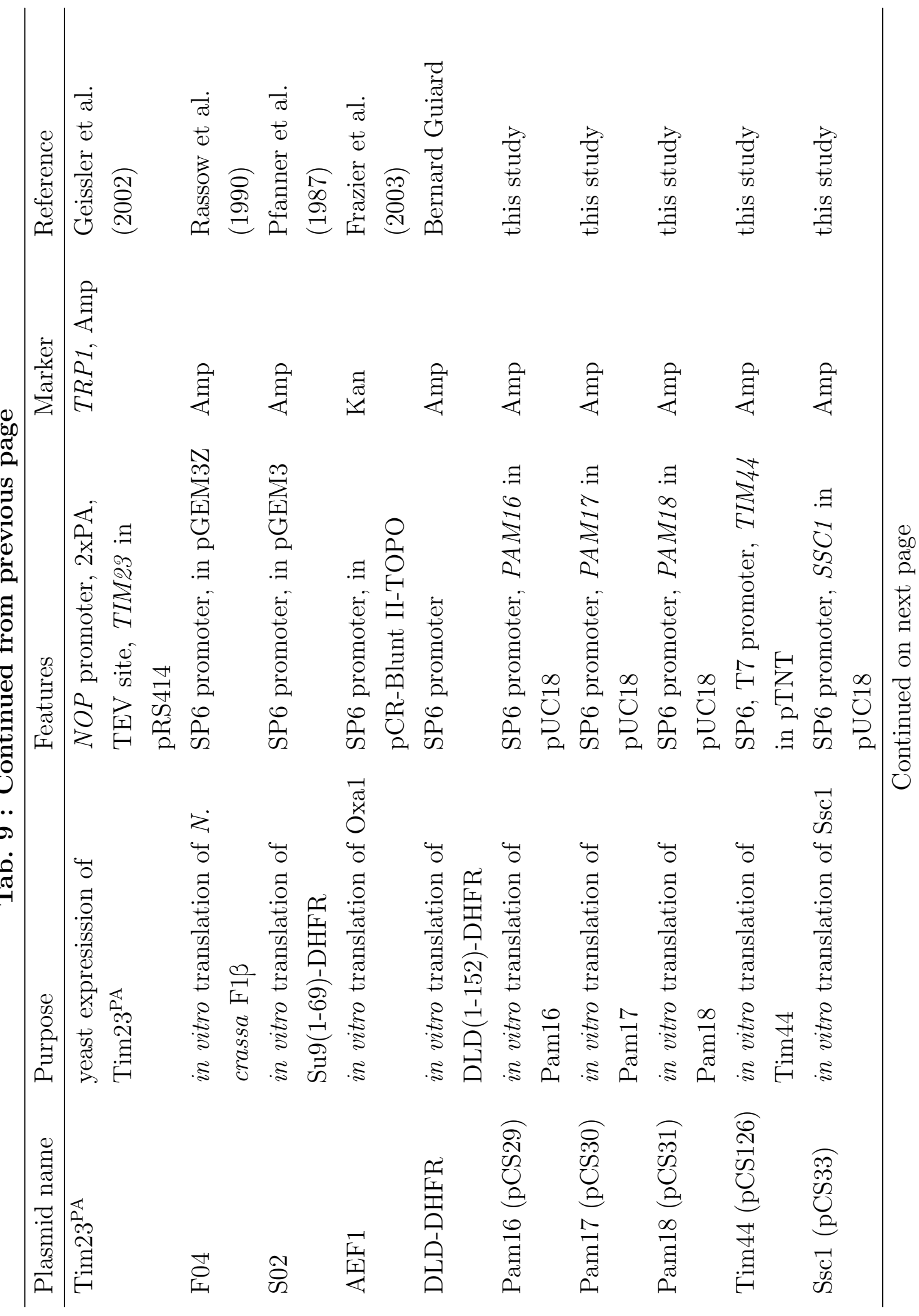




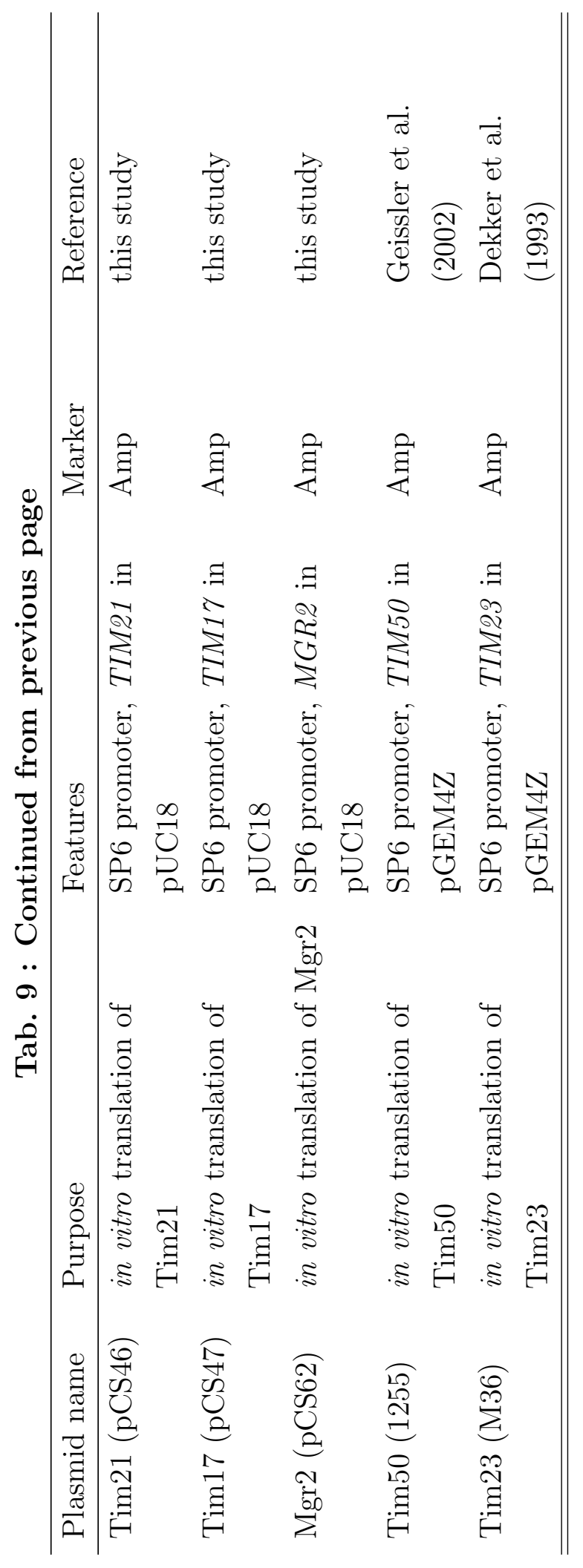




\subsubsection{Peptides}

Chemically synthesized peptides were dissolved in $10 \mathrm{mM}$ fresh acidic acid and stored in small aliquots at $-20^{\circ} \mathrm{C}$. They are listed in Table 10 .

Tab. 10: Peptides used in this study and their source.

\begin{tabular}{|c|c|c|c|}
\hline Peptide & Sequence ${ }^{a, b}$ & Source & Reference \\
\hline pALDH & MLRAALSTARRGPRLSRLLSAAA & $\begin{array}{l}\text { O. Jahn, } \\
\text { MPIem }^{c}\end{array}$ & $\begin{array}{l}\text { Hammen and } \\
\text { Weiner } \\
(1998)\end{array}$ \\
\hline $\begin{array}{l}\text { pALDH- } \\
\text { biotin }\end{array}$ & MLRAALSTARRGPRLSRLLSAAARXH $_{6}$ & $\begin{array}{l}\text { O. Jahn, } \\
\text { MPIem }^{c}\end{array}$ & $\begin{array}{l}\text { Schulz et al. } \\
(2011)\end{array}$ \\
\hline pALDH-s & MLRGKQPTKSLLPQRSPKLSAAA & $\begin{array}{l}\text { JPT, Berlin, } \\
\text { Germany }\end{array}$ & $\begin{array}{l}\text { Schulz et al. } \\
(2011)\end{array}$ \\
\hline pCox 4 & MLSLRQSIRFFKPATRTLCSSRYLL & $\begin{array}{l}\text { JPT, Berlin, } \\
\text { Germany }\end{array}$ & $\begin{array}{l}\text { Allison and } \\
\text { Schatz (1986) }\end{array}$ \\
\hline pCox4-Ser & MLSLRQSIRFFKPATRTLSSSRYLL & $\begin{array}{l}\text { JPT, Berlin, } \\
\text { Germany }\end{array}$ & $\begin{array}{l}\text { de la Cruz } \\
\text { et al. }(2010)\end{array}$ \\
\hline SynB2 & MLSRQQSQRQSRQQSQRQSRYLL & $\begin{array}{l}\text { JPT, Berlin, } \\
\text { Germany }\end{array}$ & $\begin{array}{l}\text { Allison and } \\
\text { Schatz (1986) }\end{array}$ \\
\hline $\mathrm{pL}_{19} \mathrm{~B}$ & MLRAALSTARRGPRLSRLBSAAARXH $_{6}$ & $\begin{array}{l}\text { O. Jahn, } \\
\text { MPIem }^{c}\end{array}$ & $\begin{array}{l}\text { Schulz et al. } \\
(2011)\end{array}$ \\
\hline $\mathrm{pS}_{16} \mathrm{~B}$ & MLRAALSTARRGPRLBRLLSAAARXH $_{6}$ & $\begin{array}{l}\text { O. Jahn, } \\
\text { MPIem }^{c}\end{array}$ & $\begin{array}{l}\text { Schulz et al. } \\
(2011)\end{array}$ \\
\hline
\end{tabular}

${ }^{a}$ B- p-Benzophenylalanine; X- biotinyllysin

${ }^{b}$ all peptides were synthesized as N-terminal amines and C-terminal amides

${ }^{c}$ Max-Planck-Institute of Experimental Medicine, Proteomics group, Göttingen, Germany

\subsubsection{Antibodies}

Primary polyclonal antibodies were produced by injecting antigen (synthetic peptide or purified proteins) into rabbits and the serum was diluted in TBS-T. Secondary goat antibodies directed against rabbit immunoglobulin were used as HRP (used 1:10.000) or fluorescent dye conjugates (used 1:5.000). When using $\alpha$-HA antibodies (Sigma), goat $\alpha$-mouse secondary antibodies were used (Table 6). 


\subsubsection{Instruments and software}

Instruments and software used in this study are listed in Table 11 and 12.

Tab. 11: Instruments used in this study

\begin{tabular}{ll}
\hline \hline Instrument & Manufacturer \\
\hline 5415 R (centrifuge) & Eppendorf \\
5424 (centrifuge) & Eppendorf \\
5804 R (centrifuge) & Eppendorf \\
F45-24-11 (centrifuge) & Eppendorf \\
Sorvall RC 6 Plus (centrifuge) & Thermo Scientific \\
Sorvall RC 12BP (centrifuge) & Thermo Scientific \\
JA-20 (rotor) & Beckman Coulter \\
Sorvall F14S-6x250Y (rotor) & Thermo Scientific \\
Sorvall SS-34 (rotor) & Thermo Scientific \\
Sorvall F10S-6x500Y (rotor) & Thermo Scientific \\
Sorvall H-12000 (rotor) & Thermo Scientific \\
TLA-55 (rotor) & Beckman Coulter \\
ÄKTA purifier (FPLC) & GE Healthcare \\
Curix 60 (developing machine) & AGFA \\
EmulsiFlex-C3 homogenizer & Avestin \\
GeneAmp PCR System 2700 (thermo cycler) & Applied Biosystems \\
F-7000 Fluorospectrometer & Hitachi \\
Fluorescence scanner FLA-9000 & Fuijfilm \\
LAS 3000 (LCD camera) & Fuijilim \\
Micro manipulator & Zeiss \\
Potter S (Dounce homogenisator) & Sartorius \\
Storm TM 820 PhosphorImager & GE Healthcare \\
Storage Phosphor screens & GE Healthcare \\
Thermomixer comfort & Eppendorf \\
UV Solo (UV documentation) & Biometra \\
\hline \hline & \\
\hline &
\end{tabular}


Tab. 12: Software used in this study

\begin{tabular}{ll}
\hline \hline Software & Producer \\
\hline DataGraph 3.1.1 & Visual Data Tools, Inc. \\
Geneious 5.3.6 & Biomatters, Auckland, New Zealand \\
Illustrator CS4 & Adobe Systems, San Jose, CA, USA \\
ImageQuant TL & GE Healthcare BioSciences AB, Uppsala, Sweden \\
Microsoft Office & Microsoft Corporation, Redmond, WA, USA \\
Papers 2 & Mekentosj, Aalsmeer, Netherlands \\
Photoshop CS4 & Adobe Systems, San Jose, CA, USA \\
PyMol & Schrödinger, Portland, OR, USA \\
\hline \hline
\end{tabular}

\subsection{Methods}

\subsubsection{Handling of biological material}

\subsubsection{E. coli growth}

E. coli were grown according to standard procedures using lysogeny broth (LB, $1 \%$ $\mathrm{NaCl}, 0.5 \%$ yeast extract, $1 \%$ tryptone) (Sambrook and Russell, 2001). Plates were supplemented with $15 \mathrm{~g} / \mathrm{L}$ agar. Antibiotic selection markers were used in the following concentrations: $0.1 \mathrm{~g} / \mathrm{L}$ ampicillin, $30 \mathrm{mg} / \mathrm{L}$ kanamycin. For protein expression detailed conditions are listed in Table $14 . \mathrm{b}_{2}(167)_{\Delta}$-DHFR was expressed as described before by Dekker et al. (1997), however the import-competence of the precursor was significantly increased when the main culture was inoculated to low OD (0.01). Cryo stocks of E. coli were generated by mixing $800 \mu \mathrm{L}$ of a culture in selective LB medium with $200 \mu \mathrm{L}$ sterile $80 \%$ glycerol in a cryo vial and freezing it at $-80^{\circ} \mathrm{C}$.

\subsubsection{E. coli transformation}

Preparation of chemically competent Escherichia coli was adapted from Hanahan (1983). Briefly, a $100 \mathrm{~mL}$ culture of an optical density ( $\mathrm{Abs}_{600 \mathrm{~nm}}$ ) of 0.6 was chilled on ice for $5 \mathrm{~min}$ and harvested by centrifugation for $5 \mathrm{~min}$ at $3300 \mathrm{rpm}$. The cells were resuspended in $40 \mathrm{~mL}$ ice cold buffer 1 (30 mM KAc, $100 \mathrm{mM} \mathrm{RbCl,} 10 \mathrm{mM} \mathrm{CaCl} 2,50 \mathrm{mM} \mathrm{MnCl} 2,15 \%$ glycerol, $\mathrm{pH} 5.8$ ) and chilled on ice for another 5 min. After centrifugation the cells were 
resuspended in $4 \mathrm{~mL}$ buffer $2\left(10 \mathrm{mM} \mathrm{MOPS}, 75 \mathrm{mM} \mathrm{CaCl}_{2}, 10 \mathrm{mM} \mathrm{RbCl}, 15 \%\right.$ glycerol, $\mathrm{pH}$ 6.5), aliquoted into $100 \mu \mathrm{L}$ and stored at $-80^{\circ} \mathrm{C}$. For transformation the cells were defrosted on ice, mixed with $200 \mathrm{ng}$ plasmid DNA or $10 \mu \mathrm{L}$ ligation mix, incubated for $15 \mathrm{~min}$ on ice and subsequently transferred to $42^{\circ} \mathrm{C}$ for $45 \mathrm{~s}$. Afterwards the cells were cooled down and shaken for one hour at $37^{\circ} \mathrm{C}$ with $1 \mathrm{~mL} \mathrm{LB}$ medium, before plating them onto appropriate selection plates.

\subsubsection{Yeast growth}

Unless noted otherwise, yeast were grown according to standard procedures at $30^{\circ} \mathrm{C}$ in YP medium (1\% yeast extract, $2 \%$ peptone) containing $2 \%$ glucose (YPD), $3 \%$ glycerol (YPG) or $3 \%$ lactate (pH 5.0 using KOH, YPL) shaking at $140 \mathrm{rpm}$ (Curran and Bugeja, 2006). Temperature sensitive strains were cultivated at $24^{\circ} \mathrm{C}$. Selective medium was prepared using $0.67 \%$ yeast nitrogen base without amino acids (YNB), $0.07 \%$ complete supplement mixture (CSM) lacking the appropriate metabolite and $2 \%$ sucrose or glucose. For multiple selection the essential components were added $(0.02 \mathrm{~g} / \mathrm{L}$ adenine hemisulfate, $0.02 \mathrm{~g} / \mathrm{L}$ L-histidine, $0.03 \mathrm{~g} / \mathrm{L}$ L-leucine, $0.03 \mathrm{~g} / \mathrm{L}$ L-lysine, $0.02 \mathrm{~g} / \mathrm{L} \mathrm{L-}$ tryptophan, $0.02 \mathrm{~g} / \mathrm{L}$ uracil) instead of CSM depending on the selection marker. Plates were supplemented with $25 \mathrm{~g} / \mathrm{L}$ agar. For the selection against URA marker containing plasmids, 5-fluoroorotic acid plates were used: $0.67 \%$ YNB, $0.077 \%$ CSM-ura, $2 \%$ glucose, $50 \mathrm{mg} / \mathrm{L}$ uracil, $650 \mathrm{mg} / \mathrm{L} 5$-FOA and $25 \mathrm{~g} / \mathrm{L}$ agar. In order to compare growth of different strains serial dilutions of cells were spotted on YPG or YPD plates and incubated at 24,30 and $37^{\circ} \mathrm{C}$ for $3-5 \mathrm{~d}$.

Yeast strains were preserved as cryo stocks by mixing material form an appropriate plate into cryo vials containing $1 \mathrm{~mL}$ of YPAD with $15 \%$ glycerol and storing them at $-80^{\circ} \mathrm{C}$.

\subsubsection{Transformation of yeast}

Competent yeast cells were prepared according to a modified protocol adapted from Gietz and Woods (2002). To this end, a $100 \mathrm{~mL}$ culture of 2x YPD supplemented with $80 \mathrm{mg} / \mathrm{L}$ adenine hemisulphate (YPAD) was grown to an optical density $\left(\mathrm{Abs}_{600}\right)$ of 2.0 at $30^{\circ} \mathrm{C}$. The culture was harvested by centrifugation for $5 \mathrm{~min}$ at $3000 \mathrm{xg}$ at $4{ }^{\circ} \mathrm{C}$ and washed once with sterile water and with $0.1 \mathrm{M}$ LiAc. Subsequently the cells were resuspended 
in $2 \mathrm{~mL} 0.1 \mathrm{M} \mathrm{LiAc}$ and centrifuged for $30 \mathrm{~s}$ at $12000 \mathrm{rpm}$. The pellet was resuspended in $2 \mathrm{~mL} 0.1 \mathrm{M} \mathrm{LiAc}$ and aliquoted into $100 \mu \mathrm{L}$. Cells were stored at $-80^{\circ} \mathrm{C}$. For the transformation, the cells were defrosted on ice and $12 \mu \mathrm{L}$ herring sperm DNA $(10 \mathrm{~g} / \mathrm{L})$ was added together with $300 \mathrm{ng}$ of plasmid DNA (see 5.2.2.2) or $2 \mu \mathrm{g}$ of purified PCR product (see 5.2.2.3). Following an incubation for $30 \mathrm{~min}$ at $30^{\circ} \mathrm{C}$ under brief agitation, $600 \mu \mathrm{L} \mathrm{LiAc}(0.1 \mathrm{M})$ and PEG $4000(40 \%$ (w/v)) were added and the cells were incubated for another $90 \mathrm{~min}$ at $30^{\circ} \mathrm{C}$ under shaking. Afterwards $68 \mu \mathrm{L}$ DMSO were added and the cells were transferred to $42^{\circ} \mathrm{C}$ for $15 \mathrm{~min}$. The cells were plated on the appropriate selection medium. When nourseothricin (NAT) was used as a selection marker the cells were pelleted and resuspended in $2 \mathrm{~mL} 2 \mathrm{x}$ YPAD and incubated for $3 \mathrm{~h}$ at $30^{\circ} \mathrm{C}$ before plating. For temperature sensitive strains all incubations were carried out at $24^{\circ} \mathrm{C}$.

\subsubsection{Sporulation and dissection of tetrads}

Diploid BY4743 were sporulated according to an protocol adapted from Treco and Winston (2001). Briefly, a stationary YPD culture was washed in sporulation medium $(2 \%$ KAc, $0.2 \%$ yeast extract, $0.1 \%$ glucose, $0.2 \%$ leucine, $0.04 \%$ histidine, lysine and uracil) and further grown in the same medium for $5 \mathrm{~d}$ at $24^{\circ} \mathrm{C}$. A fraction of the culture was washed with $\mathrm{dH}_{2} \mathrm{O}$ and treated with zymolyase $(0.5 \mathrm{mg} / \mathrm{mL})$ for $20 \mathrm{~min}$ at $30^{\circ} \mathrm{C}$. Tetrads were dissected by running $6 \mu \mathrm{L}$ of the cell suspension over a YPD plate and using a micromanipulator (Zeiss) to separate them. Subsequently, the cells were grown at $30^{\circ} \mathrm{C}$.

\subsubsection{Whole cell lysate of yeast}

In order to analyze correct expression of proteins whole cell lysates were prepared (adapted from Cox et al. (1997)) and analyzed by SDS-PAGE and western blotting. To this end, yeast cells were cultured in appropriate medium and $5 \times 10^{7}$ (approximately $5 \mathrm{OD}_{600}$ ) cells were harvested by centrifugation for $5 \mathrm{~min}$ at $2500 \mathrm{xg}$ at $4^{\circ} \mathrm{C}$. Subsequently the cells were washed with $1 \mathrm{~mL}$ of $\mathrm{dH}_{2} \mathrm{O}$, centrifuged again and resuspended in $315 \mu \mathrm{L} 4.7 \mathrm{mM} \mathrm{KPi}$ $\mathrm{pH}$ 7.4. TCA was added to $15 \%$ and the samples were incubated for $30 \mathrm{~min}$ at $-80^{\circ} \mathrm{C}$. Afterwards the sample was defrosted and centrifuged for $10 \mathrm{~min}$ at $16100 \mathrm{xg}, 4^{\circ} \mathrm{C}$. The supernatant was discarded and the pellet was washed with $80 \%$ ice cold acetone. The precipitated material was resuspended in $25 \mu \mathrm{L} 1 \% \mathrm{SDS} / 0.1 \mathrm{M} \mathrm{NaOH}$ and SDS sample buffer was added to $1 \mathrm{x}$. 


\subsubsection{Preparation of S. cerevisiae mitochondria}

A crude mitochondrial fraction was isolated from yeast according to Stojanovski et al. (2007). To this end, cells were grown $\sim 2.0 \mathrm{OD}_{600}$ and harvested by centrifugation $(7000 \mathrm{xg}$ for $15 \mathrm{~min}$ at $18^{\circ} \mathrm{C}$ ). The wet weight of the cells was determined and the pellet was resuspended at $2 \mathrm{~mL} / \mathrm{g}$ in DTT buffer (100 mM Tris $/ \mathrm{H}_{2} \mathrm{SO}_{4} \mathrm{pH} 9.4$ and $10 \mathrm{mM}$ DTT). After shaking the suspension for $1 \mathrm{~h}$ at $30^{\circ} \mathrm{C}$ with $90 \mathrm{rpm}$, the cells were pelleted $(7000 \mathrm{xg}$ for $8 \mathrm{~min}$ at $18^{\circ} \mathrm{C}$ ), washed with $200 \mathrm{~mL}$ zymolyase buffer $(20 \mathrm{mM} \mathrm{KPi} \mathrm{pH} \mathrm{7.4,} 1.2 \mathrm{M}$ sorbitol) and subsequently resuspended at $7 \mathrm{~mL} / \mathrm{g}$ in zymolyase buffer containing $4 \mathrm{mg} / \mathrm{g}$ zymolyase.

Following an $1 \mathrm{~h}$ incubation at $30^{\circ} \mathrm{C}$ with $90 \mathrm{rpm}$ the rate of spheroblast generation

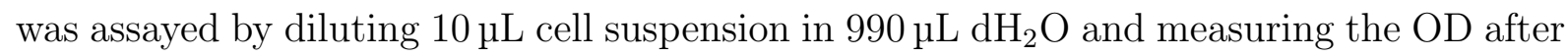
2 min. Spheroblasts were generated successfully when the OD after zymolyase treatment was below $10 \%$ of the sample without treatment. Subsequently the cells were collected by centrifugation for $10 \mathrm{~min}$ at $5000 \mathrm{xg}$ at $18^{\circ} \mathrm{C}$ and washed once more with $200 \mathrm{~mL}$ zymolyase buffer. Afterwards the cells were resuspended at $7 \mathrm{~mL} / \mathrm{g}$ in cold homogenization buffer (10 mM Tris/HCl pH 7.4, $600 \mathrm{mM}$ sorbitol, $1 \mathrm{mM}$ EDTA, $2 \mathrm{~g} / \mathrm{L}$ fatty-acid free BSA and $1 \mathrm{mM}$ PMSF). The suspension was homogenized using a $60 \mathrm{~mL}$ Dounce homogenizer (potter) at $800 \mathrm{rpm}$ for 20 strokes on ice. The homogenate was centrifuged for $5 \mathrm{~min}$ at $5000 \mathrm{xg}$ at $4{ }^{\circ} \mathrm{C}$. The resulting supernatant was centrifuged $10 \mathrm{~min}$ at $7000 \mathrm{xg}$ at $4{ }^{\circ} \mathrm{C}$. The crude mitochondrial fraction was pelleted by centrifugation for $15 \mathrm{~min}$ at $17000 \mathrm{xg}$. The resulting pellet was resuspended in $5 \mathrm{~mL}$ SEM buffer (250 mM sucrose, $20 \mathrm{mM}$ MOPS/ $\mathrm{KOH}$ pH 7.2 and $1 \mathrm{mM}$ EDTA) containing $1 \mathrm{mM}$ fresh PMSF. Pooled pellets were centrifuged again and resuspended in an appropriate volume of SEM buffer. Protein concentration was determined using $10 \%$ RotiQuant (Roth) and an IgG standard (BioRad) at 7.5, 15, 30 and $60 \mu \mathrm{g} / \mathrm{mL}$ and a 1:10 dilution of the mitochondria in $\mathrm{dH}_{2} \mathrm{O}$. Absorption at $595 \mathrm{~nm}$ was measured and mitochondria were adjusted to $10 \mu \mathrm{g} / \mathrm{\mu L}$ using SEM buffer. Aliquots of appropriate volume were flash frozen in liquid nitrogen and stored at $-80^{\circ} \mathrm{C}$.

In order to prepare mitochondria from mgre $\Delta$ cells they were grown to $\sim 2.0 \mathrm{OD}_{600}$ and subsequently shifted to $39^{\circ} \mathrm{C}$ for $24 \mathrm{~h}$ (Gebert et al., 2012). Afterwards the preparation was carried out as described above. 


\subsubsection{Molecular biology}

\subsubsection{Isolation of yeast genomic DNA}

YPH499 was cultured at $30^{\circ} \mathrm{C}$ in YPD overnight. $10^{8}$ cells (approximately 10 OD) were used to isolate genomic DNA using the "High Pure PCR Template Kit" (Roche) according to the manufacturers instructions. Briefly the cells were collected by centrifugation for $5 \mathrm{~min}$ at $3000 \mathrm{xg}$. The pellet was resuspended in $200 \mu \mathrm{L}$ PBS $(137 \mathrm{mM} \mathrm{NaCl}, 2.7 \mathrm{mM}$ $\mathrm{KCl}, 10 \mathrm{mM} \mathrm{Na} \mathrm{HPO}_{4}, 1.8 \mathrm{mM} \mathrm{KH_{2 }} \mathrm{PO}_{4}$ ). Zymolyase was added to $0.25 \mathrm{mg} / \mathrm{mL}$ and the suspension was incubated for $30 \mathrm{~min}$ at $37^{\circ} \mathrm{C}$. Subsequently $200 \mu \mathrm{L}$ binding buffer and $40 \mu \mathrm{L} 1 \mathrm{mg} / \mathrm{mL}$ PK were added, the sample was mixed and incubated for $10 \mathrm{~min}$ at $70{ }^{\circ} \mathrm{C}$. After addition of $100 \mu \mathrm{L}$ isopropanol the sample was applied to the filter tube and centrifuged for $1 \mathrm{~min}$ at $8000 \mathrm{xg}$. The filter tube was washed with $500 \mathrm{\mu L}$ inhibitor removal buffer and $500 \mu \mathrm{L}$ wash buffer. The DNA was eluted using $200 \mu \mathrm{L}$ prewarmed elution buffer. Genomic DNA was stored at $-20^{\circ} \mathrm{C}$.

\subsubsection{Isolation of plasmid DNA from $E$. coli}

Plasmid DNA was isolated using the "Wizard Plus SV Minipreps DNA Purification System" (Promega) according to the manufacturers instructions. Briefly, 2-5 mL of an E. coli culture in LB medium were harvested by centrifugation for 3 min at $12000 \mathrm{xg}$. The pellet was resuspended in $250 \mu \mathrm{L}$ resuspension buffer, $250 \mu \mathrm{L}$ lysis buffer were added along with $10 \mu \mathrm{L}$ alkaline protease. The sample was inverted 6 times and incubated at room temperature for $5 \mathrm{~min}$. Subsequently $350 \mu \mathrm{L}$ neutralization buffer were added and the samples were centrifuged for $10 \mathrm{~min}$ at $20200 \mathrm{xg}$. The supernatant was loaded on a filter tube and centrifuged for $1 \mathrm{~min}, 20200 \mathrm{xg}$. The filter was washed with 750 and $250 \mu \mathrm{L}$ washing buffer, and was spun dry afterwards. The DNA was eluted by incubation of the filter with

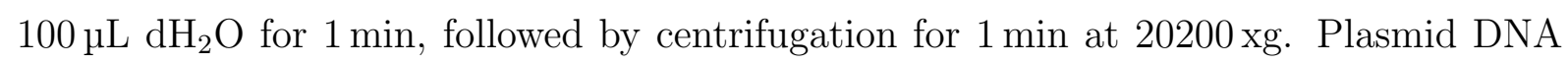
was stored at $-20^{\circ} \mathrm{C}$

\subsubsection{PCR}

DNA segments were amplified from yeast genomic DNA or plasmid DNA by the polymerase chain reaction using KOD polymerase (Novagen). To this end, reactions containing $0.4 \mu \mathrm{M}$ forward and reverse primer, 10-100 ng template DNA, 1X KOD buffer, $1.5 \mathrm{mM}$ 
$\mathrm{MgSO}_{4}, 0.2 \mathrm{mM}$ of each dNTPs and $1 \mathrm{U}$ KOD Hot Start DNA polymerase were prepared. Cycling conditions were: $2 \mathrm{~min}$ at $95^{\circ} \mathrm{C}$ for polymerase activation, $20 \mathrm{~s}$ at $95^{\circ} \mathrm{C}, 10 \mathrm{~s}$ at $48-55^{\circ} \mathrm{C}$ (depending on the primer combination used) and 10 to $25 \mathrm{~s} / \mathrm{kb}$ (depending on the length of the product) for cycling (35 cycles). PCR products were analyzed by agarose gel electrophoresis (1\% low melting agarose (Roth) in 1x TAE buffer (40 mM Tris, $20 \mathrm{mM}$ acetic acid and $1 \mathrm{mM}$ EDTA) in horizontal electrophoresis cell (BioRad) at $110 \mathrm{~V}$ for $30 \mathrm{~min}$. PCR products were purified using the Wizard SV Gel and PCR CleanUp System (Promega). Briefly, the DNA fragment was mixed with an equal volume of binding buffer (however resulting in at least $200 \mu \mathrm{L}$ ), loaded on a filter tube, incubated $1 \mathrm{~min}$ at room temperature and centrifuged for $1 \mathrm{~min}$ at $20200 \mathrm{xg}$. The filter was washed with 700 and $500 \mu \mathrm{L}$ washing buffer, and subsequently the filter was spun dry. Elution

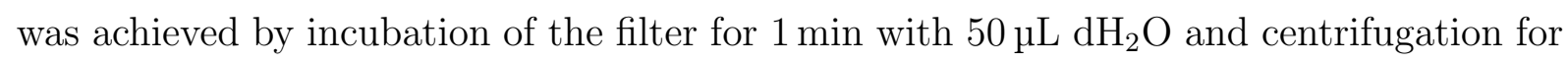
$1 \mathrm{~min}$ at $20200 \mathrm{xg}$. Purified DNA fragments were stored at $-20^{\circ} \mathrm{C}$.

\subsubsection{Cloning}

Clonging was carried out according to standard procedures (Sambrook and Russell, 2001). In order to insert purified PCR products into plasmids, both were first digested with the appropriate restriction enzymes (FastDigest (Fermentas/ Thermo Scientific)). To this end, $30 \mu \mathrm{L}$ reactions were prepared containing $1 \mathrm{x}$ FastDigest buffer, $1 \mu \mathrm{L}$ of each restriction enzyme and 800-1000 ng DNA. Restriction was carried out for $30 \mathrm{~min}$ at $37^{\circ} \mathrm{C}$ with slight agitation (400 rpm). Optionally, the opened plasmid was dephosphorylated using $1 \mu \mathrm{L}$ alkaline phosphatase and addition of the phosphatase buffer to a final concentration of $1 \mathrm{x}$ to the reaction. Fragments were purified (see 5.2.2.3) and mixed: $5 \mu \mathrm{L}$ of plasmid, $10 \mu \mathrm{L}$ of insert and $4 \mu \mathrm{L}$ DNA ligation buffer and $1 \mu \mathrm{L}$ T4 DNA ligase (Rapid DNA Ligation Kit, Thermo Scientific). After incubation for $30 \mathrm{~min}$ at $21^{\circ} \mathrm{C}, 10 \mu \mathrm{L}$ of the ligation reaction were used to transform $50 \mu \mathrm{L}$ competed XL1 Blue E. coli (see 5.2.1.2). Constructs were analyzed by analytical restriction digest and sequencing (GATC Biotech AG, Konstanz, Germany).

\subsubsection{In vitro mutagenesis}

Mutations were introduced into purified plasmids using the "QuikChange Site-Directed Mutagenesis Kit" (Agilent Technologies). Partially overlapping primers containing the 
mutations were designed according to Zheng (2004). Reactions $(50 \mu \mathrm{L})$ contained $50 \mathrm{ng}$ plasmid DNA, $0.2 \mu \mathrm{M}$ forward and reverse primer, $1 \mu \mathrm{L}$ dNTP mix, $1 \mathrm{x}$ buffer and $2.5 \mathrm{U}$ PfuTurbo DNA polymerase. After a initial denaturation for $30 \mathrm{~s}$ at $95^{\circ} \mathrm{C}$, cycling conditions were $30 \mathrm{~s}$ at $95^{\circ} \mathrm{C}, 1 \mathrm{~min}$ at $48-55^{\circ} \mathrm{C}, 1 \mathrm{~min} / \mathrm{kb}$ at $68^{\circ} \mathrm{C}$ for 20 times. Subsequently the reaction was cooled down on ice for $2 \mathrm{~min}, 10 \mathrm{U}$ DpnI were added in order to digest the parental DNA for $1 \mathrm{~h}$ at $37^{\circ} \mathrm{C}$. $5 \mu \mathrm{L}$ of the reaction were used to transform $50 \mu \mathrm{L}$ competent XL1 Blue E. coli (see 5.2.1.2). Success of the mutation was determined by analytical restriction digestion and/ or sequencing.

\subsubsection{In vitro transcription}

In order to generate capped mRNA's in vitro, PCR products containing a SP6 promoter in front of the ORF were transcribed using the mMESSAGE mMACHINE SP6 kit (Life Technologies). To this end, $20 \mu \mathrm{L}$ reactions were prepared, containing: 1x NTP/ CAP, $1 \mathrm{x}$ reaction buffer, $1 \mu \mathrm{g}$ PCR product and $2 \mu \mathrm{L}$ enzyme mix. Transcription was performed for $90 \mathrm{~min}$ at $37^{\circ} \mathrm{C}$, afterwards $2 \mathrm{U}$ TURBO DNaseI were added, followed by another $15 \mathrm{~min}$ at $37^{\circ} \mathrm{C}$. In order to recover the mRNA, $30 \mu \mathrm{L}$ nuclease-free water and $30 \mu \mathrm{L}$ $\mathrm{LiCl}$ precipitation solution (7.5 M lithium chloride and $50 \mathrm{mM}$ EDTA) were added, the sample was mixed and frozen at $-20^{\circ} \mathrm{C}$ for at least $30 \mathrm{~min}$. Subsequently, the sample was centrifuged for $15 \mathrm{~min}$ at $16100 \mathrm{xg}$ and $4^{\circ} \mathrm{C}$. The RNA pellet was washed with $1 \mathrm{~mL} 70 \%$ ethanol, centrifuged and dried. Finally the RNA was resuspended in $50 \mu \mathrm{L}$ DEPC water. mRNA was stored at $-80^{\circ} \mathrm{C}$.

\subsubsection{In vitro translation}

Translation was performed from plasmid templates (TNT ${ }^{\circledR}$ Quick Coupled Transcription Translation kit (Promega)) or mRNA (Flexi ${ }^{\circledR}$ Rabbit Reticulocyte Lysate System (Promega)). For the coupled reaction $40 \mu \mathrm{L}$ TNT ${ }^{\circledR}$ Quick Master Mix were mixed with $1 \mu \mathrm{g}$ plasmid DNA and $50 \mu \mathrm{Ci}{ }^{35} \mathrm{~S}-$ Met. For the translation from mRNA $33 \mu \mathrm{L}$ Flexi ${ }^{\circledR}$ Rabbit Reticulocyte Lysate, $1 \mu \mathrm{L} 1 \mathrm{mM}$ amino acid mix without methionine, $1.5 \mu \mathrm{g} \mathrm{mRNA}$ and $50 \mu \mathrm{Ci}{ }^{35} \mathrm{~S}-\mathrm{Met}$. Additionally $\mathrm{KCl}$ and $\mathrm{MgAc}$ where added to $70-120 \mathrm{mM}$ and $0-2 \mathrm{mM}$, depending on the precursor synthesized (see Table 13). 


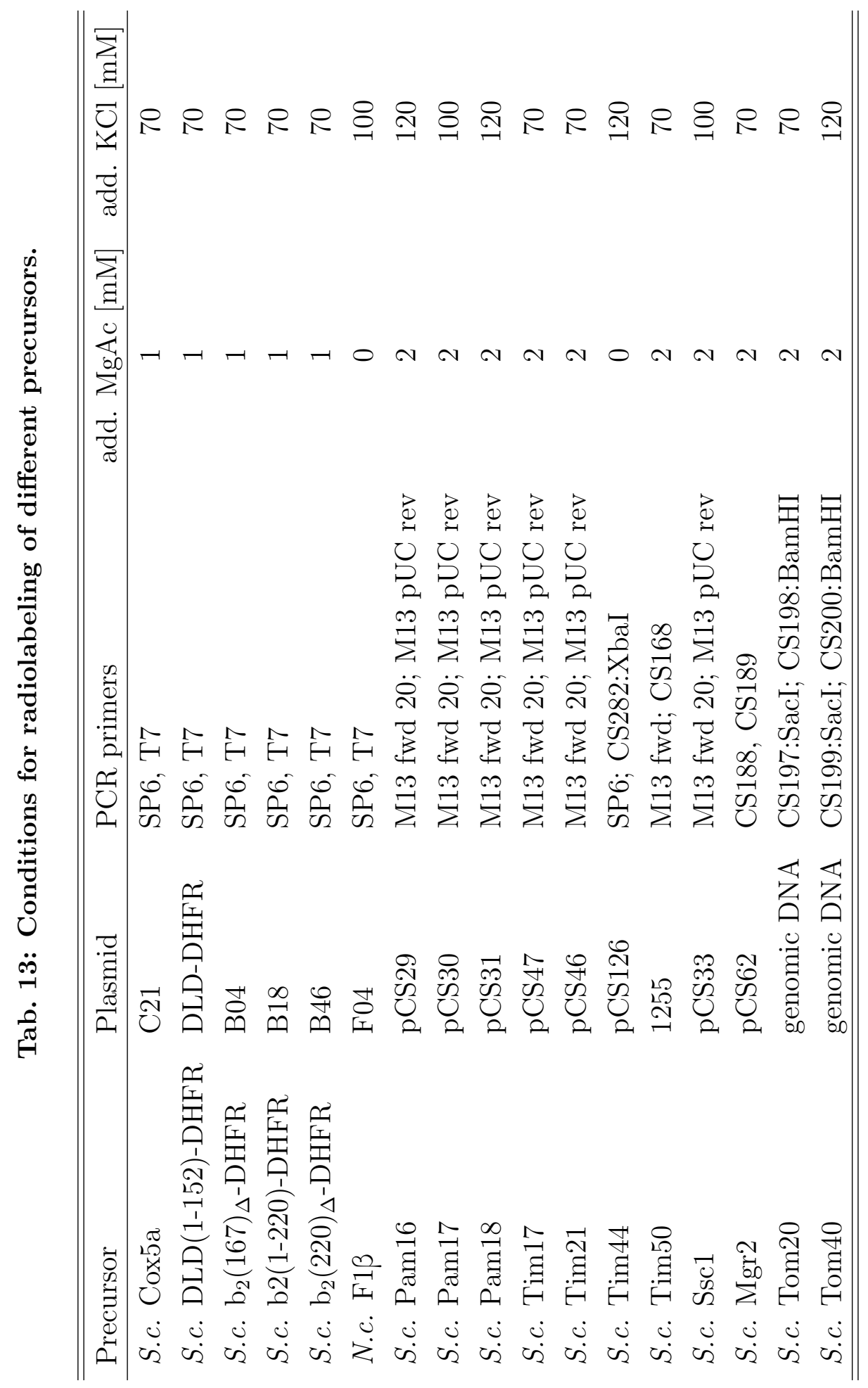




\subsubsection{Protein biochemistry}

\subsubsection{SDS-PAGE}

Separation of proteins by SDS polyacrylamid gel electrophoresis (SDS-PAGE) was carried out similar to Laemmli (1970). Briefly, separating gels were prepared with different acrylamid concentrations (16-10\%) using 30\%/ 0.8\% acrylamid/ bis-acrylamid (Gel Mix 30, Roth), $386 \mathrm{mM}$ Tris/HCl pH 8.8, 0.1\% SDS, 0.0588\% APS and 0.0588\% TEMED. The stacking gel was prepared with $5 \%$ acrylamid, $80 \mathrm{mM}$ Tris/HCl pH 6.8, $0.1 \%$ SDS, $0.1 \%$ APS and $0.2 \%$ TEMED. The running buffer contained $25 \mathrm{mM}$ Tris, $191 \mathrm{mM}$ glycine and $0.1 \%$ SDS. Gels were run in the MINI-Protean II (BioRad) system (300 V, $30 \mathrm{~mA}$ per gel) or in custom-made midi chambers (180 V, $35 \mathrm{~mA}$ per gel).

For better separation of proteins smaller than $6 \mathrm{kDa}$ urea was supplemented to the gels as suggested by Schägger (2006) for Tricine gels. The separating gel consisted of $17 \%$ acrylamid (using a $60 \% / 0.8 \%$ acrylamid/ bis-acrylamid mixture), $683 \mathrm{mM}$ Tris/HCl pH 8.8, $7.77 \mathrm{mM} \mathrm{NaCl}, 5.4 \mathrm{M}$ urea, 0.1\% SDS, 0.032\% APS and 0.066\% TEMED. The stacking gel contained $5.4 \%$ acrylamid (using a $60 \% / 0.8 \%$ acrylamid/ bis-acrylamid mixture), $108 \mathrm{mM}$ Tris/HCl pH 6.8, 3.3 M urea, 0.12\% SDS, 0.126\% APS and $0.11 \%$ TEMED. The running buffer contained $50 \mathrm{mM}$ Tris, $192 \mathrm{mM}$ glycine and $0.1 \%$ SDS. Gels were run in the MINI-Protean II (BioRad) system (250 V, $30 \mathrm{~mA}$ per gel) or in custommade midi chambers $(180 \mathrm{~V}, 30 \mathrm{~mA}$ per gel).

SDS sample buffer contained 2\% SDS, $10 \%$ glycerol, $60 \mathrm{mM}$ Tris/HCl pH 6.8, $1 \%$ $\beta$-mercaptoethanol and $0.01 \%$ bromphenolblue, a $4 \mathrm{x}$ stock was usually used.

The unstained SDS PAGE protein marker 6.4 - $200 \mathrm{kDa}$ (SERVA) was used as a molecular weight standard.

\subsubsection{BN-PAGE}

Native separation of protein complexes by blue native polyacrylamide gel electrophoresis (BN-PAGE) (Schägger and von Jagow, 1991) was performed as described in Dekker et al. (1997). The SE600 Ruby system (Hoefer, GE Healthcare) was used for casting and running the gels. Separating gels contained $1 \mathrm{x}$ gel buffer $(66.6 \mathrm{mM} \varepsilon$-amino n-caproic acid and $50 \mathrm{mM}$ Bis-Tris/HCl pH 7.0), acrylamide from a $48 \% / 1.5 \%$ acrylamide/ bisacrylamide stock, $0.44 \%$ APS and TEMED. The stacking gel contained 1x gel buffer, $3.84 \%$ acrylamide, $0.08 \%$ APS and $0.133 \%$ TEMED. For separation of the TIM23 complex a 
3-14\% gradient was used for the separation gel with the $14 \%$ part containing $20 \%$ glycerol. The cathode buffer contained $50 \mathrm{mM}$ Tricine, $15 \mathrm{mM}$ Bis-Tris and $0.02 \%$ Coomassie G250. For subsequent western blot the cathode buffer was exchanged after $1 / 3$ of the run against Coomassie-free buffer. The anode buffer contained $50 \mathrm{mM}$ Bis-Tris/ $\mathrm{HCl} \mathrm{pH}$ 7.0. The samples were prepared by resuspending mitochondria in solubilization buffer $(1 \%$ digitonin, $20 \mathrm{mM}$ Tris/HCl pH 7.4, $0.1 \mathrm{mM}$ EDTA, $50 \mathrm{mM} \mathrm{NaCl}, 10 \%$ glycerol and $1 \mathrm{mM}$ $\mathrm{PMSF}$ ) to $1 \mathrm{\mu g} / \mathrm{\mu L}$ and solubilized for $30 \mathrm{~min}$ on ice. After removal of insoluble material by centrifugation for $15 \mathrm{~min}$ at $20200 \mathrm{xg}, 4^{\circ} \mathrm{C}$ the supernatant was mixed with the appropriate volume of chilled 10x BN-loading dye (5\% Coomassie G250, $500 \mathrm{mM} \varepsilon$-amino n-caproic acid and $100 \mathrm{mM}$ Bis-Tris/HCl pH 7.0). After a short clarifying spin (5 min, $20200 \mathrm{xg}, 4^{\circ} \mathrm{C}$ ) the supernatant was loaded on the gel. The gel run was performed at $15 \mathrm{~mA}$ per gel at $100 \mathrm{~V}$ (overnight) or $600 \mathrm{~V}$ (during day) at $4^{\circ} \mathrm{C}$. The HMW calibration kit (GE Healthcare) was used as a molecular weight standard.

\subsubsection{Ponceau S staining of PVDF membranes}

In order to use fluorescently labeled secondary antibodies for detection the PVDF membranes were stained with Ponceau S staining solution $(0.1 \%(\mathrm{w} / \mathrm{v})$ Ponceau $\mathrm{S}$ and $5 \%$ $(\mathrm{w} / \mathrm{v})$ acetic acid $)$ ) for $5 \mathrm{~min}$ shaking. Destaining was achieved with $\mathrm{dH}_{2} \mathrm{O}$.

\subsubsection{Coomassie staining of gels and PVDF membranes}

Gels and membranes were stained with coomassie (40\% ethanol, $10 \%$ acetic acid and $0.15 \%$ Coomassie R250) and destained (30\% ethanol and $10 \%$ acetic acid) until protein bands could be clearly distinguished from the background.

Colloidal coomassie staining for subsequent mass spectrometric analysis was performed according to Neuhoff et al. $(1985,1988)$. To this end, the gel was fixed in $40 \%$ ethanol and $10 \%$ acetic acid for at least $60 \mathrm{~min}$ at room temperature shaking in a glass container. Subsequently, the gel was washed three times for $10 \mathrm{~min}$ with milli-Q water and then stained using four parts dye stock solution $(0.1 \%$ (w/v) Coomassie Brilliant Blue G250, $2 \%$ ortho-phosphoric acid and $10 \%$ ammonium sulfate) and one part methanol. Staining was carried out overnight at room temperature. Destaining was achieved by using $1 \%$ acetic acid. 


\subsubsection{Autoradiography}

Before exposing gels for autoradiography, the gels were stained with Coomassie and covered with a plastic bag on one side and two blotting papers (BF2 grade, $190 \mathrm{~g} / \mathrm{m}^{2}$, Sartorius stedim) on the other side. The gels were dried using a vacuum gel drier (Scie-Plas) at $65{ }^{\circ} \mathrm{C}$ for $1.5 \mathrm{~h}$ (mini gel) or $2.5 \mathrm{~h}$ (midi gel, BN gel). Subsequently the protein size standard was marked with radioactive ink (standard fountain pen ink containing $100 \mu \mathrm{Ci}$ ${ }^{35} \mathrm{~S}-$ Met per cartridge) and covered with adhesive tape. The gels were exposed to Storage Phosphor Screens (GE Healthcare). After exposure the signals were digitized using the STORM820 scanner (GE Healthcare). Quantification was performed using the ImageQuantTL software (GE Healthcare) with rolling ball background subtraction.

\subsubsection{Western blot}

Western blotting was carried out according to standard protocols Gallagher et al. (2004). PVDF membranes (Immobilon-P, Merck Millipore) were rinsed in methanol to activate them. Blotting papers (three on each side, see 5.2.3.5), PVDF membrane and the gel were soaked in transfer buffer (20 mM Tris, $150 \mathrm{mM}$ glycine, $0.02 \%$ SDS and $20 \%$ methanol), and the blot was assembled on semi dry chambers (Peqlab). Blotting was performed at $250 \mathrm{~mA}, 25 \mathrm{~V}$ (per midi SDS or blue native gel) for $2 \mathrm{~h}$.

\subsubsection{Immunodetection}

Immunodetection was carried out according to standard procedures (Gallagher et al., 2004). After completely destaining the PVDF membranes with methanol, they were blocked using $5 \%$ milk powder in TBS-T (20 mM Tris/HCl pH 7.5, $62 \mathrm{mM} \mathrm{NaCl}, 0.1 \%$ Tween 20) for $1 \mathrm{~h}$ at room temperature. For BN-PAGE blots $10 \%$ milk powder in TBS-T was used. Subsequently, the blots were briefly rinsed with TBS-T and then incubated in primary antibody solution (usually prepared as a dilution of rabbit serum in $5 \%$ milk powder in TBS-T or in TBS-T) for $1 \mathrm{~h}$ at room temperature or overnight at $4{ }^{\circ} \mathrm{C}$. Afterwards the blot was washed three times $10 \mathrm{~min}$ with TBS-T, incubated with secondary antibody (HRP coupled: 1:10000 in TBS-T, fluorescently labeled: 1:5000 in TBS-T) for $1 \mathrm{~h}$ at room temperature and washed three more times with TBS-T for $10 \mathrm{~min}$. The remaining liquid was removed from the blot and ECL solution was added (GE Healthcare) and signals were detected using x-ray films. For fluorescent detection the washed blots 
were scanned using a FLA-9000. For detection of biotin, the membranes were washed extensively after with TBS-T for $30 \mathrm{~min}$ after blocking. Streptavidin-HRP (1:1000) was diluted in TBS-T and incubated with the membrane. Subsequent processing was carried out as described above.

\subsubsection{Protein purification}

Expression conditions and purification strategies for the proteins used in functional assays in this study are listed in Table 14. All purification steps were performed on ice or at $\max .8^{\circ} \mathrm{C}$. Proteins were stored in small aliquots at $-20^{\circ} \mathrm{C}$.

Cell lysis was achieved by running the cell suspension $(0.1 \mathrm{~g} / \mathrm{mL}$, for all proteins containing one protease inhibitor pill (EDTA free) per $50 \mathrm{~mL}$ suspension) three times through a EmulsiFlex-C3 homogenizer (Avestin) operated at $100 \mathrm{MPa}$, subsequently the homogenate was centrifuged for $30 \mathrm{~min}$ at $39000 \mathrm{xg}$ and the supernatant filtered through $0.20 \mu \mathrm{m}$ filter (Sartorius). The supernatant was then loaded on the respective column using the ÄKTA purifier, washed with lysis buffer lacking active ingredients (like protease inhibitors or detergents) until a stable base line was reached and eluted with a suitable buffer. For dialysis the protein was transferred into SnakeSkin dialysis membrane (7000MW cut-off) and stirred in an appropriate volume of dialysis buffer over night at $4{ }^{\circ} \mathrm{C}$. Purity and integrity of the proteins was assessed by SDS-PAGE and coomassie staining.

Cells that expressed $b_{2}(167)_{\Delta}$-DHFR were lysed in $(20 \mathrm{mM}$ MOPS/ KOH pH 8.0, $1 \mathrm{mM}$ EDTA, $1 \mathrm{mM}$ DTT, $0.25 \%$ Triton X-100 and $1 \mathrm{mM}$ PMSF loaded on a resource $\mathrm{S}$ at $0.5 \mathrm{~mL} / \mathrm{min}$ (1 $\mathrm{ml}$ column volume), washed at $1 \mathrm{~mL} / \mathrm{min}$ and eluted with $20 \mathrm{mM}$ MOPS/ $\mathrm{KOH} \mathrm{pH}$ 8.0, $1 \mathrm{mM}$ EDTA and $1 \mathrm{M} \mathrm{NaCl}$ using a $0-100 \%$ buffer B gradient over $20 \mathrm{~mL}$. The major fraction of the protein eluted around $300 \mathrm{mM} \mathrm{NaCl}$, was pooled and mixed 1:1 with $80 \%$ glycerol.

For Tim2 $3^{\text {IMS }}$ the lysis buffer contained $20 \mathrm{mM}$ NaPi pH 7.4, $500 \mathrm{mM} \mathrm{NaCl}, 20 \mathrm{mM}$ imidazole and $1 \mathrm{mM}$ PMSF. The sample was loaded on a $1 \mathrm{~mL}$ HisTrap column at $0.5 \mathrm{~mL} / \mathrm{min}$, washed at $1 \mathrm{~mL} / \mathrm{min}$ and eluted with buffer containing $20 \mathrm{mM} \mathrm{NaPi} \mathrm{pH} \mathrm{7.4,500} \mathrm{mM} \mathrm{NaCl}$ and $500 \mathrm{mM}$ imidazole. Dialysis was performed against $20 \mathrm{mM}$ Tris/ $\mathrm{HCl} \mathrm{pH} 8.0$ and $50 \mathrm{mM} \mathrm{NaCl}$.

For Tim50 ${ }^{\mathrm{IMS}}$ lysis was achieved in $20 \mathrm{mM}$ Tris/ $\mathrm{HCl} \mathrm{pH} 8.0,500 \mathrm{mM} \mathrm{NaCl}, 5 \mathrm{mM}$ imidazole, $0.2 \mathrm{mM}$ AEBSF, $1 \mathrm{ng} / \mathrm{\mu L}$ leupeptin and $1 \mathrm{mM}$ PMSF. The soluble material was loaded on a $1 \mathrm{~mL}$ HisTrap column as described for Tim $23^{\mathrm{IMS}}$ and eluted with $20 \mathrm{mM}$ 
Tris/ $\mathrm{HCl} \mathrm{pH} \mathrm{8.0,500} \mathrm{mM} \mathrm{NaCl}$ and $500 \mathrm{mM}$ imidazole. The protein was dialyzed against

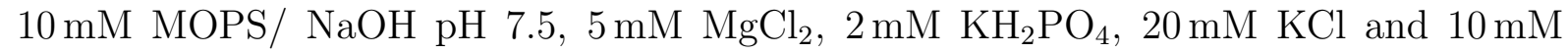
imidazole.

For Tim50 ${ }^{\triangle \mathrm{PBD}}$ lysis buffer contained $20 \mathrm{mM}$ NaPi pH 7.4, $100 \mathrm{mM} \mathrm{NaCl}, 5 \mathrm{mM}$ imidazole and $1 \mathrm{mM}$ PMSF, the supernatant was loaded on a $1 \mathrm{~mL}$ HisTrap and bound proteins eluted using $20 \mathrm{mM} \mathrm{NaPi} \mathrm{pH} \mathrm{7.4,} 200 \mathrm{mM} \mathrm{NaCl}, 500 \mathrm{mM}$ imidazole. The protein was dialyzed against $20 \mathrm{mM}$ Tris/ $\mathrm{HCl} \mathrm{pH} 8.0$ and $20 \mathrm{mM} \mathrm{NaCl}$.

Tim50 ${ }^{\mathrm{PBD}}$ lysis buffer contained $20 \mathrm{mM}$ Tris/ $\mathrm{HCl} \mathrm{pH} 8.0,500 \mathrm{mM} \mathrm{NaCl}, 10 \mathrm{mM}$ imidazole, $1 \mathrm{mM}$ PMSF, a $5 \mathrm{~mL}$ HisTrap column was used and elution was achieved with $20 \mathrm{mM}$ Tris/ $\mathrm{HCl} \mathrm{pH} \mathrm{8.0,500} \mathrm{mM} \mathrm{NaCl}$ and $500 \mathrm{mM}$ imidazole. The protein was dialyzed against Tris/ $\mathrm{HCl} \mathrm{pH} 8.0$ and $20 \mathrm{mM} \mathrm{NaCl}$, cleaved with $1 \mathrm{mg}$ TEV protease per $15 \mathrm{mg}$ protein for $2 \mathrm{~h}$ at $30^{\circ} \mathrm{C}$. The soluble material was run over a self-packed Ni-NTA column to remove the His-tagged protease as well as the cleaved tag and the flow-through was concentrated using centricons (5000-MW cut-off, Sartorius stedim) according to the manufactures instructions. This fraction was then loaded on a Superdex 75 16/60 HiLoad gelfiltration column operated at $1 \mathrm{~mL} / \mathrm{min}$. The protein containing fractions were pooled and concentrated.

For Tim $50^{\mathrm{C}}$ lysis was achieved in $20 \mathrm{mM}$ HEPES/ NaOH pH 8.0, $500 \mathrm{mM} \mathrm{NaCl}, 15 \mathrm{mM}$ imidazole and $1 \%$ Triton X-100. The supernatant was loaded on $1 \mathrm{~mL}$ HisTrap and eluted with $20 \mathrm{mM}$ HEPES/ NaOH pH 8.0, $500 \mathrm{mM} \mathrm{NaCl}, 500 \mathrm{mM}$ imidazole. Dialysis was carried out against $20 \mathrm{mM}$ HEPES/ NaOH pH 8.0 and $200 \mathrm{mM} \mathrm{NaCl}$. 


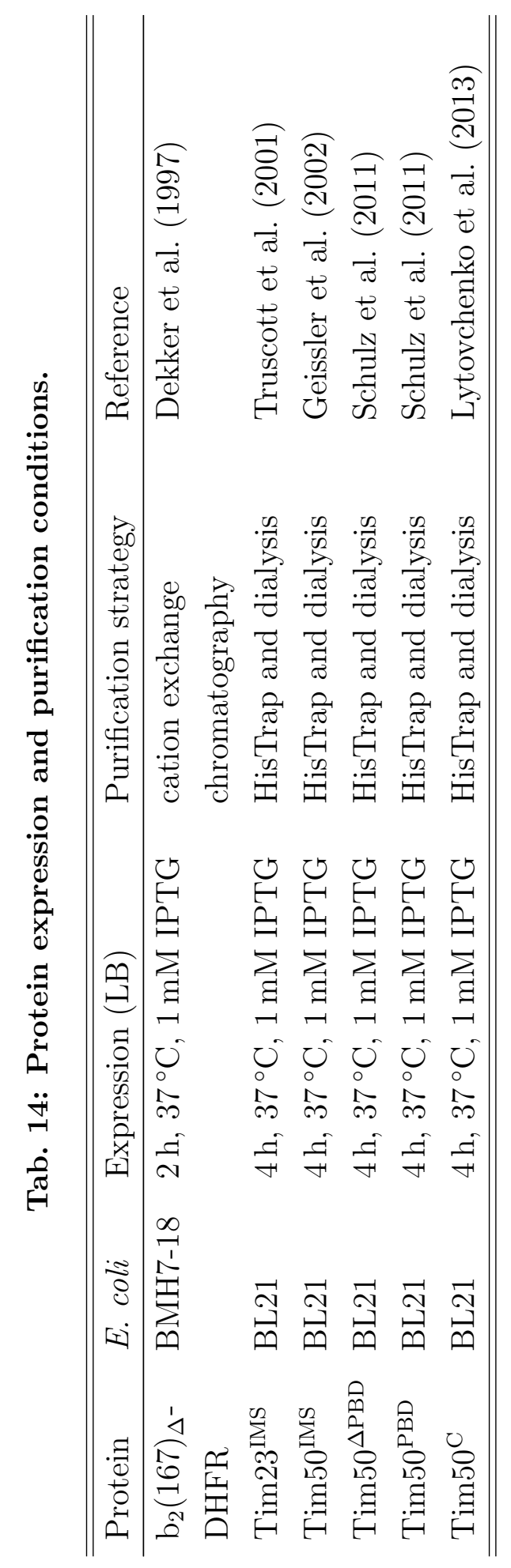




\subsubsection{Affinity purification of antisera}

In order to affinity purify the $\alpha$-Tim50 serum to obtain antibodies selectively recognizing Tim50 ${ }^{\mathrm{PBD}}$, purified PBD was immobilized on CNBr Sepharose. To this end $0.66 \mathrm{~g}$ speharose were hydrated in $130 \mathrm{~mL} 1 \mathrm{mM} \mathrm{HCl}$ for $15 \mathrm{~min}$ in a Büchner funnel. This resulted in $2-3 \mathrm{~mL} 50 \%$ slurry with a capacity of $5-10 \mathrm{mg} / \mathrm{mL}$. Subsequently, the slurry was washed with $2 \mathrm{~mL}$ coupling buffer (0.1 Msodium cabonate buffer $\mathrm{pH} 8.3,0.5 \mathrm{M} \mathrm{NaCl})$ and transferred into a falcon tube. $5.4 \mathrm{mg}$ purified Tim50 $0^{\mathrm{PBD}}$ were added to the sepharose in a reaction volume of $5 \mathrm{~mL}$. Crosslinking was performed at $4{ }^{\circ} \mathrm{C}$ over night. Afterwards, the slurry was transferred into the Büchner funnel and washed twice with $5 \mathrm{~mL}$ coupling buffer and twice with $5 \mathrm{~mL}$ blocking buffer (0.1 M Tris/ $\mathrm{HCl} \mathrm{pH} 8.0)$. Subsequently, the slurry was blocked in a falcon tube using $5 \mathrm{~mL}$ blocking buffer for $2 \mathrm{~h}$ at room temperature. Subsequent washes were performed by centrifugation $\left(5 \mathrm{~min}\right.$ at $400 \mathrm{xg}$ and $4{ }^{\circ} \mathrm{C}$ )

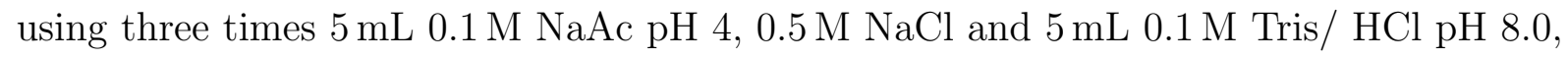
$0.5 \mathrm{M} \mathrm{NaCl}$. The slurry was packed into a column and stored in TBS (section 5.2.3.7) $0.02 \%$ sodium azide.

The purification of the antibodies was performed at room temperature. Initially, the column was washed with two times $5 \mathrm{~mL}$ TBS. Subsequently, $\alpha$-Tim50 serum was diluted 1:2 with TBS and run over the column for four times. After washing with three times $5 \mathrm{~mL}$ TBS, the bound antibodies were eluted using ten times $1 \mathrm{~mL} 0.1 \mathrm{M}$ glycine pH 2.8. The elution fractions were neutralized using $200 \mu \mathrm{L} 1 \mathrm{M}$ Tris base $\mathrm{pH} 11$ and supplemented with BSA to $0.1 \mathrm{mg} / \mathrm{mL}$. 1:200 dilution of the fractions were tested for the recognition of wild-type Tim50 as well as Tim50 ${ }^{\triangle \mathrm{PBD}}$. The affinity column was neutralized using $2 \mathrm{x}$ $5 \mathrm{~mL}$ TBS and was stored in TBS with azide as described above.

\subsubsection{Specialized assays}

\subsubsection{Protein import in mitochondria}

Import of radiolabeled precursor proteins $(\max 10 \%(\mathrm{v} / \mathrm{v}))$, recombinant protein or peptides into mitochondria was carried out according to Ryan et al. (2001). The reactions were performed in import buffer $(250 \mathrm{mM}$ sucrose, $10 \mathrm{mM}$ MOPS/ KOH pH 7.2, $80 \mathrm{mM}$ $\mathrm{KCl}, 2 \mathrm{mM} \mathrm{KH} \mathrm{PO}_{4}, 5 \mathrm{mM} \mathrm{MgCl} 2,5 \mathrm{mM}$ methionine and $1 \%$ fatty-acid free BSA) supplemented with $2 \mathrm{mM}$ ATP and NADH at $25^{\circ} \mathrm{C}$. Creatine kinase (CK, $0.1 \mathrm{mg} / \mathrm{mL}$ ) and 
creatine phosphate $(\mathrm{CP}, 5 \mathrm{mM})$ were added as an ATP regenerating system when import was performed longer than $30 \mathrm{~min}$. The membrane potential was dissipated using $1 \mu \mathrm{M}$ valinomycin, $8 \mu \mathrm{M}$ antimycin A and $20 \mu \mathrm{M}$ oligomycin (AVO). Unimported precursor proteins were removed by treatment with $50 \mathrm{mg} / \mathrm{L}$ proteinase K. After inactivation of proteinase K using $2 \mathrm{mM}$ PMSF, mitochondria were pelleted by centrifugation for $10 \mathrm{~min}$ at $12000 \mathrm{xg}$ and $4^{\circ} \mathrm{C}$, washed with SEM buffer (see 5.2.1.7) and analyzed by BN-PAGE or SDS-PAGE in conjunction with autoradiography or Western blot. For competition of precursor import with different peptides, mitochondria were preincubated with the indicated concentration of peptide for $2 \mathrm{~min}$ at $25^{\circ} \mathrm{C}$.

In order to inhibit the import using affinity purified antibodies, mitochondria were incubated for $20 \mathrm{~min}$ on ice in $10 \mathrm{mM}$ MOPS pH 7.4 to convert them to mitoplasts. After addition of the respective antibodies and incubation on ice for $15 \mathrm{~min}$, mitochondria were reisolated by centrifugation, resuspended in import buffer and used for import.

\subsubsection{Inward driving activity}

The inward driving activity of the import motor was assayed according to Frazier et al. (2004). To this end, $40 \mu \mathrm{g}$ isolated mitochondria were diluted in import buffer to $0.8 \mu \mathrm{g} / \mathrm{\mu L}$ and mixed with $2 \mathrm{mM}$ ATP, $2 \mathrm{mM} \mathrm{NADH}, 5 \mathrm{mM} \mathrm{CP}, 0.1 \mathrm{mg} / \mathrm{mL}$ and $5 \mu \mathrm{M}$ MTX. Radiolabeled $\mathrm{b}_{2}(220)$-DHFR was mixed with $10 \mu \mathrm{M}$ methotrexate (MTX), incubated for 5 min on ice and imported into the prewarmed $\left(2 \min\right.$ at $\left.25^{\circ} \mathrm{C}\right)$ reaction $(\max .10 \%(\mathrm{v} / \mathrm{v}))$. After import for $15 \mathrm{~min}$ the membrane potential was dissipated using $1 \mu \mathrm{M}$ valinomycin and the reaction was split into equal parts. One part was directly transferred to ice, the other was incubated for different times $(\Delta \mathrm{t})$ at $25^{\circ} \mathrm{C}$ and subsequently PK treated $(4 \mu \mathrm{g} / \mathrm{mL}$, $10 \mathrm{~min}$ on ice). After inactivation of the PK (4 mM PMSF, $10 \mathrm{~min}$ on ice) the samples were washed with SEM, resuspended in protein loading dye and analyzed by SDS-PAGE and autoradiography.

\subsubsection{Generation of transport intermediate arrested in the TOM complex}

In order to test the ability of mutant mitochondria to generate intermediates at the TOM complex, radiolabeled Su9-DHFR was arrested, as described by Kanamori et al. (1999). Therefore, isolated mitochondria ( $0.5 \mathrm{~g} / \mathrm{L})$ were incubated in $10 \mathrm{mM} \mathrm{MOPS} /$ $\mathrm{KOH} \mathrm{pH} 7.2,250 \mathrm{mM}$ sucrose, $10 \mathrm{mM} \mathrm{KCl}, 5 \mathrm{mM} \mathrm{MgCl} 2,2 \mathrm{mM}$ methionine and $10 \mu \mathrm{M}$ 
carbonyl cyanid 3-chlorophenylhydrazon (CCCP) with radiolabeled Su9-DHFR on ice for 15 min. Subsequently, mitochondria were diluted 1:5 using $10 \mathrm{mM}$ MOPS/ KOH pH 7.2, $250 \mathrm{mM}$ sucrose, $10 \mathrm{mM} \mathrm{KCl}, 20 \mu \mathrm{M}$ CCCP, $0.5 \mu \mathrm{M}$ MTX, $1 \mathrm{mM}$ NADPH and reisolated by centrifugation. The pellet was resuspended in the same buffer $(0.5 \mathrm{~g} / \mathrm{L})$ and the sample was split into two, one half was proteinase K $(100 \mathrm{mg} / \mathrm{L})$ treated. The supernatant of the proteinase $\mathrm{K}$ treated sample was precipitated using $15 \%$ TCA (described in section 5.2.1.6), while the mitochondrial pellet of the untreated sample was used directly. Samples were analyzed by SDS-PAGE and autoradiography.

\subsubsection{Generation of transport intermediate arrested in the TOM-TIM23 supercomplex}

The translocation supercomplex comprising the TOM and TIM23 complex as well as the substrate $\mathrm{b}_{2}(167)_{\Delta}$-DHFR was generated using purified substrate that was imported in vitro into isolated mitochondria according to Dekker et al. (1997). To this end, mitochondria were suspended at $1 \mathrm{\mu g} / \mathrm{\mu L}$ in import buffer containing BSA, supplemented with $2 \mathrm{mg}$ ATP and NADH, $5 \mathrm{mg}$ creatine phosphate, $0.1 \mathrm{mg} / \mathrm{mL}$ creatine kinase and $1 \mu \mathrm{M}$ MTX. Import was performed with $0.5 \mathrm{nmol} / \mathrm{mg}$ substrate/ mitochondria for semi-saturating and $1.5 \mathrm{nmol} / \mathrm{mg}$ for saturating conditions for $15 \mathrm{~min}$ at $25^{\circ} \mathrm{C}$ under slight agitation. Optionally, reactions were stopped with AVO mix and subsequently processed for BN-PAGE, complex isolations or further protein imports as described in the respective sections.

\subsubsection{Photo-Crosslinking}

For in organello photo-crosslinking, mitochondria were mixed in import buffer $(1 \mathrm{~g} / \mathrm{L})$ lacking BSA with $2 \mu \mathrm{M}$ photopeptide and incubated for $10 \mathrm{~min}$ on ice. UV-irradiation was performed for $30 \mathrm{~min}$ on ice using an Ultratech $400 \mathrm{~W}$ halogen metal vapor lamp (Osram) and a B270 glass screen (Schott, Mainz, Germany) to filter protein-damaging wavelengths below $300 \mathrm{~nm}$ (Jahn et al., 2002). Subsequently, mitochondria were washed with SEM buffer and analyzed by Western-blotting.

For isolation of photo-adducts, mitochondria were resuspended $(10 \mathrm{~g} / \mathrm{L})$ in lysis buffer (100 mM Tris/ HCl pH 8.0, $8 \mathrm{M}$ urea, $1 \%$ SDS, $2 \%$ Triton X-100, 1 mM EDTA, $200 \mathrm{mM}$ $\mathrm{NaCl}$ and $1 \mathrm{mM} \mathrm{PMSF}$ ) and incubated for $10 \mathrm{~min}$ at room temperature. Samples were diluted to $1 \mathrm{~g} / \mathrm{L}$ with dilution buffer (lysis buffer lacking SDS and containing $0.8 \mathrm{M}$ 
urea, $2 \mathrm{mg} / \mathrm{L}$ leupeptin and $2 \mathrm{mM}$ 4-(2-Aminoethyl) benzenesulfonyl fluoride hydrochloride $(\mathrm{AEBSF})$ ) to reduce the concentration of SDS and urea and incubated $10 \mathrm{~min}$ at $4{ }^{\circ} \mathrm{C}$. After this solubilization and removal of insoluble particles by centrifugation for $10 \mathrm{~min}$ at $12000 \mathrm{xg}$, the sample was loaded onto streptavidin agarose (Thermo Scientific). Binding was carried out for $5 \mathrm{~h}$ at $4^{\circ} \mathrm{C}$ mixing the sample head-over-head. After washing the columns with wash buffer A (as lysis buffer, but with $2 \%$ SDS), wash buffer B (as lysis buffer, but with $0.2 \% \mathrm{SDS}$ and $1 \mathrm{M} \mathrm{NaCl})$ and wash buffer $\mathrm{C}(100 \mathrm{mM}$ Tris/ $\mathrm{HCl} \mathrm{pH}$ 8.0, $0.2 \%$ Triton X-100 and $1 \mathrm{mM}$ EDTA), elution was achieved by incubation at $95^{\circ} \mathrm{C}$ for $15 \mathrm{~min}$ in protein loading buffer (PLD, $2 \%$ SDS, $10 \%$ glycerol, $60 \mathrm{mM}$ Tris/ $\mathrm{HCl} \mathrm{pH}$ 6.8, $0.01 \%$ bromphenoleblue and $1 \% \beta$-mercaptoethanol). Samples of the soluble fraction loaded on the affinity column, unbound and eluate were analyzed by SDS-PAGE and western blot.

Photo-crosslinking of purified proteins was performed using a 0.5-1.5 molar ratio of protein to peptide. The sample was equilibrate on ice for 15 min before crosslinking using the aforementioned UV lamp for 30 min on ice. Samples were analyzed by SDS-PAGE and western blotting or by colloidal coomassie staining and MS/ MS analysis.

\subsubsection{Chemical Crosslinking}

For in organello DFDNB crosslinking $1 \mathrm{~g} / \mathrm{L}$ mitochondria were suspended in $20 \mathrm{mM}$ HEPES pH 7.2 and $100 \mathrm{mM} \mathrm{NaCl}$ and incubated with $20 \mu \mathrm{M}$ presequence peptide for $15 \mathrm{~min}$ at $25^{\circ} \mathrm{C}$. Subsequent to crosslinking with $1 \mathrm{mM}$ DFDNB for $30 \mathrm{~min}$ on ice the reaction was stopped by quenching with $250 \mathrm{mM}$ Tris/HCl pH 7.4. Afterwards the mitochondria were reisolated and washed with $20 \mathrm{mM}$ HEPES pH 7.2, $80 \mathrm{mM} \mathrm{KCl}$ and $600 \mathrm{mM}$ sorbitol. The samples were analyzed by SDS-PAGE and western blotting.

Chemical crosslinking of purified components was carried out using $1 \mu \mathrm{M}$ of protein mixed with the indicated amount of peptide or additional protein for $15 \mathrm{~min}$ on ice in buffer containing $20 \mathrm{mM}$ HEPES, pH 7.2 and $100 \mathrm{mM} \mathrm{NaCl}$. $100 \mu \mathrm{M}$ DFDNB was used to crosslink for $30 \mathrm{~min}$ on ice and subsequently the reaction was quenched for $15 \mathrm{~min}$ with $140 \mathrm{mM}$ Tris/ $\mathrm{HCl} \mathrm{pH} 7.5$ and $5 \% \beta$-mercaptoethanol on ice. The samples were analyzed by SDS-PAGE and western blotting. 


\subsubsection{Surface plasmon resonance}

Surface plasmon resonance was measured with a Reichert SPR Biosensor SR7000DC using a $\mathrm{Ni}^{2+}$ chelator sensorchip NiHC500m (XanTec bioanalytics). The buffer system contained $50 \mathrm{mM}$ HEPES, pH 7.4, $150 \mathrm{mM} \mathrm{NaCl}$, and $50 \mathrm{mM}$ EDTA $\left(20^{\circ} \mathrm{C}\right)$ (flow: $\left.40 \mathrm{~mL} / \mathrm{min}\right)$. Tim50 IMS , Tim50 ${ }^{\mathrm{PBD}}$ or Tim50 ${ }^{\mathrm{C}}\left(250 \mathrm{nM}\right.$ in buffer) were immobilized on the $\mathrm{Ni}^{2+}$ loaded channel of the chip at a flow rate of $30 \mathrm{~mL} / \mathrm{min}$ to $2500-3500$ response units. The second channel was used as a reference. Increasing concentrations of pALDH in running buffer were injected for $270 \mathrm{~s}$ to both channels and the dissociation was followed for $12 \mathrm{~min}$. The response difference between sample and reference channels was recorded. Kinetic data analysis was performed using Scrubber 2.0 (BioLogic Software). The response was referenced to buffer injections and normalized using the molecular weight (in $\mathrm{kDa}$ ) of the ligand. Recordings were performed with the help of Dr. O. Lytovchenko (Institute of Cellular Biochemistry, Göttingen, Germany) and M. Kilisch (Institute of Molecular Biology, Göttingen, Germany).

\subsubsection{Mass spectrometic analysis of photoadducts}

Colloidal coomassie stained protein bands of SDS polyacrylamid gels were excised and in-gel digest using trypsin was performed. To this end the gel pieces were washed with $25 \mathrm{mM} \mathrm{NH}_{4} \mathrm{HCO}_{3} /$ water, $25 \mathrm{mM} \mathrm{NH} \mathrm{NHCO}_{3} / 50 \%$ acetonitrile and $100 \%$ acetonitrile. Subsequently, disulfides were reduced with $10 \mathrm{mM}$ dithiothreitol, $25 \mathrm{mM} \mathrm{NH}_{4} \mathrm{HCO}_{3} /$ water for $1 \mathrm{~h}$ at $56^{\circ} \mathrm{C}$. After washing the gel piece as described above and performing carbamidomethylation using $25 \mathrm{mM}$ iodoacetoamide in $25 \mathrm{mM} \mathrm{NH}_{4} \mathrm{HCO}_{3} /$ water the ingel digest was carried out using $120 \mathrm{ng}$ trypsin at $37^{\circ} \mathrm{C}$ overnight. Peptide extraction was achieved with $0.1 \%$ trifluoroacetic acid (TFA). Subsequently, peptides were separated by reverse-phase chromatography (EASY-nLC; Bruker Daltonics) using a C18 column (PepMap 100 C18 nano-column; Dionex) and an 9.5\%-90\% acetonitrile gradient (in $0.1 \%$ TFA) for $80 \mathrm{~min}$.

The matrix used for MALDI was $\alpha$-cyano-4-hydroxycinnamic acid (HCCA) $(4.5 \%$ satured $\mathrm{HCCA}$ in $90 \%$ acetonitrile, $0.1 \%$ TFA and $1 \mathrm{mM} \mathrm{NH}_{4} \mathrm{H}_{4} \mathrm{PO}_{4}$ ). It was mixed with the eluate of the reverse-phase chromatography and spotted automatically on a target (Proteineer fc II; Bruker Daltonics). MALDI-TOF/TOF analysis was carried out using a Ultraflextreme setup (Bruker Daltonics), which recorded MS as well as post-source 
decay MS/MS. Different software packages were used for data analysis and interpretation (WARP-LC, AutoXecute, Flex-Analysis and Biotools; Bruker Daltonics). Candidate spectra matching a calculated crosslink between tryptic fragments of a target protein and a photopeptide were evaluated by hand to verify the position of the crosslink position. Sample preparation and analysis with MALDI-TOF/TOF was performed by Olaf Bernhard and Dr. Bernhard Schmidt (Institute of Cellular Biochemistry, University of Göttingen).

Linear MALDI (MALDI-TOF-MS) was performed using protein-photopeptide complexes in solution. To this end, the reaction was desalted using C4 ZipTips (Millipore) and bound proteins were eluted with $80 \%$ acetonitrile and $0.1 \%$ TFA. Dried droplets were spotted on steel targets using $1 \mu \mathrm{L}$ of eluate and $2 \mu \mathrm{L}$ HCCA matrix solution (Schulz et al., 2011). MALDI-TOF analysis was carried out using a Ultraflex setup (Bruker Daltonics) in linear mode (m/z range of 5,000-25,000) using an external standard. FlexAnalysis 3.3 software (Bruker Daltonics) was used for data analysis and interpretation. Sample preparation and analysis of MALDI-TOF-MS was performed in the Proteomics group of Dr. Olaf Jahn (Max-Planck-Institute for Experimental Medicine, Göttingen).

\subsubsection{HA co-immunoprecipitations}

For co-immunoprecipitations using monoclonal antibodies ( $\alpha$-HA (Sigma Aldrich) or $\alpha$ $\mathrm{His}_{6}$ (Clonetech)), $15 \mu \mathrm{g}$ of antibodies were bound to $50 \mu \mathrm{L} 50 \%$ Dynabeads ProteinG (Invitrogen Dynal) in $200 \mu \mathrm{L}$ PBS for 20 min while rotating. After placing the mixture on a magnet, the supernatant was removed and the beads were washed with PBS. Precipitation conditions were used according to Mokranjac et al. (2003a). Briefly, mitochondria were resuspended in solubilization buffer $(50 \mathrm{mM} \mathrm{NaPi} \mathrm{pH}$ 7.4, $100 \mathrm{mM} \mathrm{NaCl}, 10 \%$ glycerol, $1 \%$ digitonin and $1 \mathrm{mM}$ PMSF) at $2 \mu \mathrm{g} / \mu \mathrm{L}$ and mixed for $30 \mathrm{~min}$ at $4{ }^{\circ} \mathrm{C}$. Insoluble material was pelleted by centrifugation for $10 \mathrm{~min}$ at $12000 \mathrm{xg}$ and $4{ }^{\circ} \mathrm{C}$. The supernatant was loaded on the Dynabeads and samples were incubated rotating for $2 \mathrm{~h}$ at $4^{\circ} \mathrm{C}$. Subsequently, samples were washed with solubilization buffer containing $0.3 \%$ digitonin and eluted with protein loading dye without reducing agent. 


\subsubsection{Immobilization of antibodies on protein A sepharose}

When serum was used for co-immunoprecipitations, it was coupled to to ProteinA sepharose (GE Healthcare) (Hutu et al., 2008). To this end, $100 \mu \mathrm{L}(50 \%)$ sepharose was washed with 0.1 M KPi pH 7.4 in Mobicols (MoBiTec). Washing was performed by centrifugation for $30 \mathrm{~s}$ at $100 \mathrm{xg}$ and $4^{\circ} \mathrm{C}$. Subsequently, $200 \mu \mathrm{L}$ serum were diluted with $200 \mu \mathrm{L}$ $0.1 \mathrm{M} \mathrm{KPi} \mathrm{pH} 7.4$ and incubated with the sepharose beads for $1 \mathrm{~h}$ at room temperature, rotating. Afterwards, the beads were washed with $0.1 \mathrm{M}$ sodium borate, and antibodies were crosslinked to the support by incubation in $0.1 \mathrm{M}$ sodium borate containing $5 \mathrm{mg} / \mathrm{mL}$ dimethyl pimelimidate (Thermo Scientific) for $30 \mathrm{~min}$ at room temperature, rotating. The reaction was quenched by washing with $1 \mathrm{M}$ Tris $\mathrm{pH} 7.4$ and incubation in the same buffer for at least $2 \mathrm{~h}$ at room temperature. Finally, the columns were washed with $0.1 \mathrm{M}$ glycine $\mathrm{pH} 2.8$, neutralized with TBS and stored in TBS with $2 \mathrm{mM}$ sodium azide at $4{ }^{\circ} \mathrm{C}$.

\subsubsection{1 $\alpha$-Tim23 co-immunoprecipitation}

For standard $\alpha$-Tim23 immunoprecipitation the following conditions were: $20 \mathrm{mM}$ Tris/ $\mathrm{HCl} \mathrm{pH} \mathrm{7.4,} 50 \mathrm{mM} \mathrm{NaCl}, 0.5 \mathrm{mM}$ EDTA, $10 \%$ glycerol, $2 \mathrm{mM} \mathrm{PMSF}$ and $1 \%$ digitonin for solubilization of isolated mitochondria $(1 \mu \mathrm{g} / \mu \mathrm{L})$ for $30 \mathrm{~min}$ on ice. The soluble material was bound to the antibody loaded ProteinA sepharose for $1.5 \mathrm{~h}$ mixing head-over-head at $4{ }^{\circ} \mathrm{C}$. The columns were washed using solubilization buffer containing $1 \mathrm{mM}$ PMSF and $0.3 \%$ digitonin. Samples were eluted using $100 \mathrm{mM}$ glycine $\mathrm{pH} 2.8$, neutralized with Tris base and subsequently precipitated with (15\%) TCA.

Co-immunoprecipitations under conditions lacking or containing ATP were performed according to Voisine et al. (1999). To this end, mitochondria $(1 \mathrm{mg} / \mathrm{mL})$ were solubilized in $30 \mathrm{mM}$ Tris/ $\mathrm{HCl} \mathrm{pH} \mathrm{7.4,} 80 \mathrm{mM} \mathrm{KCl,} 5 \mathrm{mM} \mathrm{MgCl}_{2}, 5 \%$ glycerol, $1 \mathrm{mM} \mathrm{PMSF}$ and $1 \%$ digitonin for $30 \mathrm{~min}$ on ice. For ATP containing reactions, $2 \mathrm{mM}$ ATP was added. Reactions lacking ATP contained $5 \mathrm{mM}$ EDTA instead. After removal of unsoluble material, the supernatant was loaded on $\alpha \operatorname{Tim} 23$ ProteinA sepharose for $1.5 \mathrm{~h}$ at $4{ }^{\circ} \mathrm{C}$. Columns were washed with the respective solubilization buffer containing $0.3 \%$ digitonin. Samples were eluted with $100 \mathrm{mM}$ glycine $\mathrm{pH} 2.8$ as described above. 


\subsubsection{Induction of temperature-sensitive phenotype}

Phenotypes of temperature-sensitive mutants were induced as described by Hutu et al. (2008). Resuspended mitochondria were incubated at $37^{\circ} \mathrm{C}$ for 15 min under slight agitation. For subsequent import analysis the mutants were transferred to room temperature and energizing components (ATP, NADH and optionally CK and CP) were added. For co-immunoprecipitations the heatshock was performed in solubilization buffer without detergent, which was added afterwards.

\subsubsection{Preparation of IgG sepharose}

Sepharose coupled with Hs IgG were prepared as described for the affinity columns (section 5.2.3.9). Briefly, 3.75 g CNBr actived sepharose were hydrated in $100 \mathrm{~mL} 1 \mathrm{mM} \mathrm{HCl}$ for $30 \mathrm{~min}$ at room temperature. After washing and equilibration, $50 \mathrm{mg}$ HsIgG were dissolved in coupling buffer without $\mathrm{NaCl}$ and incubated with the slurry in a reaction volume of $\sim 10 \mathrm{~mL}$ for $2 \mathrm{~h}$ at room temperature. The slurry was subsequently washed with the same buffer and incubated in blocking buffer over night at $4^{\circ} \mathrm{C}$. Finally, the slurry was washed with $4 \mathrm{x}$ coupling buffer containing $\mathrm{NaCl}, 2 \mathrm{x} 0.1 \mathrm{M}$ glycine $\mathrm{pH} 2.8,2 \mathrm{x} 0.2 \mathrm{M}$ glycine $\mathrm{pH}$

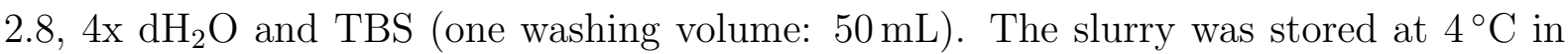
TBS containing azide.

\subsubsection{Tim23 ${ }^{\mathrm{PA}}$ isolation}

Isolation of the TIM23 complex using ProteinA tagged Tim23 was performed in the following way (Voisine et al., 1999; Geissler et al., 2002). First $1.1 \mathrm{mg}$ mitochondria, $2 \mathrm{mM}$ ATP, $2 \mathrm{mM}$ NADH, $5 \mathrm{mM}$ creatine phosphate and $0.1 \mathrm{mg} / \mathrm{mL}$ creatine kinase were mixed in import buffer containing BSA to $1 \mathrm{mg} / \mathrm{mL}$ mitochondria. Samples were incubated for $17 \mathrm{~min}$ at $25^{\circ} \mathrm{C}$, centrifuged for $10 \mathrm{~min}$ at $12000 \mathrm{xg}$ and $4^{\circ} \mathrm{C}$ and resuspended to $1 \mathrm{mg} / \mathrm{mL}$ in import buffer containing ATP, NADH, creatine phosphate and creatine kinase at the same concentrations. Following another incubation for $32 \mathrm{~min}$ at $25^{\circ} \mathrm{C}$, AVO mix was added to dissipate the membrane potential for $5 \mathrm{~min}$ at $25^{\circ} \mathrm{C}$. Mitochondria were pelleted and washed with SEM buffer. Subsequently, the mitochondria were resuspended to $1 \mathrm{mg} / \mathrm{mL}$ in solubilization buffer $(20 \mathrm{mM}$ Tris/ $\mathrm{HCl} \mathrm{pH} \mathrm{7.4,} 150 \mathrm{mM} \mathrm{NaCl}, 10 \%$ glycerol, $1 \mathrm{mM}$ EDTA, $1 \%$ digitonin and $2 \mathrm{mM}$ PMSF), solubilized on ice for $30 \mathrm{~min}$ and centrifuged for $15 \mathrm{~min}$ at $12000 \mathrm{xg}$ and $4{ }^{\circ} \mathrm{C}$. The supernatant was loaded on $60 \mu \mathrm{L} \mathrm{IgG}$ 
sepharose beads, mixed for $2 \mathrm{~h}$ rotating, and subsequently washed with solubilization buffer containing $0.3 \%$ digitonin. Samples were eluted with $100 \mathrm{mM}$ glycin pH 2.8, precipitated with $15 \%$ TCA (see section 5.2.1.6) and analyzed by SDS-PAGE and western blotting.

\subsubsection{Isolation of TOM-TIM23 supercomplex}

Isolation of the TOM-TIM23 supercomplex coupled with the arrested substrate $\mathrm{b}_{2}(167)_{\Delta^{-}}$ DHFR was carried out from isolated Tom $22^{\text {His }}$ mitochondria (Meisinger et al., 2001). To this end, semi-saturating amounts of MTX treated $b_{2}(167)_{\Delta}$-DHFR were imported (see 5.2.4.4), the mitochondria were washed with SEM buffer and then solubilized on ice at $1 \mathrm{\mu g} / \mathrm{\mu L}$ in $30 \mathrm{mM}$ Tris/ $\mathrm{HCl} \mathrm{pH} 7.4,150 \mathrm{mM} \mathrm{NaCl}, 10 \%$ glycerol, $0.1 \mathrm{mM}$ EDTA, $1 \%$ digitonin, $30 \mathrm{mM}$ imidazole and $1 \mathrm{mM}$ PMSF for $30 \mathrm{~min}$ at $4{ }^{\circ} \mathrm{C}$ rotating. Insoluble material was removed by centrifugation for $10 \mathrm{~min}$ at $4{ }^{\circ} \mathrm{C}$ and $12000 \mathrm{xg}$. The supernatant was loaded on NiNTA-agarose $(10 \mu \mathrm{L}$ bed volume per $250 \mu \mathrm{L}$ solubilizate) and inubated for $1 \mathrm{~h}$ mixing at $4{ }^{\circ} \mathrm{C}$. The beads were washed with solubilization buffer containing $0.3 \%$ digitonin and $80 \mathrm{mM}$ imidazole. Samples were eluted with washing buffer containing $200 \mathrm{mM}$ imidazole and analyzed by SDS-PAGE and western blotting.

\subsubsection{Tim23 pulldown}

Pulldown of mitochondrial interaction partners of Tim $23^{\mathrm{IMS}}$ was essentially performed as described by Geissler et al. (2002). Purified His-tagged Tim23 $3^{\mathrm{IMS}}$ was immobilized on Ni-NTA agarose. Mitochondria were solubilized using $20 \mathrm{mM}$ HEPES/KOH, pH 7.4, $100 \mathrm{mM} \mathrm{KOAc,} 10 \mathrm{mM} \mathrm{Mg}(\mathrm{OAc})_{2}, 10 \%$ (v/v) glycerol, $20 \mathrm{mM}$ imidazole, and $0.5 \%$ (v/v) Triton X-100 for $30 \mathrm{~min}$ at $4{ }^{\circ} \mathrm{C}$ to a final concentration of $1 \mu \mathrm{g} / \mu \mathrm{L}$. Insoluble material was removed by centrifugation for $15 \mathrm{~min}$ at $12.000 \mathrm{xg}$ and $4{ }^{\circ} \mathrm{C}$. The supernatant was loaded on the Tim $23^{\text {IMS }}$ charged Ni-NTA agarose beads for $1 \mathrm{~h}$ at $4{ }^{\circ} \mathrm{C}$. As a control empty beads were used. Subsequently the beads were washed with solubilization buffer containing $0.25 \%(\mathrm{v} / \mathrm{v})$ Triton X-100 and proteins were eluted using $8 \mathrm{M}$ urea containing protein loading dye. 


\subsubsection{Measurment of membrane potential}

The membrane potential across the inner mitochondrial membrane was assessed according to Geissler et al. (2000). Briefly, $500 \mu \mathrm{L} \Delta \psi$-buffer (600 mM sorbitol, 0.1\% BSA, $10 \mathrm{mM}$ $\mathrm{MgCl}_{2}$ and $20 \mathrm{mM} \mathrm{KPi} \mathrm{pH} \mathrm{7.2)} \mathrm{was} \mathrm{prepared} \mathrm{in} \mathrm{a} \mathrm{micro} \mathrm{quartz} \mathrm{cuvette.} 5 \mu \mathrm{L} \operatorname{DiSC}_{3}(5)$ dye solution (1:10 dilution of a saturated solution in ethanol) was added, mixed and fluorescence was measured in a F-7000 fluorospectrometer (Hitachi) as a time scan (excitation: $622 \mathrm{~nm}$, emission: $670 \mathrm{~nm}$, slit $5 \mathrm{~nm}$, response $0.1 \mathrm{~s}$ ). After $55 \mathrm{~s}, 5 \mu \mathrm{L}$ of a $4 \mathrm{mg} / \mathrm{mL}$ mitochondria suspension in SEM were added. After $200 \mathrm{~s}, 5 \mu \mathrm{L}$ of $100 \mu \mathrm{M}$ valinomycin solution was added to dissipate the membrane potential. The potential is assessed as the difference between the fluorescence before and after addition of the valinomycin. 


\section{Bibliography}

Abe, Y., Shodai, T., Muto, T., Mihara, K., Torii, H., Nishikawa, S., Endo, T. et al. (2000). Structural basis of presequence recognition by the mitochondrial protein import receptor Tom20. Cell, 100(5):551-560.

von Ahsen, O., Voos, W., Henninger, H. and Pfanner, N. (1995). The mitochondrial protein import machinery. Role of ATP in dissociation of the Hsp70.Mim44 complex. J Biol Chem, 270(50):29848-29853.

Ahting, U., Thieffry, M., Hegerl, R., Neupert, W. and Nussberger, S. (2001). Tom40, the pore-forming component of the protein-conducting TOM channel in the outer membrane of mitochondria. J Cell Biol, 153(6):1151-1160.

Ahting, U., Thun, C., Hegerl, R., Typke, D., Nargang, F.E., Neupert, W. and Nussberger, S. (1999). The TOM core complex: the general protein import pore of the outer membrane of mitochondria. J Cell Biol, 147(5):959-968.

Albrecht, R., Rehling, P., Chacinska, A., Brix, J., Cadamuro, S., Volkmer, R., Guiard, B. et al. (2006). The Tim21 binding domain connects the preprotein translocases of both mitochondrial membranes. EMBO Rep, 7(12):1233-1238.

Alcock, F., Clements, A., Webb, C. and Lithgow, T. (2010). Evolution. Tinkering inside the organelle. Science, 327(5966):649-650.

Alconada, A., Kübrich, M., Moczko, M., Hönlinger, A. and Pfanner, N. (1995). The mitochondrial receptor complex: the small subunit Mom8b/Isp6 supports association of receptors with the general insertion pore and transfer of preproteins. Mol Cell Biol, 15(11):6196-6205.

Alder, N., Jensen, R.E. and Johnson, A. (2008a). Fluorescence mapping of mitochondrial TIM23 complex reveals a water-facing, substrate-interacting helix surface. Cell, 134(3):439-450.

Alder, N., Sutherland, J., Buhring, A., Jensen, R.E. and Johnson, A. (2008b). Quaternary structure of the mitochondrial TIM23 complex reveals dynamic association between Tim23p and other subunits. Mol Biol Cell, 19(1):159-170.

Allison, D. and Schatz, G. (1986). Artificial mitochondrial presequences. Proc Natl Acad Sci USA, 83(23):9011-9015. 
Andersson, S.G., Zomorodipour, A., Andersson, J.O., Sicheritz-Pontén, T., Alsmark, U.C., Podowski, R.M., Näslund, A.K. et al. (1998). The genome sequence of Rickettsia prowazekii and the origin of mitochondria. Nature, 396(6707):133-140.

Arnold, K., Bordoli, L., Kopp, J. and Schwede, T. (2006). The SWISS-MODEL workspace: a web-based environment for protein structure homology modelling. Bioinformatics, 22(2):195-201.

Bauer, M., Sirrenberg, C., Neupert, W. and Brunner, M. (1996). Role of Tim23 as voltage sensor and presequence receptor in protein import into mitochondria. Cell, 87(1):33-41.

Becker, T., Guiard, B., Thornton, N., Zufall, N., Stroud, D.A., Wiedemann, N. and Pfanner, N. (2010). Assembly of the mitochondrial protein import channel: role of Tom5 in two-stage interaction of Tom40 with the SAM complex. Mol Biol Cell, 21(18):31063113.

Becker, T., Horvath, S.E., Böttinger, L., Gebert, N., Daum, G. and Pfanner, N. (2013). Role of phosphatidylethanolamine in the biogenesis of mitochondrial outer membrane proteins. J Biol Chem, 288(23):16451-16459.

Becker, T., Pfannschmidt, S., Guiard, B., Stojanovski, D., Milenkovic, D., Kutik, S., Pfanner, N. et al. (2008). Biogenesis of the mitochondrial TOM complex: Mim1 promotes insertion and assembly of signal-anchored receptors. J Biol Chem, 283(1):120-127.

Becker, T., Wenz, L.S., Krüger, V., Lehmann, W., Müller, J.M., Goroncy, L., Zufall, N. et al. (2011). The mitochondrial import protein Mim1 promotes biogenesis of multispanning outer membrane proteins. J Cell Biol, 194(3):387-395.

Beilharz, T., Egan, B., Silver, P.A., Hofmann, K. and Lithgow, T. (2003). Bipartite signals mediate subcellular targeting of tail-anchored membrane proteins in Saccharomyces cerevisiae. J Biol Chem, 278(10):8219-8223.

Benz, R. (1994). Permeation of hydrophilic solutes through mitochondrial outer membranes: review on mitochondrial porins. Biochim Biophys Acta, 1197(2):167-196.

Berthold, J., Bauer, M.F., Schneider, H.C., Klaus, C., Dietmeier, K., Neupert, W. and Brunner, M. (1995). The MIM complex mediates preprotein translocation across the mitochondrial inner membrane and couples it to the mt-Hsp70/ATP driving system. Cell, 81(7):1085-1093.

Beverly, K.N., Sawaya, M.R., Schmid, E. and Koehler, C.M. (2008). The Tim8-Tim13 complex has multiple substrate binding sites and binds cooperatively to Tim23. J Mol Biol, 382(5):1144-1156. 
Bihlmaier, K., Mesecke, N., Terziyska, N., Bien, M., Hell, K. and Herrmann, J.M. (2007). The disulfide relay system of mitochondria is connected to the respiratory chain. $J$ Cell Biol, 179(3):389-395.

Blamowska, M., Neupert, W. and Hell, K. (2012). Biogenesis of the mitochondrial Hsp70 chaperone. J Cell Biol, 199(1):125-135.

Blamowska, M., Sichting, M., Mapa, K., Mokranjac, D., Neupert, W. and Hell, K. (2010). ATPase domain and interdomain linker play a key role in aggregation of mitochondrial Hsp70 chaperone Ssc1. J Biol Chem, 285(7):4423-4431.

Blobel, G. and Dobberstein, B. (1975). Transfer of proteins across membranes. I. Presence of proteolytically processed and unprocessed nascent immunoglobulin light chains on membrane-bound ribosomes of murine myeloma. J Cell Biol, 67(3):835-851.

Blom, J., Kübrich, M., Rassow, J., Voos, W., Dekker, P.J., Maarse, A.C., Meijer, M. et al. (1993). The essential yeast protein MIM44 (encoded by MPI1) is involved in an early step of preprotein translocation across the mitochondrial inner membrane. Mol Cell Biol, 13(12):7364-7371.

Bohnert, M., Rehling, P., Guiard, B., Herrmann, J.M., Pfanner, N. and van der Laan, M. (2010). Cooperation of stop-transfer and conservative sorting mechanisms in mitochondrial protein transport. Curr Biol, 20(13):1227-1232.

Bohnert, M., Wenz, L.S., Zerbes, R.M., Horvath, S.E., Stroud, D.A., von der Malsburg, K., Muller, J.M. et al. (2012). Role of mitochondrial inner membrane organizing system in protein biogenesis of the mitochondrial outer membrane. Mol Biol Cell, 23(20):39483956 .

Bömer, U., Meijer, M., Maarse, A.C., Hönlinger, A., Dekker, P.J., Pfanner, N. and Rassow, J. (1997). Multiple interactions of components mediating preprotein translocation across the inner mitochondrial membrane. EMBO J, 16(9):2205-2216.

Bonneaud, N., Ozier-Kalogeropoulos, O., Li, G.Y., Labouesse, M., Minvielle-Sebastia, L. and Lacroute, F. (1991). A family of low and high copy replicative, integrative and single-stranded S. cerevisiae/E. coli shuttle vectors. Yeast, 7(6):609-615.

Botelho, S.C., Osterberg, M., Reichert, A.S., Yamano, K., Björkholm, P., Endo, T., von Heijne, G. et al. (2011). TIM23-mediated insertion of transmembrane $\alpha$-helices into the mitochondrial inner membrane. EMBO J, 30(6):1003-1011.

Brachmann, C.B., Davies, A., Cost, G.J., Caputo, E., Li, J., Hieter, P. and Boeke, J.D. (1998). Designer deletion strains derived from Saccharomyces cerevisiae S288C: a useful set of strains and plasmids for PCR-mediated gene disruption and other applications. Yeast, 14(2):115-132. 
Brix, J., Dietmeier, K. and Pfanner, N. (1997). Differential recognition of preproteins by the purified cytosolic domains of the mitochondrial import receptors Tom20, Tom22, and Tom70. J Biol Chem, 272(33):20730-20735.

Brix, J., Rüdiger, S., Bukau, B., Schneider-Mergener, J. and Pfanner, N. (1999). Distribution of binding sequences for the mitochondrial import receptors Tom20, Tom22, and Tom70 in a presequence-carrying preprotein and a non-cleavable preprotein. $J$ Biol Chem, 274(23):16522-16530.

Burri, L., Vascotto, K., Fredersdorf, S., Tiedt, R., Hall, M.N. and Lithgow, T. (2004). Zim17, a novel zinc finger protein essential for protein import into mitochondria. $J$ Biol Chem, 279(48):50243-50249.

Campello, S. and Scorrano, L. (2010). Mitochondrial shape changes: orchestrating cell pathophysiology. EMBO Rep, 11(9):678-684.

Chacinska, A., Koehler, C.M., Milenkovic, D., Lithgow, T. and Pfanner, N. (2009). Importing mitochondrial proteins: machineries and mechanisms. Cell, 138(4):628-644.

Chacinska, A., van der Laan, M., Mehnert, C., Guiard, B., Mick, D.U., Hutu, D.P., Truscott, K. et al. (2010). Distinct forms of mitochondrial TOM-TIM supercomplexes define signal-dependent states of preprotein sorting. Mol Cell Biol, 30(1):307-318.

Chacinska, A., Lind, M., Frazier, A.E., Dudek, J., Meisinger, C., Geissler, A., Sickmann, A. et al. (2005). Mitochondrial Presequence Translocase: Switching between TOM Tethering and Motor Recruitment Involves Tim21 and Tim17. Cell, 120(6):817-829.

Chacinska, A., Rehling, P., Guiard, B., Frazier, A.E., Schulze-Specking, A., Pfanner, N., Voos, W. et al. (2003). Mitochondrial translocation contact sites: separation of dynamic and stabilizing elements in formation of a TOM-TIM-preprotein supercomplex. EMBO J, 22(20):5370-5381.

Clapham, D.E. (2007). Calcium signaling. Cell, 131(6):1047-1058.

Claypool, S.M. (2009). Cardiolipin, a critical determinant of mitochondrial carrier protein assembly and function. Biochim Biophys Acta, 1788(10):2059-2068.

Cox, J.S., Chapman, R.E. and Walter, P. (1997). The unfolded protein response coordinates the production of endoplasmic reticulum protein and endoplasmic reticulum membrane. Mol Biol Cell, 8(9):1805-1814.

de la Cruz, L., Bajaj, R., Becker, S. and Zweckstetter, M. (2010). The intermembrane space domain of Tim23 is intrinsically disordered with a distinct binding region for presequences. Protein Sci, 19(11):2045-2054. 
Curran, B.P.G. and Bugeja, V. (2006). Basic investigations in Saccharomyces cerevisiae. Methods Mol. Biol., 313:1-13.

Daley, D.O., Adams, K.L., Clifton, R., Qualmann, S., Millar, A.H., Palmer, J.D., Pratje, E. et al. (2002). Gene transfer from mitochondrion to nucleus: novel mechanisms for gene activation from Cox2. Plant J, 30(1):11-21.

Davis, A.J., Alder, N.N., Jensen, R.E. and Johnson, A.E. (2007). The Tim9p/10p and Tim8p/13p complexes bind to specific sites on Tim23p during mitochondrial protein import. Mol Biol Cell, 18(2):475-486.

Dekker, P.J., Keil, P., Rassow, J., Maarse, A.C., Pfanner, N. and Meijer, M. (1993). Identification of MIM23, a putative component of the protein import machinery of the mitochondrial inner membrane. FEBS Lett, 330(1):66-70.

Dekker, P.J., Martin, F., Maarse, A.C., Bömer, U., Müller, H., Guiard, B., Meijer, M. et al. (1997). The Tim core complex defines the number of mitochondrial translocation contact sites and can hold arrested preproteins in the absence of matrix Hsp70-Tim44. EMBO J, 16(17):5408-5419.

Dietmeier, K., Hönlinger, A., Bömer, U., Dekker, P.J., Eckerskorn, C., Lottspeich, F., Kübrich, M. et al. (1997). Tom5 functionally links mitochondrial preprotein receptors to the general import pore. Nature, 388(6638):195-200.

Dimmer, K.S., Papic, D., Schumann, B., Sperl, D., Krumpe, K., Walther, D.M. and Rapaport, D. (2012). A crucial role for Mim2 in the biogenesis of mitochondrial outer membrane proteins. J Cell Sci, 125(Pt 14):3464-3473.

Dimova, K., Kawabe, H., Betz, A., Brose, N. and Jahn, O. (2006). Characterization of the Munc13-calmodulin interaction by photoaffinity labeling. Biochim Biophys Acta, 1763(11):1256-1265.

Donzeau, M., Káldi, K., Adam, A., Paschen, S., Wanner, G., Guiard, B., Bauer, M.F. et al. (2000). Tim23 links the inner and outer mitochondrial membranes. Cell, 101(4):401-412.

Dormán, G. and Prestwich, G. (2000). Using photolabile ligands in drug discovery and development. Trends Biotechnol, 18(2):64-77.

D'Silva, P., Schilke, B., Hayashi, M. and Craig, E.A. (2008). Interaction of the J-protein heterodimer Pam18/Pam16 of the mitochondrial import motor with the translocon of the inner membrane. Mol Biol Cell, 19(1):424-432. 
D'Silva, P., Schilke, B., Walter, W., Andrew, A. and Craig, E.A. (2003). J protein cochaperone of the mitochondrial inner membrane required for protein import into the mitochondrial matrix. Proc Natl Acad Sci USA, 100(24):13839-13844.

Dudek, J., Rehling, P. and van der Laan, M. (2013). Mitochondrial protein import: common principles and physiological networks. Biochim Biophys Acta, 1833(2):274285.

de Duve, C. (2007). The origin of eukaryotes: a reappraisal. Nat Rev Genet, 8(5):395-403.

Eilers, M. and Schatz, G. (1986). Binding of a specific ligand inhibits import of a purified precursor protein into mitochondria. Nature, 322(6076):228-232.

Eliyahu, E., Pnueli, L., Melamed, D., Scherrer, T., Gerber, A.P., Pines, O., Rapaport, D. et al. (2009). Tom20 Mediates Localization of mRNAs to Mitochondria in a TranslationDependent Manner. Mol Cell Biol, 30(1):284-294.

Endo, T., Yamano, K. and Kawano, S. (2011). Structural insight into the mitochondrial protein import system. BBA - Biomembranes, 1808(3):955-970.

Erdmann, R., Wiebel, F.F., Flessau, A., Rytka, J., Beyer, A., Fröhlich, K.U. and Kunau, W.H. (1991). PAS1, a yeast gene required for peroxisome biogenesis, encodes a member of a novel family of putative ATPases. Cell, 64(3):499-510.

Esaki, M., Kanamori, T., ichi Nishikawa, S., Shin, I., Schultz, P. and Endo, T. (2003). Tom40 protein import channel binds to non-native proteins and prevents their aggregation. Nat Struct Biol, 10(12):988-994.

Esaki, M., Shimizu, H., Ono, T., Yamamoto, H., Kanamori, T., Nishikawa, S.I. and Endo, T. (2004). Mitochondrial protein import. Requirement of presequence elements and tom components for precursor binding to the TOM complex. J Biol Chem, 279(44):4570145707.

Flores-Pérez, Ú. and Jarvis, P. (2013). Molecular chaperone involvement in chloroplast protein import. BBA - Molecular Cell Research, 1833(2):332-340.

Fölsch, H., Guiard, B., Neupert, W. and Stuart, R.A. (1996). Internal targeting signal of the BCS1 protein: a novel mechanism of import into mitochondria. EMBO J, 15(3):479-487.

Fraga, H., Papaleo, E., Vega, S., Velazquez-Campoy, A. and Ventura, S. (2013). Zinc induced folding is essential for TIM15 activity as an mtHsp70 chaperone. BBA - General Subjects, 1830(1):2139-2149. 
Frazier, A.E., Chacinska, A., Truscott, K., Guiard, B., Pfanner, N. and Rehling, P. (2003). Mitochondria use different mechanisms for transport of multispanning membrane proteins through the intermembrane space. Mol Cell Biol, 23(21):7818-7828.

Frazier, A.E., Dudek, J., Guiard, B., Voos, W., Li, Y., Lind, M., Meisinger, C. et al. (2004). Pam16 has an essential role in the mitochondrial protein import motor. Nat Struct Mol Biol, 11(3):226-233.

Frazier, A.E., Mick, D.U., Warscheid, B., Stoepel, N., Meyer, H.E., Ryan, M.T., Guiard, B. et al. (2006). Mdm38 interacts with ribosomes and is a component of the mitochondrial protein export machinery. J Cell Biol, 172(4):553-564.

Gaikwad, A. and Cumsky, M. (1994). The use of chemical cross-linking to identify proteins that interact with a mitochondrial presequence. J Biol Chem, 269(9):6437-6443.

Gallagher, S., Winston, S.E., Fuller, S.A. and Hurrell, J.G.R. (2004). Immunoblotting and immunodetection. Curr Protoc Mol Biol, Chapter 10:Unit 10.8.

Gambill, B.D., Voos, W., Kang, P.J., Miao, B., Langer, T., Craig, E.A. and Pfanner, N. (1993). A dual role for mitochondrial heat shock protein 70 in membrane translocation of preproteins. J Cell Biol, 123(1):109-117.

Gärtner, F., Voos, W., Querol, A., Miller, B.R., Craig, E.A., Cumsky, M.G. and Pfanner, N. (1995). Mitochondrial import of subunit Va of cytochrome c oxidase characterized with yeast mutants. J Biol Chem, 270(8):3788-3795.

Gaume, B., Klaus, C., Ungermann, C., Guiard, B., Neupert, W. and Brunner, M. (1998). Unfolding of preproteins upon import into mitochondria. EMBO J, 17(22):6497-6507.

Gebert, M., Schrempp, S.G., Mehnert, C.S., Heißwolf, A.K., Oeljeklaus, S., Ieva, R., Bohnert, M. et al. (2012). Mgr2 promotes coupling of the mitochondrial presequence translocase to partner complexes. J Cell Biol.

Gebert, N., Chacinska, A., Wagner, K., Guiard, B., Koehler, C.M., Rehling, P., Pfanner, N. et al. (2008). Assembly of the three small Tim proteins precedes docking to the mitochondrial carrier translocase. EMBO Rep, 9(6):548-554.

Gebert, N., Gebert, M., Oeljeklaus, S., Von Der Malsburg, K., Stroud, D.A., Kulawiak, B., Wirth, C. et al. (2011). Dual function of Sdh3 in the respiratory chain and TIM22 protein translocase of the mitochondrial inner membrane. Mol Cell, 44(5):811-818.

Geissler, A., Chacinska, A., Truscott, K., Wiedemann, N., Brandner, K., Sickmann, A., Meyer, H.E. et al. (2002). The mitochondrial presequence translocase: an essential role of Tim50 in directing preproteins to the import channel. Cell, 111(4):507-518. 
Geissler, A., Krimmer, T., Bömer, U., Guiard, B., Rassow, J. and Pfanner, N. (2000). Membrane potential-driven protein import into mitochondria. The sorting sequence of cytochrome $\mathrm{b}(2)$ modulates the deltapsi-dependence of translocation of the matrixtargeting sequence. Mol Biol Cell, 11(11):3977-3991.

Geissler, A., Rassow, J., Pfanner, N. and Voos, W. (2001). Mitochondrial import driving forces: enhanced trapping by matrix Hsp70 stimulates translocation and reduces the membrane potential dependence of loosely folded preproteins. Mol Cell Biol, 21(20):7097-7104.

Gevorkyan-Airapetov, L., Zohary, K., Popov-Celeketić, D., Mapa, K., Hell, K., Neupert, W., Azem, A. et al. (2009). Interaction of Tim23 with Tim50 Is essential for protein translocation by the mitochondrial TIM23 complex. J Biol Chem, 284(8):4865-4872.

Gietz, R.D. and Woods, R.A. (2002). Transformation of yeast by lithium acetate/singlestranded carrier DNA/polyethylene glycol method. Meth. Enzymol., 350:87-96.

van der Giezen, M. and Tovar, J. (2005). Degenerate mitochondria. EMBO Rep, 6(6):525530 .

Gillespie, L.L. (1987). Identification of an outer mitochondrial membrane protein that interacts with a synthetic signal peptide. J Biol Chem, 262(17):7939-7942.

Gillespie, L.L., Argan, C., Taneja, A.T., Hodges, R.S., Freeman, K.B. and Shore, G.C. (1985). A synthetic signal peptide blocks import of precursor proteins destined for the mitochondrial inner membrane or matrix. J Biol Chem, 260(30):16045-16048.

Glaser, S. and Cumsky, M. (1990). A synthetic presequence reversibly inhibits protein import into yeast mitochondria. J Biol Chem, 265(15):8808-8816.

Glaser, S.M., Miller, B.R. and Cumsky, M.G. (1990). Removal of a hydrophobic domain within the mature portion of a mitochondrial inner membrane protein causes its mislocalization to the matrix. Mol Cell Biol, 10(5):1873-1881.

Glick, B., Brandt, A., Cunningham, K., Müller, S., Hallberg, R. and Schatz, G. (1992). Cytochromes $\mathrm{c} 1$ and $\mathrm{b} 2$ are sorted to the intermembrane space of yeast mitochondria by a stop-transfer mechanism. Cell, 69(5):809-822.

Glick, B.S. (1995). Can Hsp70 proteins act as force-generating motors? Cell, 80(1):11-14.

Glick, B.S., Wachter, C., Reid, G.A. and Schatz, G. (1993). Import of cytochrome b2 to the mitochondrial intermembrane space: the tightly folded heme-binding domain makes import dependent upon matrix ATP. Protein Sci, 2(11):1901-1917. 
Green, D.R. and Reed, J.C. (1998). Mitochondria and apoptosis. Science, 281(5381):13091312 .

Green-Willms, N.S., Butler, C.A., Dunstan, H.M. and Fox, T.D. (2001). Pet111p, an inner membrane-bound translational activator that limits expression of the Saccharomyces cerevisiae mitochondrial gene COX2. J Biol Chem, 276(9):6392-6397.

Greene, M., Maskos, K. and Landry, S. (1998). Role of the J-domain in the cooperation of Hsp40 with Hsp70. Proc Natl Acad Sci USA, 95(11):6108-6113.

Gruhler, A., Arnold, I., Seytter, T., Guiard, B., Schwarz, E., Neupert, W. and Stuart, R.A. (1997). N-terminal hydrophobic sorting signals of preproteins confer mitochondrial hsp70 independence for import into mitochondria. J Biol Chem, 272(28):17410-17415.

Hahne, K., Haucke, V., Ramage, L. and Schatz, G. (1994). Incomplete arrest in the outer membrane sorts NADH-cytochrome b5 reductase to two different submitochondrial compartments. Cell, 79(5):829-839.

Haltia, T. and Freire, E. (1995). Forces and factors that contribute to the structural stability of membrane proteins. Biochim Biophys Acta, 1241(2):295-322.

Hammen, P. and Weiner, H. (1998). Mitochondrial leader sequences: structural similarities and sequence differences. J Exp Zool, 282(1-2):280-283.

Hanahan, D. (1983). Studies on transformation of Escherichia coli with plasmids. J Mol Biol, 166(4):557-580.

Handa, N., Kishishita, S., Morita, S., Akasaka, R., Jin, Z., Chrzas, J., Chen, L. et al. (2007). Structure of the human Tim44 C-terminal domain in complex with pentaethylene glycol: ligand-bound form. Acta Crystallogr. D Biol. Crystallogr., 63(Pt 12):12251234 .

Harner, M., Neupert, W. and Deponte, M. (2011). Lateral release of proteins from the TOM complex into the outer membrane of mitochondria. EMBO J, 30(16):3232-3241.

Hartl, F.U. and Hayer-Hartl, M. (2002). Molecular chaperones in the cytosol: from nascent chain to folded protein. Science, 295(5561):1852-1858.

Hartl, F.U., Ostermann, J., Guiard, B. and Neupert, W. (1987). Successive translocation into and out of the mitochondrial matrix: targeting of proteins to the intermembrane space by a bipartite signal peptide. Cell, 51(6):1027-1037.

Hawlitschek, G., Schneider, H., Schmidt, B., Tropschug, M., Hartl, F.U. and Neupert, W. (1988). Mitochondrial protein import: identification of processing peptidase and of PEP, a processing enhancing protein. Cell, 53(5):795-806. 
von Heijne, G. (1986). Mitochondrial targeting sequences may form amphiphilic helices. EMBO J, 5(6):1335-1342.

Hell, K., Herrmann, J.M., Pratje, E., Neupert, W. and Stuart, R.A. (1998). Oxa1p, an essential component of the N-tail protein export machinery in mitochondria. Proc Natl Acad Sci USA, 95(5):2250-2255.

Heo, J.M., Taylor, E.B., Dephoure, N., Brodsky, J.L., Gygi, S.P. and Rutter, J. (2010). A Stress-Responsive System for Mitochondrial Protein Degradation. Mol Cell, 40(3):465480 .

Herrmann, J.M., Neupert, W. and Stuart, R.A. (1997). Insertion into the mitochondrial inner membrane of a polytopic protein, the nuclear-encoded Oxa1p. EMBO J, 16(9):2217-2226.

Herrmann, J.M. and Riemer, J. (2012). Mitochondrial disulfide relay: redox-regulated protein import into the intermembrane space. J Biol Chem, 287(7):4426-4433.

Hill, K., Model, K., Ryan, M., Dietmeier, K., Martin, F., Wagner, R. and Pfanner, N. (1998). Tom40 forms the hydrophilic channel of the mitochondrial import pore for preproteins [see comment]. Nature, 395(6701):516-521.

Hines, V., Brandt, A., Griffiths, G., Horstmann, H., Brütsch, H. and Schatz, G. (1990). Protein import into yeast mitochondria is accelerated by the outer membrane protein MAS70. EMBO J, 9(10):3191-3200.

Hines, V. and Schatz, G. (1993). Precursor binding to yeast mitochondria. A general role for the outer membrane protein Mas70p. J Biol Chem, 268(1):449-454.

Hönlinger, A., Bömer, U., Alconada, A., Eckerskorn, C., Lottspeich, F., Dietmeier, K. and Pfanner, N. (1996). Tom7 modulates the dynamics of the mitochondrial outer membrane translocase and plays a pathway-related role in protein import. EMBO J, 15(9):2125-2137.

Horst, M., Oppliger, W., Feifel, B., Schatz, G. and Glick, B. (1996). The mitochondrial protein import motor: dissociation of mitochondrial hsp70 from its membrane anchor requires ATP binding rather than ATP hydrolysis. Protein Sci, 5(4):759-767.

Hulett, J.M., Walsh, P. and Lithgow, T. (2007). Domain stealing by receptors in a protein transport complex. Mol Biol Evol, 24(9):1909-1911.

Hutu, D.P., Guiard, B., Chacinska, A., Becker, D., Pfanner, N., Rehling, P. and van der Laan, M. (2008). Mitochondrial protein import motor: differential role of Tim44 in the recruitment of Pam17 and J-complex to the presequence translocase. Mol Biol Cell, 19(6):2642-2649. 
Iosefson, O., Levy, R., Marom, M., Slutsky-Leiderman, O. and Azem, A. (2007). The Pam18/Tim14-Pam16/Tim16 complex of the mitochondrial translocation motor: the formation of a stable complex from marginally stable proteins. Protein Sci, 16(2):316322.

Jahn, O., Eckart, K., Brauns, O., Tezval, H. and Spiess, J. (2002). The binding protein of corticotropin-releasing factor: ligand-binding site and subunit structure. Proc Natl Acad Sci USA, 99(19):12055-12060.

Jahn, O., Eckart, K., Tezval, H. and Spiess, J. (2004). Characterization of peptide-protein interactions using photoaffinity labeling and LC/MS. Anal Bioanal Chem, 378(4):10311036.

Janke, C., Magiera, M., Rathfelder, N., Taxis, C., Reber, S., Maekawa, H., MorenoBorchart, A. et al. (2004). A versatile toolbox for PCR-based tagging of yeast genes: new fluorescent proteins, more markers and promoter substitution cassettes. Yeast, 21(11):947-962.

Jia, L., Dienhart, M., Schramp, M., McCauley, M., Hell, K. and Stuart, R.A. (2003). Yeast Oxa1 interacts with mitochondrial ribosomes: the importance of the C-terminal region of Oxa1. EMBO J, 22(24):6438-6447.

Jiang, J., Maes, E., Taylor, A., Wang, L., Hinck, A., Lafer, E. and Sousa, R. (2007). Structural basis of J cochaperone binding and regulation of Hsp70. Mol Cell, 28(3):422433.

Jiang, J., Prasad, K., Lafer, E. and Sousa, R. (2005). Structural basis of interdomain communication in the Hsc70 chaperone. Mol Cell, 20(4):513-524.

Josyula, R., Jin, Z., Fu, Z. and Sha, B. (2006). Crystal structure of yeast mitochondrial peripheral membrane protein Tim44p C-terminal domain. J Mol Biol, 359(3):798-804.

Junge, H.J., Rhee, J.S., Jahn, O., Varoqueaux, F., Spiess, J., Waxham, M.N., Rosenmund, C. et al. (2004). Calmodulin and Munc13 form a Ca2+ sensor/effector complex that controls short-term synaptic plasticity. Cell, 118(3):389-401.

Kanaji, S., Iwahashi, J., Kida, Y., Sakaguchi, M. and Mihara, K. (2000). Characterization of the signal that directs Tom20 to the mitochondrial outer membrane. J Cell Biol, 151(2):277-288.

Kanamori, T., Nishikawa, S., Nakai, M., Shin, I., Schultz, P. and Endo, T. (1999). Uncoupling of transfer of the presequence and unfolding of the mature domain in precursor translocation across the mitochondrial outer membrane. Proc Natl Acad Sci USA, 96(7):3634-3639. 
Kanamori, T., Nishikawa, S., Shin, I., Schultz, P. and Endo, T. (1997). Probing the environment along the protein import pathways in yeast mitochondria by site-specific photocrosslinking. Proc Natl Acad Sci USA, 94(2):485-490.

Kang, P.J., Ostermann, J., Shilling, J., Neupert, W., Craig, E.A. and Pfanner, N. (1990). Requirement for hsp70 in the mitochondrial matrix for translocation and folding of precursor proteins. Nature, 348(6297):137-143.

Kemper, C., Habib, S.J., Engl, G., Heckmeyer, P., Dimmer, K.S. and Rapaport, D. (2008). Integration of tail-anchored proteins into the mitochondrial outer membrane does not require any known import components. J Cell Sci, 121(Pt 12):1990-1998.

Kerscher, O., Holder, J., Srinivasan, M., Leung, R.S. and Jensen, R.E. (1997). The Tim54p-Tim22p complex mediates insertion of proteins into the mitochondrial inner membrane. J Cell Biol, 139(7):1663-1675.

Kerscher, O., Sepuri, N.B. and Jensen, R.E. (2000). Tim18p is a new component of the Tim54p-Tim22p translocon in the mitochondrial inner membrane. Mol Biol Cell, 11(1):103-116.

Koehler, C.M., Murphy, M.P., Bally, N.A., Leuenberger, D., Oppliger, W., Dolfini, L., Junne, T. et al. (2000). Tim18p, a new subunit of the TIM22 complex that mediates insertion of imported proteins into the yeast mitochondrial inner membrane. Mol Cell Biol, 20(4):1187-1193.

Koll, H., Guiard, B., Rassow, J., Ostermann, J., Horwich, A.L., Neupert, W. and Hartl, F.U. (1992). Antifolding activity of hsp60 couples protein import into the mitochondrial matrix with export to the intermembrane space. Cell, 68(6):1163-1175.

Komiya, T., Rospert, S., Koehler, C.M., Looser, R., Schatz, G. and Mihara, K. (1998). Interaction of mitochondrial targeting signals with acidic receptor domains along the protein import pathway: evidence for the 'acid chain' hypothesis. EMBO J, 17(14):38863898.

Komiya, T., Rospert, S., Schatz, G. and Mihara, K. (1997). Binding of mitochondrial precursor proteins to the cytoplasmic domains of the import receptors Tom70 and Tom20 is determined by cytoplasmic chaperones. EMBO J, 16(14):4267-4275.

Komiya, T., Sakaguchi, M. and Mihara, K. (1996). Cytoplasmic chaperones determine the targeting pathway of precursor proteins to mitochondria. EMBO J, 15(2):399-407.

Komuro, Y., Miyashita, N., Mori, T., Muneyuki, E., Saitoh, T., Kohda, D. and Sugita, Y. (2013). Energetics of the presequence-binding poses in mitochondrial protein import through Tom20. J Phys Chem B, 117(10):2864-2871. 
Kovermann, P., Truscott, K.N., Guiard, B., Rehling, P., Sepuri, N.B., Müller, H., Jensen, R.E. et al. (2002). Tim22, the essential core of the mitochondrial protein insertion complex, forms a voltage-activated and signal-gated channel. Mol Cell, 9(2):363-373.

Kozany, C., Mokranjac, D., Sichting, M., Neupert, W. and Hell, K. (2004). The J domainrelated cochaperone Tim16 is a constituent of the mitochondrial TIM23 preprotein translocase. Nat Struct Mol Biol, 11(3):234-241.

Krimmer, T., Rapaport, D., Ryan, M.T., Meisinger, C., Kassenbrock, C.K., BlachlyDyson, E., Forte, M. et al. (2001). Biogenesis of porin of the outer mitochondrial membrane involves an import pathway via receptors and the general import pore of the TOM complex. J Cell Biol, 152(2):289-300.

Krimmer, T., Rassow, J., Kunau, W., Voos, W. and Pfanner, N. (2000). Mitochondrial protein import motor: the ATPase domain of matrix Hsp70 is crucial for binding to Tim44, while the peptide binding domain and the carboxy-terminal segment play a stimulatory role. Mol Cell Biol, 20(16):5879-5887.

Kronidou, N.G., Oppliger, W., Bolliger, L., Hannavy, K., Glick, B.S., Schatz, G. and Horst, M. (1994). Dynamic interaction between Isp45 and mitochondrial hsp70 in the protein import system of the yeast mitochondrial inner membrane. Proc Natl Acad Sci USA, 91(26):12818-12822.

Krumpe, K., Frumkin, I., Herzig, Y., Rimon, N., Ozbalci, C., Brugger, B., Rapaport, D. et al. (2012). Ergosterol content specifies targeting of tail-anchored proteins to mitochondrial outer membranes. Mol Biol Cell, 23(20):3927-3935.

Kübrich, M., Keil, P., Rassow, J., Dekker, P.J., Blom, J., Meijer, M. and Pfanner, N. (1994). The polytopic mitochondrial inner membrane proteins MIM17 and MIM23 operate at the same preprotein import site. FEBS Lett, 349(2):222-228.

Künkele, K.P., Heins, S., Dembowski, M., Nargang, F.E., Benz, R., Thieffry, M., Walz, J. et al. (1998a). The preprotein translocation channel of the outer membrane of mitochondria. Cell, 93(6):1009-1019.

Künkele, K.P., Juin, P., Pompa, C., Nargang, F.E., Henry, J.P., Neupert, W., Lill, R. et al. (1998b). The isolated complex of the translocase of the outer membrane of mitochondria. Characterization of the cation-selective and voltage-gated preproteinconducting pore. J Biol Chem, 273(47):31032-31039.

Kutik, S., Stojanovski, D., Becker, L., Becker, T., Meinecke, M., Krüger, V., Prinz, C. et al. (2008). Dissecting membrane insertion of mitochondrial beta-barrel proteins. Cell, 132(6):1011-1024. 
van der Laan, M., Bohnert, M., Wiedemann, N. and Pfanner, N. (2012). Role of MINOS in mitochondrial membrane architecture and biogenesis. Trends Cell Biol, 22(4):185-192.

van der Laan, M., Chacinska, A., Lind, M., Perschil, I., Sickmann, A., Meyer, H.E., Guiard, B. et al. (2005). Pam17 is required for architecture and translocation activity of the mitochondrial protein import motor. Mol Cell Biol, 25(17):7449-7458.

van der Laan, M., Hutu, D.P. and Rehling, P. (2010). On the mechanism of preprotein import by the mitochondrial presequence translocase. Biochim Biophys Acta, 1803(6):732-739.

van der Laan, M., Meinecke, M., Dudek, J., Hutu, D.P., Lind, M., Perschil, I., Guiard, B. et al. (2007). Motor-free mitochondrial presequence translocase drives membrane integration of preproteins. Nat Cell Biol, 9(10):1152-1159.

van der Laan, M., Wiedemann, N., Mick, D.U., Guiard, B., Rehling, P. and Pfanner, N. (2006). A Role for Tim21 in Membrane-Potential-Dependent Preprotein Sorting in Mitochondria. Current Biology, 16(22):2271-2276.

Laemmli, U.K. (1970). Cleavage of structural proteins during the assembly of the head of bacteriophage T4. Nature, 227(5259):680-685.

Laloraya, S., Gambill, B.D. and Craig, E.A. (1994). A role for a eukaryotic GrpE-related protein, Mge1p, in protein translocation. Proc Natl Acad Sci USA, 91(14):6481-6485.

Lane, N. and Martin, W. (2010). The energetics of genome complexity. Nature, 467(7318):929-934.

Langer, T., Lu, C., Echols, H., Flanagan, J., Hayer, M. and Hartl, F.U. (1992). Successive action of DnaK, DnaJ and GroEL along the pathway of chaperone-mediated protein folding. Nature, 356(6371):683-689.

Lee, C.M. (1999). The DNA Helicase, Hmi1p, Is Transported into Mitochondria by a C-terminal Cleavable Targeting Signal. J Biol Chem, 274(30):20937-20942.

Li, Y., Dudek, J., Guiard, B., Pfanner, N., Rehling, P. and Voos, W. (2004). The presequence translocase-associated protein import motor of mitochondria. Pam16 functions in an antagonistic manner to Pam18. J Biol Chem, 279(36):38047-38054.

Liberek, K., Marszalek, J., Ang, D., Georgopoulos, C. and Zylicz, M. (1991). Escherichia coli DnaJ and GrpE heat shock proteins jointly stimulate ATPase activity of DnaK. Proc Natl Acad Sci USA, 88(7):2874-2878. 
Lill, R., Hoffmann, B., Molik, S., Pierik, A.J., Rietzschel, N., Stehling, O., Uzarska, M.A. et al. (2012). The role of mitochondria in cellular iron-sulfur protein biogenesis and iron metabolism. Biochimica et Biophysica Acta (BBA) - Molecular Cell Research, 1823(9):1491-1508.

Lithgow, T., Junne, T., Suda, K., Gratzer, S. and Schatz, G. (1994a). The mitochondrial outer membrane protein Mas22p is essential for protein import and viability of yeast. Proc Natl Acad Sci USA, 91(25):11973-11977.

Lithgow, T., Junne, T., Wachter, C. and Schatz, G. (1994b). Yeast mitochondria lacking the two import receptors Mas20p and Mas70p can efficiently and specifically import precursor proteins. J Biol Chem, 269(21):15325-15330.

Lithgow, T. and Schneider, A. (2010). Evolution of macromolecular import pathways in mitochondria, hydrogenosomes and mitosomes. Philosophical Transactions of the Royal Society B: Biological Sciences, 365(1541):799-817.

Liu, Q., D’Silva, P., Walter, W., Marszalek, J. and Craig, E.A. (2003). Regulated cycling of mitochondrial Hsp70 at the protein import channel. Science, 300(5616):139-141.

Lohret, T.A., Jensen, R.E. and Kinnally, K.W. (1997). Tim23, a protein import component of the mitochondrial inner membrane, is required for normal activity of the multiple conductance channel, MCC. J Cell Biol, 137(2):377-386.

van Loon, A.P., Brändli, A.W. and Schatz, G. (1986). The presequences of two imported mitochondrial proteins contain information for intracellular and intramitochondrial sorting. Cell, 44(5):801-812.

Lytovchenko, O., Melin, J., Schulz, C., Kilisch, M., Hutu, D.P. and Rehling, P. (2013). Signal recognition initiates reorganization of the presequence translocase during protein import. EMBO J, 32:886-898.

Maarse, A.C., Blom, J., Keil, P., Pfanner, N. and Meijer, M. (1994). Identification of the essential yeast protein MIM17, an integral mitochondrial inner membrane protein involved in protein import. FEBS Lett, 349(2):215-221.

Maccecchini, M.L., Rudin, Y. and Schatz, G. (1979). Transport of proteins across the mitochondrial outer membrane. A precursor form of the cytoplasmically made intermembrane enzyme cytochrome c peroxidase. J Biol Chem, 254(16):7468-7471.

Malhotra, K., Sathappa, M., Landin, J.S., Johnson, A.E. and Alder, N.N. (2013). Structural changes in the mitochondrial Tim23 channel are coupled to the proton-motive force. Nat Struct Mol Biol, 20(8):965-972. 
Mapa, K., Sikor, M., Kudryavtsev, V., Waegemann, K., Kalinin, S., Seidel, C.A.M., Neupert, W. et al. (2010). The conformational dynamics of the mitochondrial Hsp70 chaperone. Mol Cell, 38(1):89-100.

Marc, P., Margeot, A., Devaux, F., Blugeon, C., Corral Debrinski, M. and Jacq, C. (2002). Genome-wide analysis of mRNAs targeted to yeast mitochondria. EMBO Rep, $3(2): 159-164$.

Marom, M., Dayan, D., Demishtein-Zohary, K., Mokranjac, D., Neupert, W. and Azem, A. (2011). Direct Interaction of Mitochondrial Targeting Presequences with Purified Components of the TIM23 Protein Complex. J Biol Chem, 286(51):43809-43815.

Marom, M., Safonov, R., Amram, S., Avneon, Y., Nachliel, E., Gutman, M., Zohary, K. et al. (2009). Interaction of the Tim44 C-terminal domain with negatively charged phospholipids. Biochemistry, 48(47):11185-11195.

Martin, J., Mahlke, K. and Pfanner, N. (1991). Role of an energized inner membrane in mitochondrial protein import. Delta psi drives the movement of presequences. J Biol Chem, 266(27):18051-18057.

Martinez-Caballero, S., Grigoriev, S., Herrmann, J.M., Campo, M. and Kinnally, K. (2007). Tim17p regulates the twin pore structure and voltage gating of the mitochondrial protein import complex TIM23. J Biol Chem, 282(6):3584-3593.

Maruyama, M., Miura, M. and Endo, T. (2012). The Tom40 assembly process probed using the attachment of different intramitochondrial sorting signals. Mol Biol Cell, 23(20):3936-3947.

Matlack, K., Misselwitz, B., Plath, K. and Rapoport, T.A. (1999). BiP acts as a molecular ratchet during posttranslational transport of prepro-alpha factor across the ER membrane. Cell, 97(5):553-564.

Mayer, M. (2004). Timing the catch. Nat Struct Mol Biol, 11(1):6-8.

Meier, S., Neupert, W. and Herrmann, J.M. (2005a). Conserved N-terminal negative charges in the Tim17 subunit of the TIM23 translocase play a critical role in the import of preproteins into mitochondria. J Biol Chem, 280(9):7777-7785.

Meier, S., Neupert, W. and Herrmann, J.M. (2005b). Proline residues of transmembrane domains determine the sorting of inner membrane proteins in mitochondria. $J$ Cell Biol, 170(6):881-888.

Meinecke, M., Wagner, R., Kovermann, P., Guiard, B., Mick, D.U., Hutu, D.P., Voos, W. et al. (2006). Tim50 maintains the permeability barrier of the mitochondrial inner membrane. Science, 312(5779):1523-1526. 
Meineke, B., Engl, G., Kemper, C., Vasiljev-Neumeyer, A., Paulitschke, H. and Rapaport, D. (2008). The outer membrane form of the mitochondrial protein Mcr1 follows a TOMindependent membrane insertion pathway. FEBS Lett, 582(6):855-860.

Meisinger, C., Ryan, M., Hill, K., Model, K., Lim, J., Sickmann, A., Müller, H. et al. (2001). Protein import channel of the outer mitochondrial membrane: a highly stable Tom40-Tom22 core structure differentially interacts with preproteins, small tom proteins, and import receptors. Mol Cell Biol, 21(7):2337-2348.

Messerschmitt, M., Jakobs, S., Vogel, F., Fritz, S., Dimmer, K.S., Neupert, W. and Westermann, B. (2003). The inner membrane protein Mdm33 controls mitochondrial morphology in yeast. J Cell Biol, 160(4):553-564.

Miao, B., Davis, J. and Craig, E.A. (1997). Mge1 functions as a nucleotide release factor for Ssc1, a mitochondrial Hsp70 of Saccharomyces cerevisiae. J Mol Biol, 265(5):541552 .

Milisav, I., Moro, F., Neupert, W. and Brunner, M. (2001). Modular structure of the TIM23 preprotein translocase of mitochondria. J Biol Chem, 276(28):25856-25861.

Moczko, M., Bömer, U., Kübrich, M., Zufall, N., Hönlinger, A. and Pfanner, N. (1997). The intermembrane space domain of mitochondrial Tom22 functions as a trans binding site for preproteins with N-terminal targeting sequences. Mol Cell Biol, 17(11):65746584 .

Moczko, M., Dietmeier, K., Söllner, T., Segui, B., Steger, H.F., Neupert, W. and Pfanner, N. (1992). Identification of the mitochondrial receptor complex in Saccharomyces cerevisiae. FEBS Lett, 310(3):265-268.

Mokranjac, D., Berg, A., Adam, A., Neupert, W. and Hell, K. (2007). Association of the Tim14.Tim16 subcomplex with the TIM23 translocase is crucial for function of the mitochondrial protein import motor. J Biol Chem, 282(25):18037-18045.

Mokranjac, D., Bourenkov, G., Hell, K., Neupert, W. and Groll, M. (2006). Structure and function of Tim14 and Tim16, the J and J-like components of the mitochondrial protein import motor. EMBO J, 25(19):4675-4685.

Mokranjac, D. and Neupert, W. (2010). The many faces of the mitochondrial TIM23 complex. Biochim Biophys Acta, 1797(6-7):1045-1054.

Mokranjac, D., Paschen, S.A., Kozany, C., Prokisch, H., Hoppins, S.C., Nargang, F.E., Neupert, W. et al. (2003a). Tim50, a novel component of the TIM23 preprotein translocase of mitochondria. EMBO J,22(4):816-825. 
Mokranjac, D., Popov-Celeketić, D., Hell, K. and Neupert, W. (2005a). Role of Tim21 in mitochondrial translocation contact sites. J Biol Chem, 280(25):23437-23440.

Mokranjac, D., Sichting, M., Neupert, W. and Hell, K. (2003b). Tim14, a novel key component of the import motor of the TIM23 protein translocase of mitochondria. EMBO J, 22(19):4945-4956.

Mokranjac, D., Sichting, M., Popov-Celeketić, D., Berg, A., Hell, K. and Neupert, W. (2005b). The import motor of the yeast mitochondrial TIM23 preprotein translocase contains two different J proteins, Tim14 and Mdj2. J Biol Chem, 280(36):31608-31614.

Mokranjac, D., Sichting, M., Popov-Celeketić, D., Mapa, K., Gevorkyan-Airapetov, L., Zohary, K., Hell, K. et al. (2009). Role of Tim50 in the transfer of precursor proteins from the outer to the inner membrane of mitochondria. Mol Biol Cell, 20(5):1400-1407.

Moro, F., Sirrenberg, C., Schneider, H., Neupert, W. and Brunner, M. (1999). The TIM17.23 preprotein translocase of mitochondria: composition and function in protein transport into the matrix. EMBO J, 18(13):3667-3675.

Mumberg, D., Müller, R. and Funk, M. (1994). Regulatable promoters of Saccharomyces cerevisiae: comparison of transcriptional activity and their use for heterologous expression. Nucleic Acids Res, 22(25):5767-5768.

Muto, T., Obita, T., Abe, Y., Shodai, T., Endo, T. and Kohda, D. (2001). NMR identification of the Tom20 binding segment in mitochondrial presequences. J Mol Biol, 306(2):137-143.

Neuhoff, V., Arold, N., Taube, D. and Ehrhardt, W. (1988). Improved staining of proteins in polyacrylamide gels including isoelectric focusing gels with clear background at nanogram sensitivity using Coomassie Brilliant Blue G-250 and R-250. Electrophoresis, $9(6): 255-262$.

Neuhoff, V., Stamm, R. and Eibl, H. (1985). Clear Background and Highly Sensitive Protein Staining with Coomassie Blue Dyes in Polyacrylamide Gels - a Systematic Analysis. Electrophoresis, 6(9):427-448.

Neupert, W. (2012). SnapShot: Mitochondrial architecture. Cell, 149(3):722-722.e1.

Neupert, W. and Herrmann, J.M. (2007). Translocation of proteins into mitochondria. Annu Rev Biochem, 76:723-749.

Neupert, W. and Schatz, G. (1981). How proteins are transported into mitochondria. Trends in Biochem Sci, 6(0):1-4. 
Nguyen, T.T., Lewandowska, A., Choi, J.Y., Markgraf, D.F., Junker, M., Bilgin, M., Ejsing, C.S. et al. (2012). Gem1 and ERMES Do Not Directly Affect Phosphatidylserine Transport from ER to Mitochondria or Mitochondrial Inheritance. Traffic, 13(6):880890.

Nobrega, F.G., Nobrega, M.P. and Tzagoloff, A. (1992). BCS1, a novel gene required for the expression of functional Rieske iron-sulfur protein in Saccharomyces cerevisiae. EMBO J, 11(11):3821-3829.

Norrander, J. and Kempe, T. (1983). Construction of improved M13 vectors using oligodeoxynucleotide-directed mutagenesis. Gene, 26(1):101-106.

Obita, T., Muto, T., Endo, T. and Kohda, D. (2003). Peptide library approach with a disulfide tether to refine the Tom20 recognition motif in mitochondrial presequences. $J$ Mol Biol, 328(2):495-504.

Okamoto, K., Brinker, A., Paschen, S., Moarefi, I., Hayer-Hartl, M., Neupert, W. and Brunner, M. (2002). The protein import motor of mitochondria: a targeted molecular ratchet driving unfolding and translocation. EMBO J, 21(14):3659-3671.

Ono, H. and Tuboi, S. (1988). The cytosolic factor required for import of precursors of mitochondrial proteins into mitochondria. J Biol Chem, 263(7):3188-3193.

Ott, M., Prestele, M., Bauerschmitt, H., Funes, S., Bonnefoy, N. and Herrmann, J.M. (2006). Mba1, a membrane-associated ribosome receptor in mitochondria. EMBO J, 25(8):1603-1610.

Palade, G.E. (1964). THE ORGANIZATION OF LIVING MATTER. Proc Natl Acad Sci USA, 52:613-634.

Papic, D., Krumpe, K., Dukanovic, J., Dimmer, K.S. and Rapaport, D. (2011). Multispan mitochondrial outer membrane protein Ugo1 follows a unique Mim1-dependent import pathway. J Cell Biol, 194(3):397-405.

Park, E. and Rapoport, T.A. (2012). Mechanisms of Sec61/SecY-Mediated Protein Translocation Across Membranes. Annu Rev Biophys, 41:21-40.

Park, K., Botelho, S.C., Hong, J., Osterberg, M. and Kim, H. (2013). Dissecting stop transfer versus conservative sorting pathways for mitochondrial inner membrane proteins in vivo. J Biol Chem, 288(3):1521-1532.

Paumard, P., Vaillier, J., Coulary, B., Schaeffer, J., Soubannier, V., Mueller, D.M., Brèthes, D. et al. (2002). The ATP synthase is involved in generating mitochondrial cristae morphology. EMBO J, 21(3):221-230. 
Pfanner, N., Douglas, M.G., Endo, T., Hoogenraad, N.J., Jensen, R.E., Meijer, M., Neupert, W. et al. (1996). Uniform nomenclature for the protein transport machinery of the mitochondrial membranes. Trends in Biochem Sci, 21(2):51-52.

Pfanner, N. and Meijer, M. (1995). Protein sorting. Pulling in the proteins. Curr Biol, $5(2): 132-135$.

Pfanner, N., Tropschug, M. and Neupert, W. (1987). Mitochondrial protein import: nucleoside triphosphates are involved in conferring import-competence to precursors. Cell, 49(6):815-823.

Popov-Celeketić, D., Mapa, K., Neupert, W. and Mokranjac, D. (2008a). Active remodelling of the TIM23 complex during translocation of preproteins into mitochondria. EMBO J, 27(10):1469-1480.

Popov-Celeketić, D., Waegemann, K., Mapa, K., Neupert, W. and Mokranjac, D. (2011). Role of the import motor in insertion of transmembrane segments by the mitochondrial TIM23 complex. EMBO Rep, 12(6):542-548.

Popov-Celeketić, J., Waizenegger, T. and Rapaport, D. (2008b). Mim1 functions in an oligomeric form to facilitate the integration of Tom20 into the mitochondrial outer membrane. J Mol Biol, 376(3):671-680.

Pratje, E. and Guiard, B. (1986). One nuclear gene controls the removal of transient pre-sequences from two yeast proteins: one encoded by the nuclear the other by the mitochondrial genome. EMBO J, 5(6):1313-1317.

Prestwich, G.D., Dormán, G., Elliott, J.T., Marecak, D.M. and Chaudhary, A. (1997). Benzophenone photoprobes for phosphoinositides, peptides and drugs. Photochem. Photobiol., 65(2):222-234.

Qian, X., Gebert, M., Höpker, J., Yan, M., Li, J., Wiedemann, N., van der Laan, M. et al. (2011). Structural basis for the function of Tim50 in the mitochondrial presequence translocase. J Mol Biol, 411(3):513-519.

Qiu, J., Wenz, L.S., Zerbes, R.M., Oeljeklaus, S., Bohnert, M., Stroud, D.A., Wirth, C. et al. (2013). Coupling of Mitochondrial Import and Export Translocases by ReceptorMediated Supercomplex Formation. Cell, 154(3):596-608.

Rao, S., Gerbeth, C., Harbauer, A., Mikropoulou, D., Meisinger, C. and Schmidt, O. (2011). Signaling at the gate: phosphorylation of the mitochondrial protein import machinery. Cell Cycle, 10(13):2083-2090.

Rapaport, D. (2003). Finding the right organelle. Targeting signals in mitochondrial outer-membrane proteins. EMBO Rep, 4(10):948-952. 
Rapaport, D., Neupert, W. and Lill, R. (1997). Mitochondrial protein import. Tom40 plays a major role in targeting and translocation of preproteins by forming a specific binding site for the presequence. J Biol Chem, 272(30):18725-18731.

Rassow, J., Guiard, B., Wienhues, U., Herzog, V., Hartl, F.U. and Neupert, W. (1989). Translocation arrest by reversible folding of a precursor protein imported into mitochondria. A means to quantitate translocation contact sites. J Cell Biol, 109(4 Pt 1):1421-1428.

Rassow, J., Harmey, M.A., Müller, H.A., Neupert, W. and Tropschug, M. (1990). Nucleotide sequence of a full-length cDNA coding for the mitochondrial precursor protein of the beta-subunit of F1-ATPase from Neurospora crassa. Nucleic Acids Res, 18(16):4922.

Rehling, P., Brandner, K. and Pfanner, N. (2004). Mitochondrial import and the twinpore translocase. Nat Rev Mol Cell Biol, 5(7):519-530.

Rehling, P., Model, K., Brandner, K., Kovermann, P., Sickmann, A., Meyer, H.E., Kühlbrandt, W. et al. (2003). Protein insertion into the mitochondrial inner membrane by a twin-pore translocase. Science, 299(5613):1747-1751.

Rehling, P., Wiedemann, N., Pfanner, N. and Truscott, K.N. (2001). The mitochondrial import machinery for preproteins. Crit. Rev. Biochem. Mol. Biol., 36(3):291-336.

Reinhold, R., Krüger, V., Meinecke, M., Schulz, C., Schmidt, B., Grunau, S.D., Guiard, B. et al. (2012). The Channel-Forming Sym1 Protein Is Transported by the TIM23 Complex in a Presequence-Independent Manner. Mol Cell Biol, 32(24):5009-5021.

Rios, P., Ben-Zvi, A., Slutsky, O., Azem, A. and Goloubinoff, P. (2006). Hsp70 chaperones accelerate protein translocation and the unfolding of stable protein aggregates by entropic pulling. Proc Natl Acad Sci USA, 103(16):6166-6171.

Roise, D., Horvath, S., Tomich, J., Richards, J. and Schatz, G. (1986). A chemically synthesized pre-sequence of an imported mitochondrial protein can form an amphiphilic helix and perturb natural and artificial phospholipid bilayers. EMBO J, 5(6):1327-1334.

Roise, D., Theiler, F., Horvath, S., Tomich, J., Richards, J., Allison, D. and Schatz, G. (1988). Amphiphilicity is essential for mitochondrial presequence function. EMBO J, $7(3): 649-653$.

Rojo, E.E., Guiard, B., Neupert, W. and Stuart, R.A. (1998). Sorting of D-lactate dehydrogenase to the inner membrane of mitochondria. Analysis of topogenic signal and energetic requirements. J Biol Chem, 273(14):8040-8047. 
Rowland, A.A. and Voeltz, G.K. (2012). Endoplasmic reticulum-mitochondria contacts: function of the junction. Nat Rev Mol Cell Biol, 13(10):607-615.

Rowley, N., Prip-Buus, C., Westermann, B., Brown, C., Schwarz, E., Barrell, B. and Neupert, W. (1994). Mdj1p, a novel chaperone of the DnaJ family, is involved in mitochondrial biogenesis and protein folding. Cell, 77(2):249-259.

Russell, R., Jordan, R. and McMacken, R. (1998). Kinetic characterization of the ATPase cycle of the DnaK molecular chaperone. Biochemistry, 37(2):596-607.

Ryan, M.T., Müller, H. and Pfanner, N. (1999). Functional staging of ADP/ATP carrier translocation across the outer mitochondrial membrane. J Biol Chem, 274(29):2061920627.

Ryan, M.T., Voos, W. and Pfanner, N. (2001). Assaying protein import into mitochondria. Methods Cell Biol, 65:189-215.

Saitoh, T., Igura, M., Obita, T., Ose, T., Kojima, R., Maenaka, K., Endo, T. et al. (2007). Tom20 recognizes mitochondrial presequences through dynamic equilibrium among multiple bound states. EMBO J, 26(22):4777-4787.

Sambrook, J.J. and Russell, D.D.W. (2001). Molecular cloning. A laboratory manual. CSHL Press, 3rd edn.

Sanjuán Szklarz, L.K., Guiard, B., Rissler, M., Wiedemann, N., Kozjak, V., van der Laan, M., Lohaus, C. et al. (2005). Inactivation of the mitochondrial Heat Shock Protein Zim17 Leads to Aggregation of Matrix Hsp70s Followed by Pleiotropic Effects on Morphology and Protein Biogenesis. J Mol Biol, 351(1):206-218.

Saracco, S.A. and Fox, T.D. (2002). Cox18p is required for export of the mitochondrially encoded Saccharomyces cerevisiae Cox2p C-tail and interacts with Pnt1p and Mss2p in the inner membrane. Mol Biol Cell, 13(4):1122-1131.

Saraste, M. (1999). Oxidative phosphorylation at the fin de siècle. Science, 283(5407):1488-1493.

Schägger, H. (2006). Tricine-SDS-PAGE. Nature Protocols, 1(1):16-22.

Schägger, H. and von Jagow, G. (1991). Blue native electrophoresis for isolation of membrane protein complexes in enzymatically active form. Anal Biochem, 199(2):223-231.

Schatz, G. and Butow, R.A. (1983). How are proteins imported into mitochondria? Cell, $32(2): 316-318$. 
Schilke, B. and Craig, E.A. (2011). Reevaluation of the role of the Pam18:Pam16 interaction in translocation of proteins by the mitochondrial Hsp70-based import motor. Mol Biol Cell, 22(24):4740-4749.

Schilke, B.A., Hayashi, M. and Craig, E.A. (2012). Genetic analysis of complex interactions among components of the mitochondrial import motor and translocon in Saccharomyces cerevisiae. Genetics, 190(4):1341-1353.

Schiller, D. (2009). Pam17 and Tim44 act sequentially in protein import into the mitochondrial matrix. Int J Biochem Cell Biol, 41(11):2343-2349.

Schiller, D., Cheng, Y., Liu, Q., Walter, W. and Craig, E.A. (2008). Residues of Tim44 involved in both association with the translocon of the inner mitochondrial membrane and regulation of mitochondrial Hsp70 tethering. Mol Cell Biol, 28(13):4424-4433.

Schlame, M. (2013). Cardiolipin remodeling and the function of tafazzin. Biochim Biophys Acta, 1831(3):582-588.

Schmidt, O., Harbauer, A.B., Rao, S., Eyrich, B., Zahedi, R.P., Stojanovski, D., Schönfisch, B. et al. (2011). Regulation of Mitochondrial Protein Import by Cytosolic Kinases. Cell, 144(2):227-239.

Schmidt, S., Strub, A., Röttgers, K., Zufall, N. and Voos, W. (2001). The two mitochondrial heat shock proteins 70, Ssc1 and Ssq1, compete for the cochaperone Mge1. J Mol Biol, 313(1):13-26.

Schneider, H., Berthold, J., Bauer, M., Dietmeier, K., Guiard, B., Brunner, M. and Neupert, W. (1994). Mitochondrial Hsp70/MIM44 complex facilitates protein import. Nature, 371(6500):768-774.

Schneider, H., Söllner, T., Dietmeier, K., Eckerskorn, C., Lottspeich, F., Trülzsch, B., Neupert, W. et al. (1991). Targeting of the master receptor MOM19 to mitochondria. Science, 254(5038):1659-1662.

Schneider, H., Westermann, B., Neupert, W. and Brunner, M. (1996). The nucleotide exchange factor MGE exerts a key function in the ATP-dependent cycle of mt-Hsp70Tim44 interaction driving mitochondrial protein import. EMBO J, 15(21):5796-5803.

Schulz, C., Lytovchenko, O., Melin, J., Chacinska, A., Guiard, B., Neumann, P., Ficner, R. et al. (2011). Tim50's presequence receptor domain is essential for signal driven transport across the TIM23 complex. J Cell Biol, 195(4):643-656.

Shariff, K., Ghosal, S. and Matouschek, A. (2004). The force exerted by the membrane potential during protein import into the mitochondrial matrix. Biophysj, 86(6):36473652. 
Shiflett, A.M. and Johnson, P.J. (2010). Mitochondrion-related organelles in eukaryotic protists. Annu. Rev. Microbiol., 64:409-429.

Shiota, T., Mabuchi, H., Tanaka-Yamano, S., Yamano, K. and Endo, T. (2011). In vivo protein-interaction mapping of a mitochondrial translocator protein Tom22 at work. Proc Natl Acad Sci USA, 108(37):15179-15183.

Sichting, M., Mokranjac, D., Azem, A., Neupert, W. and Hell, K. (2005). Maintenance of structure and function of mitochondrial Hsp70 chaperones requires the chaperone Hep1. EMBO J, 24(5):1046-1056.

Sickmann, A., Reinders, J., Wagner, Y., Joppich, C., Zahedi, R., Meyer, H.E., Schönfisch, B. et al. (2003). The proteome of Saccharomyces cerevisiae mitochondria. Proc Natl Acad Sci USA, 100(23):13207-13212.

Sideris, D.P., Petrakis, N., Katrakili, N., Mikropoulou, D., Gallo, A., Ciofi-Baffoni, S., Banci, L. et al. (2009). A novel intermembrane space-targeting signal docks cysteines onto Mia40 during mitochondrial oxidative folding. J Cell Biol, 187(7):1007-1022.

Sikor, M., Mapa, K., von Voithenberg, L.V., Mokranjac, D. and Lamb, D.C. (2013). Realtime observation of the conformational dynamics of mitochondrial Hsp70 by spFRET. EMBO J, 32(11):1639-1649.

Sikorski, R.S. and Hieter, P. (1989). A system of shuttle vectors and yeast host strains designed for efficient manipulation of DNA in Saccharomyces cerevisiae. Genetics, 122(1):19-27.

Simon, S., Peskin, C. and Oster, G. (1992). What drives the translocation of proteins? Proc Natl Acad Sci USA, 89(9):3770-3774.

Sirrenberg, C., Endres, M., Fölsch, H., Stuart, R.A., Neupert, W. and Brunner, M. (1998). Carrier protein import into mitochondria mediated by the intermembrane proteins Tim10/Mrs11 and Tim12/Mrs5. Nature, 391(6670):912-915.

Smits, P., Smeitink, J. and van den Heuvel, L. (2010). Mitochondrial translation and beyond: processes implicated in combined oxidative phosphorylation deficiencies. $J$. Biomed. Biotechnol., 2010:737385.

Stojanovski, D., Milenkovic, D., Müller, J.M., Gabriel, K., Schulze-Specking, A., Baker, M.J., Ryan, M.T. et al. (2008). Mitochondrial protein import: precursor oxidation in a ternary complex with disulfide carrier and sulfhydryl oxidase. J Cell Biol, 183(2):195202.

Stojanovski, D., Pfanner, N. and Wiedemann, N. (2007). Import of proteins into mitochondria. Methods Cell Biol, 80:783-806. 
Strauss, M., Hofhaus, G., Schröder, R.R. and Kühlbrandt, W. (2008). Dimer ribbons of ATP synthase shape the inner mitochondrial membrane. EMBO J, 27(7):1154-1160.

Stuart, R. (2002). Insertion of proteins into the inner membrane of mitochondria: the role of the Oxa1 complex. Biochim Biophys Acta, 1592(1):79-87.

Stuart, R.A., Gruhler, A., van der Klei, I., Guiard, B., Koll, H. and Neupert, W. (1994). The requirement of matrix ATP for the import of precursor proteins into the mitochondrial matrix and intermembrane space. Eur J Biochem, 220(1):9-18.

Swain, J., Dinler, G., Sivendran, R., Montgomery, D., Stotz, M. and Gierasch, L.M. (2007). Hsp70 chaperone ligands control domain association via an allosteric mechanism mediated by the interdomain linker. Mol Cell, 26(1):27-39.

Sylvestre, J., Vialette, S., Corral Debrinski, M. and Jacq, C. (2003). Long mRNAs coding for yeast mitochondrial proteins of prokaryotic origin preferentially localize to the vicinity of mitochondria. Genome Biol, 4(7):R44.

Szklarczyk, R. and Huynen, M.A. (2010). Mosaic origin of the mitochondrial proteome. Proteomics, 10(22):4012-4024.

Szyrach, G., Ott, M., Bonnefoy, N., Neupert, W. and Herrmann, J.M. (2003). Ribosome binding to the Oxa1 complex facilitates co-translational protein insertion in mitochondria. EMBO J, 22(24):6448-6457.

Tamura, Y., Harada, Y., Shiota, T., Yamano, K., Watanabe, K., Yokota, M., Yamamoto, H. et al. (2009). Tim23-Tim50 pair coordinates functions of translocators and motor proteins in mitochondrial protein import. J Cell Biol, 184(1):129-141.

Tamura, Y., Harada, Y., Yamano, K., Watanabe, K., Ishikawa, D., Ohshima, C., ichi Nishikawa, S. et al. (2006). Identification of Tam41 maintaining integrity of the TIM23 protein translocator complex in mitochondria. J Cell Biol, 174(5):631-637.

Teixeira, P.F. and Glaser, E. (2013). Processing peptidases in mitochondria and chloroplasts. BBA - Molecular Cell Research, 1833(2):360-370.

Thornton, N., Stroud, D.A., Milenkovic, D., Guiard, B., Pfanner, N. and Becker, T. (2010). Two modular forms of the mitochondrial sorting and assembly machinery are involved in biogenesis of alpha-helical outer membrane proteins. J Mol Biol, 396(3):540549 .

Treco, D.A. and Winston, F. (2001). Growth and Manipulation of Yeast. John Wiley \& Sons, Inc., Hoboken, NJ, USA. 
Truscott, K., Kovermann, P., Geissler, A., Merlin, A., Meijer, M., Driessen, A., Rassow, J. et al. (2001). A presequence- and voltage-sensitive channel of the mitochondrial preprotein translocase formed by Tim23. Nat Struct Biol, 8(12):1074-1082.

Truscott, K.N., Voos, W., Frazier, A.E., Lind, M., Li, Y., Geissler, A., Dudek, J. et al. (2003). A J-protein is an essential subunit of the presequence translocase-associated protein import motor of mitochondria. J Cell Biol, 163(4):707-713.

Ungermann, C., Neupert, W. and Cyr, D. (1994). The role of Hsp70 in conferring unidirectionality on protein translocation into mitochondria. Science, 266(5188):1250-1253.

Verner, K. (1993). Co-translational protein import into mitochondria: an alternative view. Trends in Biochem Sci, 18(10):366-371.

Vestweber, D. and Schatz, G. (1988). Mitochondria can import artificial precursor proteins containing a branched polypeptide chain or a carboxy-terminal stilbene disulfonate. $J$ Cell Biol, 107(6 Pt 1):2045-2049.

Vogel, F., Neupert, W. and Reichert, A.S. (2006). Dynamic subcompartmentalization of the mitochondrial inner membrane. J Cell Biol, 175(2):237-247.

Vögtle, F.N., Burkhart, J.M., Rao, S., Gerbeth, C., Hinrichs, J., Martinou, J.C., Chacinska, A. et al. (2012). Intermembrane space proteome of yeast mitochondria. Mol Cell Proteomics, 11(12):1840-1852.

Vögtle, F.N., Prinz, C., Kellermann, J., Lottspeich, F., Pfanner, N. and Meisinger, C. (2011). Mitochondrial protein turnover: role of the precursor intermediate peptidase Oct1 in protein stabilization. Mol Biol Cell, 22(13):2135-2143.

Vögtle, F.N., Zahedi, R., Becker, D., Voos, W., Sickmann, A., Pfanner, N. and Meisinger, C. (2009). Global analysis of the mitochondrial N-proteome identifies a processing peptidase critical for protein stability. Cell, 139(2):428-439.

Voisine, C., Craig, E.A., Zufall, N., von Ahsen, O., Pfanner, N. and Voos, W. (1999). The protein import motor of mitochondria: unfolding and trapping of preproteins are distinct and separable functions of matrix Hsp70. Cell, 97(5):565-574.

Von Der Malsburg, K., Müller, J.M., Bohnert, M., Oeljeklaus, S., Kwiatkowska, P., Becker, T., Loniewska-Lwowska, A. et al. (2011). Dual role of mitofilin in mitochondrial membrane organization and protein biogenesis. Developmental Cell, 21(4):694-707.

Voos, W. (2013). Chaperone-protease networks in mitochondrial protein homeostasis. BBA - Molecular Cell Research, 1833(2):388-399. 
Voos, W., von Ahsen, O., Müller, H., Guiard, B., Rassow, J. and Pfanner, N. (1996). Differential requirement for the mitochondrial Hsp70-Tim44 complex in unfolding and translocation of preproteins. EMBO J, 15(11):2668-2677.

Voss, C., Lahiri, S., Young, B.P., Loewen, C.J. and Prinz, W.A. (2012). ER-shaping proteins facilitate lipid exchange between the ER and mitochondria in S. cerevisiae. $J$ Cell Sci, 125(20):4791-4799.

Wagener, N., Ackermann, M., Funes, S. and Neupert, W. (2011). A pathway of protein translocation in mitochondria mediated by the AAA-ATPase Bcs1. Mol Cell, 44(2):191202.

Wagener, N. and Neupert, W. (2012). Bcs1, a AAA protein of the mitochondria with a role in the biogenesis of the respiratory chain. J Struct Biol, 179(2):121-125.

Wagner, K., Gebert, N., Guiard, B., Brandner, K., Truscott, K.N., Wiedemann, N., Pfanner, N. et al. (2008). The assembly pathway of the mitochondrial carrier translocase involves four preprotein translocases. Mol Cell Biol, 28(13):4251-4260.

Wallace, D.C. (2007). Why do we still have a maternally inherited mitochondrial DNA? Insights from evolutionary medicine. Annu Rev Biochem, 76:781-821.

Walther, D.M. and Rapaport, D. (2009). Biogenesis of mitochondrial outer membrane proteins. BBA - Molecular Cell Research, 1793(1):42-51.

Webb, C.T., Gorman, M.A., Lazarou, M., Ryan, M.T. and Gulbis, J.M. (2006). Crystal structure of the mitochondrial chaperone TIM9.10 reveals a six-bladed alpha-propeller. Mol Cell, 21(1):123-133.

Weis, B.L., Schleiff, E. and Zerges, W. (2013). Protein targeting to subcellular organelles via mRNA localization. BBA - Molecular Cell Research, 1833(2):260-273.

Weiss, C., Oppliger, W., Vergères, G., Demel, R., Jenö, P., Horst, M., de Kruijff, B. et al. (1999). Domain structure and lipid interaction of recombinant yeast Tim44. Proc Natl Acad Sci USA, 96(16):8890-8894.

Westermann, B. (2010). Mitochondrial fusion and fission in cell life and death. Nat Rev Mol Cell Biol, 11(12):872-884.

Westermann, B. and Neupert, W. (1997). Mdj2p, a novel DnaJ homolog in the mitochondrial inner membrane of the yeast Saccharomyces cerevisiae. J Mol Biol, 272(4):477483.

Wiedemann, N., van der Laan, M., Hutu, D.P., Rehling, P. and Pfanner, N. (2007). Sorting switch of mitochondrial presequence translocase involves coupling of motor module to respiratory chain. J Cell Biol, 179(6):1115-1122. 
Wiedemann, N., Pfanner, N. and Ryan, M.T. (2001). The three modules of ADP/ATP carrier cooperate in receptor recruitment and translocation into mitochondria. EMBO $J, 20(5): 951-960$.

Wiedemann, N., Truscott, K.N., Pfannschmidt, S., Guiard, B., Meisinger, C. and Pfanner, N. (2004). Biogenesis of the protein import channel Tom40 of the mitochondrial outer membrane: intermembrane space components are involved in an early stage of the assembly pathway. J Biol Chem, 279(18):18188-18194.

van Wilpe, S., Ryan, M.T., Hill, K., Maarse, A.C., Meisinger, C., Brix, J., Dekker, P.J. et al. (1999). Tom22 is a multifunctional organizer of the mitochondrial preprotein translocase. Nature, 401(6752):485-489.

Wittelsberger, A., Thomas, B., Mierke, D. and Rosenblatt, M. (2006). Methionine acts as a "magnet" in photoaffinity crosslinking experiments. FEBS Lett, 580(7):1872-1876.

Yamamoto, H., Esaki, M., Kanamori, T., Tamura, Y., ichi Nishikawa, S. and Endo, T. (2002). Tim50 is a subunit of the TIM23 complex that links protein translocation across the outer and inner mitochondrial membranes. Cell, 111(4):519-528.

Yamamoto, H., Fukui, K., Takahashi, H., Kitamura, S., Shiota, T., Terao, K., Uchida, M. et al. (2009). Roles of Tom70 in import of presequence-containing mitochondrial proteins. J Biol Chem, 284(46):31635-31646.

Yamamoto, H., Itoh, N., Kawano, S., Yatsukawa, Y.I., Momose, T., Makio, T., Matsunaga, M. et al. (2011). Dual role of the receptor Tom20 in specificity and efficiency of protein import into mitochondria. Proc Natl Acad Sci USA, 108(1):91-96.

Yamamoto, H., Momose, T., Yatsukawa, Y.I., Ohshima, C., Ishikawa, D., Sato, T., Tamura, Y. et al. (2005). Identification of a novel member of yeast mitochondrial Hsp70-associated motor and chaperone proteins that facilitates protein translocation across the inner membrane. FEBS Lett, 579(2):507-511.

Yamano, K., Kuroyanagi-Hasegawa, M., Esaki, M., Yokota, M. and Endo, T. (2008a). Step-size analyses of the mitochondrial Hsp70 import motor reveal the Brownian ratchet in operation. J Biol Chem, 283(40):27325-27332.

Yamano, K., Tanaka-Yamano, S. and Endo, T. (2010a). Mdm10 as a dynamic constituent of the TOB/SAM complex directs coordinated assembly of Tom40. EMBO Rep, 11(3):187-193.

Yamano, K., Tanaka-Yamano, S. and Endo, T. (2010b). Tom7 regulates Mdm10mediated assembly of the mitochondrial import channel protein Tom40. J Biol Chem, 285(53):41222-41231. 
Yamano, K., Yatsukawa, Y.I., Esaki, M., Hobbs, A., Jensen, R.E. and Endo, T. (2008b). Tom20 and Tom22 share the common signal recognition pathway in mitochondrial protein import. J Biol Chem, 283(7):3799-3807.

Yano, M., Terada, K. and Mori, M. (2004). Mitochondrial import receptors Tom20 and Tom22 have chaperone-like activity. J Biol Chem, 279(11):10808-10813.

Youle, R.J. and Narendra, D.P. (2011). Mechanisms of mitophagy. Nat Rev Mol Cell Biol, 12(1):9-14.

Zahedi, R.P., Sickmann, A., Boehm, A.M., Winkler, C., Zufall, N., Schönfisch, B., Guiard, B. et al. (2006). Proteomic analysis of the yeast mitochondrial outer membrane reveals accumulation of a subclass of preproteins. Mol Biol Cell, 17(3):1436-1450.

Zhang, Y., Deng, H., Zhang, X. and Li, S.J. (2012a). Interaction of divalent metal ions with human translocase of inner membrane of mitochondria Tim50. Biochem Biophys Res Commun, pp. 1-6.

Zhang, Y., Deng, H., Zhao, Q. and Li, S.J. (2012b). Interaction of presequence with human translocase of the inner membrane of mitochondria Tim50. J Phys Chem B, 116(9):2990-2998.

Zheng, L. (2004). An efficient one-step site-directed and site-saturation mutagenesis protocol. Nucleic Acids Research, 32(14):e115-e115.

Zhu, X., Zhao, X., Burkholder, W., Gragerov, A., Ogata, C., Gottesman, M. and Hendrickson, W. (1996). Structural analysis of substrate binding by the molecular chaperone DnaK. Science, 272(5268):1606-1614.

Zick, M., Rabl, R. and Reichert, A.S. (2009). Cristae formation-linking ultrastructure and function of mitochondria. BBA - Molecular Cell Research, 1793(1):5-19. 


\section{Acknowledgements}

Zuallererst möchte ich mich recht herzlich bei Prof. Dr. Peter Rehling bedanken. Die vier Jahre in seinem Labor waren eine hervorragende Zeit. Ich bin Ihm für das Thema und die ständige Diskussionsbereitschaft, die tolle Betreuung, Forderung und Förderung sehr dankbar.

Desweiteren danke ich auch Prof. Dr. Kai Tittmann und Prof. Dr. Dirk Görlich für die Diskussion meiner Arbeit in den Thesis Committee Sitzungen und der praktischen Unterstützung mit verschiedenen Materialen und Methoden.

Ich bedanke mich auch bei allen aktuellen und ehemaligen Mitarbeitern des Institutes für zelluläre Biochemie für das tolle Arbeitsklima. Ganz besonders möchte ich mich bei Dr. David U. Mick, Dr. Robert Reinhold, Dr. Sven Dennerlein, Dr. Markus Deckers, und Bettina Bareth bedanken. Hervorheben möchte ich auch die tolle Zusammenarbeit der Import Gruppe mit Dr. Oleksander Lytovchenko und Jonathan Melin. Ich möchte mich auch bei den verschiedenen Studenten bedanken, mit denen ich zusammen arbeiten durfte.

Vielen Dank auch an die vielen kleinen und großen Kollaborationen: Dr. Olaf Jahn (MPIem) und Lars van Werven für die Peptidesynthesen und Reinigungen, Dr. Bernhard Schmidt, Olaf Bernhard und Dr. Olaf Jahn für die Massenspektrometrie, Klaus Neifer für die Hilfe bei den verschiedenen Proteinreinigungen und Prof. Dr. Ralf Ficner und Dr. Achim Dickmanns für die kontinuierlichen Kristallisationsversuche.

Für die Unterstützung durch die IMPRS Molecular Biology, die GGNB und den Boehringer Ingelheim Fond bin ich ebenfalls sehr dankbar.

Zuletzt danke ich besonders meiner Familie und Natalia für die langjährige und uneingeschränkte Unterstützung. 